\title{
ANÁlise NÃO-LINEAR DE PÓRTICOS PLANOS DE CONCRETO ARMADO: MODELAGEM NUMÉRICA E AVALIAÇÃO DOS MÉTODOS APROXIMADOS
}

\author{
ROBERTO MÁRCIO DA SILVA
}

Tese apresentada à Escola de Engenharia de São Carlos, da Universidade de São Paulo, como parte dos requisitos para obtenção do Título de Doutor em Engenharia Civil.

ORIENTADORA: Prof ${ }^{a} \mathrm{Dr}^{\mathrm{a}}$ Helena M. C. Carmo Antunes

SÃO CARLOS

1996 
S583a Análise não-linear de pórticos planos de concreto armado: modelagem numérica e avaliação dos métodos aproximados / Roberto Márcio da Silva -São Carlos, 1996.

Tese (Doutorado) -- Escola de Engenharia de São Carlos Universidade de São Paulo, 1996.

Orientadora: Profa. Dra. Helena M.C. Carmo Antunes

1. Estruturas de concreto. 2. Análise não-linear. I. Titulo. 
Aos meus pais,

à minha esposa Ubaldina,

aos meus filhos

Thiago e Leandro,

aos meus irmãos e à tia Elzira 


\section{AGRADECIMENTOS}

À Prof ${ }^{a} r^{a}$ Helena M. C. Carmo Antunes, pelo apoio, pela orientação que permitiram a conclusão deste trabalho.

Ao Prof. Sérgio Persival Baroncini Proença pelo auxílio, pelas sugestões e, sobretudo, pela amizade.

Aos professores e funcionários do Departamento de Estruturas da Escola de Engenharia de São Carlos, da Universidade de São Paulo (EESCUSP).

Ao Departamento de Engenharia de Estruturas da Escola de Engenharia da UFMG que tornou possível a execução do presente trabalho.

Aos colegas do Departamento de Estruturas da EESC, pelo agradável convivio, pela amizade, pelas sugestões e colaborações.

Ao engenheiro pós-graduando Luiz Antonio de Souza pela dedicação e capricho na montagem deste trabalho.

À CAPES, pelo auxílio financeiro. 


\section{SUMÁRIO}

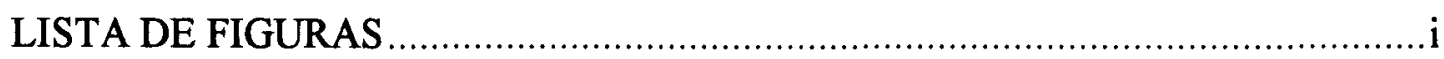

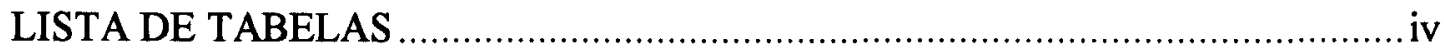

LISTA DE SÍMBOLOS ........................................................................... vii

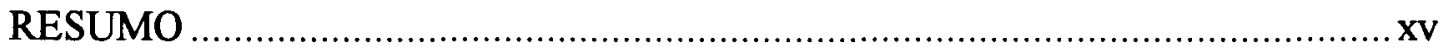

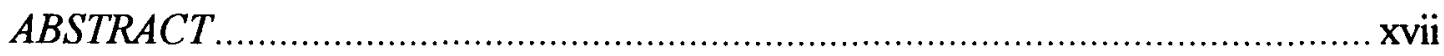

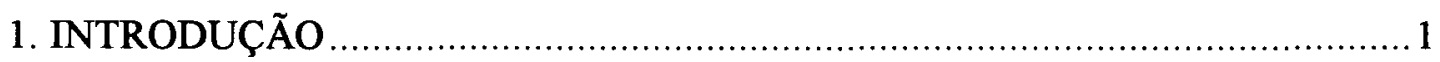

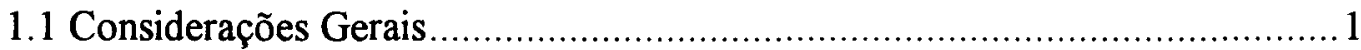

1.2 Modelos de Comportamento dos Materiais ...........................................2

1.2.1 Modelo Elástico................................................................................

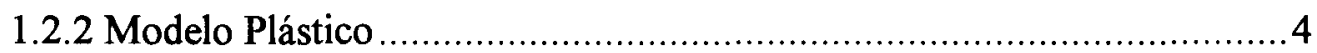

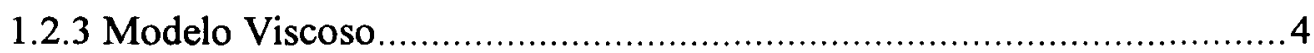

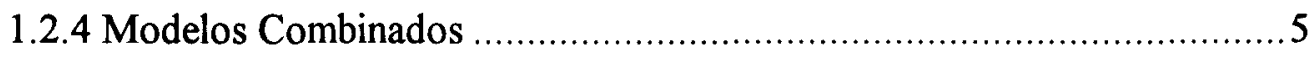

1.3 Interação Entre Forças e Deslocamentos .................................................... 8

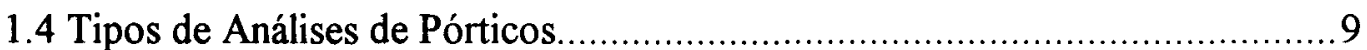

1.5 Considerações Sobre o Comportamento do Concreto Armado ....................12

1.6 Considerações Gerais Sobre os Métodos de Projetos em Concreto Armado 15

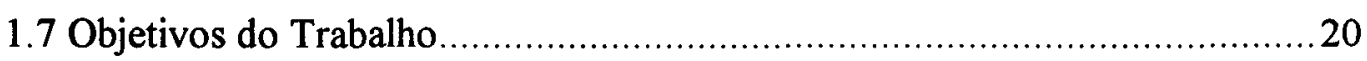

1.8 Descrição Resumida dos Capítulos................................................... 21

2. PRESCRIÇÕES NORMATIVAS PARA ANÁLISE NÃO-LINEAR

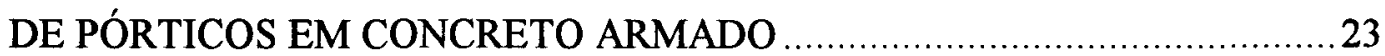

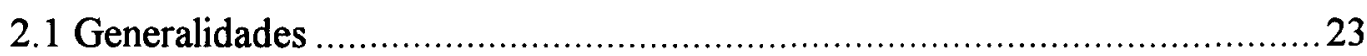

2.2 Métodos de Análise Não-linear ...........................................................23

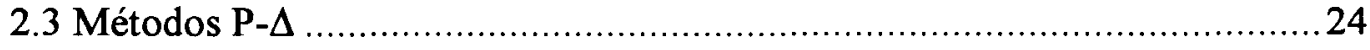

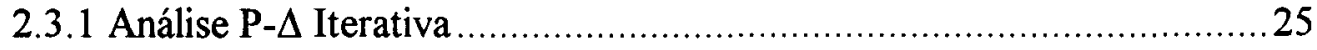

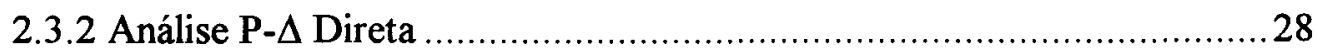

2.3.3. Método P- $\triangle$ Amplificador de Momento ..................................... 33

2.4 Métodos Baseados no Comprimento Efetivo da Coluna ............................37

2.4.1 Comprimento Efetivo de Barras Compridas ................................. 38 
2.4.2 Considerações Sobre os Efeitos da Esbeltez

2.4.3 Método do Amplificador de Momentos............................................. 44

2.4.4 Método do Momento Adicional ...................................................48

2.5 Comentários Finais Sobre os Métodos Simplificados para Análise

Não-linear de Pórticos em Concreto Armado

\section{FORMULAÇÃo CONSISTENTE PARA ANÁLISE NÃO-LINEAR}

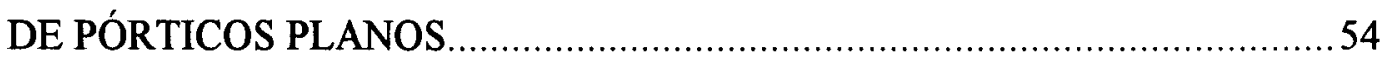

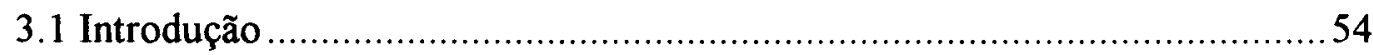

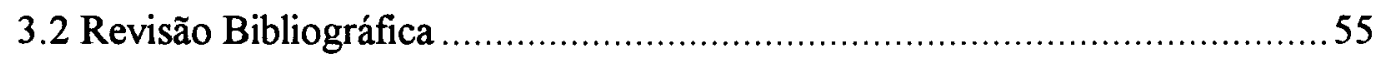

3.3 Considerações Sobre as Formulações para Análise Não-linear via

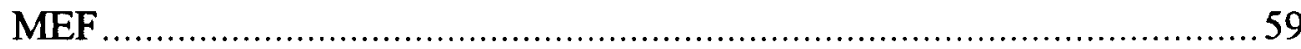

3.4 Considerações Sobre os Sistemas de Coordenadas ...................................61

3.5 Considerações Sobre as Deformações e as Tensões......................................64

3.6 Definição do Modelo Elemental. Graus de Liberdade e

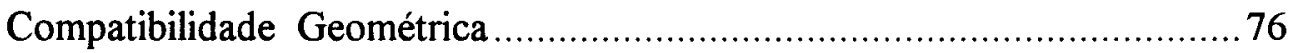

3.7 Teoria Estrutural. Definição dos Campos de Deformações e de

Deslocamentos do Elemento .................................................................... 95

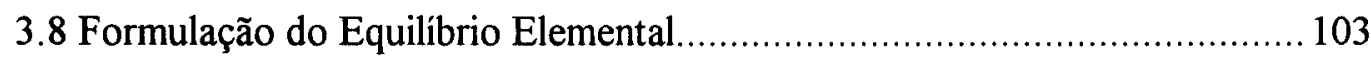

3.9 Matriz de Rigidez Tangente do Elemento ......................................... 106

3.10 Funções de Interpolação dos Deslocamentos..................................... 109

3.11 Aproximações e Simplificações Adotadas............................................... 116

3.11.1- Aproximações de $2^{\mathrm{a}}$ ordem ................................................. 116

3.11.2 - Elemento Prismático em Regime Elástico Linear...................... 125

3.11 .3 - Elemento Prismático em Regime Elasto-plástico ....................... 135

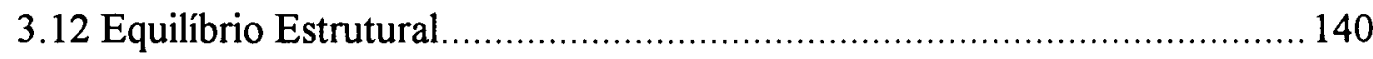

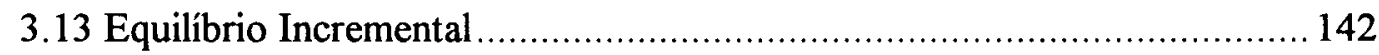

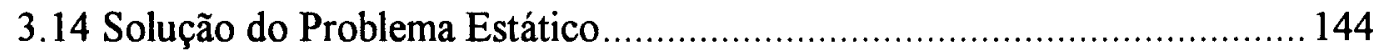

3.14.1 Métodos de Solução de Equações Não-lineares........................... 144

3.14.2 - Método Iterativo de Newton-Raphson....................................... 145

3.14.3 Critérios de Convergência ...................................................... 150 
3.14.4 Análise da Estabilidade .153

3.15 - Exemplos de Estruturas Elásticas Sujeitas a Grandes Deslocamentos ... 154

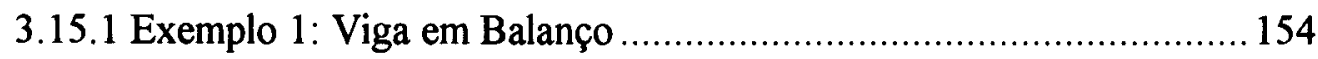

3.15.2 Exemplo 2: Pórtico Losangular sob Carga de Compressão .............. 161

3.15.3 Exemplo 3: Pórtico Losangular sob Carga de Tração ...................... 165

3.15.4 Exemplo 4: Quadro sob Carga de Compressão................................ 170

3.15.5 Exemplo 5: Quadro sob Carga de Tração ......................................... 174

\section{APLICAÇÃO DA FORMULAÇÃO CONSISTENTE À ANÁLISE DE} PÓRTICOS PLANOS DE CONCRETO ARMADO

4.1 Introdução 179

4.2 Revisão Bibliográfica 180

4.3 Elemento Prismático de Concreto Armado 183

4.3.1 Utilização do Processo de Fatias para a Discretização da Seção do

Elemento de Concreto Armado 184

4.3.2 Equações Constitutivas para o Concreto 186

4.3.3 Equações Constitutivas para o Aço 191

4.4 Programa para o Cálculo Automático 193

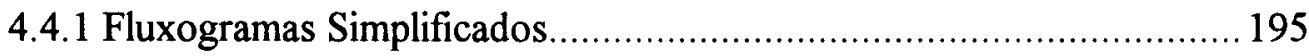

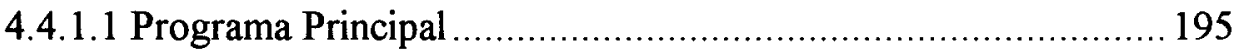

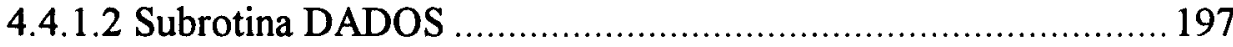

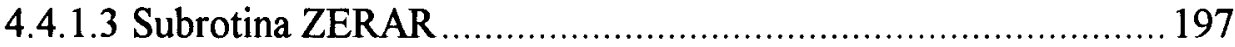

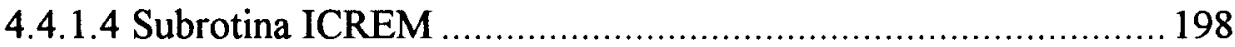

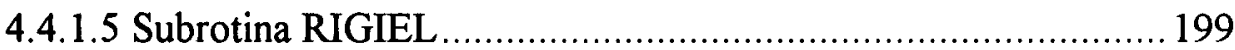

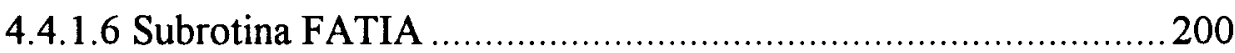

4.4.1.7 Subrotina MONRIG ......................................................... 202

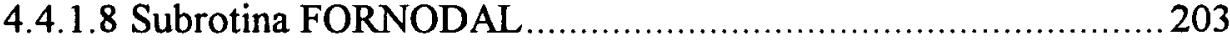

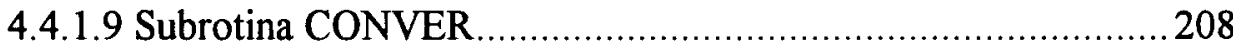

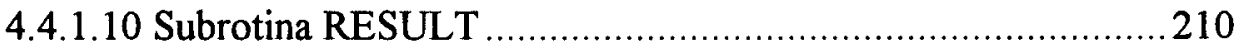

4.5 Exemplos de concreto armado ........................................................... 212

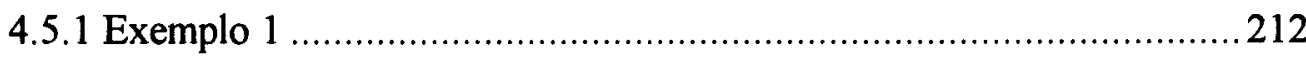




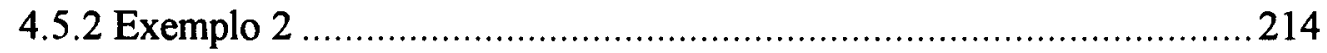

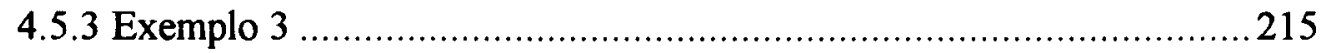

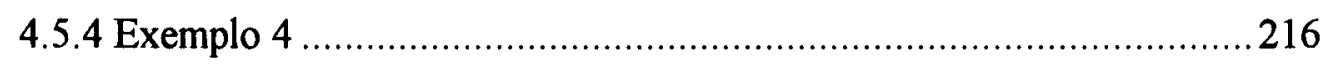

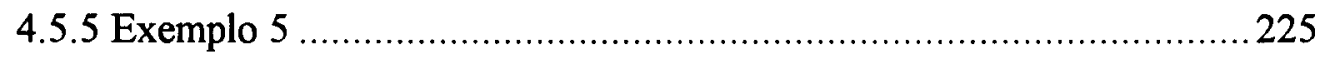

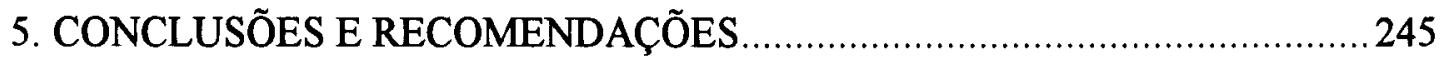

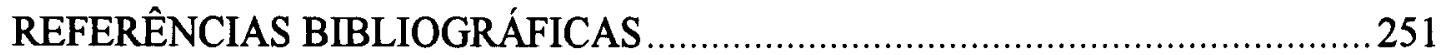




\section{LISTA DE FIGURAS}

FIGURA 1.1 - Modelos materiais idealizados .............................................. 4

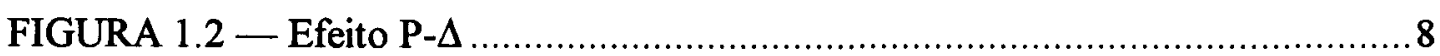

FIGURA 1.3 - Relações carga-deslocamento para pórticos ................................ 9

FIGURA 1.4 - Redistribuição de momentos provocada pela fissuração numa viga hiperestática.............................................................. 14

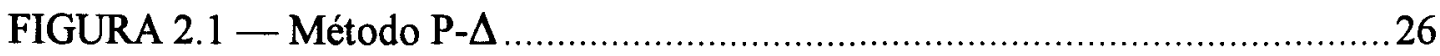

FIGURA 2.2 - Cálculo das forças laterais equivalentes ao efeito P- $\Delta$.................. 27

FIGURA 2.3 - Forças na coluna deformada .................................................... 34

FIGURA 2.4 - Passos de um procedimento simplificado de projeto ...................... 38

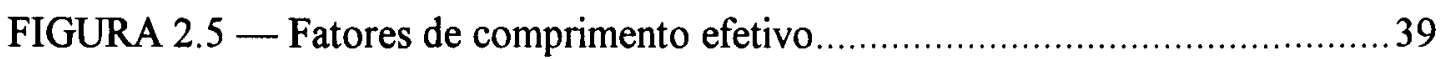

FIGURA 2.6 - Interação de colunas de mesma inércia de um pórtico bi-rotulado .. 46

FIGURA 2.7 - Interação de colunas de inércia diferente um pórtico bi-rotulado .... 47

FIGURA 2.8 - Excentricidades iguais nas extremidades ...................................

FIGURA 2.9 - Excentricidades diferentes nas extremidades .............................50

FIGURA 3.1 - Coordenadas-deslocamento de um elemento de pórtico plano nos sistemas cartesiano e corrotacional ........................................62

FIGURA 3.2 - Configurações de uma Fibra de Material ......................................64

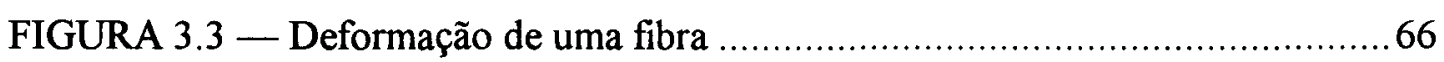

FIGURA 3.4 - Modelo Elemental. Sistemas de Coordenadas. ........................... 77

FIGURA 3.5 - Elemento Diferencial de Barra Reta ......................................... 96

FIGURA 3.6 - Deslocamentos de pontos da barra........................................ 100

FIGURA 3.7 - Elemento diferencial do eixo da barra nas configurações de referência e deformada ........................................................ 101

FIGURA 3.8 - Elemento de barra de pórtico plano .................................... 109

FIGURA 3.9 - Método iterativo de Newton-Raphson. ................................. 146

FIGURA 3.10 - Método de Newton-Raphson com rigidez inicial ...................... 148

FIGURA 3.11 — Método de Newton-Raphson modificado .............................. 149

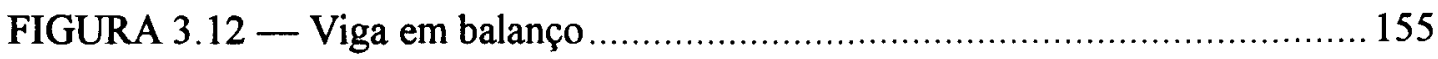


FIGURA 3.13 - Curva carga paramétrica $\mathrm{x}$ deslocamento adimensional $\mathrm{u} / \ell$...... 159

FIGURA 3.14 - Curva carga paramétrica $x$ deslocamento adimensional $v / \ell$...... 154

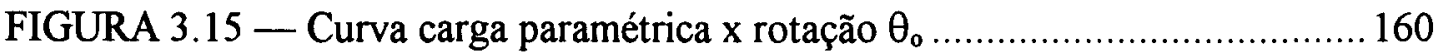

FIGURA 3.16 - Pórtico Losangular sob carga de compressão............................ 162

FIGURA 3.17 - Curva carga paramétrica $\mathrm{x}$ deslocamento adimensional $\mathrm{u} / \ell$....... 164

FIGURA 3.18 - Curva carga paramétrica $\mathrm{x}$ deslocamento adimensional $\mathrm{v} / \ell \ldots \ldots .165$

FIGURA 3.19 - Pórtico Losangular sob carga de tração ................................... 166

FIGURA 3.20 - Curva carga paramétrica $\mathrm{x}$ deslocamento adimensional $\mathrm{u} / \ell$....... 168

FIGURA 3.21 - Curva carga paramétrica $\mathrm{x}$ deslocamento adimensional $\mathrm{v} / \ell$...... 169

FIGURA 3.22 - Quadro sob carga de compressão ........................................... 170

FIGURA 3.23 - Curva carga paramétrica $\mathrm{x}$ deslocamento adimensional $\mathrm{u} / \ell \ldots \ldots .172$

FIGURA 3.24 - Curva carga paramétrica $x$ deslocamento adimensional $\mathrm{v} / \ell \ldots \ldots .173$

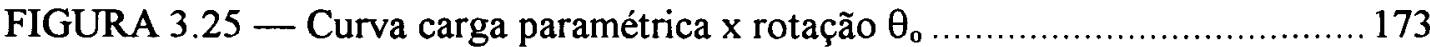

FIGURA 3.26 - Quadro sob carga de tração ................................................ 174

FIGURA 3.27 - Curva carga paramétrica $\mathrm{x}$ deslocamento adimensional $\mathrm{u} / \ell \ldots \ldots .176$

FIGURA 3.28 - Curva carga paramétrica $\mathrm{x}$ deslocamento adimensional $\mathrm{v} / \ell \ldots \ldots .177$

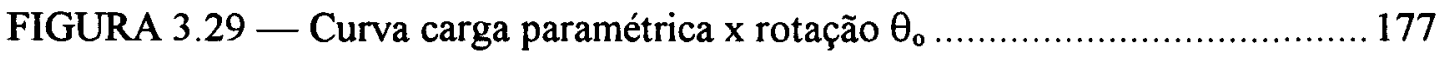

FIGURA 4.1 - Seção tranversal dividida em fatias ......................................... 184

FIGURA 4.2 - Relação tensão-deformação para o concreto em compressão

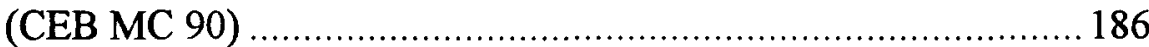

FIGURA 4.3 - Relação tensão-deformação para o concreto em compressão (NBR-6118) 188

FIGURA 4.4 - Relação tensão-deformação para o concreto em tração

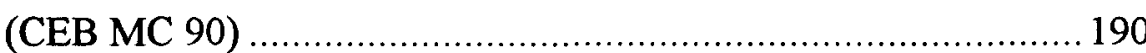

FIGURA 4.5 - Relação tensão-deformação para aço classe A (NBR-6118) ......... 192

FIGURA 4.6 - Relação tensão-deformação para aço classe B (NBR-61 18) ......... 193

FIGURA 4.7 - Sistema local de coordenadas ................................................ 194

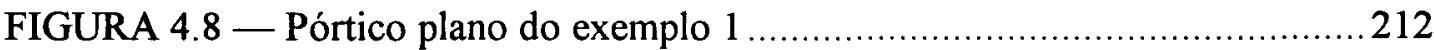

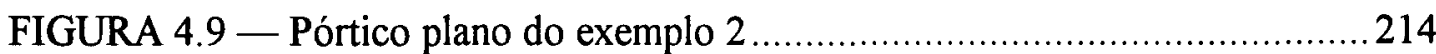

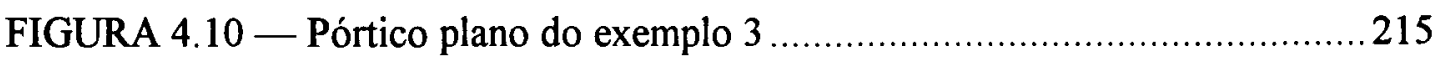

FIGURA 4.11 — Pórtico plano do exemplo 4 ................................................. 217 
FIGURA 4.12 - Numeração das seções do pórtico do exemplo 4 221

FIGURA 4.13 - Estrutura do edifício do exemplo 5 226

FIGURA 4.14 - Pórtico plano com carregamento 1 227

FIGURA 4.15 - Pórtico plano com carregamento 2 228

FIGURA 4.16 - Armadura do pórtico plano do Exemplo 5 229

FIGURA 4.17 - Numeração das seções. 231

FIGURA 4.18 - Curva carga $\mathrm{x}$ deslocamento para o carregamento 1 . 233

FIGURA 4.19 - Curva carga $x$ deslocamento para o carregamento 2 234

FIGURA 4.20 - Curva carga $x$ deslocamento para o carregamento 1 com recalque 236

FIGURA 4.21 - Curva carga x deslocamento para o carregamento 2 com recalque 


\section{LISTA DE TABELAS}

Tabela 3.1 - Valores dos deslocamentos adimensionais $\mathrm{u} / \ell$ na extremidade livre da viga em balanço da Fig. 3.12 .................................................. 156

Tabela 3.2 - Valores dos deslocamentos adimensionais $\mathrm{v} / \ell$ na extremidade livre da viga em balanço da Fig. 3.12

Tabela 3.3 - Valores das rotações $\theta_{0}$ na extremidade livre da viga em balanço da Fig. 3.12

Tabela 3.4 - Valores dos deslocamentos $(u / \ell),(v / \ell)$ e rotações $\theta_{\mathrm{o}}$ na extremidade livre da viga com 10 incrementos de carga

Tabela 3.5 - Valores dos deslocamentos adimensionais $\mathrm{u} / \ell$ do nó $\mathrm{B}$ do pórtico da Fig. 3.16

Tabela 3.6 - Valores dos deslocamentos adimensionais $\mathrm{v} / \ell$ do nó A do pórtico da Fig. 3.16

Tabela 3.7 - Valores dos deslocamentos adimensionais $\mathrm{u} / \ell$ do nó $\mathrm{B}$ do pórtico da Fig. 3.19

Tabela 3.8 - Valores dos deslocamentos adimensionais v/ $\ell$ do nó A do pórtico da Fig. 3.19.

Tabela 3.9 - Valores dos deslocamentos adimensionais $\mathrm{u} / \ell$ do nó $\mathrm{C}$ do quadro da Fig. 3.22.

Tabela 3.10 - Valores dos deslocamentos adimensionais v/ $\ell$ do nó A do quadro da Fig. 3.22

Tabela 3.11 - Valores das rotações $\theta_{0}$ do nó B do quadro da Fig. 3.22

Tabela 3.12 - Valores dos deslocamentos adimensionais $\mathrm{u} / \ell$ do nó $\mathrm{C}$ do quadro da Fig. 3.26.

Tabela 3.13 - Valores dos deslocamentos adimensionais v/ $\ell$ do nó A do quadro da Fig. 3.26

Tabela 3.14 - Valores das rotações $\theta_{0}$ do nó B do quadro da Fig. 3.26 176

Tabela 4.1 - Resultados da análise do pórtico do exemplo 1 213

Tabela 4.2 - Resultados da análise do pórtico do exemplo 2 214 
Tabela 4.3 - Influência da discretização longitudinal das barras. 216

Tabela 4.4 - Deslocamentos laterais do pórtico do exemplo $4(\mathrm{em} \mathrm{cm})$................. 218

Tabela 4.5 - Momentos fletores no pórtico do exemplo 4

Tabela 4.6 - Deslocamentos laterais do pórtico do exemplo 4, via método P- $\Delta$ $(\mathrm{em} \mathrm{cm})$

Tabela 4.7 - Momentos fletores no pórtico do exemplo 4 via método P- $\Delta$ (em KN.m)

Tabela 4.8 - Momentos fletores no pórtico do exemplo 4 224

Tabela 4.9 - Deslocamentos laterais do pórtico do exemplo $5(\mathrm{em} \mathrm{cm})$ 230

Tabela 4.10 - Momentos fletores na viga do $1^{0}$ pavimento do pórtico do exemplo 5

Tabela 4.11 - Deslocamentos laterais do topo do pórtico do exemplo 5 para o carregamento 1

Tabela 4.12 - Deslocamentos laterais do topo do pórtico do exemplo 5 para o carregamento 2

Tabela 4.13 - Deslocamentos laterais do topo do pórtico do exemplo 5 para o carregamento 1 , com recalque

Tabela 4.14 - Deslocamentos laterais do topo do pórtico do exemplo 5 para o carregamento 2 , com recalque

Tabela 4.15 - Deslocamentos laterais do pórtico para o carregamento 1, via método P- $\Delta$

Tabela 4.16 - Deslocamentos laterais do pórtico para o carregamento 2, via método P- $\Delta$

Tabela 4.17 - Momentos fletores no pórtico para o carregamento 1, via método $\mathrm{P}-\Delta$

Tabela 4.18 - Momentos fletores no pórtico para o carregamento 2, via método P- $\Delta$

Tabela 4.19 - Momentos fletores no pórtico para o carregamento 1, via método do amplificador de momentos.

Tabela 4.20 - Momentos fletores no pórtico para o carregamento 2, via método do amplificador de momentos. 
Tabela 4.21 - Deslocamentos laterais do pórtico com carregamento multiplicado por 2 , via método $\mathrm{P}-\Delta$

Tabela 4.22 - Momentos fletores no pórtico com carregamento multiplicado por 2, via método $\mathrm{P}-\Delta$

Tabela 4.23 - Momentos fletores no pórtico com carregamento multiplicado por 2, via método do amplificador de momentos. 


\section{LISTA DE SÍMBOLOS}

a

A

$\mathbf{A}$

$\mathrm{A}_{\mathrm{c}}$

$A_{r}$

$\mathrm{A}_{\mathrm{si}}$

B

b

$\overline{\mathbf{B}}$

$b_{i}$

$\mathrm{C}_{\mathrm{m}}$

D

$\dot{d}$

$\mathrm{d} \alpha$

$\mathrm{D}_{\mathrm{ci}}$

$D^{e}$

$D^{\text {ep }}$

$\mathrm{D}_{\mathrm{m}}$

$\mathrm{D}_{\mathrm{N}}$

$\mathrm{dp}_{\mathrm{i}}$

$d q_{\alpha}$

$\mathrm{ds}_{\mathrm{c}}$

$\mathrm{d} \bar{s}_{\mathrm{c}}$

$\mathrm{D}_{\mathrm{si}}$
- Fator geométrico

- Área da seção transversal

- Matriz de incidência cinemática

- Área da seção transversal geométrica da coluna ou área da seção transversal da barra na configuração deformada ou atual

- Área da seção transversal da barra na configuração de referência

- Área total da armadura na fatia i

- Matriz instantânea de mudança de coordenadas

- Largura da seção transversal

- Forma local da matriz B

- Largura da fatia i

- Fator de correção para levar em conta o fato do momento máximo da coluna não ocorrer nas extremidades

- Módulo de rigidez tangente do material

- Taxa instantânea de deformação

- Deformação angular

- Módulo de rigidez tangente do concreto na fatia $\mathrm{i}$

- Módulo de rigidez tangente elástico

- Módulo de rigidez tangente elasto-plástico

- Família de módulos de rigidez

- Módulo de rigidez nominal

- Taxa de deslocamento na coordenada cartesiana $p_{i}$

- Taxa de deslocamento na coordenada natural $\mathrm{q}_{\alpha}$

- Comprimento de uma fibra genérica situada a uma distância $\mathrm{y}_{\mathrm{r}}$ do eixo do elemento diferencial na configuração deformada

- Comprimento de uma fibra no eixo do elemento diferencial na configuração deformada

- Módulo de rigidez tangente do aço na fatia I 
- Comprimento de uma fibra genérica situada a uma distância $\mathrm{y}_{\mathrm{r}}$ do eixo do elemento diferencial na configuração de referência

$\bar{d} \overline{s_{r}}$

- Comprimento de uma fibra no eixo do elemento diferencial na configuração de referência

$\mathrm{dx}_{\mathrm{r}}$

- Comprimento do elemento diferencial na configuração de referência

E

$\mathrm{e} 0_{1}$

$\mathrm{e}_{2}$

$e_{1}$

$e_{2}$

EA

EAy

$E_{c}$

$\mathrm{E}_{\mathrm{cl}}$

EI

$\mathrm{E}_{\mathrm{s}}$

$E_{v}$

$f_{c}$

$\mathrm{f}_{\mathrm{cd}}$

$f_{\mathrm{ci}}$

$f_{\text {ck }}$

$\mathrm{f}_{\mathrm{ctm}}$

$\mathrm{F}_{\mathrm{j}}$

$\mathrm{f}_{\mathrm{s}}$

$\mathrm{f}_{\mathrm{si}}$

$f_{\mathrm{uk}}$

- Módulo de elasticidade

- Excentricidade equivalente na extremidade 1

- Excentricidade equivalente na extremidade 2

- Excentricidade na extremidade 1

- Excentricidade na extremidade 2 ou excentricidade de $2^{\underline{a}}$ ordem

- Produto de rigidez axial da seção

- Produto do módulo de elasticidade pelo momento estático da seção

- Módulo de elasticidade da coluna ou módulo de elasticidade do concreto

- Módulo de elasticidade secante da origem para o ponto de tensão máxima

- Produto de rigidez à flexão da seção

- Módulo de elasticidade da armadura ou do aço

- Módulo de elasticidade da viga

- Tensão de compressão uniaxial do concreto

- Resistência de cálculo do concreto à compressão

- Tensão no concreto na fatia i

- Resistência característica à compressão do concreto

- Resistência média à tração uniaxial do concreto

- Força lateral fictícia a ser aplicada no andar "j" para simular o efeito P- $\Delta$

- Tensão na armadura

- Tensão na armadura na fatia i

- Resistência característica à tração do concreto 
- Tensão de escoamento da armadura

$\mathrm{f}_{\mathrm{yd}}$

- Resistência de cálculo do aço

$\mathrm{f}_{\mathrm{yk}}$

- Resistência característica do aço

$\overline{\mathbf{G}}_{\alpha}$

- Forma local da matriz $\mathbf{G}_{\alpha}$

$\mathbf{G}_{\alpha}$

- Matriz que contém as derivadas $q_{\alpha, j}$

$\mathbf{G}_{\mathbf{1}}$

- Matriz que contém as derivadas $q_{1, i j}$

$\mathbf{G}_{2}$

- Matriz que contém as derivadas $q_{2, j \mathrm{j}}$

$\mathbf{G}_{3}$

- Matriz que contém as derivadas $q_{3, i j}$

- Força horizontal aplicada na estrutura

- Altura total do pórtico, do andar ou da seção transversal

- Força horizontal total aplicada no andar ou cortante no andar "j"

- Altura do andar " $\mathrm{j}$ "

- Momento de inércia da seção transversal

- Momento de inércia da seção transversal da coluna A

- Momento de inércia da seção transversal da coluna B

IELEM

- Momento de inércia da seção transversal da coluna

- Número do elemento

- Momento de inércia de seção plena da barra

- Momento de inércia da seção transversal da viga

- Curvatura do eixo deformado

- Fator de comprimento efetivo de coluna

- Matriz de rigidez tangente do elemento no sistema de coordenadasdeslocamento cartesiano

- Matriz de rigidez tangente da estrutura

- Comprimento efetivo

- Matriz de rigidez tangente do elemento no sistema de coordenadasdeslocamento natural

- Matriz de rigidez geométrica do elemento

- Deslocamento lateral causado por uma força lateral unitária no andar "j" 


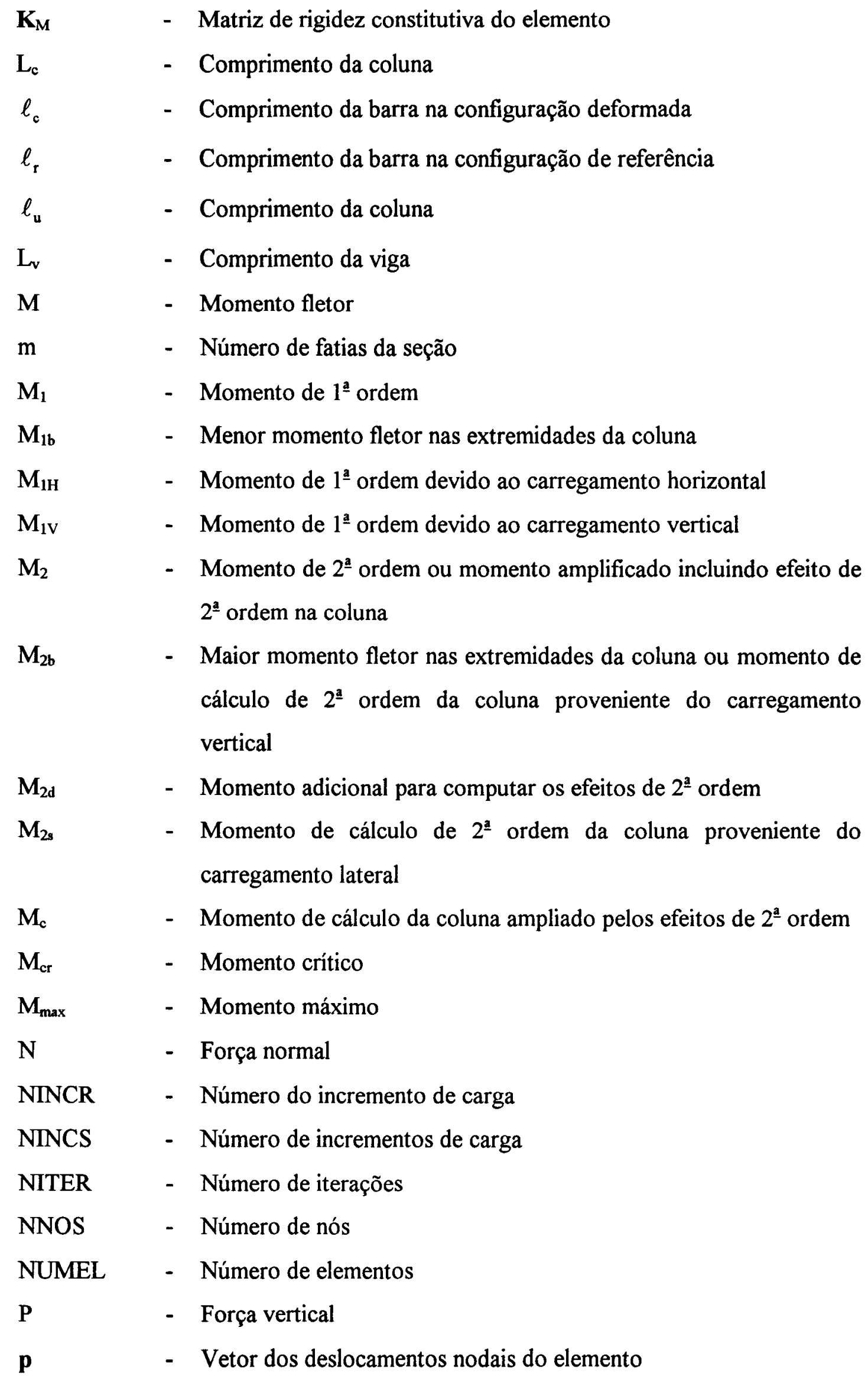




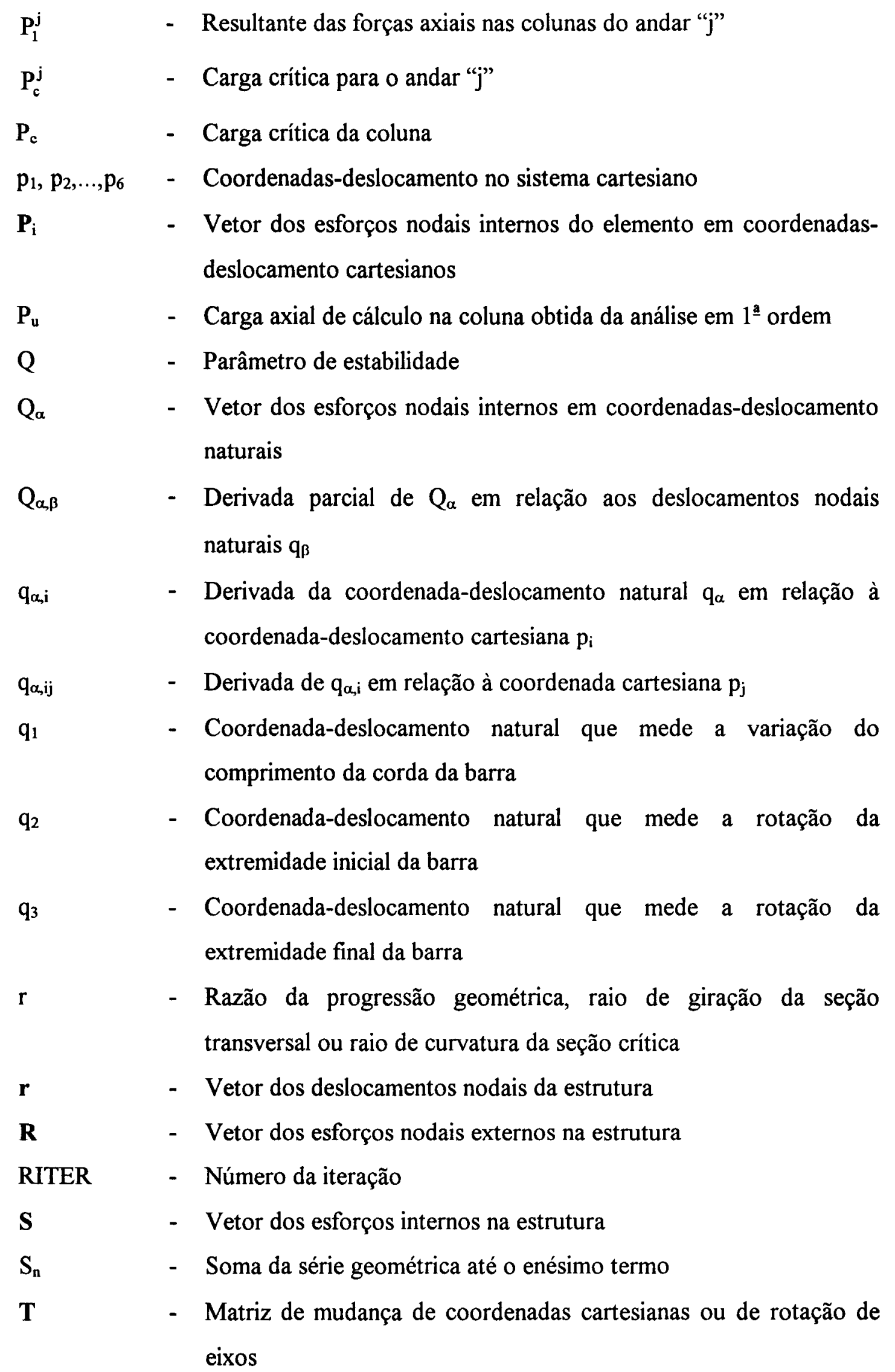


- Altura da fatia i

- Deslocamento axial de um ponto genérico da barra distante $y_{r}$ do eixo

- Deslocamento axial de um ponto sobre o eixo

- Deslocamento axial de um ponto genérico da barra distante $y_{\mathrm{r}}$ do eixo no sistema corrotacional

- Deslocamento axial de um ponto sobre o eixo da barra no sistema corrotacional

- Deslocamento transversal de um ponto genérico da barra distante $y_{r}$ do eixo

- Força cortante

- Deslocamento transversal de um ponto sobre o eixo

- Força cortante adicional no andar “j”

- Volume da barra na configuração deformada ou atual

- Deslocamento transversal de um ponto genérico da barra distante $\mathrm{y}_{\mathrm{r}}$ do eixo no sistema corrotacional

- Deslocamento transversal de um ponto sobre o eixo da barra no sistema corrotacional

- Volume da barra na configuração de referência

- Trabalho da força normal por unidade de volume

- Potência da força normal por unidade de volume

- Trabalho total da força normal

- Coordenadas cartesianas do nó inicial da barra no sistema global

- Coordenadas cartesianas do nó final da barra no sistema global

- Ordenada do CG da seção tranversal

- Ordenada do CG da fatia i

- Ordenada de fibra genérica

- Rotação específica

- Parâmetro de estabilidade ou rotação da seção transversal

- Deslocamento lateral relativo de $1^{\underline{a}}$ ordem do andar " $\mathrm{j}$ " 
$\Delta_{\mathbf{f}}$

$\Delta \mathrm{M}$

$\Delta_{\max }$

$\delta p_{i}$

$\delta \mathrm{s}$

$\delta W_{i}$

$\bar{\varepsilon}$

$\varepsilon$

$\dot{\varepsilon}$

$\dot{\varepsilon}_{\mathrm{n}}$

$\varepsilon_{0}$

$\varepsilon_{-1}$

$\varepsilon_{1}$

$\varepsilon_{-1 / 2}$

$\varepsilon_{1 / 2}$

$\varepsilon_{, \alpha}$

$\varepsilon_{\mathrm{c}}$

$\varepsilon_{\mathrm{cl}}$

$\varepsilon_{\mathrm{D}}$

$\varepsilon_{\mathrm{E}}$

$\varepsilon_{\mathrm{F}}$

$\bar{\varepsilon}_{\mathbf{m}}$

$\varepsilon_{\mathrm{m}}$

- Deslocamento horizontal relativo entre pavimentos

- Deslocamento horizontal relativo de $1^{\underline{a}}$ ordem

- Amplificador do momento de $1^{\underline{a}}$ ordem proveniente do carregamento vertical

- Deformação virtual

- Deslocamento lateral final

- Incremento inicial de momento de $1^{\underline{a}}$ ordem

- Deslocamento lateral máximo

- Deslocamento virtual em coordenadas cartesianas

- Amplificador do momento de $1^{\underline{a}}$ ordem proveniente do carregamento lateral

- Trabalho virtual interno do elemento

- Deformação de uma fibra no eixo

- Deformação de uma fibra genérica distante $Y_{r}$ do eixo

- Taxa de deformação

- Família de taxas de deformação

- Deformação natural ou de Hencky ou deformação correspondente à tensão máxima

- Deformação de Almansi

- Deformação quadrática ou de Green

- Deformação hiperbólica ou de Reiner

- Deformação linear ou de engenharia

- Derivada da deformação $\varepsilon$ em relação a $q_{\alpha}$

- Deformação por compressão do concreto

- Deformação correspondente à tensão máxima de compressão $f_{c m}$

- Tolerância em deslocamentos

- Tolerância em energia interna

- Tolerância em esforços

- Deformação média da fibra sobre o eixo do elemento

- Família de medidas de deformação 


\begin{tabular}{|c|c|}
\hline$\varepsilon_{\mathrm{s}}$ & - Deformação da armadura \\
\hline$\varepsilon_{\mathrm{y}}$ & - Deformação correspondente à tensão de escoamento \\
\hline$\phi$ & - Fator de redução de resistência \\
\hline$\phi_{\mathfrak{c}}$ & $\begin{array}{l}\text { - Inclinação da corda da barra na configuração deformada ou atual } \\
\text { em relação ao eixo global X }\end{array}$ \\
\hline$\phi_{\mathrm{r}}$ & $\begin{array}{l}\text { - Inclinação da barra na configuração de referência em relação ao } \\
\text { eixo global X }\end{array}$ \\
\hline$\gamma_{\mathrm{z}}$ & - Parâmetro global de estabilidade \\
\hline$\lambda$ & - Índice de esbeltez da coluna ou estiramento da fibra \\
\hline$\dot{\lambda}$ & - Derivada do estiramento $\lambda$ no tempo \\
\hline$\lambda_{\mathrm{c}}$ & - Fator de carga crítca \\
\hline$\rho_{\mathrm{t}}$ & $\begin{array}{l}\text { - Razão entre a área total da armadura da coluna e a área da seção } \\
\text { transversal }\end{array}$ \\
\hline$\dot{\sigma}$ & - Taxa de tensão \\
\hline$\sigma_{0}$ & - Tensão de Kirchhoff-Treffz \\
\hline$\sigma_{1}$ & - Segunda tensão de Piola-Kirchhoff \\
\hline$\sigma_{1 / 2}$ & - Tensão nominal ou de engenharia \\
\hline$\sigma_{\mathrm{c}}$ & - Tensão de Cauchy \\
\hline$\sigma_{\mathrm{KT}}$ & - Tensão de Kirchhoff-Treffz \\
\hline$\sigma_{\mathrm{N}}$ & - Tensão nominal ou de engenharia \\
\hline$\theta_{0}$ & - Rotação da corda \\
\hline$\theta_{1}$ & - Rotação do nó inicial da barra \\
\hline$\theta_{2}$ & - Rotação do nó final da barra \\
\hline$\Psi$ & $\begin{array}{l}\text { - Relação entre a rigidez das colunas e a rigidez das vigas de um } \\
\text { andar }\end{array}$ \\
\hline$\Psi_{\mathrm{A}}$ & $\begin{array}{l}\text { - Relação entre a rigidez das colunas e a rigidez das vigas para o } \\
\text { andar "A" }\end{array}$ \\
\hline$\Psi_{\mathrm{B}}$ & $\begin{array}{l}\text { - Relação entre a rigidez das colunas e a rigidez das vigas para o } \\
\text { andar "B" }\end{array}$ \\
\hline$\Psi_{\mathrm{m}}$ & $\begin{array}{l}\text { - Valor médio da relação entre a rigidez das colunas e a rigidez das } \\
\text { vigas }\end{array}$ \\
\hline
\end{tabular}




\section{RESUMO}

Neste trabalho, apresenta-se uma contribuição ao estudo da nãolinearidade física e geométrica em pórticos planos de concreto armado utilizando-se uma formulação teórica consistente.

Inicialmente, apresenta-se uma revisão dos métodos aproximados para consideração dos efeitos não-lineares em estruturas planas de concreto armado recomendados pelas principais normas internacionais.

Desenvolve-se uma formulação consistente baseada na teoria de grandes deslocamentos e grandes deformações aplicada a elemento de pórticos planos de concreto armado. Para obtenção da matriz de rigidez utiliza-se um sistema de coordenadas corrotacional solidário ao elemento, considerando-se apenas os graus de liberdade naturais. A teoria estrutural utilizada é a de Euler-Bernoulli-Navier, e as equações de equilíbrio são formuladas através do Princípio dos Trabalhos Virtuais. Para facilitar a implementação introduz-se algumas aproximações de natureza geométrica e algumas simplificações no comportamento do elemento, sem perda da generalidade da formulação.

A não-linearidade física do concreto armado é introduzida utilizando-se o processo das fatias para discretização da seção tranversal do elemento, possibilitando-se o uso de relações constitutivas uniaxiais entre tensão e deformação para o concreto e para o aço.

Foi utilizada a forma incremental do método iterativo de NewtonRaphson, permitindo-se assim acompanhar corretamente o comportamento não-linear da estrutura na fase elástica e fissurada, incluindo-se a determinação da carga de colapso.

Para verificação numérica da formulação proposta, foi implementado um programa para utilização em microcomputadores. 
Vários exemplos numéricos são apresentados e os resultados são comparados com os obtidos analiticamente e numericamente por outros autores.

Finalmente, com base em alguns exemplos de pórticos planos, é mostrada a correlação entre os resultados da análise numérica e os fornecidos pelos principais métodos aproximados de avaliação dos efeitos não-lineares recomendados pelas normas internacionais. 
In this work reinforced plane frames behavior considering material and geometric nonlinearities are investigated using a consistent finite element formulation to include large deformation and displacement effects. The element stiffness matrix is formulated in a local corotational coordinate system including only natural degrees of freedom. Equilibrium equations are formulated for the Euler-Bernoulli-Navier beam model by the virtual work method. The reinforced concrete material nonlinearity is implemented on layered plane frame elements enabling to assign one dimensional axial constitutive relations for concrete and steel materials separately. The solution is performed iteractively by the Newton-Raphson method such that the cracked region and the ultimate load can be readily identified. Numerical examples are carried out using a computer code that has been written and implemented on a microcomputer platform. The numerical results are compared with that of approximate procedures used in practice nowadays, such as P- $\Delta$ methods and moment magnifier methods, recommended by some international codes. Their results show the methodology effectiveness when compared to others available in the literature. 


\section{INTRODUÇÃo}

\subsection{Considerações Gerais}

O crescente aumento da densidade populacional associado a uma necessidade contínua de uma maior urbanização, fez com que ocorresse um intenso processo de verticalização das edificações urbanas. Com isso torna-se necessário cada vez mais o aprimoramento dos sistemas estruturais e das ferramentas de análise das estruturas, de forma a proporcionarem maior economia e uma adequada segurança.

A maioria das análises realizadas pelos projetistas estruturais são baseadas na teoria elástica-linear devido a sua simplicidade. Nesta análise considera-se uma relação linear entre as ações e os seus efeitos sobre as estruturas. Para que seja aplicável é necessário que sejam lineares as relações entre as tensões e as deformações chamadas de "linearidade física" e entre as deformações e os deslocamentos chamada de "linearidade geométrica".

A análise linear é aplicável quando se tem um nível de solicitação que produz baixas tensões e os deslocamentos são pequenos de forma que o equilíbrio possa ser formulado na posição original da estrutura.

Os resultados de uma análise linear são usualmente empregados para a verificação de Estados Limites de Utilização. Em alguns casos é possível utilizá-los, com critérios, para verificações de Estado Limite Último.

A análise linear é sempre importante pois é de mais fácil compreensão e utilização, e deve ser utilizada até mesmo como uma primeira aproximação da análise não-linear. 
A análise não-linear, embora tenha sua importância reconhecida pelos projetistas estruturais, ainda é muito pouco utilizada. O desenvolvimento de programas de análise não-linear para utilização em microcomputadores seria a forma mais adequada de introduzir esta análise no cotidiano do projeto estrutural, pois os microcomputadores estão a disposição, atualmente, da maioria dos projetistas. Uma vez vencida as dificuldades à introdução de um procedimento não convencional, a análise não-linear deverá ser reconhecida e aceita no meio profissional, pois é sem dúvida mais realista e propõe estruturas mais econômicas e seguras que a análise linear. Por estes argumentos a tendência atual das normas, inclusive a brasileira, é encorajar a utilização da análise não-linear no projeto de estruturas.

As normas técnicas internacionais, American Concrete Institute (ACI), Comité Euro-Internacional du Beton (CEB), têm recomendado uma variedade de métodos para consideração do comportamento não-linear de estruturas de concreto armado. A precisão destes métodos varia em função das hipóteses feitas com respeito ao comportamento do material (não-linearidade física) e as interações entre forças e deslocamentos nas barras da estrutura (nãolinearidade geométrica).

\subsection{Modelos de Comportamento dos Materiais}

O comportamento real dos materiais é, em geral, muito complexo, apresentando variações significativas de acordo com o tipo de solicitação (estática, dinâmica, longa ou curta duração, etc.), de acordo com as condições do meio ambiente (temperatura, umidade, etc.), ou mesmo com relação às condições de confinamento.

Assim, a análise teórica das estruturas esbarra no problema fundamental da modelagem do comportamento do material de que são 
constituídas, levando-se ao estabelecimento de relaç̃oes constitutivas $(\sigma \times \varepsilon)$ que permitam obter a solução de um determinado problema. Na prática, tornase indispensável a adoção de modelos simplificados que, no entanto, devem possibilitar soluções suficientemente aproximadas do comportamento real.

Conforme salienta PROENÇA (1986), é bastante improvável que um único modelo possa simular todas as situações possíveis, levando à necessidade da utilização de diversos modelos de um mesmo material.

Alguns modelos mais comuns são apresentados a seguir.

\subsubsection{Modelo Elástico}

A diferenciação entre os modelos se faz fundamentalmente, pela análise do regime de deformações em uma situação alternada de carga e descarga de um ensaio experimental, que em geral é o de tração simples, que fornece o diagrama $(\sigma \times \varepsilon)$.

Neste ensaio, a elasticidade fica caracterizada pelo aparecimento, numa situação de carga, de deformações imediatas, simultâneas com as tensões e invariantes ao longo do tempo se o regime de tensões permanece constante (Fig. 1.1.a e b).

Em descarga, as deformações desaparecem ao se anularem as tensões, sem deixar qualquer valor residual.

Se existe linearidade entre $\sigma$ e $\varepsilon$, o comportamento é dito elástico linear (Fig. 1.1.a), caso contrário, o regime é elástico não-linear (Fig. 1.1.b).

No caso de elasticidade linear, a proporcionalidade entre $\sigma$ e $\varepsilon$ é expressa pela Lei de Hooke,

$$
\sigma=\mathrm{E} . \varepsilon
$$

onde o módulo de elasticidade E caracteriza o material. 
$\sigma$

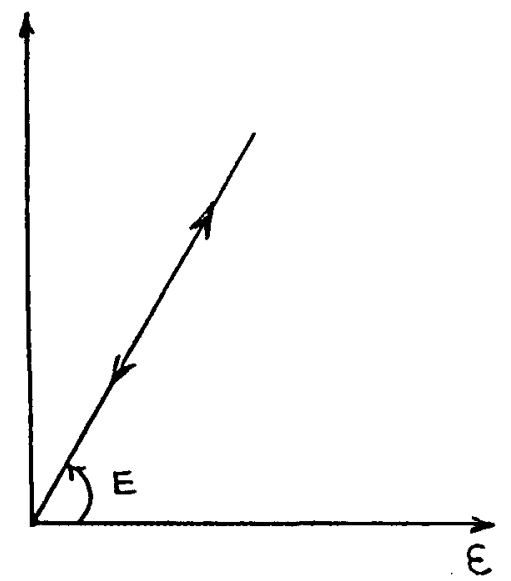

a) Regime elástico linear

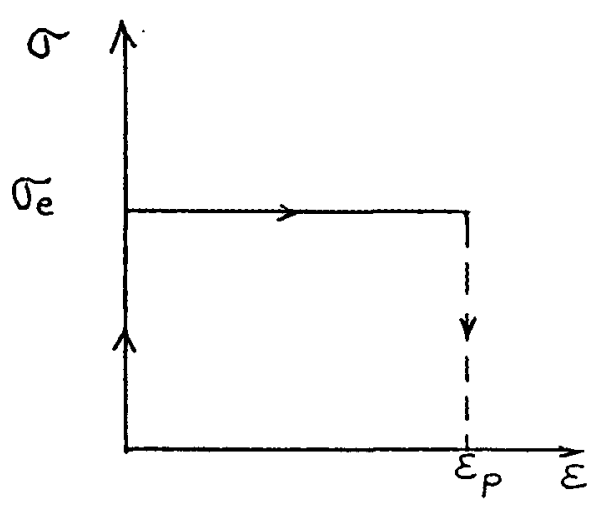

c) Regime plástico perfeito $\sigma$

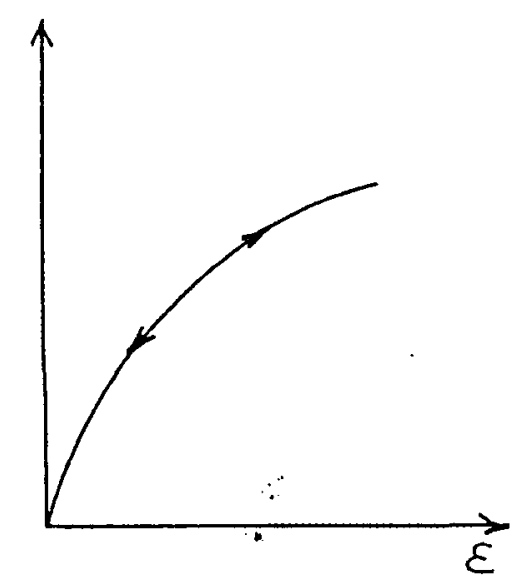

b) Regime elástico não-linear

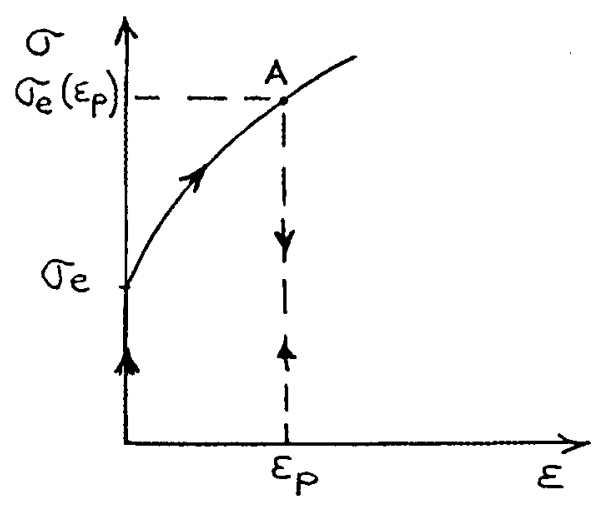

d) Regime plástico com encruamento

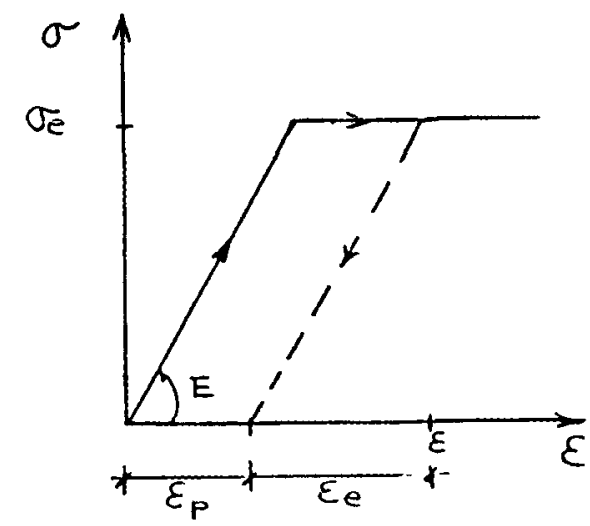

e) Regime elasto-plástico perfeito

FIGURA 1.1 - Modelos materiais idealizados 


\subsubsection{Modelo Plástico}

A plasticidade apresenta como característica principal o aparecimento de deformações residuais, não reversíveis, numa etapa de descarga.

Outra característica é relativa ao fato de que as deformações plásticas $\left(\varepsilon_{\mathrm{p}}\right)$ aparecem a partir de um determinado valor de tensão $\sigma_{\mathrm{e}}$, tensão de escoamento. Até ser atingida a tensão $\sigma_{\mathfrak{e}}, 0$ material, neste modelo, permanece rígido.

A Fig. 1.1.c mostra o comportamento plástico perfeito. Neste caso, a tensão de colapso coincide com a tensão de escoamento e, uma vez atingido este valor, a deformação ocorre de maneira ilimitada.

A Fig. 1.1.d apresenta o comportamento plástico de um material com encruamento. $\mathrm{O}$ encruamento está ligado ao fato de que o material aceita acréscimos de tensão com acréscimos de deformação plástica.

Se em uma determinada etapa do carregamento, ponto A do diagrama, houver uma descarga e em seguida uma nova carga, a tensão para a qual surgem novos acréscimos de deformação plástica é de valor diferente de $\sigma_{\mathrm{e}}$, sendo função da deformação plástica atual $\varepsilon_{\mathrm{p}}$.

\subsubsection{Modelo Viscoso}

Embora a viscosidade seja uma característica dos líquidos, nos sólidos ela se manifesta pelo aparecimento de deformações que se desenvolvem ao longo do tempo, mesmo que as tensões permaneçam constantes.

No tratamento analítico deste tipo de comportamento, admite-se que a tensão $\sigma$ é proporcional a uma taxa de deformação $\dot{\varepsilon}$, de tal modo que ao se aplicar a tensão, não aparece, de imediato, uma deformação.

A relação constitutiva fundamental é 


$$
\sigma=\eta \dot{\varepsilon}
$$

onde $\eta$ é o coeficiente de viscosidade do material.

A simulação da deformação ao longo do tempo pela equação 1.2 , fica evidenciada na resolução do caso particular de solicitação onde se admite que $\sigma$ permaneça constante e igual a $\bar{\sigma}$. Considerando-se também que em $t=0$, $\varepsilon(0)=0$, a equação 1.2 integrada conduz a

$$
\varepsilon(t)=\frac{\bar{\sigma}}{\eta} t
$$

A equação 1.2 permite ainda uma outra consideração. Se em um determinado instante for removida a tensão $\bar{\sigma}$, a partir daí $\dot{\varepsilon}=0$ e, portanto, $\varepsilon$ é constante. Isto é, aparece uma deformação não reversível diferente daquela do modelo plástico pelo fato de não ser imediata.

\subsubsection{Modelos Combinados}

Os três modelos básicos anteriores (elástico, plástico e viscoso) podem ser combinados segundo diversos critérios, de modo a permitir o estudo do comportamento de diferentes materiais, os quais apresentam, em geral, deformações elásticas, plásticas e viscosas.

Essas combinações podem ser agrupadas em quatro grupos:

- modelo Elasto-Plástico;

- modelo Visco-Elástico;

- modelo Visco-Plástico;

- modelo Elasto-Viscoplástico. 
O modelo elástico perfeitamente plástico é mostrado na Fig. 1.1.e. Enquanto a tensão $\sigma<\sigma_{e}$, a deformação total é composta apenas da parcela elástica; quando $\sigma=\sigma_{\mathrm{e}}$, a deformação total é obtida pela soma da parcela elástica e da parcela plástica, ou seja:

$$
\varepsilon=\varepsilon_{\mathrm{p}}+\varepsilon_{\mathrm{e}}
$$

Neste modelo, o descarregamento ocorre elasticamente.

O comportamento do material visco-elástico é usualmente exibido na forma de diagrama de fluência e relaxação. A fluência ou creep é o fenômeno do aparecimento de deformações ao longo do tempo, sendo o corpo solicitado por tensão constante. Por outro lado, a relaxação se caracteriza por uma perda de tensão ao longo do tempo, em um corpo sujeito a deformação constante.

$\mathrm{O}$ modelo visco-plástico permite simular o comportamento de materiais que apresentam deformações plásticas e viscosas. Basicamente, o material se comporta como rígido até atingir a tensão de escoamento e, a partir daí, as propriedades plásticas e viscosas são evidenciadas.

O material elasto-viscoplástico tem um comportamento elástico até atingir a tensão de escoamento e, a partir daí, deformações plásticas e viscosas são adicionadas às deformações elásticas. Estas deformações viscoplásticas são resultantes da tensão que excede a tensão de escoamento.

\subsection{Interação Entre Forças e Deslocamentos}

Quando submetidas a ação de forças aplicadas a estrutura deforma, implicando-se que as forças devem satisfazer as condições de equilíbrio na posição deformada (Fig. 1.2). Como em geral estas deformações são pequenas 
comparadas com as dimensões da estrutura, estas tem sido desprezadas na formulação do equilíbrio levando-se à chamada análise em teoria de $1^{\mathrm{a}}$ ordem.

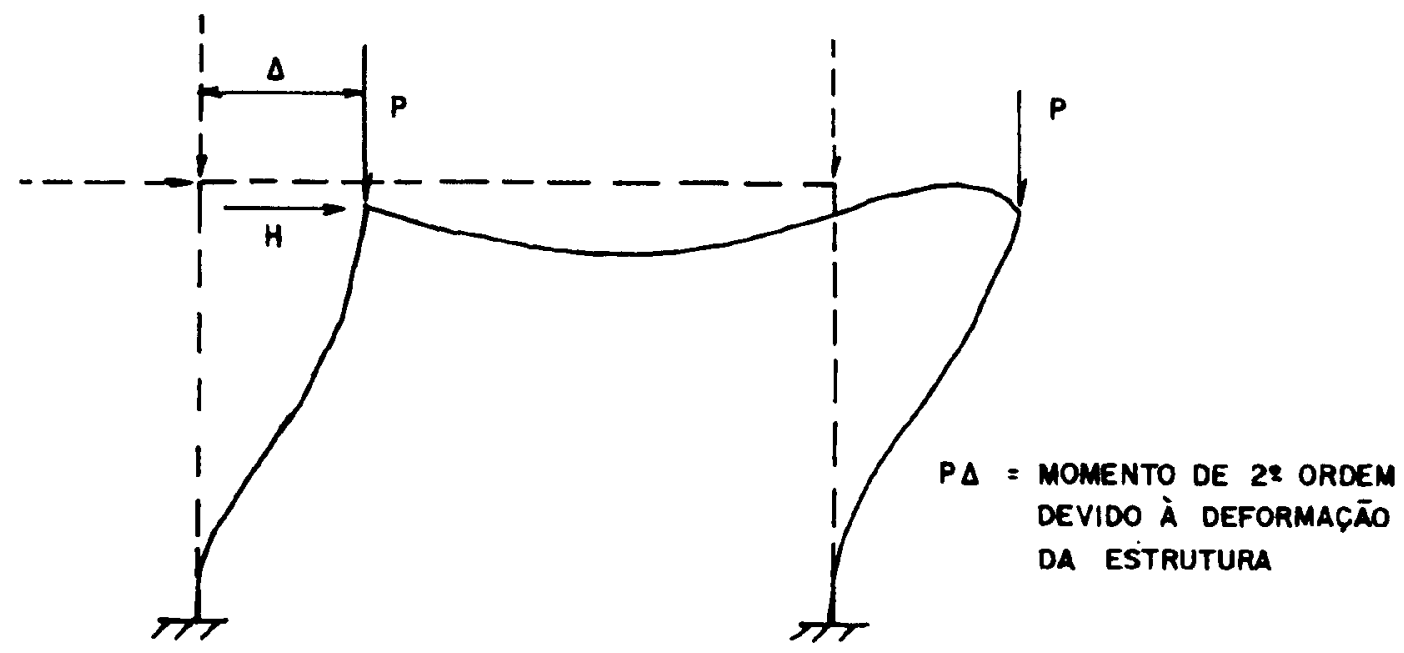

FIGURA 1.2 - Efeito P- $\Delta$

Se o equilíbrio é formulado na estrutura em sua posição deformada, levando-se em consideração momentos adicionais devido a forças axiais, a análise é dita em teoria de $2^{\mathrm{a}}$ ordem.

Existem situações em que a utilização da teoria de $2^{\mathrm{a}}$ ordem é essencial, principalmente na análise de estruturas esbeltas de edifícios e onde se deseja analisar mecanismos e possibilidades de colapso ou determinar a real reserva de segurança para um dado nível de carregamento.

\subsection{Tipos de Análises de Pórticos}

Diferentes combinações das hipóteses descritas abaixo levam a uma variedade de curvas relacionando-se carregamento e deslocamento como mostrado na Fig. 1.3. Embora a figura mostre relações entre o carregamento e 
o deslocamento lateral para um pórtico simples de duas colunas e uma viga, os resultados podem ser extrapolados para pórticos de vários vãos e andares múltiplos.

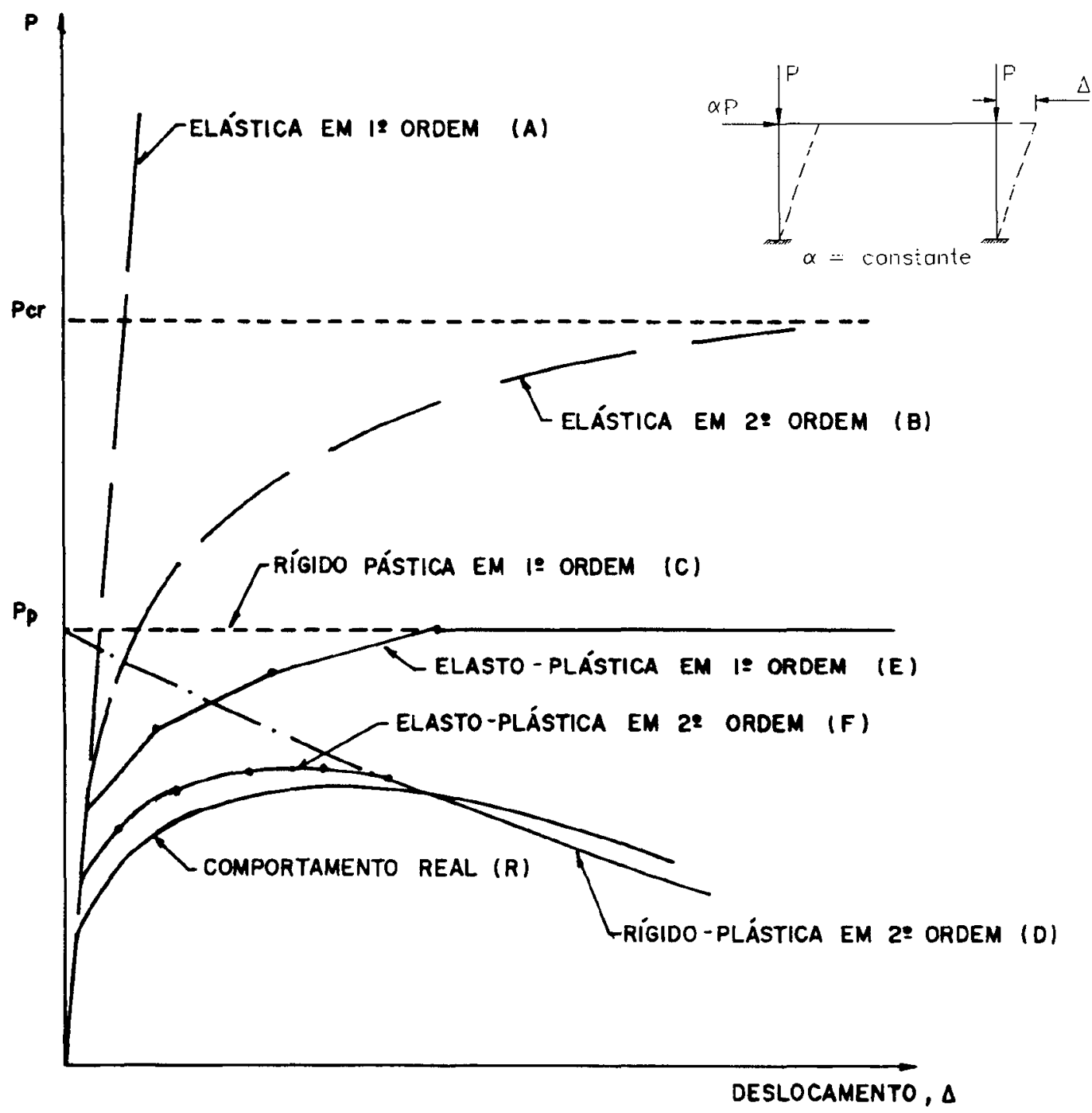

FIGURA 1.3 - Relações carga-deslocamento para pórticos

A forma mais simples de analisar a estrutura de um pórtico é através da análise elástica linear em $1^{\text {a }}$ ordem. Nesta análise o material é assumido ser elástico linear, ou seja segue a lei de Hooke, e as equações de equilíbrio são 
formuladas baseando-se na configuração indeformada da estrutura. A curva "A" da Fig. 1.3 representa os resultados deste tipo de análise.

Este tipo de análise é de muito importância por várias razões como as relacionadas a seguir e citadas por CORREAA (1991):

- é a análise de mais fácil compreensão e utilização;

- possibilita a superposição de efeitos, que simplifica muito a análise;

- em regime de serviço é comum grande parte dos pórticos de edifícios ter comportamento marcadamente linear;

- mesmo em situações em que a não-linearidade é marcante, a análise deve ser utilizada como primeira aproximação do comportamento global do pórtico;

- vários tipos de análises não-lineares podem ser desenvolvidas por sucessivas etapas lineares.

Se as equações de equilíbrio são formuladas baseando-se na configuração deformada obtem-se a curva "B", a qual representa uma análise elástica em $2^{\mathrm{a}}$ ordem. O limite superior da análise elástica em $2^{\mathrm{a}}$ ordem, para pequenas deformações, é a carga de flambagem elástica do pórtico, $\mathrm{P}_{\mathrm{cr}}$.

Se as equações de equilíbrio são referidas a forma indeformada da estrutura e o material tem comportamento rígido-plástico obtem-se a resposta representada pela curva " $\mathrm{C}$ ", e esta análise é denominada análise em $1^{\mathrm{a}}$ ordem em regime rígido-plástico. Formulando-se o equilibrio na posição deformada da estrutura a curva "D" da Fig. 1.3 é obtida, e a análise é dita rígido-plástica em $2^{\mathrm{a}}$ ordem.

$\mathrm{Na}$ análise elasto-plástica em teoria de $1^{\mathrm{a}}$ ordem o comportamento do material é assumido como elástico até um certo limite e a partir daí perfeitamente plástico e o equilíbrio é formulado na posição inicial da estrutura. A resposta carga-deslocamento para este tipo de análise é mostrada pela curva " $E$ " e o seu limite superior é a carga de colapso plástico, $P_{c}$, obtida da análise rígido-plástica em $1^{\mathrm{a}}$ ordem. Se o equilíbrio for formulado na 
configuração deformada, obtém-se a curva "F", a qual representa uma análise elasto-plástica em $2^{\mathrm{a}}$ ordem, também conhecido como análise não-linear em $2^{\mathrm{a}}$ ordem. Esta análise é uma boa aproximação do comportamento real da estrutura de um pórtico, representado pela curva "R" da Fig. 1.3.

Embora a análise linear seja a mais utilizada nos escritórios de projetos, há situações em que a utilização de análises não-lineares é essencial para se obter resultados próximos dos reais; algumas delas são citadas a seguir:

- análise de estruturas esbeltas de edifícios, onde os efeitos de $2^{\mathrm{a}}$ ordem (não linearidade geométrica) têm influência significativa;

- em estruturas de concreto armado onde o nível de tensões leve à fissuração do concreto, plastificações e a outros comportamentos não-lineares deste material;

- em estruturas de comportamento elasto-plástico;

- quando se deseja analisar mecanismos e possibilidades de colapso, reservas de segurança para um dado nível de carregamento, etc.

A diferença entre as curvas obtidas da análise elasto-plástica em $2^{\mathrm{a}}$ ordem e a curva do comportamento real do pórtico depende de muitos fatores, tais como:

- imperfeições iniciais das barras

- efeito das deformações por cisalhamento

- influência dos efeitos não-lineares do material

- etc.

\subsection{Considerações Sobre o Comportamento do Concreto Armado}

As estruturas de concreto armado apresentam um comportamento complexo em função das propriedades de seus materiais componentes (aço e 
concreto) e do trabalho destes em conjunto (aderência), levando-o a respostas não-lineares.

O concreto é um material heterogêneo que se encontra em intercâmbio hidráulico constante com o meio ambiente, o que faz com que as reações químicas assim produzidas alterem suas propriedades ao longo do tempo. Entre as características importantes do material concreto CILONI (1993) cita:

-boa resistência à compressão e baixa resistência à tração, o que leva à sua fissuração;

-existência de deformações diferidas produzidas ou não por tensões (fluência, retração);

- influência do tipo e da duração da solicitação na resistência do material (estado uniaxial ou multiaxial de tensões, efeito RUSH, fadiga, etc.);

-baixa ductilidade do material, se comparado com o aço, com deformações últimas muito baixas;

- relação não-linear entre tensões de compressão e deformações, com a existência de um valor máximo e de um ramo descendente;

- comportamento sob cargas dinâmicas com ciclos de histerese, degradação da rigidez e da resistência do concreto.

Já o aço apresenta outras características próprias:

- resistência semelhante à tração e à compressão;

- comportamento inicialmente elástico, com módulo sensivelmente constante, com fase plástica posterior, esta com características dependentes do tipo de aço (A ou B);

- ausência de fluência e retração;

- efeito BAUSCHINGER sob ação de ciclos de cargas. 
Trabalhando em conjunto o concreto e aço apresentam outras características importantes, como por exemplo:

-aderência imperfeita entre as armaduras e 0 concreto, com deslizamentos relativos entre ambos, que tendem a aumentar sob atuação de ciclos de carga e descarga;

- contribuição do concreto tracionado entre fissuras.

- coeficientes de dilatação térmica aproximadamente iguais.

É evidente que a natureza não-linear da relação momento-curvatura para as seções das barras de uma estrutura de concreto armado provocará algumas alterações nos valores relativos dos esforços solicitantes obtidos numa análise elástica em $1^{\mathrm{a}}$ ordem. Em particular por causa das rotações plásticas de algumas seções é possível que os momentos fletores assumam valores diferentes daqueles obtidos da análise linear, e ao atingir os valores limites, o efeito da fissuração provoca uma redistribuição dos momentos em estruturas estaticamente indeterminadas.

Esta redistribuição de momentos depende da capacidade de rotação plástica de cada seção. Se a seção é frágil o momento decresce rapidamente após atingir o máximo e a estrutura romperá bruscamente sem absorver qualquer carga adicional. Já se a seção é dúctil, cargas adicionais podem ser aplicadas porque rotações ocorrem enquanto o momento resistente permanece constante e uma redistribuição de momentos ocorrerá, e assim sucessivamente até que o mecanismo se forme. Portanto se existe capacidade de absorver rotações plásticas a distribuição do momentos para a carga de ruptura pode ser completamente diferente daquela obtida usando a análise elástica linear e dependerá dos momentos últimos das seções. Em estruturas de concreto armado a ductibilidade de uma barra é usualmente expressada como a razão entre a deformação última e a deformação correspondente à primeira plastificação. A ductibilidade para a formação da $1^{\mathrm{a}}$ rótula plástica pode ser 
insuficiente para possibilitar a total redistribuição dos momentos. Assim, se a redistribuição é pretendida deve-se assegurar suficientes ductibilidades das seções críticas.

Outra não-linearidade que ocorre nas barras das estruturas de concreto armado é devida à variação da rigidez EI ao longo de seu comprimento. Quando a barra fissura a rigidez EI da seção fissurada reduz e esta variação de EI ao longo da barra altera a distribuição dos esforços solicitantes se comparada com a análise elástica (EI constante). Com o aumento do carregamento a extensão da região fissurada aumenta e a distribuição da rigidez, assim como dos esforços solicitantes, será outra vez modificada. Este efeito é mais acentuado quando as diversas barras da estrutura contém diferentes densidades de armaduras positivas e negativas, e é mais notada ainda nos pórticos porque a fissuração é maior nas vigas do que nos pilares.

Como exemplo é mostrada uma viga hiperestática sobre três apoios (Fig. 1.4): na seção do apoio central, onde é maior o momento negativo, a fissuração dessa seção provoca uma redução na rigidez e conseqüentemente uma redistribuição de momentos, com o aumento dos momentos positivos e a antecipação da fissuração nos vãos em relação à previsão da teoria elástica.

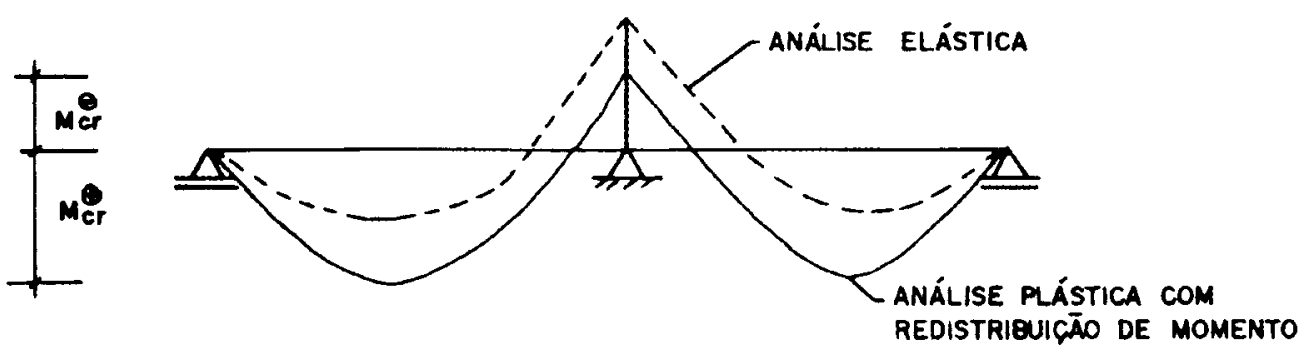

FIGURA 1.4 - Redistribuição de momentos provocada pela fissuração numa viga hiperestática

As dificuldades envolvidas na consideração dos fenômenos comportamentais do concreto armado levaram, no passado, os projetistas de 
estruturas de concreto a projetar baseando-se em fórmulas empíricas derivadas de numerosos experimentos. Com o advento dos computadores permitiu-se grande avanço nos métodos numéricos de análise, como por exemplo o método dos elementos finitos, e o conseqüente desenvolvimento de modelos matemáticos mais realísticos com os quais se tenta descrever com mais precisão o comportamento das estruturas de concreto armado. Obviamente estas soluções numéricas precisam ser confrontadas com dados experimentais, assim, como, as análises numéricas devem ser utilizadas para aferir resultados obtidos nos laboratórios.

\subsection{Considerações Gerais Sobre os Métodos de Projetos em Concreto} Armado

As normas mais modernas de projeto de estruturas de concreto armado já incorporam em suas prescrições diferentes tipos de análise que podem ser feitas dentro de um projeto. Assim é que o Código Modelo CEB/FIP MC 90 (1990), explicita em seu parágrafo 5.3 quais as análises que podem ou devem ser feitas. Diz o texto:

\footnotetext{
"A análise global de uma estrutura pode ser feita de acordo com os seguintes métodos: análise não-linear, análise linear, análise linear com redistribuição e análise plástica."
}

O texto especifica que a análise não-linear é uma descrição realista do comportamento físico e por conseguinte um método perfeitamente consistente com as hipóteses usadas para verificações locais e cálculo dos elementos que compõem a estrutura. Enfatiza que este método deve ser usado como referência para outros mais simplificados. Com relação à análise linear o $\mathrm{MC}$ 90 observa que ela só é realista quando a estrutura está submetida a baixos 
níveis de tensão e se seus elementos ainda não estão fissurados. A análise linear com redistribuição de esforços, todavia limitada, pode ser utilizadas para verificações na ruptura (Estado Limite Último).

Interpretando as afirmações do MC 90, MARTINS (1995) observa que o método clássico de projeto baseado na obtenção de esforços via análise linear e posterior verificação das seções nos estados limites últimos com esses esforços carece de consistência e tem sua abrangência extremamente limitada. Conforme frisa o MC 90 pode-se aceitá-la para cálculo de vigas contínuas e pórticos indeslocáveis lateralmente. Ocorrendo fissuração, até mesmo para verificações dos estados limites de utilização, a análise linear é teoricamente deficiente.

Cabe aqui ressaltar que sempre se calculou obras de concreto armado baseadas em cálculos lineares sem que isto implicasse em danos à estrutura. Mesmo sendo verdade esta observação, isto não invalida o fato de seus resultados serem questionáveis e de que as margens efetivas de segurança possam não ser as previstas nos cálculos.

A consideração do comportamento não-linear dos pórticos de concreto armado de edifícios pode ser feita através de métodos mais precisos como a análise não-linear fisica e geométrica utilizando-se para isto programas de elementos finitos ou através de métodos simplificados como o método P- $\Delta$ ou os métodos de comprimento efetivo de coluna.

O ACI 318-89 (1989) sugere que se faça uma análise levando em conta a influência das forças axiais e das variações de inércia devido à fissuração na rigidez das barras dos pórticos de edificios, assim como os efeitos decorrentes das cargas permanentes e das rotações de apoio na estrutura lateralmente deslocada devido às ações do vento.

O CEB/FIP MC 90 é mais exigente que o ACI 318-89, ele não apenas incentiva o uso de análises que incluam as reais leis constitutivas dos materiais, mas também fornece aos projetistas técnicas de como levar em 
consideração as não-linearidades geométricas e as variações de rigidez devido a fissuração.

Dentre os métodos simplificados de projeto recomendados pelas normas pode-se citar os métodos de comprimentos efetivos, onde as considerações de não-linearidades são levadas em conta diretamente no dimensionamento das colunas. $O$ método de amplificação dos momentos de $1^{\mathrm{a}}$ ordem recomendado pelo ACI 318-89; o método do momento adicional do CEB/FIB MC 90; o método do aumento da excentricidade devido ao efeito da esbeltez prescrito pela NBR 6118 (1980) são exemplos destes métodos de comprimento efetivo de coluna.

Outros métodos simplificados muito utilizados nos escritórios de projetos são os chamados "Métodos P- $\Delta$ ", que consistem basicamente em simular os efeitos de $2^{\mathrm{a}}$ ordem (não-linearidade geométrica) através de forças cortantes e momentos fletores adicionais produzidos pelo carregamento vertical agindo na estrutura lateralmente deslocada. Estes esforços são obtidos usando apropriadas rigidezes EI para computar a fissuração do concreto.

Os parâmetros de estabilidade, definidos por BECK \& KONIG (1966), MACGREGOR \& HAGE (1977), FRANCO (1985) e VASCONCELOS \& FRANCO (1990), surgem como indicadores da estabilidade global da estrutura. Basicamente eles informam se o pórtico pode ser considerado como de nós fixos ou de nós móveis, sendo que nos pórticos de nós fixos os efeitos de $2^{\mathrm{a}}$ ordem podem ser considerados em cada pilar isoladamente em função do seu índice de esbeltez, utilizando-se um dos métodos de comprimento efetivo citados anteriormente.

Dentre estes parâmetros de estabilidade, um dos mais utilizados é o chamado parâmetro $\alpha$, inicialmente definido por BECK \& KONIG baseado na análise de pórticos articulados contraventados por uma parede estrutural e dado por: 


$$
\alpha=\mathrm{h} \sqrt{\frac{\mathrm{P}}{\mathrm{EI}}}
$$

onde $\mathrm{h}$ é a altura total do pórtico, $\mathrm{P}$ é a carga vertical total atuante, EI é o produto de rigidez da parede de contraventamento suposto constante ao longo da altura. Se $\alpha<0,6$ dispensa-se a análise global de $2^{\mathrm{a}}$ ordem da estrutura.

FRANCO entende que o critério de equivalência da rigidez possa ser estendido a estruturas de contraventamento compostas apenas por paredes ou apenas por pórticos. Como os efeitos de $2^{\mathrm{a}}$ ordem dependem da forma da elástica da estrutura, ele sugere diferentes valores limites de $\alpha$ para os diversos tipos de contraventamentos:

$-\alpha \leq 0,5$ para estruturas compostas apenas por pórticos;

$-\alpha \leq 0,6$ para estruturas compostas por pórticos e paredes;

$-\alpha \leq 0,7$ para estruturas compostas apenas por paredes.

Mais recentemente VASCONCELOS \& FRANCO mostraram que a avaliação da deslocabilidade lateral da estrutura de contraventamento pode ser realizada de forma muito conveniente pelo parâmetro $\gamma_{\mathrm{z}}$, dado por:

$$
\gamma_{z}=\frac{1}{1-\frac{\Delta M}{M_{1}}}
$$

onde $\Delta \mathrm{M}$ é o incremento inicial do momento de $1^{\frac{a}{}}$ ordem causado pela ação das cargas verticais na estrutura lateralmente deslocada.

Esse parâmetro pode ser utilizado para estimar o acréscimo que se deve dar ao momento de $1^{a}$ ordem para se obter o momento que inclui também a parcela de $2^{\mathrm{a}}$ ordem, ou seja:

$$
\mathrm{M}_{2}=\gamma_{\mathrm{z}} \cdot \mathrm{M}_{1}
$$


Assim, considerando-se que a parcela de $2^{\mathrm{a}}$ ordem deve ser de no máximo $10 \%$ da de $1^{\mathrm{a}}$ ordem para que a estrutura possa ser considerada como indeslocável, tem-se que o limite do parâmetro $\gamma_{2}$, para esse caso deve ser:

$$
\gamma_{z} \leq 1,1
$$

CORRÊA \& RAMALHO (1995) mostram que pode-se trabalhar com este coeficiente de majoração $\gamma_{Z}$ até o limite de 1,2. É mostrado ainda pelos mesmos autores que existe uma boa correlação entre os parâmetros $\alpha$ e $\gamma_{z}$, calculados de mais de 60 pares de valores $\alpha$ e $\gamma_{z}$, obtidos para edifícios projetados e executados no Brasil, chegou-se à seguinte correlação:

$$
\gamma_{z}=1,1-0.33 \alpha+0.50 \alpha^{2}
$$

MACGREGOR \& HAGE (1977) definem um outro parâmetro de estabilidade $Q$, a partir da consideração de que se os momentos de $2^{\mathrm{a}}$ ordem não diferem em mais de $5 \%$ dos de $1^{\text {a }}$ ordem, a análise em $2^{\text {a }}$ ordem é dispensável; assim ele define $\mathrm{Q}$ como:

$$
\mathrm{Q}=\frac{\left(\sum \mathrm{P}\right) \Delta_{1}}{\mathrm{H} \cdot \mathrm{h}}
$$

onde $\Sigma \mathrm{P}$ é a resultante das cargas verticais no andar, $\Delta_{1}$ é o deslocamento lateral relativo de $1^{\mathrm{a}}$ ordem do andar, $\mathrm{H}$ é a força cortante no andar e $\mathrm{h}$ é a altura do andar. MACGREGOR \& HAGE então admitem que se Q $\leq 0,0475$ é desnecessária a análise em $2^{\mathrm{a}}$ ordem, e recomendam ainda que se possa fazer análise simplificada em $2^{\mathrm{a}}$ ordem (análise do tipo $\mathrm{P}-\Delta$ ) para valores $\mathrm{Q} \leq 0,22$. 
A revisão da NBR 6118 em andamento deve incluir recomendações sobre considerações dos parâmetros de estabilidade e de processos simplificados de consideração dos efeitos não-lineares em estruturas de concreto armado.

No capítulo II será apresentada uma revisão das prescrições normativas de projeto recomendados pelas diversas normas internacionais no que se refere a consideração dos efeitos das não-linearidades em pórticos planos de concreto armado.

\subsection{Objetivos do Trabalho}

O projeto de uma estrutura de concreto armado deve atender com segurança os estados limites últimos e os estados limites de utilização. Para assegurar o atendimento às condições de serviço, é necessário uma previsão rigorosa dos esforços solicitantes, deslocamentos e deformações da estrutura sob ação das cargas de utilização. É importante para se conhecer as reais condições de segurança estimar com precisão também a carga última da estrutura. Portanto um projeto consistente de uma estrutura deve procurar antecipar o comportamento mais próximo possível do real, nas fases elástica, fissurada e nas vizinhanças da ruptura.

Com o objetivo de melhor conhecer o comportamento dos pórticos planos de concreto armado nas diversas fases de carregamento, neste trabalho pretende-se estudar o comportamento destes pórticos através de uma análise não-linear em teoria de $2^{\mathrm{a}}$ ordem, baseado na teoria de grandes deslocamentos e grandes deformações usando para isto uma formulação teórica consistente.

O trabalho envolve o desenvolvimento da formulação para análise não-linear de pórticos planos e a elaboração de um programa para utilização em microcomputadores para análise não-linear de pórticos planos de múltiplos 
andares em concreto armado, levando-se em consideração a deformabilidade dos apoios. Esta formulação consistente é baseada nos trabalhos desenvolvidos por PIMENTA (1986 a 1995), e para consideração da não-linearidade fisica do concreto armado é utilizado o processo das fatias que permite o uso de relações constitutivas uniaxiais entre tensão e deformação para o concreto e para o aço. Neste processo as seções transversais das barras de concreto armado são divididas em fatias de concreto e de aço, podendo-se portanto ser utilizado para seções de forma qualquer, com várias camadas de armadura.

É também objetivo deste trabalho incluir um estudo comparativo entre os resultados numéricos com aqueles obtidos por métodos aproximados usados em projetos atendendo às prescrições das várias normas internacionais, tais como, o método P- $\Delta$ e o método de amplificação de momentos de $1^{\mathrm{a}}$ ordem, assim como as recomendações relativas a redução da rigidez EI para levar em consideração a físsuração do concreto.

\subsection{Descrição Resumida dos Capítulos}

No capitulo II apresenta-se uma revisão das prescrições normativas de projeto recomendadas pelo ACI, CEB/FIB e NBR 6118 no que se refere a consideração dos efeitos não-lineares tanto geométricos quanto físicos, para pórticos planos de concreto armado de edifícios de andares múltiplos.

No capítulo III é apresentada de maneira detalhada a formulação para análise não-linear de pórticos planos, baseada na teoria de grandes deslocamentos e grandes deformações. A aplicabilidade da formulação na análise de grandes deslocamentos é ilustrada através de vários exemplos e os resultados são comparados com os obtidos através de soluções analíticas. No capítulo IV a formulação é aplicadas à análise de pórticos planos de concreto armado, onde são apresentados modelos constitutivos para o concreto e o aço. 
Apresenta-se também um fluxograma simplificado do programa principal e das diversas subrotinas com a descrição suscinta dos vários passos de uma análise não-linear incremental e iterativa. Vários exemplos são apresentados com 0 objetivo de se mostrar a validade da formulação e de se comparar os resultados com os obtidos quando se utiliza os procedimentos aproximados de análise não-linear recomendados pelas diversas normas.

Finalmente no capítulo $\mathrm{V}$ são enfocados as principais conclusões extraídas deste trabalho e algumas sugestões para trabalhos futuros. 


\section{PRESCRIÇÕES NORMATIVAS PARA ANÁLISE NÃO- LINEAR DE PÓRTICOS EM CONCRETO ARMADO}

\subsection{Generalidades}

As normas mais modernas de projeto de estruturas de concreto armado já incorporam em suas prescrições diferentes métodos de análise não-linear. Aqui serão comentadas as recomendações do ACI-318/318R-89, do CEB/FIP Manual of buckling and stability (1978) e da NBR-6118 (1980).

Pode-se avaliar os efeitos não-lineares em pórticos planos de concreto armado de edifícios de andares múltiplos através de 3 procedimentos possíveis:

a)Métodos de análise não-linear;

b)Métodos P- $\Delta$;

c)Métodos baseados no comprimento efetivo de coluna.

\subsection{Métodos de Análise Não-linear}

Segundo o ACI 318-89 o projeto de barras de pórticos deverá ser baseado nos esforços solicitantes majorados obtidos de uma análise em $2^{\mathrm{a}}$ ordem considerando a não-linearidade do material, fissuração assim como os efeitos da curvatura das barras, inclinação lateral, duração do carregamento, 
retração, deformação lenta e interação com as fundações dos apoios. O procedimento de análise deverá apresentar resultados respaldados naqueles obtidos em laboratórios.

$\mathrm{ACI}$ incentiva o uso da análise não-linear considerando os efeitos supracitados para pórticos deslocáveis por causa dos grandes efeitos na estabilidade causados pelos deslocamentos laterais dos nós. Para obter valores precisos de deslocamentos e esforços de $2^{\mathrm{a}}$ ordem deve-se dar ênfase à utilização de valores realísticos das rigidezes da barra.

O CEB/FIP é mais ousado que o ACI. Ele não só incentiva o uso de análises que incluem as leis constitutivas dos materiais ou relações momentocurvatura para as seções como função do tempo, e os efeitos das ações nos deslocamentos, como fornece aos projetistas, técnicas de como fazer tal análise, incluindo como considerar as variações de rigidez das seções.

As duas principais dificuldades na análise de pórticos esbeltos em concreto armado são as considerações das não-linearidades físicas e geométricas. Estas dificuldades tendem a diminuir com o surgimento de programas que considerem estas não-linearidades, o que hoje ainda é pouco acessível à maioria dos escritórios de projetos.

\subsection{Métodos P- $\Delta$}

A estabilidade de um pórtico depende principalmente da interação entre as forças verticais e os deslocamentos laterais. Esta interação afeta não só as colunas mas também as vigas. Sendo assim os projetos de pórticos demandam modelos mais realísticos do que analisar colunas isoladas considerando-se apenas deslocamentos dos andares.

No ítem 2.1, foi mencionado que uma análise não-linear mais precisa deveria incluir não-linearidade geométrica, não-linearidade física do material e efeitos reológicos. Neste ítem, métodos aproximados, mas que consideram o 
comportamento global do pórtico, serão discutidos. Nesses métodos comumente chamados de métodos $P-\Delta$ serão considerados os efeitos da nãolinearidade geométrica através da aplicação das forças horizontais equivalentes produzidas pela interação do carregamento vertical na estrutura lateralmente deslocada. Os efeitos da não-linearidade física são usualmente considerados usando-se valores aproximados para EI.

Embora a análise não-linear em teoria de $2^{\mathrm{a}}$ ordem seja a maneira mais adequada para a determinação dos reais deslocamentos e esforços solicitantes em pórticos em concreto armado, tal análise pode ser muito complexa e as vezes inacessível a maioria dos escritórios de projetos. Assim análises aproximadas através dos métodos P- $\Delta$ podem ser utilizadas através de modificações na análise linear.

\subsubsection{Análise $\mathbf{P}-\Delta$ Iterativa}

Os esforços e os deslocamentos em $2^{\mathrm{a}}$ ordem são obtidos através de cálculo iterativo, usando-se um programa de análise em $1^{\mathrm{a}}$ ordem, simulandose o efeito P- $\Delta$ através de esforços fictícios, como se mostra a seguir.

Inicialmente efetua-se uma análise em $1^{\mathrm{a}}$ ordem do pórtico sob ação de forças verticais e horizontais. Desta análise pode-se determinar os deslocamentos laterais de cada andar, e portanto os deslocamentos relativos $\Delta_{1}^{\mathrm{j}}$ (Fig. 2.1).

O próximo passo é calcular a resultante das forças axiais nas colunas $\left(\Sigma P_{j}\right)$ em cada andar e conseqüentemente determinar-se as forças cortantes adicionais em cada andar $\left(\mathrm{V}_{1}^{\mathrm{j}}\right)$ como:

$$
\mathrm{V}_{1}^{\mathrm{j}}=\left(\sum \mathrm{P}^{\mathrm{j}}\right) \Delta_{\mathrm{i}}^{\mathrm{j}} / \mathrm{h}_{\mathrm{j}}
$$


onde $h_{\mathrm{j}}$ é a altura do andar.

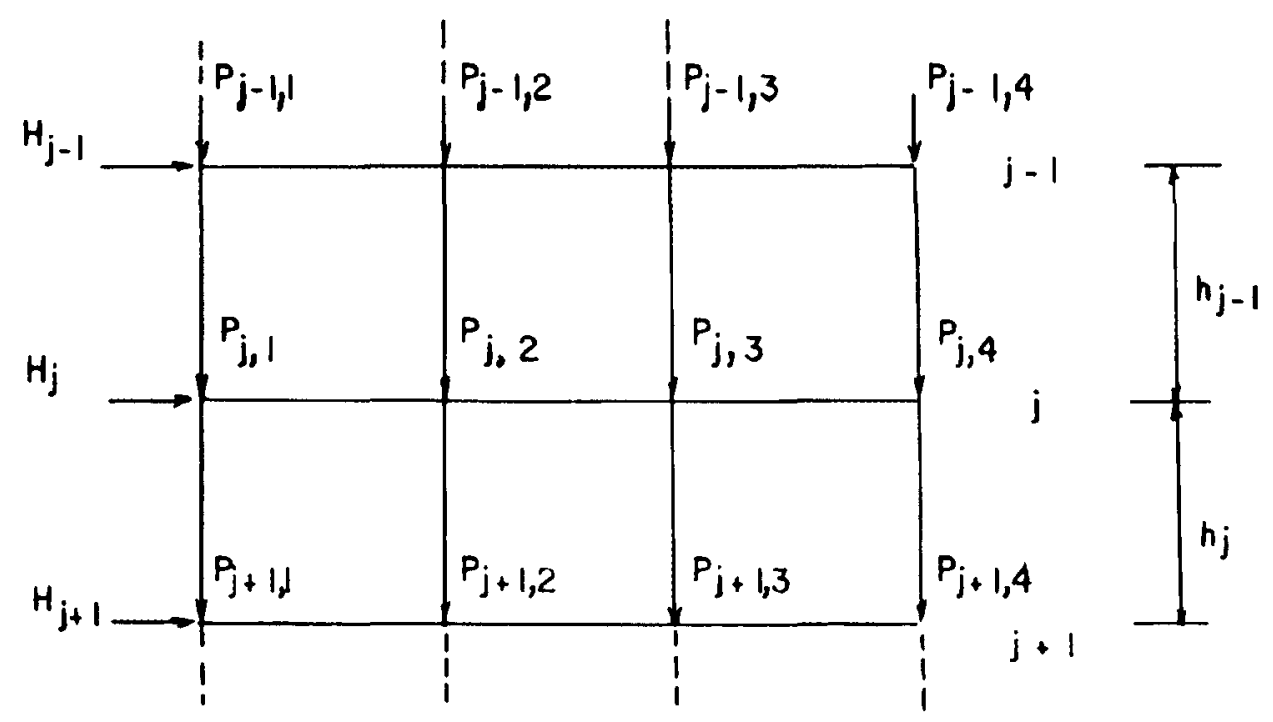

(a) ANÁLISE EM 1? ORDEM.

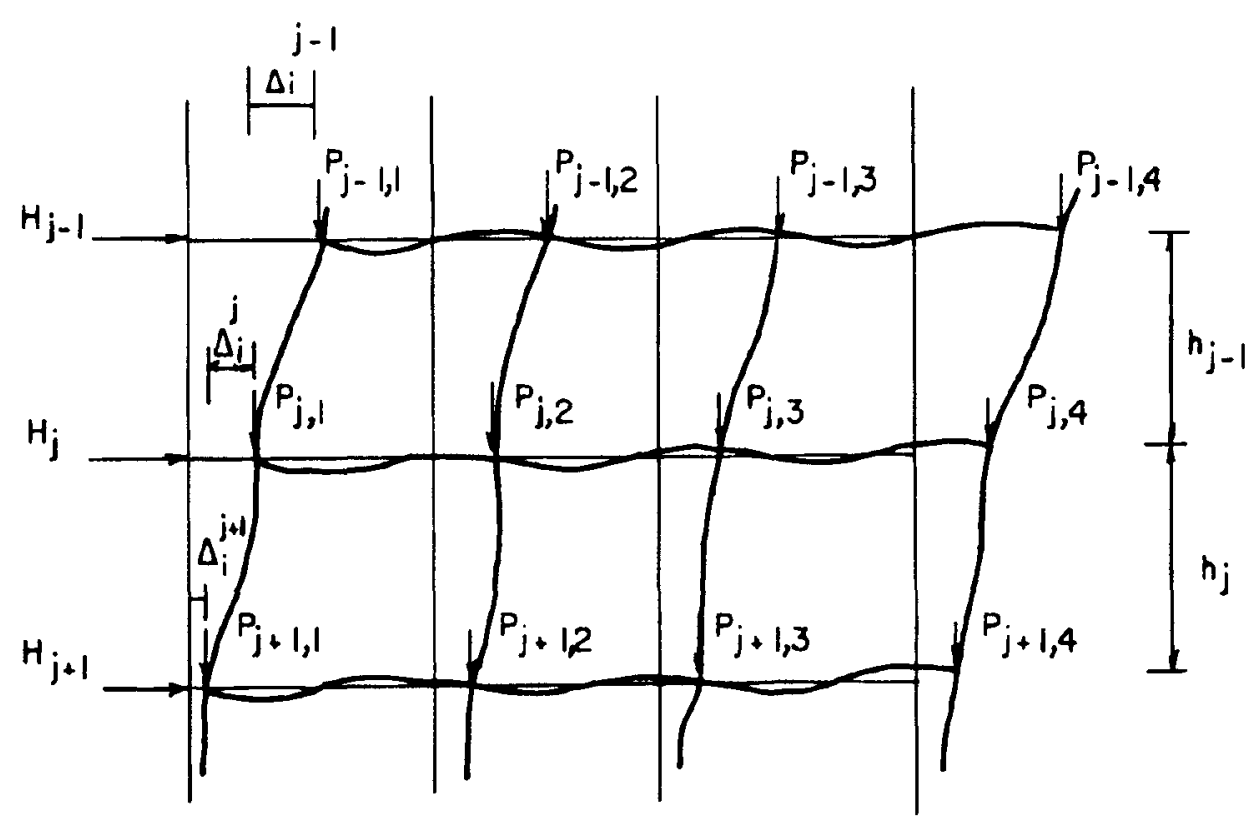

(b) DESLOCAMENTO DE 19 ORDEM.

FIGURA 2.1 - Método P- $\Delta$ 

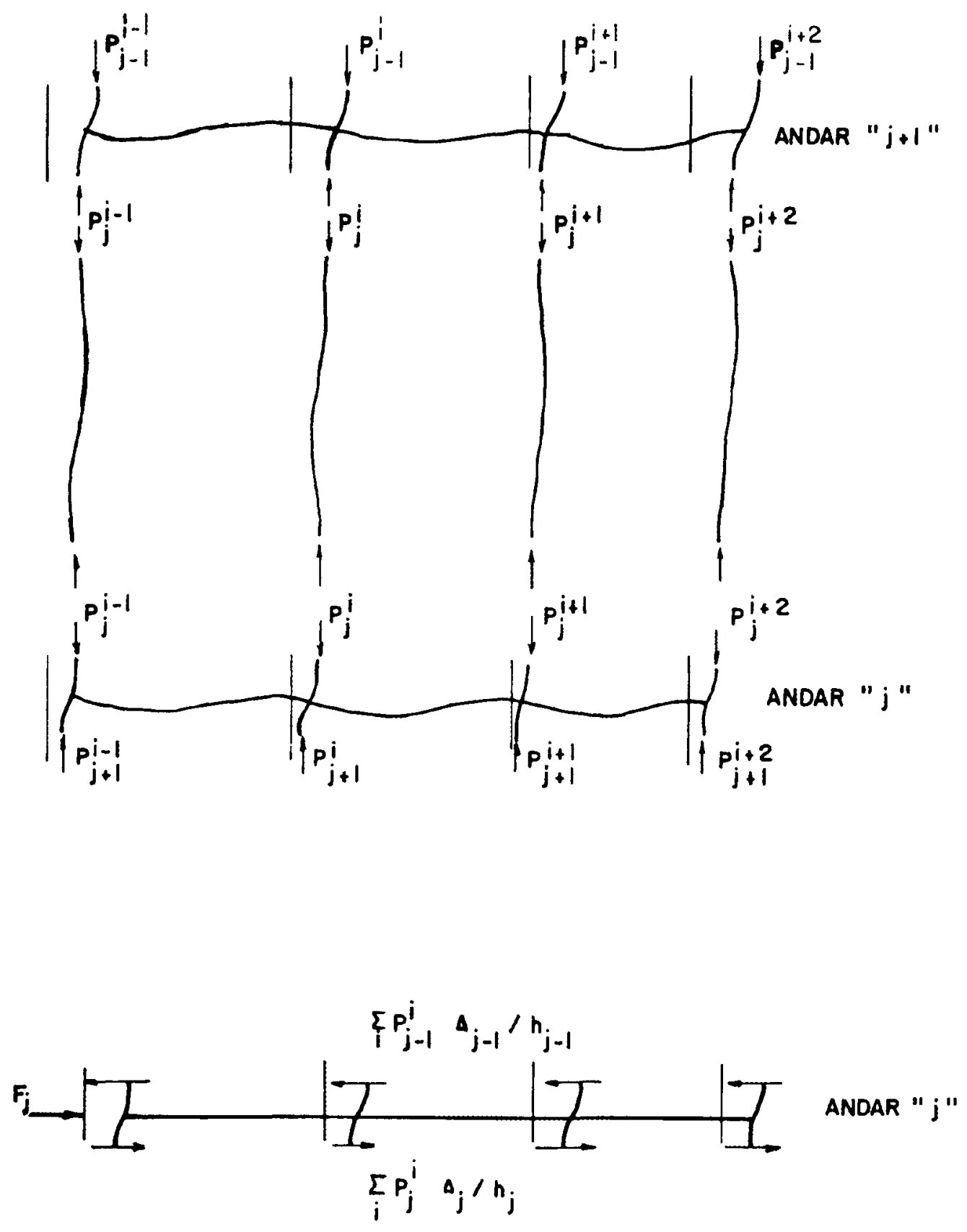

FIGURA 2.2 - Cálculo das forças laterais equivalentes ao efeito P- $\Delta$ Assim, a força lateral fictícia a ser aplicada em cada andar para simular o efeito $P-\Delta$ será:

$$
F_{1}^{j}=V_{1}^{j}-V_{1}^{j-1}
$$


como mostrado na Fig. 2.2. As forças laterais fictícias são adicionadas ao carregamento lateral aplicado no pórtico, $\left(\mathrm{H}_{\mathrm{j}}\right)$. Uma nova análise em $1^{\mathrm{a}}$ ordem é então efetuada, repetindo-se a operação até que os deslocamentos obtidos em duas iterações consecutivas coincidam, a menos de uma tolerância préestabelecida, que conforme MACGREGOR \& HAGE (1977) deve ser de 5\%. Em geral, para estruturas aporticadas de razoável rigidez, dois ou três ciclos de iteração são suficientes para a convergência, e os esforços e deslocamentos obtidos agora incluem o efeito P- $\Delta$. Deve-se salientar que o efeito P- $\Delta$ não afeta apenas os esforços nas colunas mas também os esforços nas vigas.

A aplicação do método $\mathrm{P}-\Delta$ iterativo não está limitado apenas ao caso de combinação de cargas verticais e horizontais; caso com carregamento apenas vertical pode também ser considerado. Em ambos os casos é exigida a determinação da configuração deformada e o cálculo das forças laterais fictícias equivalentes usando-se as eq. (2.1) e eq. (2.2). As forças laterais são então aplicadas à estrutura e a configuração deformada é novamente determinada. Se o carregamento é apenas vertical deslocamentos laterais significantes podem não ser encontrados. Nestes casos a análise deve ser feita com base numa rotação dos andares igual ao provável desaprumo tolerável (da ordem de 1/200 para pórticos de concreto).

\subsubsection{Análise P- $\Delta$ Direta}

Alternativamente é possivel obter uma equação para obtenção dos deslocamentos finais de $2^{\mathrm{a}}$ ordem. A força horizontal total aplicada no andar “j” é (Fig. 2.1.a):

$$
\mathbf{H}_{1}^{j}=\sum_{\mathbf{i}=1}^{\mathrm{j}} \mathbf{H}_{\mathbf{i}}
$$


Se $\mathrm{K}^{\mathrm{j}}$ é deslocamento lateral causado por uma força lateral unitária no andar “j”, então o deslocamento de $1^{a}$ ordem $\left(1^{\circ}\right.$ ciclo $)$ do andar j é:

$$
\Delta_{1}^{\mathrm{j}}=\mathrm{K}^{\mathrm{j}} \mathrm{H}_{1}^{\mathrm{j}}
$$

Para $02^{\circ}$ ciclo a força horizontal total no andar j é:

$$
\mathrm{H}_{2}^{\mathrm{j}}=\sum_{\mathrm{i}=1}^{\mathrm{j}}\left(\mathrm{H}_{\mathrm{i}}+\mathrm{F}_{1}^{\mathrm{i}}\right)
$$

onde $F_{1}^{i}$ é a força lateral para o andar i. Substituindo-se a eq. (2.2) na eq. (2.5) vem:

$$
\begin{aligned}
H_{2}^{j}= & \sum_{i=1}^{j}\left(H_{i}+\frac{\left(\sum P_{i}\right) \Delta_{1}^{i}}{h_{i}}-\frac{\left(\sum P_{i-1}\right) \Delta_{1}^{i-1}}{h_{i-1}}\right) \text { ou } \\
H_{2}^{j}= & \sum_{i=1}^{j} H_{i}+\frac{\left(\sum P_{1}\right) \Delta_{1}^{i}}{h_{1}}+\frac{\left(\sum P_{2}\right) \Delta_{1}^{2}}{h_{2}}-\frac{\left(\sum P_{1}\right) \Delta_{1}^{1}}{h_{1}} \\
& +\frac{\left(\sum P_{3}\right) \Delta_{1}^{3}}{h_{3}}-\frac{\left(\sum P_{2}\right) \Delta_{1}^{2}}{h_{2}}+\cdots+\frac{\left(\sum P_{j}\right) \Delta_{1}^{j}}{h_{j}} \\
& -\frac{\left(\sum P_{j-1}\right) \Delta_{1}^{j-1}}{h_{j-1}} \\
H_{2}^{j}= & \sum_{i=1}^{j} H_{i}+\frac{\left(\sum P_{j}\right) \Delta_{1}^{j}}{h_{j}}=H_{1}^{j}+V_{1}^{j}
\end{aligned}
$$

Assim

$$
\Delta_{2}^{\mathrm{j}}=\mathrm{K}^{\mathrm{j}} \mathrm{H}_{2}^{\mathrm{j}}=\mathrm{K}^{\mathrm{j}}\left(\mathrm{H}_{1}^{\mathrm{j}}+\frac{\left(\sum \mathrm{P}_{\mathrm{j}}\right) \Delta_{1}^{\mathrm{j}}}{\mathrm{h}_{\mathrm{j}}}\right)
$$




$$
\begin{aligned}
& \Delta_{2}^{j}=K^{j} H_{1}^{j}\left(\frac{\left(\sum P_{j}\right)\left(K^{j} \cdot H_{1}^{j}\right)}{h_{j}}\right) \\
& \Delta_{2}^{j}=K^{j} H_{1}^{j}\left(1+\frac{\left(\sum P_{j}\right) K^{j}}{h_{j}}\right)
\end{aligned}
$$

Para o 3ำ ciclo de iteração

$$
\mathrm{H}_{3}^{\mathrm{j}}=\mathrm{H}_{1}^{\mathrm{j}}+\mathrm{V}_{2}^{\mathrm{j}}
$$

Então, assumindo-se que as forças axiais permanecem, constantes, vem:

$$
\begin{aligned}
& \Delta_{3}^{j}=K^{j} H_{3}^{j}=K^{j}\left(H_{1}^{j}+\frac{\left(\sum P_{j}\right) \Delta_{2}^{j}}{h_{j}}\right) \\
& \Delta_{3}^{j}=K^{j}\left[H_{1}^{j}+\frac{\sum P_{j}}{h_{j}} K^{j} H_{1}^{j}\left(1+\frac{\sum P_{j} K^{j}}{h_{j}}\right)\right] \\
& \Delta_{3}^{j}=K^{j}\left[1+\frac{\left(\sum P_{j}\right) K^{j}}{h_{j}}+\left(\frac{\left(\sum P_{j}\right) K^{j}}{h_{j}}\right)^{2}\right]
\end{aligned}
$$

Entretanto para o enésimo ciclo, vem:

$$
\Delta_{n}^{j}=K^{j} \cdot H_{i}^{j}\left[1+\frac{\left(\sum P_{j}\right) K^{j}}{h_{j}}+\left(\frac{\left(\sum P_{j}\right) K^{j}}{h_{j}}\right)^{2}+\cdots+\left(\frac{\left(\sum P_{j}\right) K^{j}}{h_{j}}\right)^{n-1}\right]
$$


Se a série entre colchetes converge a estrutura se estabiliza; se ao contrário, a série diverge, a estrutura não pode encontrar uma posição de equilíbrio. Esta série é uma progressão geométrica de razão $\left[\left(\sum \mathrm{P}_{\mathrm{j}}\right) \mathrm{K}^{\mathrm{j}} / \mathrm{h}_{\mathrm{j}}\right]$, cuja expressão da soma desta série até o enésimo termo é:

$$
S_{n}=\frac{1-r^{n}}{1-r}
$$

onde

$$
\mathrm{r}=\frac{\left(\sum \mathrm{P}_{\mathrm{j}}\right) \mathrm{K}^{\mathrm{j}}}{\mathrm{h}_{\mathrm{j}}}
$$

Se $\mathbf{r}>1$ a série não converge.

$$
\text { Se } r<1 \Rightarrow \lim _{n \rightarrow \infty} r^{n}=0 \Rightarrow \lim _{n \rightarrow \infty} S_{n}=\frac{1}{1-r}
$$

aplicando-se a eq. (2.9), resulta que o deslocamento final é:

$$
\begin{aligned}
& \Delta_{\mathrm{f}}=\mathrm{K}^{\mathrm{j}} \mathrm{H}_{1}^{\mathrm{j}}\left(\frac{1}{1-\frac{\left(\sum \mathrm{P}_{\mathrm{j}}\right) \mathrm{K}^{\mathrm{j}}}{\mathrm{h}_{\mathrm{j}}}}\right) \\
& \Delta_{\mathrm{f}}=\Delta_{\mathrm{i}}^{\mathrm{j}}\left(\frac{1}{1-\frac{\left(\sum \mathrm{P}_{\mathrm{j}}\right) \mathrm{K}^{\mathrm{j}}}{\mathrm{h}_{\mathrm{j}}}}\right)
\end{aligned}
$$$$
\text { ou }
$$ 
A condição $r<1$ implica:

$$
\left(\sum \mathbf{P}_{\mathrm{j}}\right) \Delta_{1}<\mathrm{h}_{\mathrm{j}} \mathrm{H}_{1}^{\mathrm{j}}
$$

O termo

$$
\mathrm{Q}=\frac{\left(\sum \mathrm{P}_{\mathrm{j}}\right) \mathrm{K}^{\mathrm{j}}}{\mathrm{h}_{\mathrm{j}}}=\frac{\sum \mathrm{P}_{\mathrm{j}} \Delta_{1}^{\mathrm{j}}}{\mathrm{H}_{1}^{\mathrm{j}} \cdot \mathrm{h}_{\mathrm{j}}}
$$

é conhecido como parâmetro de estabilidade definido pelo ACI-89. O valor inverso é definido por HORNE (1975) como fator de carga crítica e dado por:

$$
\lambda_{\mathrm{c}}=\frac{\mathrm{H}_{1}^{\mathrm{j}} \mathrm{h}_{\mathrm{j}}}{\sum \mathrm{P}_{\mathrm{j}} \Delta_{\mathrm{l}}^{\mathrm{j}}}
$$

A equação (2.13) mostra que os deslocamentos de $2^{\mathrm{a}}$ ordem podem ser determinados diretamente de análise de $1^{\mathrm{a}}$ ordem se ocorre pouca ou nenhuma mudança na rigidez lateral do andar decorrente dos momentos de $2^{\mathrm{a}}$ ordem.

A partir da eq. (2.13) pode-se ter de forma aproximada o valor da carga crítica, que pode ser definida como a carga que anula o denominador da equação, levando-se os deslocamentos para o infinito. Assim uma aproximação da magnitude da carga crítica para o andar j é:

$$
P_{c}^{j}=\frac{H_{1}^{j} h_{j}}{\Delta_{1}^{j}}
$$

Uma expressão mais exata é dada por:

$$
P_{c}^{j}=\frac{H_{1}^{j} h_{j}}{\alpha \Delta_{1}^{j}}
$$


onde $\alpha$ varia de 1,0 para vigas flexíveis a 1,22 para vigas rígidas, conforme recomendação do ASCE (1978).

$O$ parâmetro de estabilidade $Q$ (eq. 2.14) pode ser usado para definir os pórticos como de nós fixos ou móveis, e a estabilidade do pórtico. Para o ACl 318-89 se $\mathrm{Q}<0,04$ o pórtico pode ser considerado com de nós fixos e os momentos de $2^{\mathrm{a}}$ ordem não excedem, em $5 \%$ os momentos de $1^{\mathrm{a}}$ ordem. MACGREGOR \& HAGE (1977) mostraram que 5\% de acréscimo nos deslocamentos deveria ser esperado se $Q<0,0475$; para $Q>0,22$ recomendam uma análise não-linear mais exata.

\subsubsection{Método $P-\Delta$ Amplificador de Momento}

Este método de análise é usualmente uma versão simplificada do método $P-\Delta$ direto discutido no ítem anterior.

Para a coluna mostrada na Fig. 2.3 o momento fletor máximo é dado por:

$$
\mathrm{M}_{\text {max }}=\mathrm{Hh}+\sum \mathrm{P} \Delta_{\text {max }}
$$

Da equação (2.13) e (2.14)

$$
\Delta_{\max }=\Delta_{1}\left(\frac{1}{1-\frac{\mathrm{P} \Delta_{1}}{\mathrm{Hh}}}\right)
$$

e conseqüentemente: 


$$
M_{\max }=H h+\sum \mathrm{P}\left(\frac{1}{1-\frac{\sum \mathrm{P} \Delta_{1}}{\mathrm{Hh}}}\right) \Delta_{1}
$$

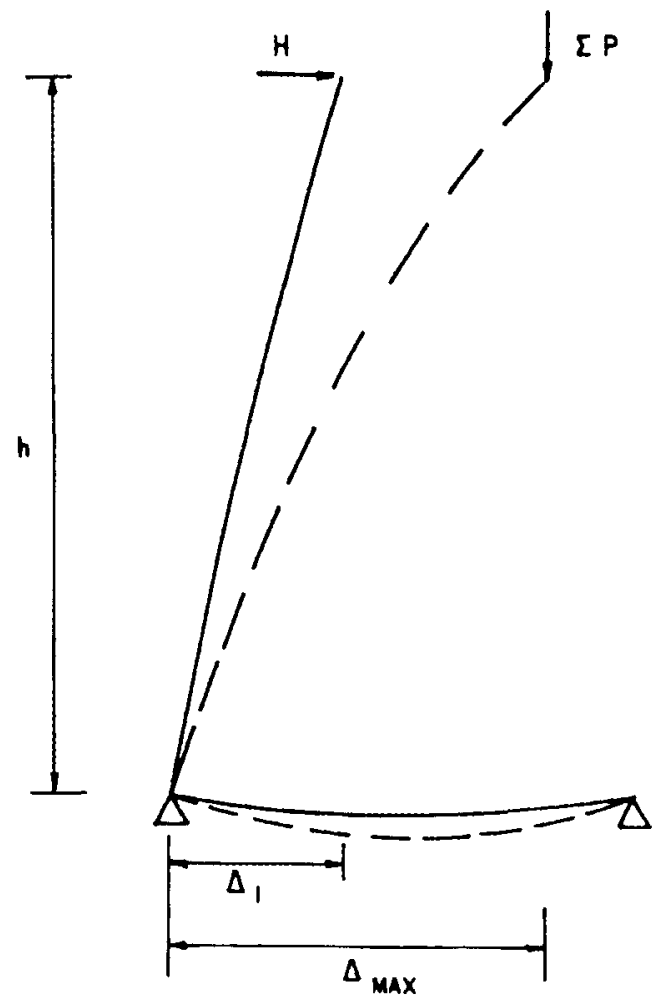

FIGURA 2.3 - Forças na coluna deformada

isto é, o mesmo que:

$$
M_{\max }=H h+\frac{\frac{\sum P \Delta_{1}}{H h} H h}{\left(1-\frac{\sum P \Delta_{1}}{H h}\right)} \quad \text { ou }
$$




$$
\begin{aligned}
& M_{\max }=H h+\left(\frac{\frac{\sum P \Delta_{1}}{H h}}{1-\frac{\sum P \Delta_{1}}{H h}}\right) H h \\
& M_{\max }=H h \frac{\left(1-\frac{\sum P \Delta_{1}}{H h}+\frac{\sum P \Delta_{1}}{H h}\right)}{\left(1-\frac{\sum P \Delta_{1}}{H h}\right)} \text { ou } \\
& M_{\max }=H h \frac{1}{\left(1-\frac{\sum P \Delta_{1}}{H h}\right)}
\end{aligned}
$$

ou em termos do parâmetro de estabilidade $Q$

$$
\begin{aligned}
& M_{\max }=H h\left(\frac{1}{1-Q}\right) \quad \text { ou } \\
& M_{\text {max }}=M_{1}\left(\frac{1}{1-Q}\right)
\end{aligned}
$$

Este método P- $\Delta$ amplificador de momentos é também conhecido como "método do parâmetro Q" porque ele usa o parâmetro de estabilidade "Q" para ampliar os momentos.

No manual de flambagem e estabilidade do CEB/FIP (1978) é proposto o fator de amplificação em termos de momentos. O método é desenvolvido para acelerar a avaliação das forças axiais e dos momentos, 
admitindo que para cada iteração as forças calculadas aumentam aproximadamente como uma série geométrica.

Se o momento obtido numa seção crítica no $1^{\circ}$ cálculo é $M_{1}$, o correspondente valor resultante da iteração seguinte é $\mathrm{M}_{2}$ e o momento da iésima iteração é $\mathrm{M}_{\mathrm{i}}$, então o fator geométrico "a" é dado por:

$$
a=\frac{M_{2}-M_{1}}{M_{1}}=\frac{M_{i}-M_{i-1}}{M_{i-1}-M_{i-2}}<1
$$

então, tem-se:

$$
\begin{aligned}
& M_{2}=M_{1}+a M_{1} \\
& M_{3}=M_{1}+a M_{1}+a^{2} M_{1} \\
& \vdots \\
& M_{n}=M_{1}+a M_{1}+\cdots+a^{n-1} M_{1}
\end{aligned}
$$

para um número infinito de iterações, obtém-se:

$$
M=M_{1}\left(1+\sum_{n=1}^{\infty} a^{n}\right)
$$

como mostrado anteriormente para $\mathbf{a}<1$, a convergência da série dá:

$$
M=M_{1} \frac{1}{1-a}=\frac{M_{1}}{1-\frac{M_{2}-M_{1}}{M_{1}}}
$$

mas da Fig. 2.3 tem:

$$
\mathrm{M}_{1}=\mathrm{Hh} \quad \text { e } \quad \mathrm{M}_{2}=\mathrm{Hh}+\sum \mathrm{P} \Delta_{1}
$$


substituindo-se $\mathrm{M}_{1}$ e $\mathrm{M}_{2}$ na eq. (2.26), chega-se a mesma eq. (2.21)

Cabe salientar que o valor de $a=\frac{M_{2}-M_{1}}{M_{1}}$ deve ser pequeno. $O$ uso de valores não pequenos pode levar a erros na avaliação do momento final de $2^{\mathrm{a}}$ ordem "M".

\subsection{Métodos Baseados no Comprimento Efetivo da Coluna}

$\mathrm{Na}$ impossibilidade de se usar um programa de análise não-linear fisica e geométrica como descrito no ítem 2.2, ou métodos aproximados de análise que considerem a interação entre o carregamento e os deslocamentos (Métodos $\mathrm{P}-\Delta$ ), permitem as normas que se utilize procedimentos aproximados de consideração das não-linearidades baseados nos resultados da análise linear.

Estes métodos são baseados no comprimento efetivo da coluna. A partir da determinação do comprimento efetivo de cada coluna da estrutura em função das condições de vinculação nas suas extremidades, obtém-se fatores de amplificação dos momentos (ACI-318/89) ou momentos adicionais de $2^{\mathrm{a}}$ ordem (CEB/FIP).

A Fig. 2.4 ilustra de forma simplificada os passos destes métodos aproximados aplicados a um pórtico plano. Inicialmente isola-se a coluna comprimida, levando-se em consideração se este pórtico é de nós fixos ou nós móveis (Fig. 2.4a). A seguir substitui-se a coluna já isolada (Fig. 2.4b) por uma coluna bi-rotulada padrão (Fig. 2.4c) de comprimento efetivo $\left(\mathrm{k} \ell_{\mathrm{u}}\right)$ e excentricidades equivalentes $\left(\mathrm{e}_{\mathrm{ol}}\right.$ e $\left.\mathrm{e}_{\mathrm{o} 2}\right)$, de modo que a capacidade de carga desta coluna bi-rotulada esteja bem próxima da capacidade da coluna isolada (Fig. 2.4b). O passo final deste procedimento simplificado consiste em simular os efeitos de esbeltez da coluna real nesta coluna padrão através da amplificação dos esforços internos na sua seção crítica (Fig. 2.4d). Portanto 
nestes métodos, o projeto da coluna levando-se em consideração os efeitos de $2^{\mathrm{a}}$ ordem, se reduz à verificação do equilíbrio da seção transversal (Fig. 2.4d).

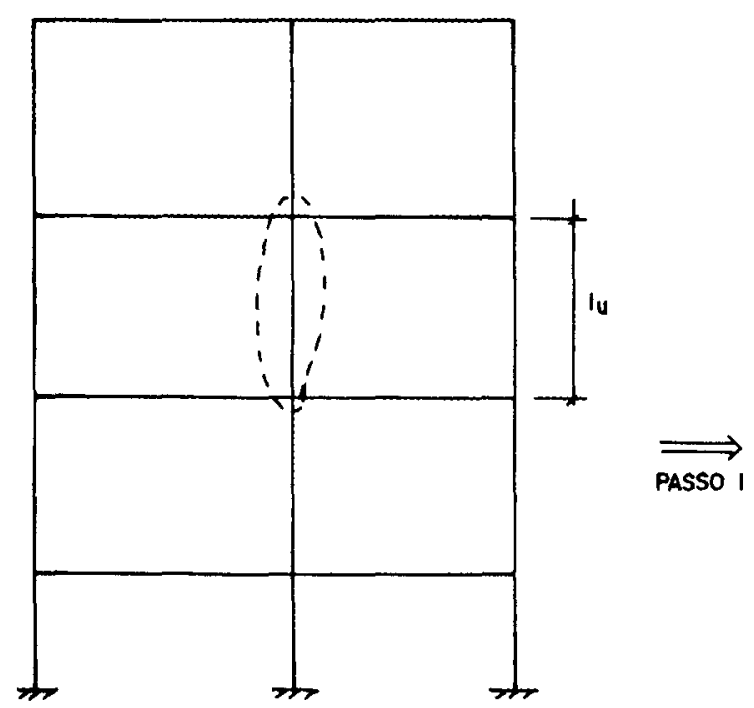

a) PÓRTICO

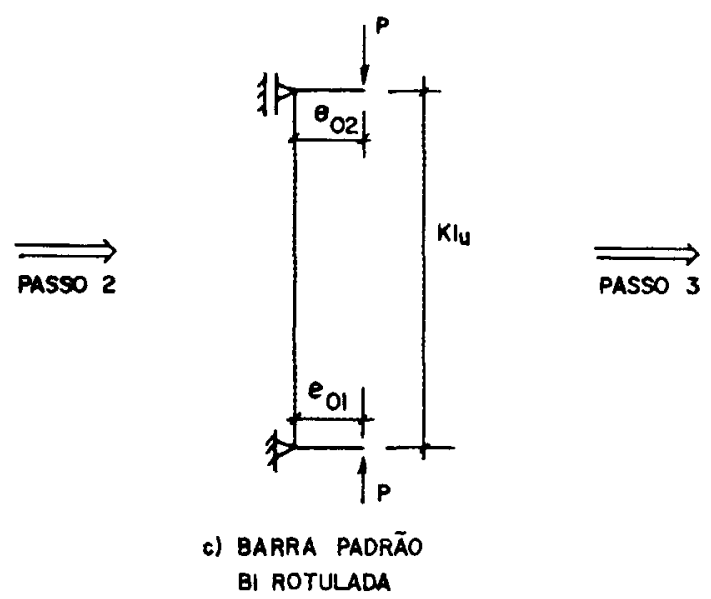

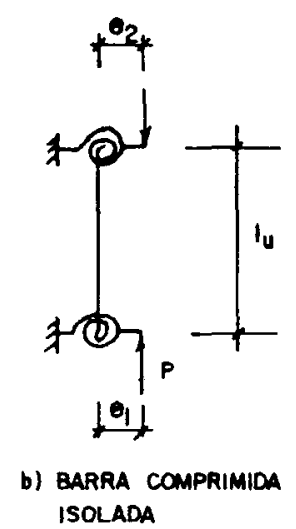

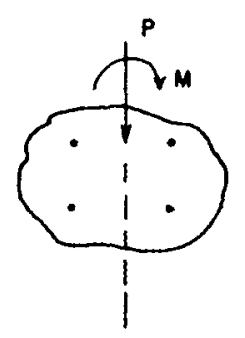

d) SEÇĀO TRANSVERSAL EM FLEXO-COMPRESSÃO

FIGURA 2.4 - Passos de um procedimento simplificado de projeto

\subsubsection{Comprimento Efetivo de Barras Compridas}

Como observado no ítem anterior os efeitos da esbeltez da coluna (efeitos de $2^{\mathrm{a}}$ ordem) podem ser levados em conta no projeto da coluna através do comprimento efetivo " $\mathrm{k} \ell \mathrm{u}$ ", da mesma forma que é feito nos projetos de colunas de aço conforme o AMERICAN INSTITUTE STEEL 
CONSTRUCTION (AISC). 0 coeficiente " $k$ " que multiplica o comprimento real da coluna " $\ell_{u}$ " é obtido com auxílio de um ábaco de dupla entrada, desenvolvido por Jackson e Moreland (Fig. 2.5), o qual permite a determinação gráfica deste fator para uma coluna de seção constante num pórtico de múltiplos andares e vários vãos.

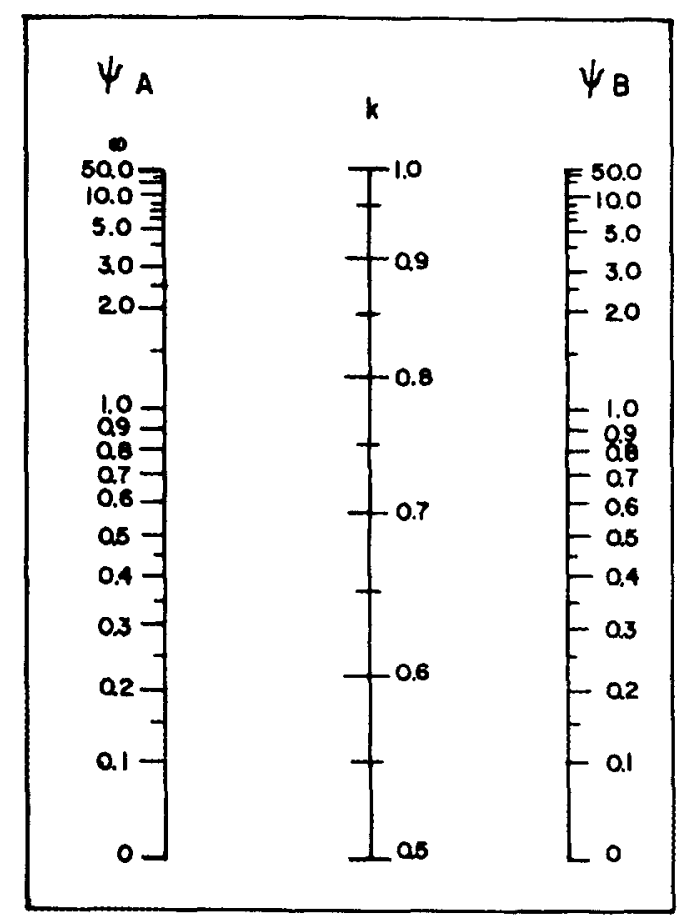

(a) nós fixos $\psi=\frac{\sum \mathrm{E}_{\mathrm{c}} \mathrm{I}_{\mathrm{c}} / \mathrm{L}_{\mathrm{c}}}{\sum \mathrm{E}_{\mathrm{v}} \mathrm{I}_{\mathrm{v}} / \mathrm{L}_{\mathrm{v}}}$

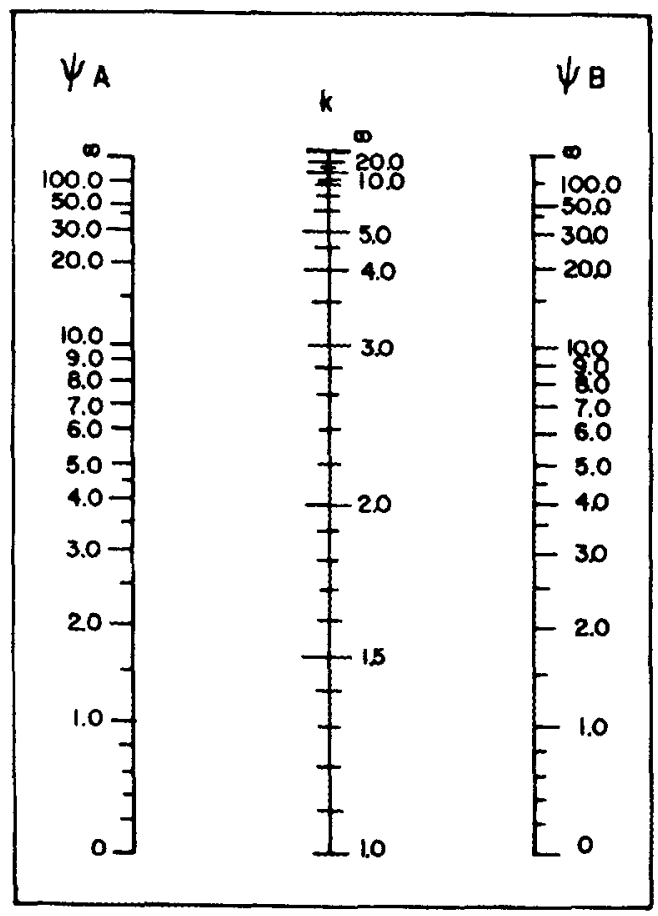

(b) nós móveis

FIGURA 2.5 - Fatores de comprimento efetivo

Devido à diferença de comportamento entre os pórticos de nós fixos e os de nós móveis, o fator de comprimento efetivo " $k$ " dependerá da deslocabilidade do pórtico. Na prática, raramente existirá um pórtico de nós completamente fixos ou completamente móveis. Para efeito de utilização dos ábacos da Fig. 2.5, considera-se que a coluna pertence a um pórtico de nós fixos se os deslocamentos horizontais não afetam significativamente os momentos na estrutura, o ACI-318/89 define o pórtico como de nós fixos, 
quando o parâmetro de estabilidade "Q", definido pela eq. (2.14) é menor que 0,04 .

Um procedimento alternativo, mais aproximado, pode ser usado para classificação dos pórticos em de nós fixos ou de nós móveis. Neste procedimento, a coluna pode ser considerada como pertencente a um pórtico de nós fixos se estiver localizada num andar no qual os elementos de contraventamento (paredes, treliças, núcleos) têm rigidez lateral total à forças laterais pelo menos 6 vezes a soma das rigidezes laterais de todas colunas do andar.

O comprimento efetivo é função da relação entre a rigidez das colunas e das vigas em cada extremidade da coluna, sendo assim ele será fortemente afetado pela perda de rigidez por fissuração e pela variação da rigidez de vigas e colunas em função da densidade de armadura. Para utilização do ábaco da Fig. 2.5, a rigidez à flexão de cada barra (EI) deve ser calculada baseado no momento de inércia da seção fissurada dada por:

$$
\begin{array}{ll}
\mathrm{EI}=0.5 \mathrm{E}_{\mathrm{c}} \mathrm{I}_{\mathrm{g}} & \text { (para as vigas) } \\
\mathrm{EI}=\mathrm{E}_{\mathrm{c}} \mathrm{I}_{\mathrm{g}}\left(0.2+1.2 \rho_{\mathrm{t}} \mathrm{E}_{\mathrm{s}} / \mathrm{E}_{\mathrm{c}}\right) & \text { (para as colunas) }
\end{array}
$$

onde:

$\mathrm{E}_{\mathrm{c}}$ é o módulo de elasticidade do concreto.

$I_{g}$ é o momento de inércia da seção plena da barra em relação ao seu eixo desprezando-se a contribuição da armadura.

$E_{s}$ é o módulo de elasticidade da armadura.

$\rho_{\mathrm{t}}$ é a razão entre a área total da armadura da coluna e a área da seção transversal. 
De acordo com o ACI-318/89, o fator de comprimento efetivo para colunas de pórticos de nós fixos e de nós móveis pode ser calculado pelas equações abaixo:

- para colunas de pórticos de nós fixos, o fator de comprimento efetivo será tomado como o menor dos valores obtidos das eqs. (2.29) e (2.30)

$$
\begin{aligned}
& \mathbf{k}=0.7+0.05\left(\psi_{\mathrm{A}}+\psi_{\mathrm{B}}\right) \leq 1.0 \\
& \mathrm{k}=0.85+0.05 \psi_{\text {min }} \leq 1.0
\end{aligned}
$$

onde $\psi_{\mathrm{A}}$ e $\psi_{\mathrm{B}}$ são os valores de $\psi$ obtidos da equação indicada na Fig. 2.5 para as duas extremidades da coluna e $\psi_{\min }$ é o menor dos dois valores.

- para colunas de pórticos de nós móveis com ambas extremidades da coluna restringidas, o fator de comprimento efetivo pode ser tomado como:

$$
\begin{array}{ll}
\mathbf{k}=\frac{20-\psi_{\mathrm{m}}}{20} \sqrt{1+\psi_{\mathrm{m}}} & \text { para } \quad \psi_{\mathrm{m}}<2 \\
\mathrm{k}=0.9 \sqrt{1+\psi_{\mathrm{m}}} & \text { para } \quad \psi_{\mathrm{m}} \geq 2
\end{array}
$$

onde $\psi_{\mathrm{m}}$ é a média de valores de $\psi$ para as duas extremidades.

- para colunas de pórticos de nós móveis com rótula em uma extremidade, tem-se:

$$
\mathbf{k}=2.0+0.3 \psi
$$

onde $\psi$ é valor para extremidade restringida 


\subsubsection{Considerações Sobre os Efeitos da Esbeltez}

$O$ índice de esbeltez de uma coluna é definido como a relação $\left(\mathrm{k} \ell_{\mathrm{u}}\right) / \mathrm{r}$ onde $r$ é o raio de giração da seção e dado por $r=\sqrt{\mathrm{I} / \mathrm{A}}$. Conforme o ACI$318 / 89$ os efeitos da esbeltez (efeitos de $2^{\mathrm{a}}$ ordem) podem ser desprezados para colunas pertencentes a pórticos de nós fixos desde que:

$$
\frac{\mathrm{k} \cdot \ell_{\mathrm{u}}}{\mathrm{r}}<34-12 \frac{\mathrm{M}_{1 \mathrm{~b}}}{\mathrm{M}_{2 \mathrm{~b}}}
$$

onde $\mathrm{M}_{1 \mathrm{~b}} / \mathrm{M}_{2 \mathrm{~b}}$ é a relação entre o menor e o maior momento fletor nas extremidades da coluna, sendo esta relação positiva se provoca curvatura simples e negativa se provoca curvatura reversa. Para colunas pertencentes a pórticos de nós móveis os efeitos de $2^{\mathrm{a}}$ ordem serão desprezados se $\left(\mathrm{k} \ell_{\mathrm{u}}\right) / \mathrm{r}<$ 22. Em ambos os casos assegura-se que pelo menos $95 \%$ da resistência da seção transversal será desenvolvida, sendo assim a capacidade de carga da coluna será governada pela sua resistência.

MACGREGOR et al (1970) baseado na análise de várias estruturas construídas concluiram que os efeitos de $2^{\mathrm{a}}$ ordem nas colunas poderiam ser desprezados em $90 \%$ das colunas de pórticos de nós fixos e em $40 \%$ das colunas de pórticos de nós móveis. $\mathrm{O}$ autor acredita que nas estruturas atuais, mais esbeltas, estas percentagens sejam bem menores.

O ACI-318/89 recomenda uma análise não-linear mais precisa para colunas com índice de esbeltez $\mathrm{k} \ell_{\mathrm{u}}>100$.

O CEB/FIP dispensa a consideração dos efeitos de $2^{\mathrm{a}}$ ordem nas colunas com índice de esbeltez menor que 25. Permite o uso de métodos aproximados de determinação dos efeitos de $2^{\mathrm{a}}$ ordem para colunas com índice 
de esbeltez menor que 40. Permite que se calcule a esbeltez das colunas de um andar de um pórtico de andares múltiplos de nós móveis através da expressão:

$$
\lambda=\sqrt{\frac{12 \mathrm{KA}}{\mathrm{h}}}
$$

onde

$\lambda$ é o índice de esbeltez das colunas de um andar.

$\mathrm{K}$ é o deslocamento horizontal relativo do andar em consideração em relação ao andar inferior, sob ação de uma carga unitária $\mathrm{H}=1$ aplicada no topo da estrutura a qual é considerada comportar-se linearmente, com um módulo de elasticidade $\mathrm{E}=1$.

A é a soma das áreas das seções transversais de todas as colunas do referido andar.

$h$ é a altura do andar

A norma brasileira NBR-6118 exige a consideração dos efeitos de $2^{\mathrm{a}}$ ordem nas colunas com índice de esbeltez maior que 40. Permite a utilização de métodos aproximados para colunas com índice de esbeltez entre 40 e 80 . Exige utilização de método exato que considere a relação momento curvatura baseado nos diagramas $\sigma-\varepsilon$ do concreto e do aço, ou por método aproximado devidamente justificado sempre que a esbeltez for superior a 80 .

Dentre os procedimentos aproximados para consideração dos efeitos de $2^{\mathrm{a}}$ ordem em barras comprimidas baseado no comprimento efetivo, destacase o Método do amplificador de momentos recomendado pelo ACI-318/89 e Método do momento adicional recomendado pelo CEB/FIP. 


\subsubsection{Método do Amplificador de Momentos}

Para colunas com índice de esbeltez, inferior a 100, pode-se determinar de forma aproximada os efeitos de $2^{\mathrm{a}}$ ordem baseado no conceito de um amplificador de momentos “ $\delta$ ". Este coeficiente amplia os momentos oriundos da análise em $1^{\mathrm{a}}$ ordem para computar os efeitos das forças axiais nestes momentos; e é função da relação entre a carga axial na coluna e sua carga crítica, da relação entre os momentos nas extremidades da coluna e da sua forma deformada.

O momento de cálculo ampliado pelos efeitos de $2^{\mathrm{a}}$ ordem será dado por:

$$
M_{c}=\delta_{b} M_{2 b}+\delta_{s} M_{2 s}
$$

sendo

$$
\begin{aligned}
& \delta_{\mathrm{b}}=\frac{\mathrm{C}_{\mathrm{m}}}{1-\frac{\mathrm{P}_{\mathrm{u}}}{\phi \mathrm{P}_{\mathrm{c}}}} \geq 1 \\
& \delta_{\mathrm{s}}=\frac{\mathrm{C}_{\mathrm{m}}}{1-\frac{\sum \mathrm{P}_{\mathrm{u}}}{\phi \sum \mathrm{P}_{\mathrm{c}}}} \geq 1 \\
& \mathrm{P}_{\mathrm{c}}=\frac{\pi^{2} \mathrm{EI}}{\left(\mathrm{K} \ell_{\mathrm{u}}\right)^{2}}
\end{aligned}
$$

onde

$\mathrm{P}_{\mathrm{u}}$ é a carga axial de cálculo na coluna obtida da análise em $1^{\mathrm{a}}$ ordem. 
$\Sigma \mathrm{P}_{\mathrm{u}}$ é a resultante das cargas axiais de cálculos de todas as colunas no andar.

$P_{c}$ é a carga crítica da coluna suposta bi-rotulada com comprimento efetivo " $k \ell_{\mathrm{u}}$ ".

$\mathrm{C}_{\mathrm{m}}$ é um fator de correção para levar em contar o fato do momento máximo amplificado não ocorrer nas extremidades.

$\phi$ é o fator de redução de resistência.

$\mathrm{M}_{2 \mathrm{~b}}$ é o momento de cálculo na coluna proveniente do carregamento vertical, em teoria de $1^{\text {a }}$ ordem.

$\mathrm{M}_{2 \mathrm{~s}}$ é o momento de cálculo na coluna proveniente do carregamento lateral, em teoria de $1^{\mathrm{a}}$ ordem.

No cálculo da carga crítica da coluna e na análise em teoria de $1^{\mathrm{a}}$ ordem para determinação dos esforços na coluna deve-se levar em consideração, pelo menos de forma aproximada, o efeito da fissuração na rigidez EI das barras. Recomenda-se usar as equações (2.27) e (2.28).

$O$ fator de correção $C_{m}$ para pórticos de nós móveis é igual a 1, e para pórticos de nós fixos é dado por:

$$
C_{m}=0.6+0.4 \frac{M_{1 b}}{M_{2 b}}
$$

onde $\mathrm{M}_{1 \mathrm{~b}} / \mathrm{M}_{2 b}$ é a relação entre o menor e o maior dos momentos fletores nas extremidades das barras, sendo esta relação positiva quando estes momentos provocam curvatura simples.

$O$ valor do comprimento efetivo " $\mathrm{k} \ell_{\mathrm{u}}$ " influencia no valor da carga crítica " $P_{c}$ ", assim os valores do amplificador de momentos " $\delta$ " serão influenciado pelo fato da coluna pertencer a um pórtico de nós fixos ou de nós móveis. Para pórticos de nós fixos, o amplificador " $\delta$ " usa fator de comprimento efetivo menor ou igual a 1 , enquanto que os pórticos de nós 
móveis este fator será maior que $1 . \mathrm{O}$ fator $\mathrm{C}_{\mathrm{m}}$ é igual a 1 para pórticos de nós móveis e entre 0,4 e 1 para pórticos de nós fixos. Assim, para pórticos de nós móveis a carga crítica " $\mathrm{P}_{\mathrm{c}}$ " será menor e o amplificador será maior.

Um sistema de piso de um edificio é suficientemente rígido no seu plano o que faz com que todas as colunas tenham a mesma deflexão lateral a menos que ocorra torção. Entretanto a força cortante em cada coluna será função de sua rigidez relativa à rigidez de todas colunas do pavimento. Assim num andar a coluna mais rígida tende a travar a coluna mais flexível. As Fig. 2.6 e 2.7 mostram esta interação de colunas para um pórtico. A Fig. 2.6 mostra que o fator de amplificação da coluna da esquerda é quase constante para uma dada carga. Por exemplo na Fig. 2.6 o fator de amplificação a ser aplicado na coluna da esquerda para $P_{1} / P_{c}=0,6$ e $P_{2}=0$ (ponto $B$ ), é quase igual ao fator de amplificação para $\mathrm{P}_{1} / \mathrm{P}_{\mathrm{c}}=0,3$ com $\mathrm{P}_{1}=\mathrm{P}_{2}$ (ponto $\mathrm{A}$ ), o qual representa a mesma carga total no pórtico.

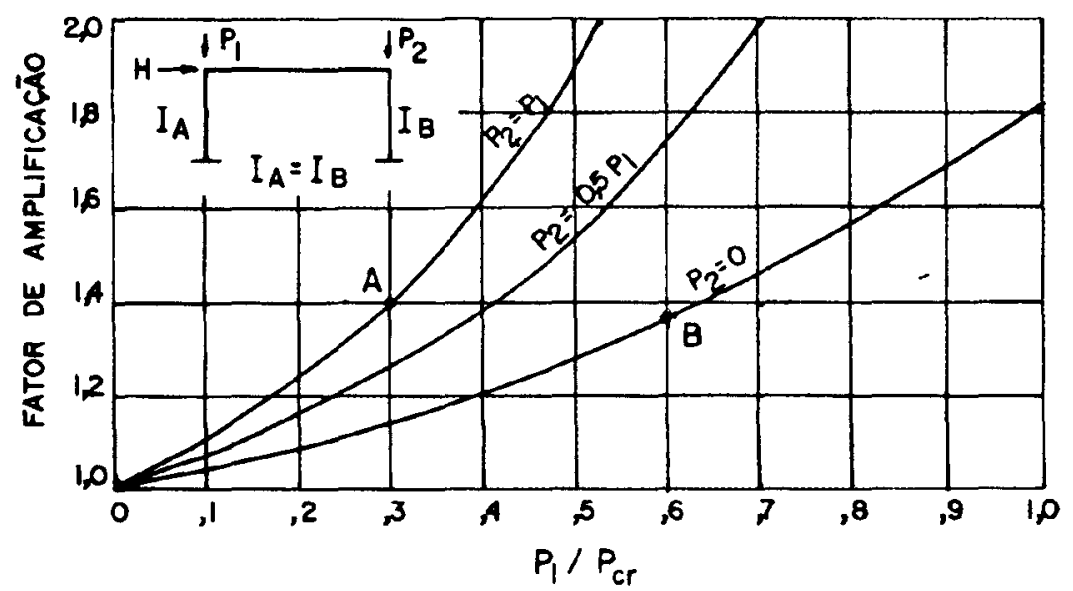

FIGURA 2.6 - Interação de colunas de mesma inércia de um pórtico birotulado 


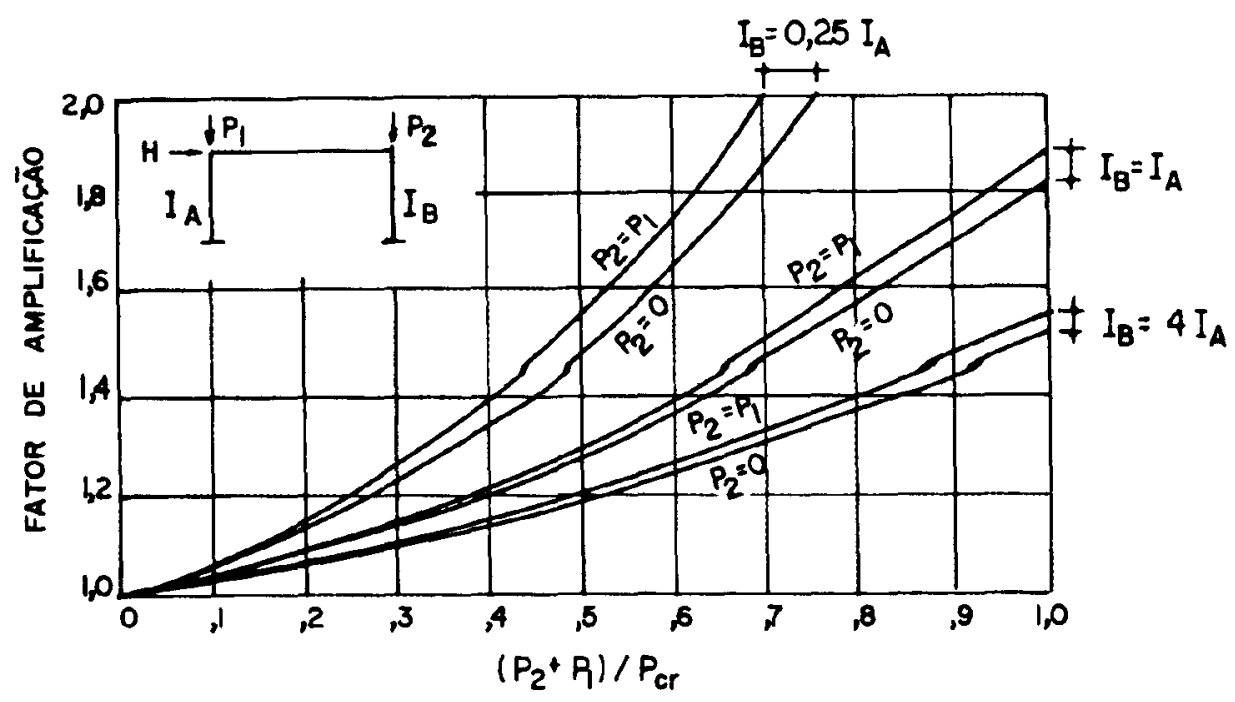

FIGURA 2.7 - Interação de colunas de inércia diferente um pórtico birotulado

Isto está mais evidente na Fig. 2.7, onde o fator de amplificação " $\delta$ " para a coluna da esquerda é plotado junto com os valores $\left(\mathrm{P}_{1}+\mathrm{P}_{2}\right) / \mathrm{P}_{\mathrm{c}}$. Para levar isto em consideração, o ACI-318/89 exige que o fator de amplificação comum a ser calculado para um andar seja baseado em $\Sigma \mathrm{P}_{\mathrm{u}} / \Sigma \mathrm{P}_{\mathrm{c}}$, onde $\Sigma$ representa a soma sobre todo o andar.

A resistência de um pórtico de nós móveis é governado pela estabilidade das colunas que é influenciada pelo grau de restrição aos deslocamentos impostos pelas vigas a elas ligadas. Se as ligações viga-coluna plastificam, conseqüentemente ocorrerá uma relaxação destas restrições e a capacidade de resistir cargas verticais do pórtico reduz drasticamente. Para evitar este problema o $\mathrm{ACI}-318 / 89$ recomenda que o projetista dimensione as vigas para resistir aos momentos amplificados das colunas, ou seja, as vigas devem ser capazes de absorver o momento total do nó.

YURA (1971) recomenda que para se prevenir contra a flambagem individual de uma coluna altamente carregada, é necessário verificar sua capacidade de carga usando sempre o fator do comprimento efetivo de pórtico de nós móveis.

As vantagens do método do amplificador de momentos são: 
- é simples e fácil de usar no dia a dia dos escritórios de projeto.

—é racional e apresenta resultados aceitáveis para a avaliação aproximada dos efeitos de $2^{\mathrm{a}}$ ordem.

— não é necessário conhecer os deslocamentos nodais.

As desvantagens do método são:

—é um método aproximado, onde os efeitos de $2^{\mathrm{a}}$ ordem são computados apenas nas colunas.

- os problemas inerentes ao uso de ábacos para determinação do comprimento efetivo, principalmente no tocante às condições de vinculação das colunas na fundação.

\subsubsection{Método do Momento Adicional}

Este método, recomendado pelo CEB/FIP e pela NBR -6118, é similar ao método do amplificador de momento descrito no ítem anterior, onde a carga axial de cálculo $P_{u}$ permanece inalterada. A diferença é que ao invés de multiplicar o momento de $1^{a}$ ordem da coluna pelo fator " $\delta$ ", adiciona-se a este momento de $1^{\mathrm{a}}$ ordem um momento de $2^{\mathrm{a}}$ ordem obtido do produto da força axial por uma excentricidade adicional, denominada excentricidade de $2^{\mathrm{a}}$ ordem.

O CEB/FIP recomenda que para coluna de seção transversal constante tendo excentricidades iguais com o mesmo sinal nas suas duas extremidades (Fig. 2.8), a excentricidade de $2^{\mathrm{a}}$ ordem a ser considerada seja:

$$
\mathrm{e}_{2}=\frac{\left(\mathrm{k} \ell_{\mathrm{u}}\right)^{2}}{10} \frac{1}{\mathrm{r}}
$$

onde $\mathrm{k} \ell_{\mathrm{u}}$ é o comprimento efetivo e $1 / \mathrm{r}$ é a curvatura da seção crítica. Para uma coluna de seção constante sujeita a momento de $1^{\text {a }}$ ordem variando linearmente ao longo do comprimento da coluna causando excentricidades de 
valores ou sinais diferentes (Fig. 2.9), a excentricidade de $2^{\mathrm{a}}$ ordem a ser considerada deve ser igual ao maior dos dois valores:

$$
\begin{aligned}
& e_{2}=0.6 e_{02}+0.4 e_{01} \\
& e_{2}=0.4 e_{02}
\end{aligned}
$$

onde $e_{01}$ e $e_{02}$ são as excentricidades de $1^{\mathrm{a}}$ ordem nas extremidades, $e_{02}$ sendo positiva e maior em módulo que $\mathrm{e}_{\mathrm{ol}}$.

Neste caso despreza-se os efeitos de $2^{\mathrm{a}}$ ordem se o índice de esbeltez é menor que $\left(50-25 \mathrm{e}_{\mathrm{o} 1} / \mathrm{e}_{02}\right)$.

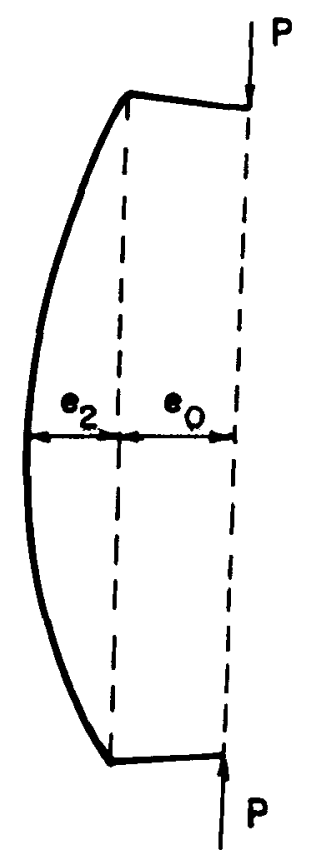

$$
\begin{aligned}
& e_{1}=e_{0}+e_{0} \\
& e_{0}=\text { EXCENT. DE } 1 \text { ORDEM } \\
& e_{0}=\text { EXCENT. ACIDENTAL } \\
& e_{1}=\text { EXCENT. DE } 2=\text { ORDEM }
\end{aligned}
$$

FIGURA 2.8 -Excentricidades iguais nas extremidades 

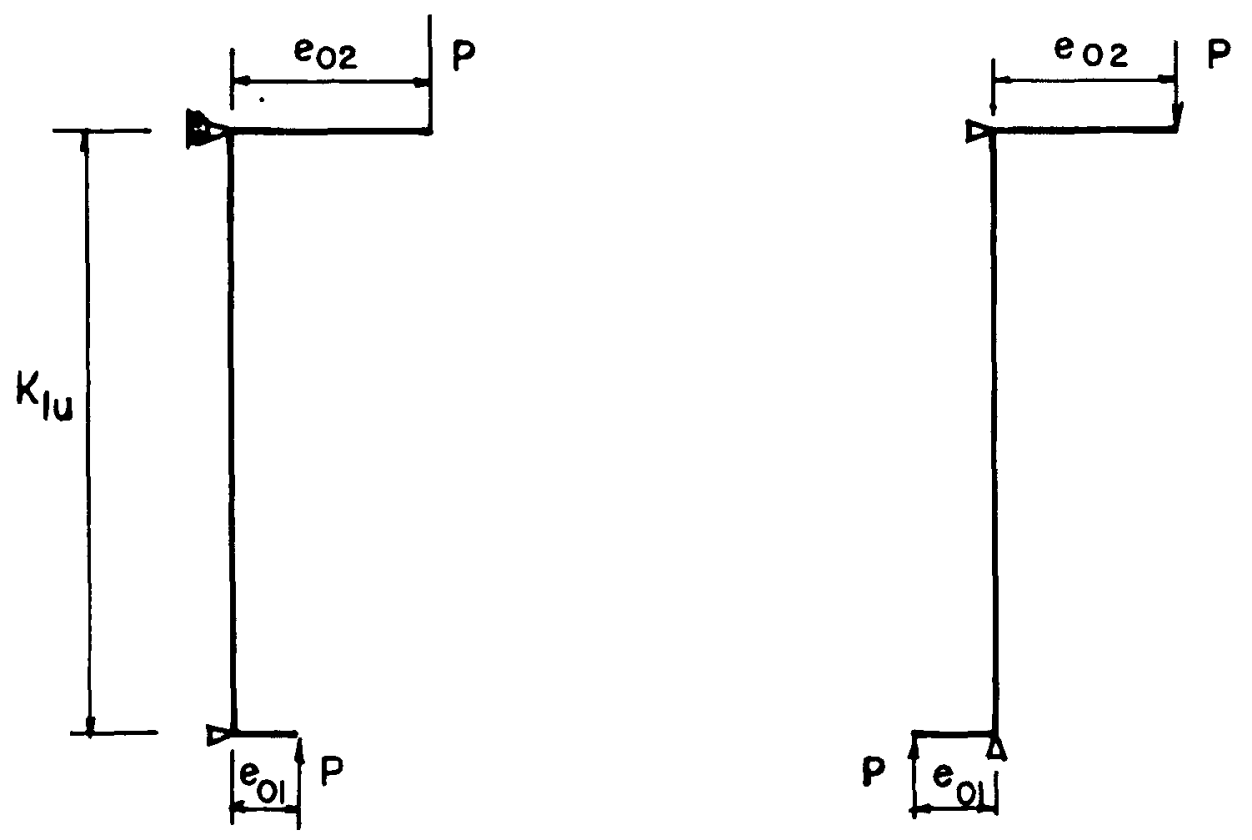

FIGURA 2.9 - Excentricidades diferentes nas extremidades

A NBR-6118 permite a consideração dos efeitos de $2^{\mathrm{a}}$ ordem de forma aproximada para índices de esbeltez entre 40 e 80, através da adição de um momento de $2^{\mathrm{a}}$ ordem. A expressão da excentricidade de $2^{\mathrm{a}}$ ordem é a mesma do CEB/FIP e é dada pela equação 2.41. A curvatura $1 / \mathrm{r}$ da seção crítica é dada por:

$$
\frac{1}{r}=\frac{0.0035+f_{y d} / E_{s}}{(v+0.5) h} \quad \text { com } \quad v+0.5 \geq 1
$$

sendo

$$
v=\frac{P_{u}}{A_{c} f_{c d}}
$$

onde

$\mathrm{h}=$ lado, paralelo à excentricidade acidental considerada, do retângulo mais estreito circunscrito à seção 
$\mathrm{P}_{\mathrm{u}}=$ força normal de cálculo

$\mathrm{A}_{\mathrm{c}}=$ área da seção transversal geométrica da coluna

$\mathrm{f}_{\mathrm{cd}}=$ resistência de cálculo do concreto à compressão

$\mathrm{f}_{\mathrm{yd}}=$ resistência de cálculo do aço

$\mathrm{E}_{\mathrm{s}}=$ módulo de elasticidade longitudinal do aço

Assim o momento adicional para computar os efeitos de $2^{\mathrm{a}}$ ordem na coluna será:

$$
M_{2 d}=P_{u} e_{2}
$$

As vantagens do método do momento adicional são:

- é simples e de fácil uso em escritórios de projetos,

- é racional e valores adequados para o projeto são obtidos em muitos casos.

As desvantagens são:

— os problemas inerentes à determinação correta do comprimento efetivo através de ábacos.

- é um método aproximado, onde os efeitos de $2^{\mathrm{a}}$ ordem são computados apenas nas colunas.

\subsection{Comentários Finais Sobre os Métodos Simplificados para Análise} Não-linear de Pórticos em Concreto Armado

Os métodos $\mathrm{P}-\Delta$ são mais racionais que os métodos de comprimento efetivo, e de simples utilização quando se pode usar o computador na análise. Quando não se dispõe de computador os métodos de comprimento efetivo são mais práticos pois não dependem dos valores dos deslocamentos nodais. No método P- $\Delta$ não há distinção entre pórticos de nós móveis ou de nós fixos, o 
que é a maior dificuldade nos métodos de comprimento efetivo uma vez que esta distinção é discutível.

O maior problema em qualquer análise não-linear simplificada de pórticos de concreto armado é a escolha adequada dos valores da rigidez à flexão EI, sob várias condições de carregamento. De fato, os erros resultantes do uso de métodos aproximados de calcular efeitos de $2^{\mathrm{a}}$ ordem ou carga crítica são, em geral, menores que os erros resultantes da escolha dos valores de EI.

Seria desejável que o valor de El refletisse a quantidade de armadura, a extensão da fissuração, o comportamento não-linear do concreto e do aço, os efeitos reológicos no concreto, a variação da rigidez ao longo da barra, mas isto ainda não é exeqüível no dia a dia dos projetistas que trabalham com sistemas estruturais de edifícios onde o grau de indeterminação estática é muito alto.

MACGREGOR \& HAGE (1977) têm recomendado trabalhar com EI igual a $0,4 E_{c} I_{g}$ nas vigas e $0,8 E_{c} I_{g}$ nas colunas, para computar os efeitos nãolineares. VASCONCELOS \& FRANCO (1991) sugerem considerar EI igual a $0,5 \mathrm{E}_{\mathrm{c}} \mathrm{I}_{\mathrm{g}}$ nas vigas e $0,8 \mathrm{E}_{\mathrm{c}} \mathrm{I}_{\mathrm{g}}$ nas colunas, ou alternativamente $0,7 \mathrm{E}_{\mathrm{c}} \mathrm{I}_{\mathrm{g}}$ para rigidez inicial da estrutura global.

MACGREGOR (1993) recomenda a introdução de um fator de redução da resistência $(\varphi)$ na análise em $2^{\mathrm{a}}$ ordem para levar em conta a variabilidade nas deflexões laterais previstas, resultante das simplificações na modelagem da estrutura e dos valores assumidos de E e I. Na proposta de revisão do ACI318/89 está incluída a recomendação de se utilizar na análise de pórticos os valores de EI propostos por MACGREGOR e HAGE (1977) multiplicados pelo fator de redução de resistência $\varphi=0.875$, resultando assim:

$$
\begin{aligned}
& -E I=0.35 E_{c} I_{g} \text { para vigas } \\
& -E I=0.70 E_{c} I_{g} \text { para colunas }
\end{aligned}
$$


- $\mathrm{EI}=0.70 \mathrm{E}_{\mathrm{c}} \mathrm{I}_{\mathrm{g}}$ para paredes não fissuradas

$-E I=0.35 \mathrm{E}_{\mathrm{c}} \mathrm{I}_{\mathrm{g}}$ para paredes fissuradas

$-E I=0.25 E_{c} I_{g}$ para lajes

onde $\mathrm{E}_{\mathrm{c}}$ é o módulo de elasticidade do concreto e $\mathrm{I}_{\mathrm{g}}$ é o momento de inércia da seção plena de concreto.

MACGREGOR (1993) comenta ainda que na proposta de revisão do ACI-318/89, o momento amplificado para pórticos de nós móveis será:

$$
\begin{aligned}
& M_{2}=M_{1 V}+\delta_{H} M_{1 H} \\
& \delta_{H}=\frac{1}{1-Q} \geq 1
\end{aligned}
$$

onde $\mathrm{M}_{2}$ é o momento amplificado incluindo-se o efeito de $2^{\mathrm{a}}$ ordem, $\mathrm{M}_{1 \mathrm{~V}}$ é $o$ momento de $1^{\mathrm{a}}$ ordem devido ao carregamento vertical, $\mathrm{M}_{1 \mathrm{H}}$ é o momento de $\mathrm{l}^{\mathrm{a}}$ ordem devido ao carregamento horizontal e $\mathrm{Q}$ é o parâmetro de estabilidade. 


\section{FORMULAÇÃO CONSISTENTE PARA ANÁlISE NÃO-LINEAR DE PÓRTICOS PLANOS}

\subsection{Introdução}

$\mathrm{Na}$ análise não-linear admite-se que a resposta da estrutura a um estado de carregamento não é linear, isto é, a incrementos iguais de cargas não correspondem necessariamente incrementos constantes de deslocamentos. Esta resposta não-linear está condicionada a mais de uma variável, caracterizandose o que o meio científico convencionou denominar não-linearidade física $\mathrm{e}$ geométrica de uma estrutura.

A não-linearidade geométrica está associada às variações de esforços e deslocamentos provocados pela mudança de geometria da estrutura sob ação do carregamento, seus efeitos na estrutura são comumente denominados "efeitos de segunda ordem".

Duas classes de problemas estão relacionados a uma análise com nãolinearidade geométrica: os que se prendem aos cálculos dos efeitos de segunda ordem numa estrutura sob ação de um determinado carregamento e os que se ligam diretamente aos fenômenos de perda de estabilidade que pode ocorrer por bifurcação do equilíbrio ou por aparecimento de ponto limite na configuração de equilíbrio.

A não-linearidade física está relacionada às características do comportamento mecânico dos materiais constituintes das estruturas. Este 
comportamento afeta a rigidez das seções transversais, tornando-as variáveis com o carregamento.

A teoria apresentada neste capítulo permite considerar 0 comportamento não-linear de estruturas de barras de material elasto-plástico, não coloca nenhuma restrição quanto à grandeza dos deslocamentos e das deformações, e aplica-se a problemas de análise em teoria de segunda ordem, assim como, aqueles relacionados à perda da estabilidade.

O desenvolvimento teórico será feito dentro da formulą̧ão Lagrangiana atualizada, utilizando-se um sistema de coordenadas naturais ou corrotacionais, obtendo-se assim de forma consistente as equações incrementais de equilíbrio, o vetor das forças nodais internas e a matriz de rigidez tangente de um elemento de pórtico plano.

\subsection{Revisão Bibliográfica}

O estudo do comportamento estrutural não-linear tem atraído muito a atenção dos pesquisadores. Sendo a análise não-linear um assunto muito amplo e tendo em vista os objetivos deste trabalho, a revisão bibliográfica aqui tratada será restrita a trabalhos publicados sobre análise não-linear de estruturas de barras, com ênfase nas formulações matriciais baseadas no Método dos Elementos Finitos.

O desenvolvimento do Método dos Elementos Finitos para a solução de problemas práticos de engenharia começou com o advento dos computadores e pela implementação da linguagem de programação FORTRAN, permitindo-se assim resolver problemas cada vez mais complexos e de crescente número de incógnitas.

Conforme BATHE (1996), as primeiras contribuições ao estudo do Método dos Elementos Finitos aplicado a engenharia aparecem nos trabalhos 
de ARGYRIS, J.H. \& KELSEY, S. (1955); TURNER, M.J. et al., (1956); e CLOUGH R.W. (1960). Mais tarde importantes foram as contribuições de ARGYRIS, J.H. (1965); e ZIENKIEWICZ, O.C. \& CHEUNG, Y.R. (1967).

$\mathrm{Na}$ década de 60 aparecem os primeiros trabalhos onde 0 comportamento não-linear geométrico (NLG) é formulado via o Método dos Elementos Finitos (MEF). CORRÊA (1991) cita o trabalho de TURNER et al. (1960) como o primeiro artigo publicado em que a não-linearidade geométrica é tratada pelo Método dos Elementos Finitos (MEF), usando um procedimento incremental linearizado, implementado junto ao conceito de rigidez tangente, usando relações deformação-deslocamento com termos de ordem superior. GALLAGHER \& PADLOG (1963) introduzem a não-linearidade física (NLF) no modelo de elementos finitos.

ARGYRIS (1965) estuda os problemas não-lineares físicos e geométricos (NLFG) pelo MEF, publicado no clássico texto "Contínua e Descontínua".

MALLET \& MARCAL (1968) desenvolvem o formalismo das matrizes incrementais incluindo significantes termos não obtidos anteriormente. As matrizes são definidas de forma explicita e apropriadas para deslocamentos finitos, grandes deslocamentos $\mathrm{e}$ análise de estabilidade. Usando sistemas de coordenadas Lagrangianas obtém as matrizes de rigidez para análise NLG através de três formulações diferentes, a saber, energia potencial, direta e linear incremental.

JENNING (1968) emprega as coordenadas eulerianas na formulação da matriz de rigidez tangente. POWELL (1969) também emprega as coordenadas eulerianas, separando os deslocamentos associados às deformações das barras e os deslocamentos de corpo rígido, permitindo assim utilizar a formulação para grandes rotações dos nós. 
MARTIN (1969) apresenta um minucioso estado da arte sobre a utilização do MEF na análise NLG, abordando a questão das deformações e tensões iniciais.

ORAN (1973a, 1973b) apresenta uma matriz de rigidez tangente consistente com a teoria convencional de viga-coluna para pórticos planos, utilizando coordenadas eulerianas. Posteriormente estendido a pórticos espaciais elásticos, baseado na hipótese de pequenas deformações mas grandes rotações, alertando sobre a não comutatividade das rotações no espaço, não podendo portanto ser tratadas como vetores.

No Brasil, MANTILLA (1974) desenvolveu um método iterativo para análise não-linear geométrica utilizando o conceito de funções de estabilidade para obtenção da matriz de rigidez. Usa um procedimento iterativo do tipo Newton-Raphson, e para levar em conta a teoria dos grandes deslocamentos, uma atualização de coordenadas é feita em cada etapa.

BATHE et al. (1975) apresentam as formulações Lagrangianas aplicadas à análise dinâmica e estática de grandes deformações utilizando o método dos elementos finitos. Essas formulações têm um fundamento geral e consistente e são capazes de modelar problemas com ambas as nãolinearidades.

ARGYRIS et al. (1979a) introduzem o conceito de graus de liberdade naturais na formulação Lagrangiana atualizada. Com este procedimento os deslocamentos cartesianos podem ser separados em deslocamentos de corpo rígido e deslocamentos associado às deformações dos elementos. ARGYRIS et al (1979b) estendem esta formulação consistente a pórticos espaciais, onde o problema da não comutatividade das grandes rotações é resolvido usando os ângulos de Euler-Rodrigues.

ANTUNES (1978) estuda a instabilidade da estrutura tridimensional de edifícios altos usando uma formulação para pequenos deslocamentos e pequenas deformações, desprezando-se os efeitos do bimomento. 
$\mathrm{Na}$ década de 80, cresce muito o número de trabalhos que apresentam formulações gerais para análise não-linear física e geométrica de estruturas de barras. $\mathrm{Na}$ linha da formulação Lagrangiana atualizada utilizando graus de liberdade naturais pode-se citar GATTASS \& ABEL (1987) onde são obtidas expressões explícitas das matrizes de rigidez de barras de pórtico. Ainda nesta linha destaque deve ser dado à contribuição dos trabalhos de PIMENTA (1986a, 1986b, 1986c, 1988, 1989a, 1989b) na formação do autor. No trabalho de PIMENTA (1986a) são discutidos vários aspectos da análise não-linear de pórticos planos compostos por qualquer material. A teoria apresentada permite considerar barras não-homogêneas de material elasto-plástico e não coloca nenhuma restrição quanto a grandeza dos deslocamentos. Os vetores de forças nodais internas e a matriz de rigidez são obtidas de forma consistente. CONCI \& GATTASS [1989] utilizam a formulação natural para obtenção de um elemento finito não-linear de barra de seção aberta de paredes finas incluindo os efeitos de torção e bimomento.

SILVA (1989) utiliza o método P- $\Delta$ para a avaliação dos efeitos da não-linearidade geométrica no comportamento estrutural de estruturas tridimensionais de edifícios altos com núcleos resistentes. LAVALL (1989) utiliza as funções de estabilidade para estudar a não-linearidade geométrica de pórticos planos metálicos.

MEEK et al (1990) estudaram o comportamento NLFG de pórticos espaciais, através de uma formulação simples e eficiente de elementos de vigas colunas. CORREIA (1991) desenvolve um sistema computacional para análise não-linear de estruturas tridimensionais de edifícios altos considerando a nãolinearidade geométrica das barras e a não-linearidade física das lajes de concreto armado. PIMENTA \& YOJO $(1992,1993)$ a partir das equações da mecânica dos sólidos deformáveis, de forma totalmente consistente usando formulações variacionais para a obtenção das equações de equilíbrio, desenvolvem um modelo de barra totalmente não-linear (NLFG) e 
geometricamente exato, sem nenhuma limitação quando à magnitude dos deslocamentos e das deformações, adequado para análise de pórticos espaciais. $O$ problema da não comutatividade das rotações finitas no espaço é resolvida com auxílio da fórmula de Euler-Rodrigues.

MORI (1992) estuda a não-linearidade geométrica de estruturas tridimensionais de edifícios altos com núcleos resistentes considerando os efeitos do bimomento.

Com relação aos procedimentos para solução de problemas nãolineares, destaca-se os trabalhos de CRISFIELD (1980), OWEN \& GOMES (1984), BATHE \& CIMENTO (1980) e PROENÇA (1989) onde é feita uma revisão dos algorítmos disponíveis para solução de problemas não-lineares via MEF, destacando o estudo comparativo da eficiência destes algorítmos.

\subsection{Considerações Sobre as Formulações para Análise Não-linear via MEF}

Existem basicamente duas formulações diferentes para descrever o movimento de um corpo sujeito a deslocamento finitos, que apesar de equivalentes, tem características específicas que as tornam aplicáveis à solução de determinados tipos de problemas. Na literatura elas são chamadas de Lagrangiana ou referencial e Euleriana ou espacial. A primeira é aplicável principalmente a problemas de mecânica dos sólidos e a última a problemas de mecânica dos fluidos.

PIMENTA (1989a) mostra que ambas as formulações podem ser utilizadas para obtenção da matriz de rigidez tangente de elementos finitos de volume pelo processo dos deslocamentos.

A principal característica da formulação Lagrangiana apresentada por BATHE et al (1975), para descrição do movimento de um corpo, é a utilização 
do tempo e da posição de um ponto do corpo em relação a uma configuração de referência como variáveis independentes. Quando se usa a configuração inicial como referência a formulação é dita Lagrangiana total (FLT). Caso a configuração de referência seja a imediatamente anterior a atual, a formulação é dita Lagrangiana atualizada (FLA).

A formulação Lagrangiana atualizada apresenta maior simplicidade que a FLT para a análise de problemas de grandes deslocamentos e pequenas deformações. A FLT possibilita considerar de forma mais direta leis constitutivas que dependem da história do carregamento, é fácil de implementar, com os deslocamentos sendo calculados diretamente. Contudo a principal desvantagem é não ser possível distinguir o deslocamento de corpo rígido do elemento das suas deformações locais, o que pode levar a uma descrição errada do equilíbrio, no caso de problemas de grandes deslocamentos. Na FLA é possível separar os deslocamentos de corpo rígido do elemento das suas deformações locais, mas embora descreva de forma mais exata o campo de deslocamentos, o trabalho de cálculo das deformações locais dos elementos é grande. Essa formulação é recomendada para problemas onde se tenha de considerar grandes deslocamentos, os quais requerem o uso da formulação atualizada para se obter resultados mais precisos. BATHE \& BOULOURCHI (1979) utilizam a FLA aplicada a elementos de vigas, e mostram que a FLA e a FLT levam a idênticos elementos de rigidez e vetores de forças nodais e que a FLA é computacionalmente mais efetiva.

Uma variante desta formulação FLA é a chamada formulação Lagrangiana parcialmente atualizada (FLPA), na qual a configuração de referência é a do passo de carga imediatamente anterior, diferindo da FLA pelo fato das coordenadas de cada elemento serem atualizadas apenas no início de cada passo de carga, com os cálculos dentro do passo conduzidos como uma formulação total (FLT). Esta formulação reúne, então, as vantagens da FLT (simplicidade) e da FLA (exatidão), exige entretanto que haja uma 
proximidade entre a configuração de uma iteração e a outra, pois se assume que a diferença entre ambas seja tão pequena que elas possam ser confundidas, exigindo o uso de elementos finitos de pequeno tamanho. Esta formulação que foi utilizada por WEN \& RAHIMZADEH (1983) também será utilizada neste trabalho.

Conforme dito anteriormente a formulação Euleriana é usualmente utilizada na análise de problemas de mecânica dos fluídos, na qual a atenção é focada no movimento material através de um controle estacionário de volume. Ao usar esta formulação em problemas estruturais deve se trabalhar com campo de velocidade, taxas de deformação obtidos através de relações da mecânica dos sólidos como funções lineares das velocidades nodais. Esta formulação exige portanto uma consistente e estável integração no tempo como destaca PIMENTA (1989a).

\subsection{Considerações Sobre os Sistemas de Coordenadas}

Existem basicamente dois sistemas de coordenadas a escolher para a obtenção da matriz de rigidez do elemento: coordenadas corrotacionais $\mathrm{e}$ coordenadas cartesianas ou Lagrangianas.

No sistema de coordenadas corrotacional os graus de liberdade ou coordenadas-deslocamento são definidos tomando como referência a corda da barra deformada e são chamadas coordenadas-deslocamento naturais. No sistema de coordenadas cartesianas estes graus de liberdade são definidos em relação a corda da barra indeformada são chamados coordenadasdeslocamento cartesianas ou Lagrangianas.

Na Fig. 3.1 são mostradas as configurações indeformada (AB) e deformada $\left(\mathrm{A}^{*} \mathrm{~B}^{*}\right)$ de uma barra de pórtico plano, onde $\mathrm{X}$ e $\mathrm{Y}$ são os eixos cartesianos no sistema global estacionário de referência, $\mathrm{x}$ e y são os eixos 
cartesianos no sistema local da barra na configuração inicial indeformada $(\mathrm{AB})$ e $\mathrm{x}^{*}$ (corda da barra deformada) 0 eixo de referência do sistema de coordenadas corrotacional.

As coordenadas-deslocamento generalizadas no sistema global são $\mathrm{p}_{1}$ a $\mathrm{P}_{6}$, medidas no sistema fixo $X Y$,

As coordenadas-deslocamento naturais no sistema local da barra na posição deformada são $q_{1}, q_{2}$ e $q_{3}$, medidas no sistema móvel $x^{*}$, sendo que $q_{1}$ mede a variação do comprimento da corda, $\mathrm{q}_{2}$ e $\mathrm{q}_{3}$ medem as rotações das extremidades da barra, independentemente da rotação de corpo rígido $\theta_{0}$, então:

$$
\begin{aligned}
& \mathrm{q}_{1}=\Delta=\ell_{\mathrm{c}}-\ell_{\mathrm{r}} \\
& \mathrm{q}_{2}=\rho_{1} \\
& \mathrm{q}_{3}=\rho_{2}
\end{aligned}
$$

As coordenadas-deslocamento cartesianas no sistema local x,y são $\left\{\mathrm{u}_{1}, \mathrm{v}_{1}, \theta_{1}, \mathrm{u}_{2}, \mathrm{v}_{2}, \theta_{2}\right\}$

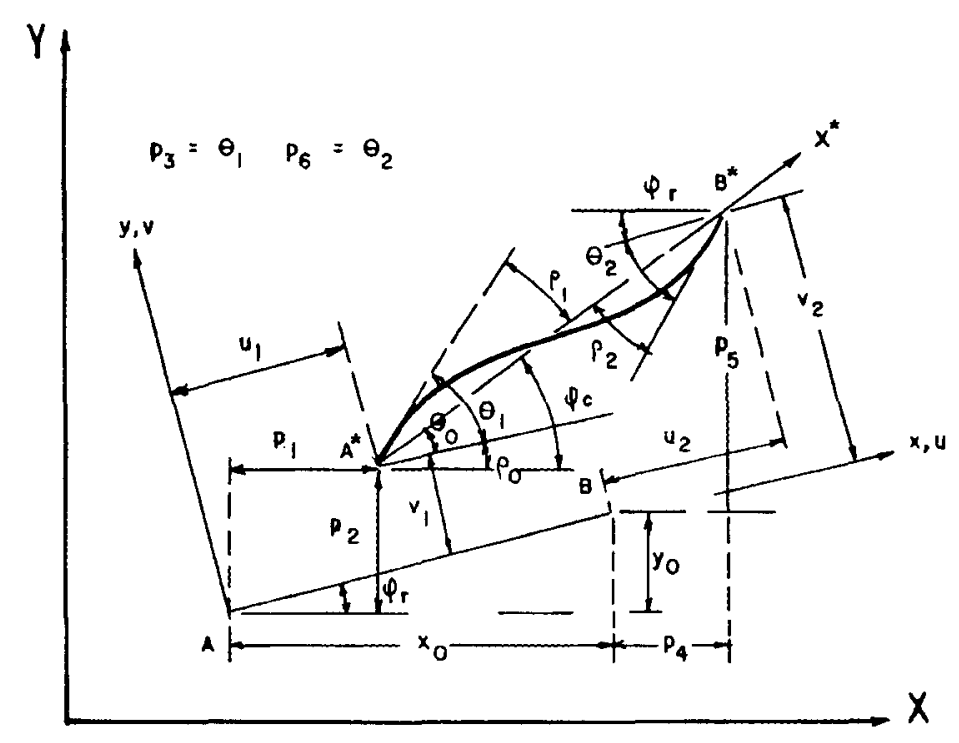

FIGURA 3.1 - Coordenadas-deslocamento de um elemento de pórtico plano nos sistemas cartesianos e corrotacional 
Da figura 3.1 vem:

$$
\begin{aligned}
& \Delta=\left[\left(x_{0}+p_{4}-p_{1}\right)^{2}+\left(y_{0}+p_{5}-p_{2}\right)^{2}\right]^{1 / 2}-\ell_{r} \\
& \rho_{1}=\varphi_{r}+p_{3}-\varphi_{c}=\theta_{1}-\theta_{0} \\
& \rho_{2}=\varphi_{r}+p_{6}-\varphi_{c}=\theta_{2}-\theta_{0}
\end{aligned}
$$

onde $\varphi_{\mathrm{r}}$ é a inclinação da barra $\mathrm{AB}$ em relação ao eixo $\mathrm{x}, \theta_{0}$ é o giro da corda, $\theta_{1}$ e $\theta_{2}$ são os giros de nó, $x_{0}$ e yo são as projeções da barra $A B$ sobre os eixos $\mathrm{X} \mathrm{e} \mathrm{Y,} \mathrm{respectivamente,} \ell_{\mathrm{r}}$ é o comprimento inicial da barra indeformada e $\varphi_{\mathrm{c}}$ é a inclinação da corda da barra deformada em relação ao eixo $X$, estes últimos dados por:

$$
\begin{aligned}
& \ell_{r}=\left(x_{o}^{2}+y_{0}^{2}\right)^{1 / 2} \\
& \varphi_{c}=\operatorname{arctg}\left[\frac{y_{0}+p_{5}-p_{2}}{x_{o}+p_{4}-p_{1}}\right]
\end{aligned}
$$

Usando funções de forma apropriadas para interpolação dos deslocamentos em função dos parâmetros nodais em coordenadas naturais $\left(\mathrm{q}_{1}\right.$, $q_{2}$ e $q_{3}$ ) e relações deslocamento-deformação, pode-se expressar a deformação longitudinal $\varepsilon$ e conseqüentemente a energia de deformação $U$ em função destas coordenadas-deslocamento. A partir daí obter-se a matriz de rigidez do elemento nesta coordenadas. Cabe ressaltar que estes graus de liberdade naturais são grandezas objetivas, isto é, independentes do movimento de corpo rígido. 
Analogamente é possível usando funções de forma e relações deformação-deslocamento numa formulação matricial do MEF, obter-se a matriz de rigidez do elemento, adotando-se o sistema de coordenadas locais cartesiano.

\section{5 . Considerações Sobre as Deformações e as Tensões}

Seja uma fibra de material onde se designa por $A_{\mathrm{r}}, \ell_{\mathrm{r}}, \mathrm{V}_{\mathrm{r}}$ a sua área da seção transversal, o seu comprimento e o seu volume, respectivamente, numa dada configuração de referência ou inicial; por $A_{c}, \ell_{c}, V_{c}$ a sua área da seção transversal, o seu comprimento e o seu volume, respectivamente, na configuração deformada ou atual. Nesta fibra age uma força normal $\mathrm{N}$, conforme mostrado na Fig. 3.2

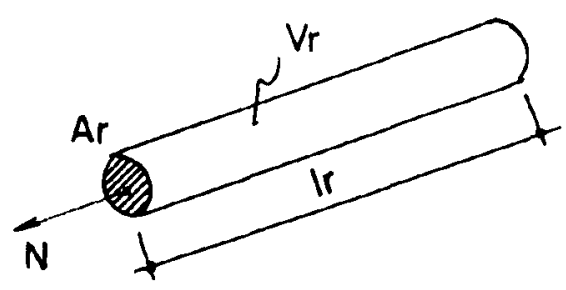

(a) CONFIGURACÃ̃O DE REFERẼNCIA

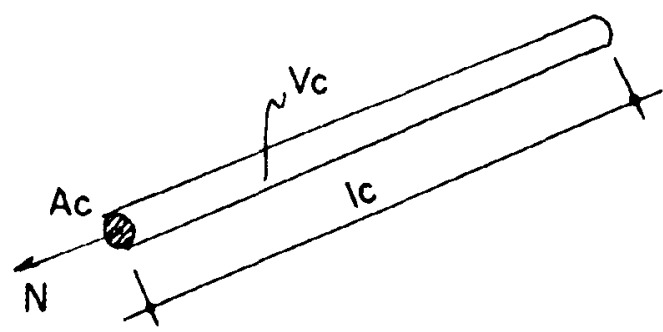

(b) CONFIGURAÇĀO ATUAL

FIGURA 3.2 - Configurações de uma Fibra de Material

Define-se como medida de deformação da fibra qualquer grandeza que compare os comprimentos desta fibra nas configurações de referência e atual. Uma medida básica de deformação é o estiramento da fibra dado por:

$$
\lambda=\frac{\ell_{\mathrm{c}}}{\ell_{\mathrm{r}}}
$$


Conforme PIMENTA (1986a) baseado no estiramento $\lambda$ pode-se definir uma familia de medidas de deformação através de:

$$
\begin{array}{ll}
\varepsilon_{m}=\frac{\lambda^{2 m}-1}{2 m} & \text { para } m \neq 0 \\
\varepsilon_{m}=\ln \lambda & \text { para } m=0
\end{array}
$$

Desta família pode-se destacar alguns membros famosos como:

a) Deformação quadrática ou de Green: $\mathrm{m}=1$

$$
\varepsilon_{1}=\frac{\lambda^{2}-1}{2}
$$

explicitando em função dos comprimentos, com auxílio da eq. 3.9 vem:

$$
\varepsilon_{1}=\frac{1}{2}\left[\left(\frac{\ell_{\mathrm{c}}}{\ell_{\mathrm{r}}}\right)^{2}-1\right]=\frac{1}{2}\left(\frac{\ell_{\mathrm{c}}^{2}-\ell_{\mathrm{r}}^{2}}{\ell_{\mathrm{r}}^{2}}\right)
$$

Seja uma fibra genérica distante y do eixo da barra cujo comprimento infinitesimal antes da deformação é dx. Após a deformação esta fibra tem comprimento infinitesimal ds (Fig. 3.3) dado por:

$$
d s=\sqrt{(d x+d u)^{2}+d v^{2}}
$$

O estiramento $\lambda$ da fibra é dado por 


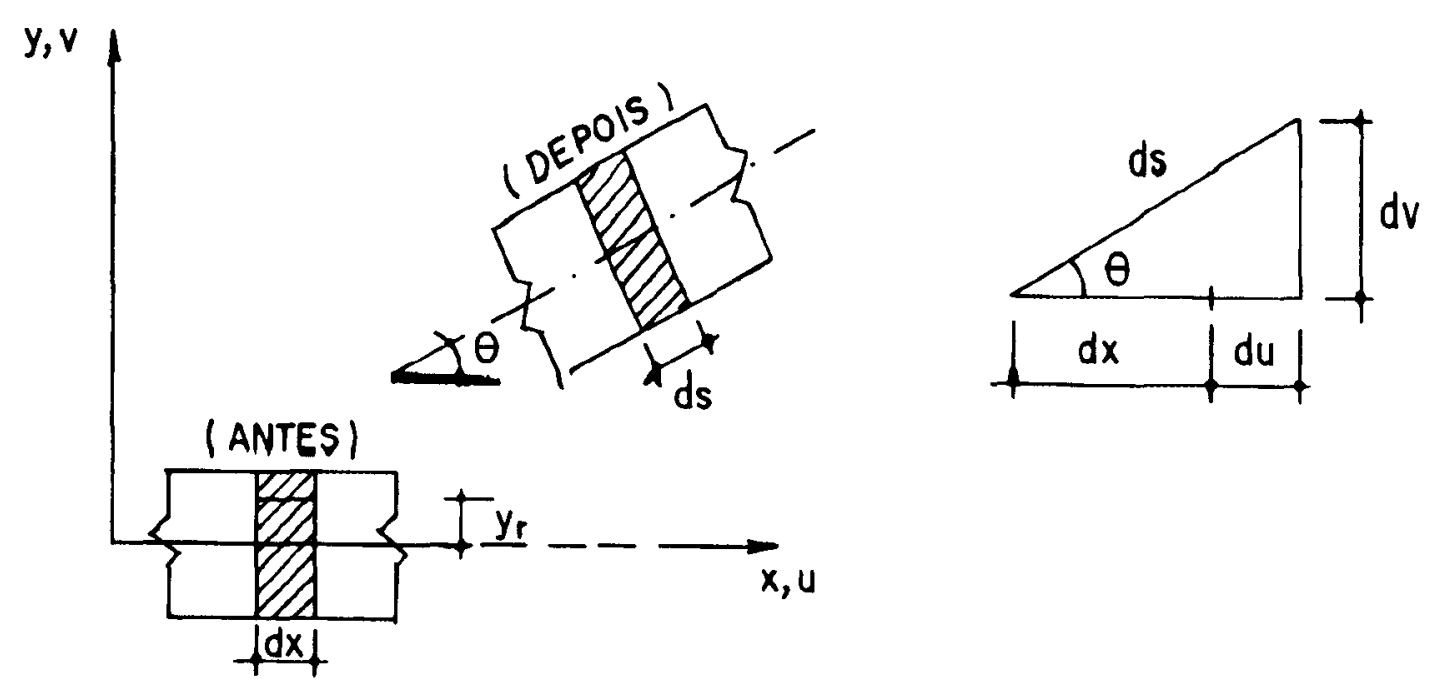

FIGURA 3.3 - Deformação de uma fibra

$$
\lambda=\frac{d s}{d x}=\frac{\sqrt{(d x+d u)^{2}+d v^{2}}}{d x}=\sqrt{\left(\frac{d x+d u}{d x}\right)^{2}+\left(\frac{d v}{d x}\right)^{2}}
$$

$\log 0$

$$
\begin{aligned}
& \lambda^{2}=\left(1+\frac{d u}{d x}\right)^{2}+\left(\frac{d v}{d x}\right)^{2} \quad \text { ou } \\
& \lambda^{2}=1+2 \frac{d u}{d x}+\left(\frac{d u}{d x}\right)^{2}+\left(\frac{d v}{d x}\right)^{2} \quad \text { ou } \\
& \frac{\lambda^{2}-1}{2}=\frac{d u}{d x}+\frac{1}{2}\left[\left(\frac{d u}{d x}\right)^{2}+\left(\frac{d v}{d x}\right)^{2}\right] \\
& \varepsilon_{1}=\frac{d u}{d x}+\frac{1}{2}\left[\left(\frac{d u}{d x}\right)^{2}+\left(\frac{d v}{d x}\right)^{2}\right]
\end{aligned}
$$

que é a expressão da deformação quadrática de Green, usual da Teoria da Elasticidade Não-linear. 
b) Deformação linear ou de engenharia $(m=1 / 2)$

$$
\varepsilon_{1 / 2}=\lambda-1
$$

explicitando em função dos comprimentos vem:

$$
\varepsilon_{1 / 2}=\frac{\ell_{\mathrm{c}}}{\ell_{\mathrm{r}}}-1=\frac{\ell_{\mathrm{c}}-\ell_{\mathrm{r}}}{\ell_{\mathrm{r}}}=\frac{\Delta \ell}{\ell_{\mathrm{r}}}
$$

Da figura 3.3 tem-se que:

$$
\begin{aligned}
& \lambda=\sqrt{\left(1+\frac{d u}{d x}\right)^{2}+\left(\frac{d v}{d x}\right)^{2}}, \log 0: \\
& \varepsilon_{1 / 2}=\sqrt{1+2 \frac{d u}{d x}+\left(\frac{d u}{d x}\right)^{2}+\left(\frac{d v}{d x}\right)^{2}}-1
\end{aligned}
$$

Portanto a deformação linear também tem termos não-lineares em $\frac{\mathrm{du}}{\mathrm{dx}}$ $\mathrm{e} \frac{\mathrm{dv}}{\mathrm{dx}}$

c) Deformação natural ou logarítmica ou de Hencky $(m=0)$

$$
\varepsilon_{0}=\ln \lambda=\ln \frac{\ell_{\mathrm{c}}}{\ell_{\mathrm{r}}}
$$

d) Deformação hiperbólica ou de Reiner (m = -1/2) 


$$
\begin{aligned}
& \varepsilon_{-\frac{1}{2}}=1-\lambda^{-1}=1-\frac{\ell_{\mathrm{r}}}{\ell_{\mathrm{c}}}=\frac{\ell_{\mathrm{c}}-\ell_{\mathrm{r}}}{\ell_{\mathrm{c}}} \text { ou } \\
& \varepsilon_{-\frac{1}{2}}=\frac{\Delta \ell}{\ell_{\mathrm{c}}}
\end{aligned}
$$

e) Deformação de Almansi: $m=-1$

$$
\begin{aligned}
& \varepsilon_{-1}=\frac{1-\lambda^{-2}}{2}=\frac{1}{2}\left(1-\frac{1}{\lambda^{2}}\right) \quad \text { ou } \\
& \varepsilon_{-1}=\frac{\ell_{c}^{2}-\ell_{r}^{2}}{2 \ell_{c}^{2}}
\end{aligned}
$$

Das equações 3.11 a 3.16 pode-se concluir que para pequenos alongamentos $(\lambda \cong 1)$ todos os membros da família de deformação se confundem.

Uma familia de taxas de deformação pode ser obtida derivando-se a eq. (3.10a) no tempo:

$$
\begin{aligned}
& \dot{\varepsilon}_{\mathrm{m}}=\frac{\mathrm{d} \varepsilon}{\mathrm{dt}}=\frac{\mathrm{d} \varepsilon}{\mathrm{d} \lambda} \frac{\mathrm{d} \lambda}{\mathrm{dt}} \quad \text { ou } \\
& \dot{\varepsilon}_{\mathrm{m}}=\lambda^{2 \mathrm{~m}-1} \dot{\lambda}
\end{aligned}
$$

ou

$$
\dot{\varepsilon}_{\mathrm{m}}=\lambda^{2 \mathrm{~m}} \lambda^{-1} \frac{\dot{\ell}_{\mathrm{c}}}{\ell_{\mathrm{r}}}=\lambda^{2 \mathrm{~m}} \frac{\ell_{\mathrm{r}}}{\ell_{\mathrm{c}}} \frac{\dot{\ell}_{\mathrm{c}}}{\ell_{\mathrm{r}}}=\lambda^{2 \mathrm{~m}} \frac{\dot{\ell}_{\mathrm{c}}}{\ell_{\mathrm{c}}}
$$


chamando de taxa instantânea de deformação, que independe da configuração de referência, a relação:

$$
\dot{\mathrm{d}}=\frac{\dot{\ell}_{\mathrm{c}}}{\ell_{\mathrm{c}}}
$$

resulta uma família de taxas de deformação, dada por:

$$
\dot{\varepsilon}_{\mathrm{m}}=\lambda^{2 \mathrm{~m}} \dot{\mathrm{d}}
$$

Quando as deformações são pequenas, pode-se considerar que $\lambda \cong 1 \mathrm{e}$ todas as taxas de deformação se confundem.

No caso da deformação linear $(m=1 / 2)$, tem-se, com ajuda de 3.12:

$$
\dot{\varepsilon}=\dot{\lambda}
$$

A tensão de Cauchy e a tensão nominal ou de engenharia são definidas respectivamente por:

$$
\begin{aligned}
& \sigma_{c}=\frac{N}{A_{c}} \\
& \sigma_{N}=\frac{N}{A_{r}}
\end{aligned}
$$

Utilizando-se a potência da força $\mathrm{N}$ por unidade de volume de referência pode-se obter outras definições de tensão. $O$ trabalho total da força $\mathrm{N}$ que age na fibra é dado por: 


$$
\mathrm{W}_{\mathrm{T}}=\mathrm{N} \Delta \ell=\mathrm{N}\left(\ell_{\mathrm{r}}-\ell_{\mathrm{r}}\right)
$$

logo o trabalho por unidade de volume de referência é:

$$
\mathrm{W}=\frac{\mathrm{N}\left(\ell_{\mathrm{c}}-\ell_{\mathrm{r}}\right)}{\mathrm{V}_{\mathrm{r}}}
$$

Diferenciando o trabalho W no tempo resulta a potência da força $\mathrm{N}$ por unidade de volume de referência, dada por:

$$
\dot{\mathrm{W}}=\frac{\mathrm{N}}{\mathrm{A}_{\mathrm{r}} \ell_{\mathrm{r}}} \dot{\ell}_{\mathrm{o}}
$$

mas

$$
\begin{aligned}
& \mathrm{N}=\sigma_{\mathrm{c}} \mathrm{A}_{\mathrm{c}} \quad \text { e } \quad \dot{\ell}_{\mathrm{c}}=\ell_{\mathrm{c}} \dot{\mathrm{d}} \quad \text {,então } \\
& \dot{\mathrm{W}}=\frac{\sigma_{\mathrm{c}} \mathrm{A}_{\mathrm{c}}}{\mathrm{A}_{\mathrm{r}} \ell_{\mathrm{r}}} \ell_{\mathrm{c}} \dot{\mathrm{d}} \quad \text { ou } \\
& \dot{\mathrm{W}}=\frac{\sigma_{\mathrm{c}} \mathrm{V}_{\mathrm{c}}}{\mathrm{V}_{\mathrm{r}}} \dot{\mathrm{d}} \quad \text { ou } \\
& \dot{\mathrm{W}}=\sigma_{\mathrm{KT}} \dot{\mathrm{d}}
\end{aligned}
$$

e

$$
\sigma_{\mathrm{KT}}=\sigma_{\mathrm{c}} \frac{\mathrm{V}_{\mathrm{c}}}{\mathrm{V}_{\mathrm{r}}}
$$

onde $\sigma_{\mathrm{KT}}$ é chamada de tensão de Kirchhoff-Treffz. Esta tensão é a tensão de Cauchy corrigida devido à variação de volume. 
Pode-se definir uma família de tensões $\sigma_{\mathrm{m}}$ conjugada com a família de deformações, lembrando que para que haja dualidade entre a medida de tensão e a de deformação, a potência dos esforços externos dada pela eq. 3.24, deve ser igual a potência dos esforços internos dada por;

$$
\dot{\mathrm{W}}=\sigma_{\mathrm{m}} \dot{\varepsilon}_{\mathrm{m}}
$$

então:

$$
\sigma_{\mathrm{KT}} \dot{\mathrm{d}}=\sigma_{\mathrm{m}} \dot{\varepsilon}_{\mathrm{m}} \quad \text {, com auxílio da eq. } 3.18
$$

resulta:

$$
\sigma_{m}=\lambda^{-2 m} \sigma_{K T}
$$

com auxílio de $3.25,3.19,3.20$ e 3.9 vem:

$$
\sigma_{m}=\lambda^{-2 m+1} \sigma_{N}
$$

Desta família de tensões pode-se destacar alguns membros famosos:

a) Segunda tensão de Piola-Kirchhoff $(m=1)$

$$
\sigma_{1}=\lambda^{-1} \sigma_{\mathrm{N}}
$$

Esta tensão é conjugada com a deformação de Green.

b) Tensão nominal ou de engenharia $(m=1 / 2)$ 


$$
\sigma_{1 / 2}=\sigma_{\mathrm{N}}
$$

que é conjugada com a deformação linear ou de engenharia. Esta tensão é também conhecida como tensão de Biot.

c) Tensão de Kirchhoff-Treffz $(m=0)$

$$
\sigma_{0}=\lambda \sigma_{\mathrm{N}}=\sigma_{\mathrm{KT}}
$$

que é conjugada com a deformação de Hencky.

Analogamente pode-se obter uma família de taxas de tensionamento, pela derivação da eq. 3.28 no tempo, resultando:

$$
\dot{\sigma}_{m}=\lambda^{1-2 m} \dot{\sigma}_{N}+\sigma_{N}(1-2 m) \lambda^{-2 m} \dot{\lambda}
$$

Para $m=1 / 2$, isto é, tensão nominal ou de engenharia tem-se:

$$
\dot{\sigma}_{1 / 2}=\dot{\sigma}_{\mathrm{N}}
$$

Para $m=1$, isto é, $2^{\underline{a}}$ tensão de Piola-Kirchhoff tem-se:

$$
\dot{\sigma}_{1}=\lambda^{-1} \dot{\sigma}_{\mathrm{N}}-2 \sigma_{\mathrm{N}} \dot{\lambda}
$$

Cabe ressaltar que no caso de pequenas deformações $(\lambda \cong 1)$, todos os membros da família de tensões se confundem, mas as taxas de tensionamento diferem entre si.

Define-se o módulo de rigidez tangente do material como: 


$$
D=\frac{d \sigma}{d \varepsilon}
$$

então

$$
\dot{\sigma}_{\mathrm{m}}=\mathrm{D}_{\mathrm{m}} \dot{\varepsilon}_{\mathrm{m}}
$$

aplicando a expressão para $m=1 / 2$, ou seja, tensão de engenharia, vem:

$$
\dot{\sigma}_{\mathrm{N}}=\mathrm{D}_{\mathrm{N}} \dot{\varepsilon}_{1 / 2}
$$

e com auxílio de (3.18a), tem-se:

$$
\dot{\sigma}_{\mathrm{N}}=\mathrm{D}_{\mathrm{N}} \dot{\lambda}
$$

então, de (3.36), (3.17), (3.32) e (3.38) resulta a expressão de uma família de módulos de rigidez, dependentes da definição de tensão e deformação, dada por:

$$
D_{m}=\lambda^{-4 m+2} D_{N}+\sigma_{N}(1-2 m) \lambda^{-4 m+1}
$$

No caso da tensão nominal e deformação linear $(m=1 / 2)$, tem-se:

$$
\mathrm{D}_{1 / 2}=\mathrm{D}_{\mathrm{N}}
$$

No caso da tensão de Piola-Kirchhoff e deformação de Green $(m=1)$, tem-se:

$$
D_{1}=\lambda^{-2} D_{N}-\lambda^{-3} \sigma_{N}
$$


Numa formulação consistente deve-se usar medidas de tensões e deformações conjugadas, tais como:

- deformação de Green e $2^{\mathrm{a}}$ tensão de Piola-Kirchhoff.

- deformação linear e tensão nominal ou de engenharia.

BATHE (1996) recomenda o uso de tensões e deformações conjugadas e objetivas. Diz-se que as tensões e deformações são objetivas quando são invariantes sob movimento de corpo rígido. BATHE mostra que a deformação de Green e a segunda tensão de Piola-Kirchhoff são medidas objetivas, enquanto que a tensão nominal e a deformação linear são medidas não objetivas quando rotações finitas estão envolvidas.

A deformação de Green e a segunda tensão de Piola-Kirchhoff são as medidas mais usadas na análise estrutural não-linear, porque elas são conjugadas, objetivas e medidas de deformação e tensão finitas completas, conforme ressalta ORAN \& KASSIMALI (1976). Entretanto, elas não são medidas geométricas, não possuindo significado físico aparente, sendo então, difícil compatibilizá-las com as condições naturais da estrutura. Para resolver este problema, deve-se expressar a segunda tensão de Piola-Kirchhoff em função da tensão de Cauchy, que é mensurável, levando-se a trabalhosas transformações.

Já a tensão nominal e a deformação linear são medidas geométricas, têm direções definidas, podem ser facilmente compatibilizadas com as condições de contorno naturais e podem fazer uso direto das constantes dos materiais obtidas de experimentos com pequenas deformações. Entretanto, a tensão nominal e a deformação linear só são objetivas para rotações infinitesimais. Todavia, para problemas geometricamente não-lineares, a estrutura está, de fato, submetida a deformações infinitesimais, medidas em relação a um sistema de coordenadas solidário ao elemento, enquanto a 
estrutura sofre grandes translações e rotações as quais não causam quaisquer deformações objetivas.

Como será demonstrado posteriormente, quando se utiliza um sistema de coordenadas-deslocamentos corrotacional, a tensão nominal e deformação linear tornam-se objetivas.

Assim, neste trabalho adotar-se-á o sistema de coordenadasdeslocamento corrotacional solidário ao elemento, e usará a deformação linear e a tensão nominal ou de engenharia. Aplicando-se as relações deformaçãodeslocamento no campo de deslocamento local, definido em função apenas dos graus de liberdade naturais, obtém-se as deformações de engenharia objetivas. Os deslocamentos de corpo rígido são levados em conta através de uma transformação entre o sistema de coordenadas Lagrangiano ou cartesiano, que descreve a configuração indeformada, e o sistema de coordenadas corrotacional, que descreve a configuração deformada.

Como serão utilizados como medidas de deformações e tensões a deformação linear e a tensão de engenharia, respectivamente, a partir de agora elas serão referidas simplesmente como $\varepsilon$ e $\sigma$, isto é:

$$
\varepsilon=\varepsilon_{1 / 2}=\lambda-1
$$

e

$$
\sigma=\sigma_{1 / 2}=\sigma_{\mathrm{N}}
$$

cujas derivadas no tempo são:

$$
\dot{\varepsilon}=\dot{\varepsilon}_{1 / 2}=\dot{\lambda}
$$

e

$$
\dot{\sigma}=\dot{\sigma}_{1 / 2}=\dot{\sigma}_{N}
$$




\subsection{Definição do Modelo Elemental. Graus de Liberdade e Compatibilidade Geométrica}

Seja uma estrutura em pórtico plano formado por barras prismáticas retas em sua configuração inicial ou de referência, relacionada a um referencial global cartesiano $\mathrm{x}, \mathrm{y}$. Os nós do pórtico possuem três graus de liberdade cartesianos, os deslocamentos $\mathrm{u}$ e $\mathrm{v}$ segundo os eixos cartesianos $\mathrm{x} e$ $y$, respectivamente, e a rotação $\theta$ medida no sentido anti-horário.

Considere-se um elemento qualquer deste pórtico na configuração de referência ${ }^{\circ} \mathrm{A}^{\circ} \mathrm{B}$. Seja $\ell_{\mathrm{r}}$ o comprimento deste elemento nesta configuração e $\varphi_{\mathrm{r}}$ o ângulo que este elemento faz com o eixo $\mathrm{x}$, conforme mostrado na Fig. 3.4. Introduz-se nesta configuração de referência um sistema de coordenadas $x_{r}, y_{r}$ centrado no elemento.

Para um determinado nível de carregamento esta barra encontra-se numa nova configuração ${ }^{1} \mathrm{~A}^{\mathrm{l}} \mathrm{B}$, chamada configuração deformada ou atual. Nesta nova configuração introduz-se um sistema local de coordenadas $x_{c}, y_{c}$ solidário com o elemento, chamado sistema de coordenadas corrotacional, centrado na corda que une as extremidades do elemento. $O$ comprimento desta corda é $\ell_{\mathrm{c}}$ e faz um ângulo $\varphi_{\mathrm{c}}$ com o eixo cartesiano $\mathrm{x}$.

As coordenadas-deslocamento no sistema cartesiano global são:

$$
\mathbf{p}_{i}=\left\{\begin{array}{l}
p_{1} \\
p_{2} \\
p_{3} \\
p_{4} \\
p_{5} \\
p_{6}
\end{array}\right\}
$$




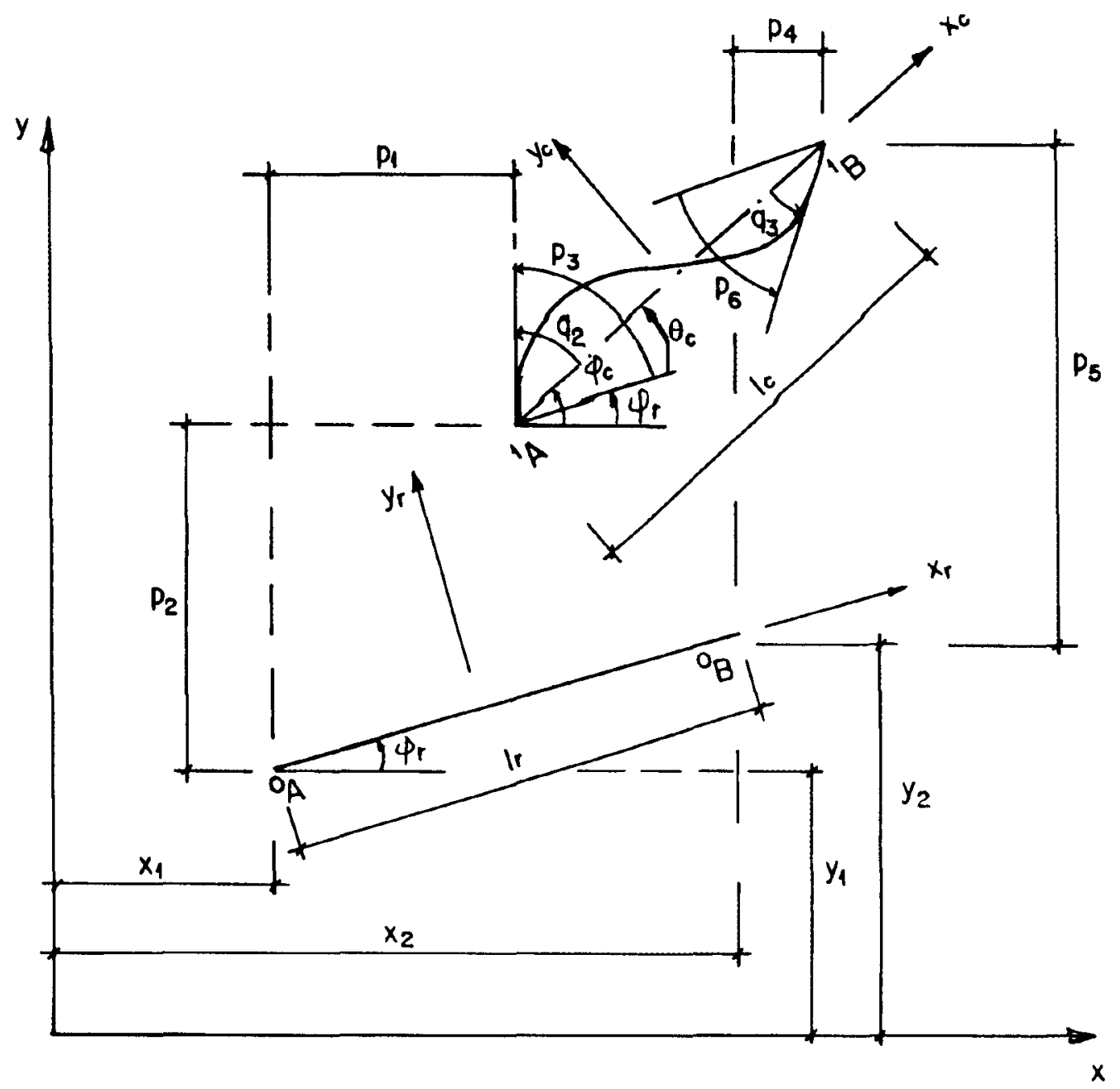

FIGURA 3.4 - Modelo Elemental. Sistemas de Coordenadas.

Graus de Liberdade

As coordenadas-deslocamento naturais no sistema local corrotacional são:

$$
\mathbf{q}_{\alpha}=\left\{\begin{array}{l}
\mathrm{q}_{1} \\
\mathrm{q}_{2} \\
\mathrm{q}_{3}
\end{array}\right\}
$$

onde $\mathrm{q}_{1}$ mede a variação do comprimento da corda $\left(\ell_{\mathrm{c}}-\ell_{\mathrm{r}}\right), \mathrm{q}_{2}$ e $\mathrm{q}_{3}$ medem as rotações das extremidades da barra, independentes da rotação de corpo rígido $\theta_{c}$, dada por: 


$$
\theta_{\mathrm{c}}=\varphi_{\mathrm{c}}-\varphi_{\mathrm{r}}
$$

Com o objetivo de possibilitar a mudança do sistema de coordenadas local para o sistema global deve-se relacionar os graus de liberdades naturais $q_{\alpha}, \alpha=1,3$, com os graus de liberdade em coordenadas globais cartesianas $\mathrm{p}_{\mathrm{i}}, \mathrm{i}$ $=1,6$.

Sejam $\left(\mathrm{x}_{1}, \mathrm{y}_{1}\right)$ e $\left(\mathrm{x}_{2}, \mathrm{y}_{2}\right)$ as coordenadas dos nós ${ }^{\circ} \mathrm{A}$ e ${ }^{\circ} \mathrm{B}$, respectivamente, no sistema global x,y. então, da Fig. 3.4 tem-se:

$$
\begin{aligned}
& \ell_{\mathrm{r}}=\left[\left(\mathrm{x}_{2}-\mathrm{x}_{1}\right)^{2}+\left(\mathrm{y}_{2}-\mathrm{y}_{1}\right)^{2}\right]^{1 / 2} \\
& \ell_{\mathrm{c}}=\left[\left(\mathrm{x}_{2}-\mathrm{x}_{1}+\mathrm{p}_{4}-\mathrm{p}_{1}\right)^{2}+\left(\mathrm{y}_{2}-\mathrm{y}_{1}+\mathrm{p}_{5}-\mathrm{p}_{2}\right)^{2}\right]^{1 / 2} \\
& \cos \varphi_{\mathrm{c}}=\frac{\mathrm{x}_{2}+\mathrm{p}_{4}-\mathrm{x}_{1}-\mathrm{p}_{1}}{\ell_{\mathrm{c}}} \\
& \operatorname{sen} \varphi_{\mathrm{c}}=\frac{\mathrm{y}_{2}+\mathrm{p}_{5}-\mathrm{y}_{1}-\mathrm{p}_{2}}{\ell_{\mathrm{c}}}
\end{aligned}
$$

Da eq. 3.48 , tem-se:

$$
\begin{aligned}
& \operatorname{sen} \theta_{c}=\operatorname{sen}\left(\varphi_{c}-\varphi_{r}\right) \quad \text { ou } \\
& \operatorname{sen} \theta_{c}=\operatorname{sen} \varphi_{c} \cos \varphi_{r}-\operatorname{sen} \varphi_{r} \cos \varphi_{c}
\end{aligned}
$$

mas, 


$$
\cos \varphi_{r}=\frac{x_{2}-x_{1}}{\ell_{r}}
$$

e

$$
\operatorname{sen} \varphi_{r}=\frac{y_{2}-y_{1}}{\ell_{r}}
$$

então, com auxílio de $3.51,3.52,3.55$ e 3.56 resulta:

$$
\begin{aligned}
\operatorname{sen} \theta_{c}= & \frac{y_{2}+p_{5}-y_{1}-p_{2}}{\ell_{c}} \frac{x_{2}-x_{1}}{\ell_{r}} \\
& -\frac{x_{2}+p_{4}-x_{1}-p_{1}}{\ell_{c}} \frac{y_{2}-y_{1}}{\ell_{r}}
\end{aligned}
$$

ou ainda,

$$
\begin{aligned}
\theta_{c}=\operatorname{arcsen} & {\left[\frac{\left(y_{2}+p_{5}-y_{1}-p_{2}\right)\left(x_{2}-x_{1}\right)}{\ell_{c} \ell_{r}}\right.} \\
& \left.-\frac{\left(x_{2}+p_{4}-x_{1}-p_{1}\right)\left(y_{2}-y_{1}\right)}{\ell_{c} \ell_{r}}\right]
\end{aligned}
$$

e

$$
\varphi_{c}=\operatorname{arctg}\left[\frac{\left(y_{2}-y_{1}+p_{5}-p_{2}\right)}{\left(x_{2}-x_{1}+p_{4}-p_{1}\right)}\right]
$$

Define-se como matriz instantânea de mudança de coordenadas a matriz $\mathbf{B}$ que relaciona as taxas de deslocamento nas coordenadas naturais $\left(d q_{\alpha}\right)$ com as taxas de deslocamentos nas coordenadas cartesianas $\left(d p_{i}\right)$, isto é: 


$$
\mathrm{dq}_{\alpha}=\mathbf{B} \mathrm{dp}_{\mathrm{i}}
$$

Os elementos da matriz B são obtidos da derivação $q_{\alpha, i}$, ou seja:

$$
\mathrm{q}_{\alpha, \mathrm{i}}=\frac{\mathrm{dq}_{\alpha}}{\mathrm{dp}_{\mathrm{i}}}=\mathbf{B}
$$

Assim:

$$
\begin{aligned}
q_{1,1}= & \frac{d q_{1}}{d p_{1}}=\frac{d}{d p_{1}}\left\{\left[\left(x_{2}-x_{1}+p_{4}-p_{1}\right)^{2}+\right.\right. \\
& \left.\left.\left(y_{2}-y_{1}+p_{5}-p_{2}\right)^{2}\right]^{1 / 2}-\ell_{r}\right\} \\
q_{1,1}= & \frac{1}{2}\left[\left(x_{2}-x_{1}+p_{4}-p_{1}\right)^{2}+\left(y_{2}-y_{1}+p_{5}-p_{2}\right)^{2}\right]^{-1 / 2} \\
& \cdot\left[2\left(x_{2}-x_{1}+p_{4}-p_{1}\right)(-1)\right] \\
q_{1,1}= & \frac{1}{\ell}\left(x_{2}-x_{1}+p_{4}-p_{1}\right)(-1) \\
q_{1,1}= & -\cos \varphi_{c} \\
q_{1,2}= & \frac{d q_{1}}{d p_{2}} \\
q_{1,2}= & \frac{1}{2}\left[\left(x_{2}-x_{1}+p_{4}-p_{1}\right)^{2}+\left(y_{2}-y_{1}+p_{5}-p_{2}\right)^{2}\right]^{-1 / 2} \\
& \cdot\left[2\left(y_{2}-y_{1}+p_{5}-p_{2}\right)(-1)\right]
\end{aligned}
$$




$$
\begin{aligned}
& \mathrm{q}_{1,2}=\frac{1}{\ell_{\mathrm{c}}}\left(\mathrm{y}_{2}-\mathrm{y}_{1}+\mathrm{p}_{\mathrm{s}}-\mathrm{p}_{2}\right)(-1) \\
& \mathrm{q}_{1,2}=-\operatorname{sen} \varphi_{\mathrm{c}}
\end{aligned}
$$

Das equações 3.47, 3.49, 3.50 tẹm-se;

analogamente tem-se:

$$
\mathrm{q}_{1,3}=0
$$

analogamente tem-se:

$$
\begin{aligned}
& \mathrm{q}_{1,4}=\cos \varphi_{c} \\
& \mathrm{q}_{1,5}=\operatorname{sen} \varphi_{c} \\
& \mathrm{q}_{1,6}=0
\end{aligned}
$$

Da figura 3.4 tem-se:

$$
\mathrm{q}_{2}=\mathrm{p}_{3}-\theta_{\mathrm{c}}
$$

então, com auxílio de 3.48 e $3.58 \mathrm{a}$, vem:

$$
\mathbf{q}_{2,1}=\frac{\mathrm{d}}{\mathrm{dp}_{1}}\left\{\mathrm{p}_{3}+\varphi_{\mathrm{r}}-\operatorname{arctg}\left[\frac{\mathrm{y}_{2}-\mathrm{y}_{1}+\mathrm{p}_{5}-\mathrm{p}_{2}}{\mathrm{x}_{2}-\mathrm{x}_{1}+\mathrm{p}_{4}-\mathrm{p}_{1}}\right]\right\}
$$


resultando:

$$
\mathrm{q}_{2,1}=-\frac{\operatorname{sen} \varphi_{\mathrm{c}}}{\ell_{\mathrm{c}}}
$$

analogamente vem:

$$
\begin{aligned}
& \mathrm{q}_{2,2}=\frac{\cos \varphi_{c}}{\ell_{c}} \\
& \mathrm{q}_{2,3}=1 \\
& \mathrm{q}_{2,4}=\frac{\operatorname{sen} \varphi_{c}}{\ell_{c}} \\
& q_{2,5}=-\frac{\cos \varphi_{c}}{\ell_{c}} \\
& q_{2,6}=0
\end{aligned}
$$

Da figura 3.4 tem-se:

$$
\mathrm{q}_{3}=\mathrm{p}_{6}-\theta_{\mathrm{c}}
$$

então, com auxílio de 3.48 e $3.58 \mathrm{a}$, vem:

$$
q_{3,1}=\frac{d}{d p_{1}}\left\{p_{6}+\varphi_{r}-\operatorname{arctg}\left[\frac{y_{2}-y_{1}+p_{5}-p_{2}}{x_{2}-x_{1}+p_{4}-p_{1}}\right]\right\}
$$


resultando:

$$
\mathrm{q}_{3,1}=-\frac{\operatorname{sen} \varphi_{\mathrm{c}}}{\ell_{\mathrm{c}}}
$$

analogamente vem:

$$
\begin{aligned}
& \mathrm{q}_{3,2}=\frac{\cos \varphi_{\mathrm{c}}}{\ell_{\mathrm{c}}} \\
& \mathrm{q}_{3,3}=0 \\
& \mathrm{q}_{3,4}=\frac{\operatorname{sen} \varphi_{\mathrm{c}}}{\ell_{\mathrm{c}}} \\
& \mathrm{q}_{3,5}=-\frac{\cos \varphi_{\mathrm{c}}}{\ell_{\mathrm{c}}} \\
& \mathrm{q}_{3,6}=1
\end{aligned}
$$

Assim a equação (3.59) pode ser explicitada como:

$$
\left\{\begin{array}{l}
\mathrm{dq}_{1} \\
\mathrm{dq}_{2} \\
\mathrm{dq}_{3}
\end{array}\right\}=\left[\begin{array}{cccccc}
-\cos \varphi_{\mathrm{c}} & -\operatorname{sen} \varphi_{\mathrm{c}} & 0 & \cos \varphi_{\mathrm{c}} & \operatorname{sen} \varphi_{\mathrm{c}} & 0 \\
-\frac{\operatorname{sen} \varphi_{\mathrm{c}}}{\ell_{\mathrm{c}}} & \frac{\cos \varphi_{\mathrm{c}}}{\ell_{\mathrm{c}}} & 1 & \frac{\operatorname{sen} \varphi_{\mathrm{c}}}{\ell_{\mathrm{c}}} & -\frac{\cos \varphi_{\mathrm{c}}}{\ell_{\mathrm{c}}} & 0 \\
-\frac{\operatorname{sen} \varphi_{\mathrm{c}}}{\ell_{\mathrm{c}}} & \frac{\cos \varphi_{\mathrm{c}}}{\ell_{\mathrm{c}}} & 0 & \frac{\operatorname{sen} \varphi_{\mathrm{c}}}{\ell_{\mathrm{c}}} & -\frac{\cos \varphi_{\mathrm{c}}}{\ell_{\mathrm{c}}} & 1
\end{array}\right]\left[\begin{array}{l}
\mathrm{dp_{1 }} \\
\mathrm{dp}_{2} \\
\mathrm{dp}_{3} \\
\mathrm{dp}_{4} \\
\mathrm{dp}_{5} \\
\mathrm{dp}_{6}
\end{array}\right\}
$$


Cabe observar que a matriz B de dimensão $3 \times 6$ é uma matriz instantânea de mudança de coordenadas para uma particular configuração da estrutura. Naturalmente esta matriz não é linear, uma vez que o ângulo $\varphi_{c}$ e $o$ comprimento da corda $\ell_{\mathrm{c}}$ dependem dos deslocamentos nodais da estrutura.

Esta matriz B pode ser escrita como um produto dado por:

$$
\mathbf{B}=\overline{\mathbf{B}} \mathbf{T}
$$

ou seja:

$\mathbf{B}=\left[\begin{array}{cccccc}-1 & 0 & 0 & 1 & 0 & 0 \\ 0 & 1 / \ell_{\mathrm{c}} & 1 & 0 & -1 / \ell_{\mathrm{c}} & 0 \\ 0 & 1 / \ell_{\mathrm{c}} & 0 & 0 & -1 / \ell_{\mathrm{c}} & 1\end{array}\right] \cdot\left[\begin{array}{cccccc}\cos \varphi_{\mathrm{c}} & \operatorname{sen} \varphi_{\mathrm{c}} & 0 & 0 & 0 & 0 \\ -\operatorname{sen} \varphi_{\mathrm{c}} & \cos \varphi_{\mathrm{c}} & 0 & 0 & 0 & 0 \\ 0 & 0 & 1 & 0 & 0 & 0 \\ 0 & 0 & 0 & \cos \varphi_{\mathrm{c}} & \operatorname{sen} \varphi_{\mathrm{c}} & 0 \\ 0 & 0 & 0 & -\operatorname{sen} \varphi_{\mathrm{c}} & \cos \varphi_{\mathrm{c}} & 0 \\ 0 & 0 & 0 & 0 & 0 & 1\end{array}\right]$

então:

$$
\overline{\mathbf{B}}=\left[\begin{array}{cccccc}
-1 & 0 & 0 & 1 & 0 & 0 \\
0 & 1 / \ell_{c} & 1 & 0 & -1 / \ell_{c} & 0 \\
0 & 1 / \ell_{c} & 0 & 0 & -1 / \ell_{c} & 1
\end{array}\right]
$$

e

$$
\mathbf{T}=\left[\begin{array}{cccccc}
\cos \varphi_{\mathrm{c}} & \operatorname{sen} \varphi_{\mathrm{c}} & 0 & 0 & 0 & 0 \\
-\operatorname{sen} \varphi_{\mathrm{c}} & \cos \varphi_{\mathrm{c}} & 0 & 0 & 0 & 0 \\
0 & 0 & 1 & 0 & 0 & 0 \\
0 & 0 & 0 & \cos \varphi_{\mathrm{c}} & \operatorname{sen} \varphi_{\mathrm{c}} & 0 \\
0 & 0 & 0 & -\operatorname{sen} \varphi_{\mathrm{c}} & \cos \varphi_{\mathrm{c}} & 0 \\
0 & 0 & 0 & 0 & 0 & 1
\end{array}\right]
$$


A matriz $\mathbf{T}$ de dimensão $6 \times 6$ é a tradicional matriz de mudança de coordenadas cartesianas, isto é, relaciona deslocamento cartesianos globais com deslocamentos cartesianos locais na configuração deformada.

A matriz $\overline{\mathbf{B}}$ de dimensão $3 \times 6$ é a forma local de $\mathbf{B}$, isto é, relaciona os deslocamentos locais cartesianos da barra na configuração deformada com os deslocamentos locais naturais.

Para obtenção da matriz de rigidez tangente do elemento será necessária a segunda derivada parcial da energia de deformação em relação aos graus de liberdade. Será portanto, necessário conhecer as relações diferenciais de $2^{\mathrm{a}}$ ordem entre os graus de liberdade naturais da barra $\left(\mathrm{q}_{\alpha}\right)$ e graus de liberdade globais $\left(\mathrm{p}_{\mathrm{i}}\right)$, visto que, precisa-se conhecer a matriz de rigidez tangente da estrutura nas coordenadas globais. A seguir serão determinadas estas derivadas indicadas por:

$$
\mathbf{q}_{\alpha, \mathrm{j}}=\frac{\partial \mathbf{q}_{\alpha}}{\partial \mathrm{p}_{\mathrm{i}} \partial \mathrm{p}_{\mathrm{j}}}
$$

$\operatorname{com} \alpha=1,3, \quad i=1,6, \quad j=1,6$

Assim:

$$
\mathbf{q}_{1,11}=\frac{\partial^{2} \mathbf{q}_{1}}{\partial p_{1} \partial p_{1}}=\frac{\partial}{\partial p_{1}}\left(\frac{\partial \mathbf{q}_{1}}{\partial p_{1}}\right)
$$

com auxilio de (3.60a), vem:

$$
\mathbf{q}_{1,11}=\frac{\partial}{\partial \mathrm{p}_{1}}\left(-\cos \varphi_{\mathrm{c}}\right)=\operatorname{sen} \varphi_{\mathrm{c}} \cdot \frac{\partial \varphi_{\mathrm{c}}}{\partial \mathrm{p}_{1}}
$$

a partir (3.58a) tem-se: 


$$
\begin{aligned}
\frac{\partial p_{c}}{\partial p_{1}} & =\frac{\partial}{\partial p_{1}}\left\{\operatorname{arctg}\left[\frac{y_{2}-y_{1}+p_{5}-p_{2}}{x_{2}-x_{1}+p_{4}-p_{1}}\right]\right\} \\
& =\frac{1}{1+\left(\frac{y_{2}-y_{1}+p_{5}-p_{2}}{x_{2}-x_{1}+p_{4}-p_{1}}\right)^{2}} \cdot \frac{-\left(y_{2}-y_{1}+p_{5}-p_{2}\right)}{\left(x_{2}-x_{1}+p_{4}-p_{1}\right)^{2}} \cdot(-1) \\
& =\frac{\left(x_{2}-x_{1}+p_{4}-p_{1}\right)^{2}}{\left(x_{2}-x_{1}+p_{4}-p_{1}\right)^{2}+\left(y_{2}-y_{1}+p_{5}-p_{2}\right)^{2}} \cdot \frac{\left(y_{2}-y_{1}+p_{5}-p_{2}\right)}{\left(x_{2}-x_{1}+p_{4}-p_{1}\right)^{2}} \\
& =\frac{\left(y_{2}-y_{1}+p_{5}-p_{2}\right)}{\left(x_{2}-x_{1}+p_{4}-p_{1}\right)^{2}+\left(y_{2}-y_{1}+p_{5}-p_{2}\right)^{2}}
\end{aligned}
$$

com auxílio de 3.50 vem:

$$
\frac{\partial \varphi_{\mathrm{c}}}{\partial \mathrm{p}_{1}}=\frac{\mathrm{y}_{2}-\mathrm{y}_{1}+\mathrm{p}_{\mathrm{s}}-\mathrm{p}_{2}}{\ell_{\mathrm{c}}^{2}}=\frac{1}{\ell_{\mathrm{c}}} \cdot \frac{\mathrm{y}_{2}-\mathrm{y}_{1}+\mathrm{p}_{5}-\mathrm{p}_{2}}{\ell_{\mathrm{c}}}
$$

com auxílio de 3.51 , resulta:

$$
\frac{\partial \varphi_{\mathrm{c}}}{\partial \mathrm{p}_{1}}=\frac{\operatorname{sen} \varphi_{\mathrm{c}}}{\ell_{\mathrm{c}}}
$$

substituindo (3.68) em (3.67) vem:

$$
\mathrm{q}_{1,11}=\frac{\operatorname{sen}^{2} \varphi_{\mathrm{c}}}{\ell_{\mathrm{c}}}
$$




$$
\mathrm{q}_{1,12}=\frac{\partial^{2} \mathrm{q}_{1}}{\partial \mathrm{p}_{1} \partial \mathrm{p}_{2}}=\frac{\partial}{\partial \mathrm{p}_{2}}\left(\frac{\partial \mathrm{q}_{1}}{\partial \mathrm{p}_{1}}\right)
$$

com auxílio de (3.60a), vem:

$$
\mathrm{q}_{1,12}=\frac{\partial}{\partial \mathrm{p}_{2}}\left(-\cos \varphi_{\mathrm{c}}\right)=\operatorname{sen} \varphi_{\mathrm{c}} \cdot \frac{\partial \varphi_{\mathrm{c}}}{\partial \mathrm{p}_{2}}
$$

a partir de (3.58a), de forma análoga a obtenção de (3.68), tem-se:

$$
\frac{\partial \varphi_{c}}{\partial p_{2}}=-\frac{\cos \varphi_{c}}{\ell_{c}}
$$

substituindo (3.71) em (3.70) vem:

$$
\mathrm{q}_{1,12}=-\frac{\operatorname{sen} \varphi_{\mathrm{c}} \cos \varphi_{\mathrm{c}}}{\ell_{\mathrm{c}}}
$$

Analogamente, obtém-se:

$$
\begin{aligned}
& \mathrm{q}_{1,13}=0 \\
& \mathrm{q}_{1,14}=-\frac{\operatorname{sen}^{2} \varphi_{\mathrm{c}}}{\ell_{\mathrm{c}}} \\
& \mathrm{q}_{1,15}=\frac{\operatorname{sen} \varphi_{\mathrm{c}} \cos \varphi_{\mathrm{c}}}{\ell_{\mathrm{c}}} \\
& \mathrm{q}_{1,16}=0
\end{aligned}
$$




$$
\mathrm{q}_{1,21}=\frac{\partial^{2} \mathrm{q}_{1}}{\partial \mathrm{p}_{2} \partial \mathrm{p}_{1}}=\frac{\partial}{\partial \mathrm{p}_{1}}\left(\frac{\partial \mathrm{q}_{1}}{\partial \mathrm{p}_{2}}\right)
$$

com auxílio de (3.60b), vem:

$$
q_{1,12}=\frac{\partial}{\partial p_{1}}\left(-\operatorname{sen} \varphi_{c}\right)=-\cos \varphi_{c} \frac{\partial \varphi_{c}}{\partial p_{1}}
$$

substituindo (3.68) em (3.77) vem:

$$
\begin{aligned}
& \mathbf{q}_{1,21}=-\frac{\operatorname{sen} \varphi_{c} \cos \varphi_{c}}{\ell_{c}} \\
& q_{1,22}=\frac{\partial^{2} q_{1}}{\partial p_{2} \partial p_{2}}=\frac{\partial}{\partial p_{2}}\left(\frac{\partial q_{1}}{\partial p_{2}}\right) \quad \text { ou } \\
& q_{1,22}=\frac{\partial}{\partial p_{2}}\left(-\operatorname{sen} \varphi_{c}\right)=-\cos \varphi_{c} \frac{\partial \varphi_{c}}{\partial p_{2}}
\end{aligned}
$$

substituindo (3.71) em (3.79) vem:

$$
\mathrm{q}_{1,22}=\frac{\cos ^{2} \varphi_{\mathrm{c}}}{\ell_{\mathrm{c}}}
$$

Analogamente, obtém-se:

$$
\begin{aligned}
& \mathrm{q}_{1,23}=0 \\
& \mathrm{q}_{1,24}=\frac{\operatorname{sen} \varphi_{\mathrm{c}} \cos \varphi_{\mathrm{c}}}{\ell_{\mathrm{c}}}
\end{aligned}
$$




$$
\mathrm{q}_{1,25}=-\frac{\cos ^{2} \varphi_{\mathrm{c}}}{\ell_{\mathrm{c}}}
$$

$$
\mathrm{q}_{1,26}=0
$$

$$
\mathrm{q}_{1,31}=\frac{\partial^{2} \mathrm{q}_{1}}{\partial \mathrm{p}_{3} \partial \mathrm{p}_{1}}=\frac{\partial}{\partial \mathrm{p}_{1}}\left(\frac{\partial \mathrm{q}_{1}}{\partial \mathrm{p}_{3}}\right)
$$

com auxílio de (3.60c), conclui-se que:

$$
\mathrm{q}_{1,31}=0
$$

Analogamente:

$$
\begin{aligned}
& \mathbf{q}_{1,32}=0 \\
& \mathbf{q}_{1,33}=0 \\
& \mathbf{q}_{1,34}=0 \\
& \mathbf{q}_{1,35}=0 \\
& \mathbf{q}_{1,36}=0 \\
& \mathbf{q}_{1,41}=\frac{\partial^{2} \mathbf{q}_{1}}{\partial \mathrm{p}_{4} \partial \mathrm{p}_{1}}=\frac{\partial}{\partial \mathrm{p}_{1}}\left(\frac{\partial \mathrm{q}_{1}}{\partial \mathrm{p}_{4}}\right)
\end{aligned}
$$

com auxílio de (3.60d), vem: 
90

$$
q_{1,41}=\frac{\partial}{\partial p_{1}}\left(\cos \varphi_{c}\right)=\operatorname{sen} \varphi_{c} \frac{\partial \varphi_{c}}{\partial p_{1}}
$$

substituindo (3.68) em (3.91), resulta:

$$
q_{1,41}=-\frac{\operatorname{sen}^{2} \varphi_{c}}{\ell_{c}}
$$

Analogamente, obtém-se:

$$
\mathbf{q}_{1,42}=\frac{\operatorname{sen} \varphi_{c} \cos \varphi_{c}}{\ell_{c}}
$$

$\mathbf{q}_{1,43}=0$

$$
\mathrm{q}_{\mathrm{i}, 44}=\frac{\operatorname{sen}^{2} \varphi_{\mathrm{c}}}{\ell_{\mathrm{c}}}
$$

$$
\mathbf{q}_{1,45}=-\frac{\operatorname{sen} \varphi_{c} \cos \varphi_{c}}{\ell_{c}}
$$

$$
\mathrm{q}_{1,46}=0
$$

$$
\mathrm{q}_{1,51}=\frac{\partial^{2} \mathrm{q}_{1}}{\partial \mathrm{p}_{5} \partial \mathrm{p}_{1}}=\frac{\partial}{\partial \mathrm{p}_{1}}\left(\frac{\partial \mathrm{q}_{1}}{\partial \mathrm{p}_{5}}\right)
$$

com auxílio de (3.60e), vem: 


$$
q_{1,51}=\frac{\partial}{\partial p_{1}}\left(\operatorname{sen} \varphi_{c}\right)=\cos \varphi_{c} \frac{\partial \varphi_{c}}{\partial p_{1}}
$$

substituindo (3.68) em (3.98) vem:

$$
\mathrm{q}_{1,51}=\frac{\operatorname{sen} \varphi_{\mathrm{c}} \cos \varphi_{\mathrm{c}}}{\ell_{\mathrm{c}}}
$$

Analogamente, obtém-se:

$$
\mathrm{q}_{1,52}=-\frac{\cos ^{2} \varphi_{\mathbf{c}}}{\ell_{\mathrm{c}}}
$$

$$
\mathrm{q}_{1,53}=0
$$

$$
\mathrm{q}_{1,54}=-\frac{\operatorname{sen} \varphi_{\mathrm{c}} \cos \varphi_{\mathrm{c}}}{\ell_{\mathrm{c}}}
$$

$$
\mathrm{q}_{1,55}=\frac{\cos ^{2} \varphi_{\mathrm{c}}}{\ell_{\mathrm{c}}}
$$

$$
\mathrm{q}_{1,56}=0
$$

$$
q_{1,61}=\frac{\partial^{2} q_{1}}{\partial p_{6} \partial p_{1}}=\frac{\partial}{\partial p_{1}}\left(\frac{\partial q_{1}}{\partial p_{6}}\right)
$$

com auxílio de (3.60f), conclui-se que:

$$
\mathrm{q}_{1,61}=0
$$


Analogamente:

$$
\begin{aligned}
& \mathrm{q}_{1,62}=0 \\
& \mathrm{q}_{1,63}=0 \\
& \mathrm{q}_{1,64}=0 \\
& \mathrm{q}_{1,65}=0 \\
& \mathrm{q}_{1,66}=0
\end{aligned}
$$

Estas derivadas podem ser agrupadas numa matriz 6x6 que é simétrica e será denominada $\mathbf{G}_{1}$, ou seja:

$$
\mathbf{G}_{1}=\frac{\partial^{2} q_{1}}{\partial p_{i} \partial p_{j}}
$$

ou de forma explícita:

$$
\mathbf{G}_{1}=\ell_{\mathrm{c}}^{-1}\left[\begin{array}{cccccc}
\operatorname{sen}^{2} \varphi_{\mathrm{c}} & -\operatorname{sen} \varphi_{\mathrm{c}} \cos \varphi_{\mathrm{c}} & 0 & -\operatorname{sen}^{2} \varphi_{\mathrm{c}} & \operatorname{sen} \varphi_{\mathrm{c}} \cos \varphi_{\mathrm{c}} & 0 \\
& \cos ^{2} \varphi_{\mathrm{c}} & 0 & \operatorname{sen} \varphi_{\mathrm{c}} \cos \varphi_{\mathrm{c}} & -\cos ^{2} \varphi_{\mathrm{c}} & 0 \\
& & 0 & 0 & 0 & 0 \\
& & & \operatorname{sen}^{2} \varphi_{\mathrm{c}} & -\operatorname{sen} \varphi_{\mathrm{c}} \cos \varphi_{\mathrm{c}} & 0 \\
& & & & \cos ^{2} \varphi_{\mathrm{c}} & 0 \\
& & & & & 0
\end{array}\right]
$$

De forma similar as derivadas $\frac{\partial^{2} \mathrm{q}_{2}}{\partial \mathrm{p}_{\mathrm{i}} \partial \mathrm{p}_{\mathrm{j}}}$ dão origem a outra matriz simétrica 


$$
\mathbf{G}_{2}=\frac{\partial^{2} \mathbf{q}_{2}}{\partial \mathrm{p}_{\mathrm{i}} \partial \mathrm{p}_{\mathrm{j}}}
$$

que explicitada fica:

$$
\mathbf{G}_{2}=\ell_{\mathrm{c}}^{-2}\left[\begin{array}{cccccc}
2 \operatorname{sen} \varphi_{\mathrm{c}} \cos \varphi_{\mathrm{c}} & \cos ^{2} \varphi_{\mathrm{c}}-\operatorname{sen}^{2} \varphi_{\mathrm{c}} & 0 & 2 \operatorname{sen} \varphi_{\mathrm{c}} \cos \varphi_{\mathrm{c}} & \operatorname{sen}^{2} \varphi_{\mathrm{c}}-\cos ^{2} \varphi_{\mathrm{c}} & 0 \\
& 2 \operatorname{sen} \varphi_{\mathrm{c}} \cos \varphi_{\mathrm{c}} & 0 & \operatorname{sen}^{2} \varphi_{\mathrm{c}}-\cos ^{2} \varphi_{\mathrm{c}} & -2 \operatorname{sen} \varphi_{\mathrm{c}} \cos \varphi_{\mathrm{c}} & 0 \\
& & 0 & 0 & 0 & 0 \\
& & & -2 \operatorname{sen} \varphi_{\mathrm{c}} \cos \varphi_{\mathrm{c}} & \cos ^{2} \varphi_{\mathrm{c}}-\operatorname{sen}^{2} \varphi_{\mathrm{c}} & 0 \\
& & & & 2 \operatorname{sen} \varphi_{\mathrm{c}} \cos \varphi_{\mathrm{c}} & 0 \\
& & & & & 0
\end{array}\right]
$$

A seguir mostra-se a obtenção de uma elemento da matriz $\mathbf{G}_{2}$

$$
\mathrm{q}_{2,11}=\frac{\partial^{2} \mathrm{q}_{2}}{\partial \mathrm{p}_{1} \partial \mathrm{p}_{1}}=\frac{\partial}{\partial \mathrm{p}_{1}}\left(\frac{\partial \mathrm{q}_{2}}{\partial \mathrm{p}_{1}}\right)
$$

com auxílio de $(3.60 \mathrm{~g})$, vem:

$$
\begin{aligned}
\mathrm{q}_{2,11} & =\frac{\partial}{\partial \mathrm{p}_{1}}\left(-\frac{\operatorname{sen} \varphi_{\mathrm{c}}}{\ell_{\mathrm{c}}}\right) \\
& =-\frac{\ell_{\mathrm{c}} \cos \varphi_{\mathrm{c}}\left(\partial \varphi_{\mathrm{c}} / \partial \mathrm{p}_{1}\right)-\operatorname{sen} \varphi_{\mathrm{c}}\left(\partial \ell_{\mathrm{c}} / \partial \mathrm{p}_{1}\right)}{\ell_{\mathrm{c}}^{2}} \\
& =-\frac{\ell_{\mathrm{c}} \cos \varphi_{\mathrm{c}}\left(\operatorname{sen} \varphi_{\mathrm{c}} / \ell_{\mathrm{c}}\right)-\operatorname{sen} \varphi_{\mathrm{c}}\left(-\cos \varphi_{\mathrm{c}}\right)}{\ell_{\mathrm{c}}^{2}} \\
\mathrm{q}_{2,11} & =-\frac{2 \operatorname{sen} \varphi_{\mathrm{c}} \cos \varphi_{\mathrm{c}}}{\ell_{\mathrm{c}}^{2}}
\end{aligned}
$$


Da mesma forma as derivadas $\frac{\partial^{2} q_{3}}{\partial p_{i} \partial p_{j}}$ dão origem a matriz simétrica

$$
\mathbf{G}_{3}=\frac{\partial^{2} \mathrm{q}_{3}}{\partial \mathrm{p}_{\mathrm{i}} \partial \mathrm{p}_{\mathrm{j}}}
$$

Das equações (3.60g) a (3.60r) pode-se observar que os elementos não constantes de $\partial \mathrm{q}_{3} / \partial \mathrm{p}_{\mathrm{i}}$ são iguais aos elementos não constantes de $\partial \mathrm{q}_{2} / \partial \mathrm{p}_{\mathrm{i}}$, portanto, pode-se concluir que:

$$
\frac{\partial^{2} q_{3}}{\partial p_{i} \partial p_{j}}=\frac{\partial^{2} q_{2}}{\partial p_{i} \partial p_{j}}
$$

ou seja

$$
\mathbf{G}_{3}=\mathbf{G}_{2}
$$

Estas derivadas $q_{\alpha, i j}$ são relações que envolvem apenas geometria, ou seja, deslocamentos em coordenadas naturais e deslocamentos em coordenadas cartesianas. Portanto, as matrizes $\mathbf{G}_{\alpha}(\alpha=1,3)$ podem ser interpretadas como uma parcela da conhecida "matriz de rigidez geométrica".

As matrizes $\mathbf{G}_{\alpha}$ podem ser escritas como um triplo produto matricial,

$$
\mathbf{G}_{\alpha}=\mathbf{T}^{\mathrm{t}} \overline{\mathbf{G}}_{\boldsymbol{\alpha}} \mathbf{T}
$$

onde $\overline{\mathbf{G}}_{\alpha}$ é a forma local de $\mathbf{G}_{\alpha}$ e $\mathbf{T}$ é a matriz de rotação de eixos dada por (3.65). Logo: 


$$
\begin{aligned}
& \overline{\mathbf{G}}_{1}=\frac{1}{\ell_{\mathrm{c}}}\left[\begin{array}{cccccc}
0 & 0 & 0 & 0 & 0 & 0 \\
& 1 & 0 & 0 & -1 & 0 \\
& & 0 & 0 & 0 & 0 \\
\text { simétrica } & & & 1 & 0 \\
& & & & & 0
\end{array}\right] \\
& \overline{\mathbf{G}}_{2}=\overline{\mathbf{G}}_{3}=\frac{1}{\ell_{\mathrm{c}}^{2}}\left[\begin{array}{cccccc}
0 & 1 & 0 & 0 & -1 & 0 \\
& 0 & 0 & -1 & 0 & 0 \\
& & 0 & 0 & 0 & 0 \\
\text { simétrica } & & & 0 & 0 \\
& & & & &
\end{array}\right]
\end{aligned}
$$

Cabe observar que a matriz $\overline{\mathbf{G}}_{1}$ é a responsável pelo conhecido "efeito P- $\Delta$ ".

\subsection{Teoria Estrutural. Definição dos Campos de Deformações e de Deslocamentos do Elemento}

A teoria estrutural utilizada neste trabalho parte da hipótese cinemática de Euler-Bernoulli-Navier, ou seja:

"As seções transversais inicialmente planas e ortogonais ao eixo da barra permanecem planas, indeformáveis e ortogonais ao eixo, após a deformação".

Consideram-se, portanto, desprezíveis as distorções no plano que contém o eixo da barra, bem como o efeito da deformação transversal ou de Poisson, isto é, sendo yz o plano da seção transversal, tem-se:

$$
\varepsilon_{\mathrm{xx}} \neq 0 ; \quad \varepsilon_{\mathrm{yy}}=0 ; \quad \varepsilon_{\mathrm{zz}}=0 ; \quad \varepsilon_{\mathrm{zy}}=0 ; \quad \text { e } \quad v=0
$$


Da hipótese de que não há distorção nas seções transversais decorre que a única deformação relevante é, portanto, a deformação longitudinal $\varepsilon_{x x}$ a partir de agora, denominada simplesmente " $\varepsilon$ ".

Tomando-se um elemento diferencial de uma barra reta de pórtico plano na configuração inicial ou de referência, como mostrado na Fig. 3.5a, limitado por duas seções transversais ortogonais a um eixo arbitrário e distantes $\mathrm{dx}_{\mathrm{r}}$ uma da outra.

Chamando de fibra a um conjunto de pontos materiais sobre uma reta ou curva paralela ao eixo, verifica-se que uma fibra genérica situada a uma distância $y_{r}$ do eixo e uma fibra neste eixo têm os comprimentos $d s_{r}$ e $d \bar{s}_{r}$ respectivamente, dados por:

$$
\mathrm{ds}_{\mathrm{r}}=\mathrm{ds}_{\mathrm{r}}=\mathrm{dx}
$$

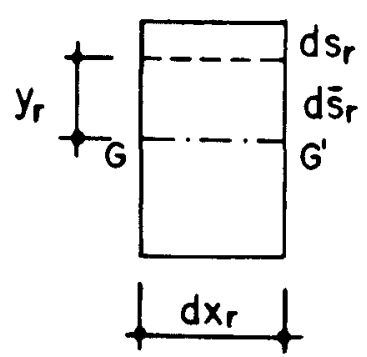

(a) CONFIGURAÇÃO INICIAL OU DE REFERẼNCIA.

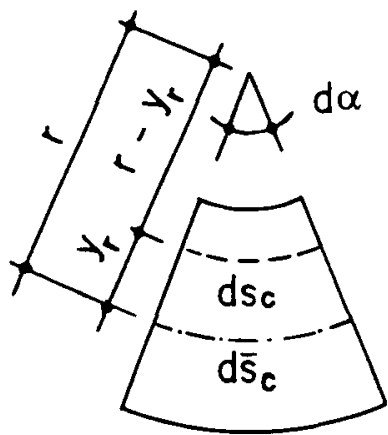

(b) CONFIGURAÇÃO DEFORMADA OU CORRIGIDA.

FIGURA 3.5 Elemento Diferencial de Barra Reta

Seja agora o mesmo elemento diferencial de barra na configuração deformada ou corrigida conforme mostrado na Fig. 3.5b. Pela hipótese de Navier as seções planas permanecerão planas, sendo assim, obtém-se para uma fibra distante $y_{r}$ do eixo e uma fibra no eixo os seguinte comprimentos: 


$$
\begin{aligned}
& d s_{c}=\left(r-y_{r}\right) d \alpha \\
& e \\
& d \bar{s}_{c}=r d \alpha
\end{aligned}
$$

onde $r$ é o raio de curvatura.

De (3.124) e (3.125) tem-se:

$$
\begin{aligned}
& d s_{c}=r d \alpha-y_{r} d \alpha \quad \text { ou } \\
& d s_{c}=d \bar{s}_{c}-y_{r} d \alpha
\end{aligned}
$$

Como visto anteriormente o estiramento $(\lambda)$ de uma fibra situada a uma distância $y_{\mathrm{r}}$ do eixo e o estiramento $(\bar{\lambda})$ de uma fibra no eixo são definidos por:

$$
\lambda=\frac{d s_{c}}{d s_{r}}
$$

e

$$
\bar{\lambda}=\frac{\overline{d s}_{\mathrm{c}}}{\mathrm{ds}_{\mathrm{r}}}
$$

Substituindo (3.126) em (3.127) vem:

$$
\lambda=\frac{\mathrm{ds}_{\mathrm{c}}-\mathrm{y}_{\mathrm{r}} \mathrm{d} \alpha}{\mathrm{ds_{ \textrm {r } }}}
$$


mas, de (3.123) resulta:

$$
\lambda=\frac{\mathrm{d} \bar{s}_{\mathrm{c}}}{\mathrm{ds}_{\mathrm{r}}}-\frac{\mathrm{y}_{\mathrm{r}} \mathrm{d} \alpha}{\mathrm{dx}}
$$

com auxílio de (3.128) obtém-se:

$$
\lambda=\bar{\lambda}-\mathrm{y}_{\mathrm{r}} \alpha^{\prime}
$$

onde ' representa a derivação em relação a $\mathbf{x}_{\mathbf{r}}$.

Adotando como medida de deformação a deformação linear ou de engenharia $(\varepsilon)$, tem-se:

$$
\begin{aligned}
& \bar{\varepsilon}=\bar{\lambda}-1 \\
& \varepsilon=\lambda-1
\end{aligned}
$$

onde $\bar{\varepsilon}$ é a deformação de uma fibra no eixo e $\varepsilon$ é a deformação de uma fibra genérica distante $\mathrm{y}_{\mathrm{r}}$ do eixo, sendo os valores negativos correspondentes a encurtamentos e positivos a alongamentos.

Levando (3.129) em (3.131) vem:

$$
\varepsilon=\bar{\lambda}-\mathrm{y}_{\mathrm{r}} \alpha^{\prime}-1,
$$

com o auxilio de (3.130) resulta:

$$
\varepsilon=\bar{\varepsilon}-\mathrm{y}_{\mathrm{r}} \alpha^{\prime}
$$

que é a expressão do campo de deformação consistente com a teoria estrutural adotada neste trabalho. 
PIMENTA \& MAZZILLI (1986) ressaltam que a rotação específica $\alpha^{\prime}$ é o deslocamento generalizado energeticamente conjugado com o momento fletor. Esta rotação específica $\alpha^{\prime}$ só é igual à curvatura do eixo deformado quando se adota a hipótese de inextensibilidade do eixo da barra (ver eq. 3.134). A curvatura $k$ é definida como:

$$
\mathrm{k}=\frac{1}{\mathrm{r}}=\frac{\mathrm{d} \alpha}{\mathrm{d} \bar{s}_{\mathrm{c}}}
$$

aplicando a regra da cadeia vem:

$$
\mathrm{k}=\frac{1}{\mathrm{r}}=\frac{\mathrm{d} \alpha}{\mathrm{dx}} \frac{\mathrm{dx_{ \textrm {r } }}}{\mathrm{ds}_{\mathrm{c}}}
$$

com o auxílio da eq. (3.128) resulta:

$$
\mathbf{k}=\alpha^{\prime} \frac{1}{\bar{\lambda}}=\frac{\alpha^{\prime}}{\bar{\lambda}}
$$

Se o eixo neutro é inextensível então $\bar{\lambda}=1$ e $k=\alpha^{\prime}$.

Com o objetivo de expressar o campo de deformações em função dos deslocamentos do eixo da barra, serão feitas a seguir algumas considerações analíticas sobre o campo de deslocamentos.

Admitida a hipótese de Navier, pode-se obter os campos de deslocamentos axiais $(\overline{\mathrm{u}})$ e transversais $(\overline{\mathrm{v}})$ do pontos situados sobre o eixo, bem como da rotação $(\alpha)$ das seções transversais (Fig. 3.6).

Os deslocamentos de um ponto genérico $P$, caracterizado pela coordenada $y_{r}$ relativa ao eixo, são dados por: 


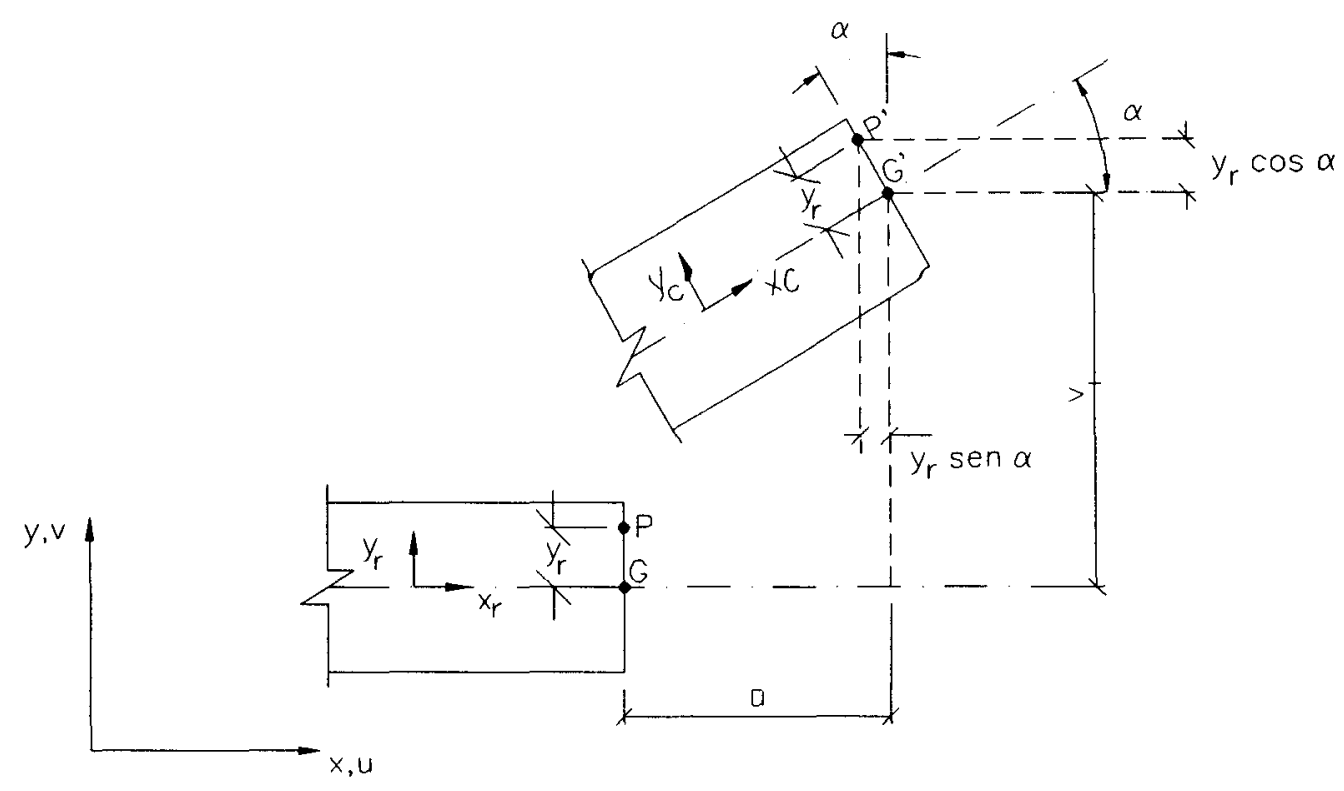

FIGURA 3.6 - Deslocamentos de pontos da barra

$$
\begin{aligned}
& \mathrm{u}=\overline{\mathrm{u}}-\mathrm{y}_{\mathrm{r}} \operatorname{sen} \alpha \\
& \mathrm{v}=\overline{\mathrm{v}}-\mathrm{y}_{\mathrm{r}}(1-\cos \alpha)
\end{aligned}
$$

Estes deslocamentos podem ser escritos no sistema corrotacional ( $\mathbf{x}_{\mathfrak{c}}$, $y_{c}$ ) centrado no eixo do elemento na configuração deformada, como:

$$
\begin{aligned}
& \mathrm{u}_{\mathrm{c}}=\overline{\mathrm{u}}_{\mathrm{c}}-\mathrm{y}_{\mathrm{r}} \operatorname{sen} \alpha \\
& \mathrm{v}_{\mathrm{c}}=\overline{\mathrm{v}}_{\mathrm{c}}-\mathrm{y}_{\mathrm{r}}(1-\cos \alpha)
\end{aligned}
$$

A Fig. 3.7 mostra um elemento diferencial do eixo da barras nas configurações de referência (AB) e deformada ( $\left.A^{\prime} B^{\prime}\right)$. 


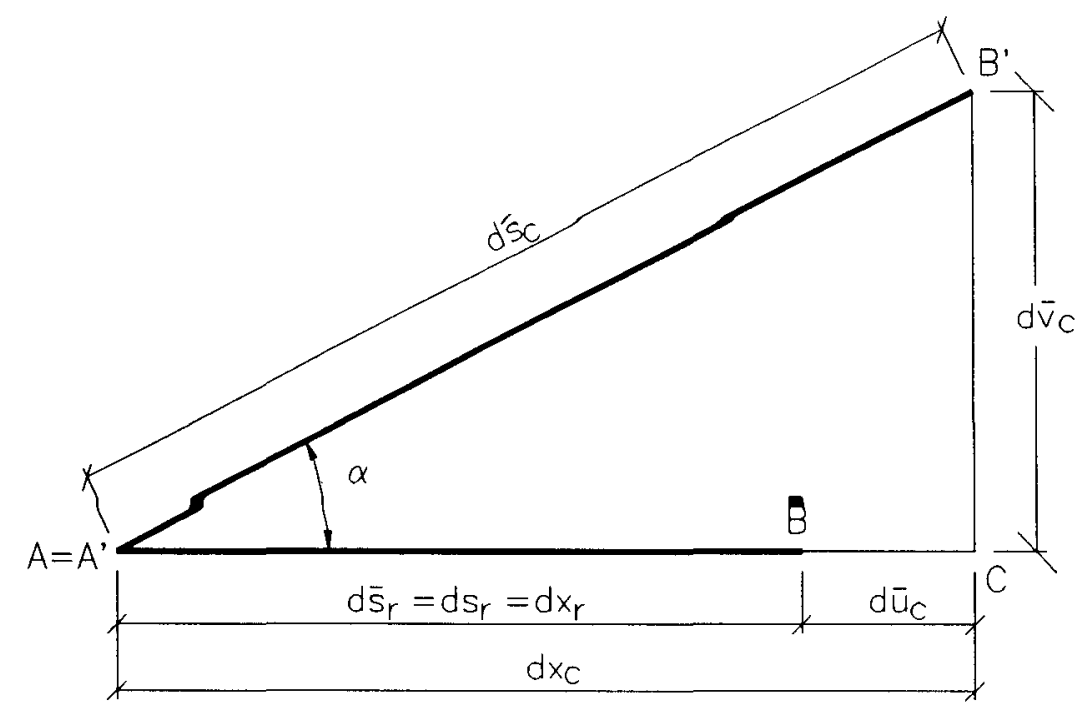

FIGURA 3.7 - Elemento diferencial do eixo da barra nas configurações de referência e deformada

Da Fig. (3.7) tem-se:

$$
\begin{aligned}
& \mathrm{d} \bar{s}_{\mathrm{c}}=\left[\left(d \mathrm{x}_{\mathrm{r}}+\mathrm{d} \overline{\mathrm{u}}_{\mathrm{c}}\right)^{2}+\mathrm{d} \overline{\mathrm{v}}_{\mathrm{c}}{ }^{1 / 2}\right]^{1 / 2} \\
& \operatorname{tg} \alpha=\frac{\mathrm{d} \overline{\mathrm{v}}_{\mathrm{c}}}{\mathrm{dx_{ \textrm {r } }}+\mathrm{d} \overline{\mathrm{u}}_{\mathrm{c}}}=\frac{\frac{\mathrm{d} \overline{\mathrm{v}}_{\mathrm{c}}}{\mathrm{dx_{ \textrm {r } }}}}{\frac{\mathrm{dx_{ \textrm {r } }}}{\mathrm{dx_{ \textrm {r } }}}+\frac{\mathrm{du} \overline{\mathrm{u}}_{\mathrm{c}}}{\mathrm{dx_{ \textrm {r } }}}} \\
& \operatorname{tg} \alpha=\frac{\mathrm{v}_{\mathrm{c}}^{\prime}}{1+\overline{\mathrm{u}}_{\mathrm{c}}^{\prime}} \\
& \mathrm{d \overline {x } _ { \mathrm { c } }}=\mathrm{dx_{ \textrm {r } }}+\mathrm{d} \overline{\mathrm{u}}_{\mathrm{c}}
\end{aligned}
$$

Conforme mostrado anteriormente, o estiramento da fibra do eixo é dado por $\bar{\lambda}=\mathrm{d} \overline{\mathrm{s}}_{\mathrm{c}} / \mathrm{d} \overline{\mathrm{s}}_{\mathrm{r}}$ ou seja: 


$$
\bar{\lambda}=\frac{\mathrm{ds}_{\mathrm{c}}}{\mathrm{dx}_{\mathrm{r}}} \frac{\mathrm{dx_{ \textrm {r } }}}{\mathrm{ds}_{\mathrm{r}}}
$$

E o estiramento da fibra distante $y_{r}$ do eixo é dado por $\lambda=d x_{c} / d x_{r}$. Ainda com a ajuda da Fig. 3.7 tem-se:

$$
\begin{aligned}
& \cos \alpha=\frac{d x_{c}}{d s_{c}}=\frac{d x_{c}}{\bar{\lambda} \frac{-}{d s_{r}}}=\frac{d x_{c}}{\bar{\lambda} d x_{r}} \quad \text { ou } \\
& \cos \alpha=\frac{1}{\bar{\lambda}} \frac{d x_{c}}{d x_{r}} \quad \text { ou } \\
& \cos \alpha=\frac{1}{\bar{\lambda}} \lambda, \quad \text { resultando: } \\
& \bar{\lambda}=\lambda \sec \alpha
\end{aligned}
$$

Levando (3.130) em (3.132) resulta:

$$
\begin{aligned}
& \varepsilon=(\bar{\lambda}-1)-\mathrm{y}_{\mathrm{r}} \alpha^{\prime}, \text { com auxílio de (3.147) tem-se: } \\
& \varepsilon=\lambda \sec \alpha-1-\mathrm{y}_{\mathrm{r}} \alpha^{\prime}, \text { usando (3.148) obtém-se: }
\end{aligned}
$$




$$
\varepsilon=\left(1+\overline{\mathrm{u}}_{\mathrm{c}}^{\prime}\right) \sec \alpha-1-\mathrm{y}_{\mathrm{r}} \alpha^{\prime}
$$

que é a expressão do campo de deformação em função dos deslocamentos u $\mathrm{e}$ $\bar{v}$ do eixo da barra, que podem ser interpolados em função dos graus de liberdade naturais $\mathrm{q}_{1}, \mathrm{q}_{2}$ e $\mathrm{q}_{3}$, resultando portanto, uma expressão do campo de deformações em função apenas dos deslocamentos nodais.

\subsection{Formulação do Equilíbrio Elemental}

Como mostrado no item anterior pode-se expressar o campo de deformações $\varepsilon$, em função dos graus de liberdade naturais $q_{\alpha}(\alpha=1,3)$, que por sua vez, podem ser colocados em função dos graus de liberdade cartesianos $p_{i}$ $(i=1,6)$. Sendo assim, pode-se escrever:

$$
\varepsilon=\varepsilon\left(\mathrm{q}_{\alpha}\left(\mathrm{p}_{\mathrm{i}}\right)\right)
$$

Utilizando-se o Princípio dos Trabalhos Virtuais pode, então ser formulado o equilíbrio do elemento. Seja $\mathrm{P}_{\mathrm{i}}$ o vetor de esforços nodais internos do elemento, $\delta \varepsilon$ a deformação virtual de uma fibra genérica e $\sigma$ a tensão normal na seção transversal, isto é, a tensão na fibra genérica, e $\delta \mathrm{p}_{\mathrm{i}} \mathrm{o}$ vetor dos deslocamentos virtuais dos pontos nodais do elemento. Então o trabalho virtual interno do elemento, em função das tensões, é dado por:

$$
\delta \mathrm{W}_{\mathrm{i}}=\int_{\mathrm{V}_{r}} \sigma \delta \varepsilon d \mathrm{~V}_{\mathrm{r}}
$$

ou ainda, 


$$
\delta \mathrm{W}_{\mathrm{i}}=\int_{-\ell_{\mathrm{r}} / 2}^{\ell_{\mathrm{r}} / 2} \int_{\mathrm{A}_{\mathrm{r}}} \sigma \delta \varepsilon \mathrm{dA}_{\mathrm{r}} \mathrm{dx} \mathrm{x}_{\mathrm{r}}
$$

Em função dos esforços nodais tem-se:

$$
\delta \mathrm{W}_{\mathrm{i}}=\mathrm{P}_{\mathrm{i}} \delta \mathrm{p}_{\mathrm{i}}
$$

onde o índice repetido indica somatório.

A deformação virtual é dada pela variação da equação (3.150), obtendose com o emprego da regra da cadeia:

$$
\delta \varepsilon=\varepsilon_{, \alpha} q_{\alpha, i} \delta p_{i}
$$

onde $\varepsilon_{, \alpha}$ indica derivada de $\varepsilon$ em relação a $q_{\alpha}$ e $q_{\alpha, i}$ indica derivada de $q_{\alpha}$ em relação a $p_{i}$.

Levando-se (3.154) em (3.152) obtém-se:

$$
\int_{-\ell_{\mathrm{r}} / 2}^{\ell_{\mathrm{r}} / 2} \int_{A_{\mathrm{r}}} \sigma \varepsilon_{, \alpha} \mathrm{q}_{\alpha, \mathrm{i}} \delta \mathrm{p}_{\mathrm{i}} \mathrm{dA} \mathrm{A}_{\mathrm{r}} \mathrm{dx} \mathrm{x}_{\mathrm{r}}=\mathrm{P}_{\mathrm{i}} \delta \mathrm{p}_{\mathrm{i}}
$$

que é válida para qualquer deslocamento virtual $\delta \mathrm{p}_{\mathrm{i}}$, resultando assim:

$$
P_{i}=\int_{-_{r} / 2}^{\ell_{r} / 2} \int_{A_{r}} \sigma \varepsilon_{, \alpha} q_{\alpha, i} d A_{r} d x_{r}
$$

que é a equação de equilíbrio do elemento na direção da função deslocamento virtual.

Como a derivada $q_{\alpha, i}$ não depende de $\mathrm{dA}_{\mathrm{r}} \mathrm{dx}_{\mathrm{r}}$, este termo pode ser colocado para fora da integral, resultando então: 


$$
P_{i}=\left(\int_{-\ell_{r} / 2}^{\ell_{r} / 2} \int_{A_{r}} \sigma \varepsilon_{, \alpha} d A_{r} d x_{r}\right) q_{\alpha, i}
$$

onde $q_{\alpha, i}$ é a matriz B dada pela equação (3.60).

Chamando de $\mathrm{Q}_{\alpha}$ os esforços internos em coordenadas naturais energeticamente conjugados com os deslocamentos $q_{\alpha}$ em coordenadas naturais, pode-se escrever o trabalho virtual interno como:

$$
\delta \mathrm{W}_{\mathrm{i}}=\mathrm{Q}_{\alpha} \delta \mathrm{q}
$$

De (3.60) sabe-se que os deslocamentos virtuais em coordenadas naturais $\delta q$ estão relacionados com os deslocamentos virtuais cartesianos $\delta p$ através da matriz $q_{\alpha, i}$, ou seja

$$
\delta q=q_{\alpha, i} \delta p
$$

levando (3.159) em (3.158) tem-se:

$$
\delta \mathrm{W}_{\mathrm{i}}=\mathrm{Q}_{\alpha} \mathrm{q}_{\alpha, \mathrm{i}} \delta \mathrm{p}
$$

Igualando as equações (3.155) e (3.160), obtém-se:

$$
\mathrm{Q}_{\alpha} \mathbf{q}_{\alpha, i} \delta \mathrm{p}=\int_{-t_{\mathrm{r}} / 2}^{t_{\mathrm{r}} / 2} \int_{\mathrm{A}_{\mathrm{r}}} \sigma \varepsilon_{, \alpha} \mathrm{q}_{\alpha, i} \delta \mathrm{pdA_{ \textrm {r } }} \mathrm{dx}_{\mathrm{r}}
$$

resultando:

$$
\mathrm{Q}_{\alpha}=\int_{-\mathrm{l}_{\mathrm{r}} / 2}^{\mathrm{I}_{\mathrm{r}} / 2} \int_{\mathrm{A}_{\mathrm{r}}} \sigma \varepsilon_{, \alpha} \mathrm{dA_{ \textrm {r } }} \mathrm{dx}
$$


Das equações (3.157) e (3.161) obtém-se a relação entre os esforços nodais naturais " $\mathrm{Q}_{\alpha}$ " e os esforços nodais cartesianos " $\mathrm{P}_{\mathrm{i}}$ ", dada por:

$$
\mathrm{P}_{\mathrm{i}}=\mathrm{Q}_{\alpha} \mathrm{q}_{\alpha, \mathrm{i}}
$$

onde $\mathrm{o}$ indice repetido indica somatório.

Em notação matricial, tem-se:

$$
\mathbf{P}=\mathbf{B}^{\mathrm{t}} \mathbf{Q}
$$

\subsection{Matriz de Rigidez Tangente do Elemento}

A matriz de rigidez tangente do elemento, no sistema de coordenadasdeslocamento cartesiano, é dada por:

$$
\mathbf{k}=\frac{\partial \mathbf{P}_{\mathrm{i}}}{\partial \mathbf{p}_{\mathrm{j}}}
$$

ou seja, os elementos de rigidez tangente $\mathrm{k}_{\mathrm{ij}}$ são obtidos da derivação parcial de $P_{\mathrm{i}}$ em relação aos deslocamentos nodais cartesianos $\mathrm{p}_{\mathrm{j}}$.

Analogamente, a matriz de rigidez tangente, em coordenadasdeslocamento naturais é dada por:

$$
\mathbf{k}^{*}=\frac{\partial \mathrm{Q}_{\alpha}}{\partial \mathrm{q}_{\beta}}
$$

ou seja, os elementos de rigidez $k_{\alpha \beta}^{*}$ são obtidos da derivação parcial de $Q_{\alpha}$ em relação aos deslocamentos nodais naturais $\mathrm{q}_{\beta}$. 
A partir da equação (3.162) e utilizando a regra da cadeia obtém-se:

$$
\mathbf{k}_{\mathrm{ij}}=\mathrm{Q}_{\alpha} \mathbf{q}_{\alpha, \mathrm{j}}+\mathrm{q}_{\alpha, \mathrm{i}}\left(\mathrm{Q}_{\alpha, \beta} \mathrm{q}_{\beta, \mathrm{j}}\right)
$$

Derivando a equação (3.161), obtém-se:

$$
\mathrm{Q}_{\alpha, \beta}=\int_{-\ell_{\mathrm{r}} / 2}^{\ell_{\mathrm{r}} / 2} \int_{\mathrm{A}_{\mathrm{r}}} \sigma \varepsilon_{, \alpha \beta} \mathrm{dA}_{\mathrm{r}} \mathrm{dx}_{\mathrm{r}}+\int_{-\ell_{\mathrm{r}} / 2}^{\ell_{\mathrm{r}} / 2} \int_{\mathrm{A}_{\mathrm{r}}}\left(\frac{\partial \sigma}{\partial \varepsilon} \varepsilon_{\beta}\right) \varepsilon_{, \alpha} \mathrm{dA}_{\mathrm{r}} \mathrm{d} \mathrm{x}_{\mathrm{r}}
$$

ou com o auxílio de (3.35)

$$
Q_{\alpha, \beta}=\int_{-t_{r} / 2}^{t_{r} / 2} \int_{A_{r}} \sigma \varepsilon_{, \alpha \beta} d A_{r} d x_{r}+\int_{-t_{r} / 2}^{t_{r} / 2} \int_{A_{r}} D \varepsilon_{, \beta} \varepsilon_{, \alpha} d A_{r} d x_{r}
$$

Fazendo:

$$
H_{\alpha \beta}=\int_{-\ell_{r} / 2}^{\ell_{r} / 2} \int_{A_{r}} \sigma \varepsilon_{, \alpha \beta} d A_{r} d x_{r}
$$

e

$$
D_{\alpha \beta}=\int_{-\ell_{r} / 2}^{l_{1} / 2} \int_{A_{r}} D \varepsilon_{\beta, \beta} \varepsilon_{, \alpha} d A_{r} d x_{r}
$$

resulta:

$$
\mathbf{k}_{\alpha \beta}^{*}=Q_{\alpha, \beta}=H_{\alpha \beta}+D_{\alpha \beta}
$$

A parcela $\mathrm{H}_{\alpha \beta}$ foi obtida fixando-se a tensão e variando-se a geometria e, representa a conhecida matriz de rigidez geométrica em coordenadas naturais.

A parcela $D_{\alpha \beta}$, obtida fixando-se a geometria e variando-se a tensão, representa a matriz de rigidez constitutiva em coordenadas naturais. 
Como mostrado anteriormente (equações $3.111,3.113$ e 3.116 ), a derivada $q_{\alpha, j}=G_{\alpha}$, assim, a expressão dos elementos da matriz de rigidez tangente em coordenadas cartesianas (eq. 3.166) fica:

$$
\mathbf{k}_{\mathrm{ij}}=\mathrm{q}_{\alpha, \mathrm{i}}\left(\mathrm{H}_{\alpha \beta}+\mathrm{D}_{\alpha \beta}\right) \mathrm{q}_{\beta, \mathrm{j}}+\mathrm{Q}_{\alpha} \mathrm{G}_{\alpha}
$$

com o auxílio de (3.170) vem:

$$
\mathbf{k}_{\mathrm{ij}}=\mathbf{q}_{\alpha, \mathrm{i}} \mathbf{k}_{\alpha \beta}^{*} \mathrm{q}_{\beta, \mathrm{j}}+\mathrm{Q}_{\alpha} \mathrm{G}_{\alpha}
$$

onde $\mathrm{o}$ índice repetido indica somatório.

O primeiro termo representa a usual transformação de matriz de rigidez de um sistema fixo de coordenadas para outro também fixo. O segundo termo corresponde a fixar as forças nodais $\mathrm{Q}_{\alpha}$ e derivar a relação entre os deslocamentos nodais cartesianos e naturais, levando-se em consideração o fato do sistema de coordenadas corrotacional variar com os deslocamentos globais cartesianos, ou seja, da matriz de mudança de coordenadas não ser constante.

A equação (3.171) na forma matricial fica:

$$
\mathbf{k}=\mathbf{B}^{\mathrm{t}} \mathbf{D} \mathbf{B}+\mathbf{B}^{\mathbf{t}} \mathbf{H} \mathbf{B}+\mathrm{Q}_{\alpha} \mathbf{G}_{\alpha}
$$

ou

$$
\mathbf{k}=\mathbf{k}_{\mathrm{M}}+\mathbf{k}_{\mathrm{G}}
$$

onde

$$
\begin{aligned}
& \mathbf{k}_{\mathbf{M}}=\mathbf{B}^{\mathrm{t}} \mathbf{D} \mathbf{B} \\
& \mathbf{k}_{\mathbf{G}}=\mathbf{B}^{\mathrm{t}} \mathbf{H} \mathbf{B}+\mathbf{Q}_{\alpha} \mathbf{G}_{\alpha}
\end{aligned}
$$


sendo

$$
\mathrm{Q}_{\alpha} \mathbf{G}_{\alpha}=\mathrm{Q}_{1} \mathbf{G}_{1}+\mathrm{Q}_{2} \mathbf{G}_{2}+\mathrm{Q}_{3} \mathbf{G}_{3}
$$

$\mathbf{k}_{\mathrm{M}}$ é a chamada matriz de rigidez constitutiva do elemento, que depende do módulo de rigidez tangente do material dado pela equação (3.35).

$\mathbf{k}_{\mathrm{G}}$ é a chamada matriz de rigidez geométrica do elemento, que depende da geometría e do nível de tensão.

\subsection{Funções de Interpolação dos Deslocamentos}

Com o objetivo de obter as integrais $Q_{\alpha}, D_{\alpha \beta}$ e $H_{\alpha \beta}$, que definem completamente as equações de equilíbrio e a matriz de rigidez do elemento de barra do pórtico plano, serão introduzidas funções aproximadoras para os deslocamentos $\bar{u}$ e $\bar{v}$ do eixo do elemento.

Seja o elemento de barra, mostrado na Fig. 3.8 em sua configuração de referência $A B$ e deformada $A^{\prime} B^{\prime}$.

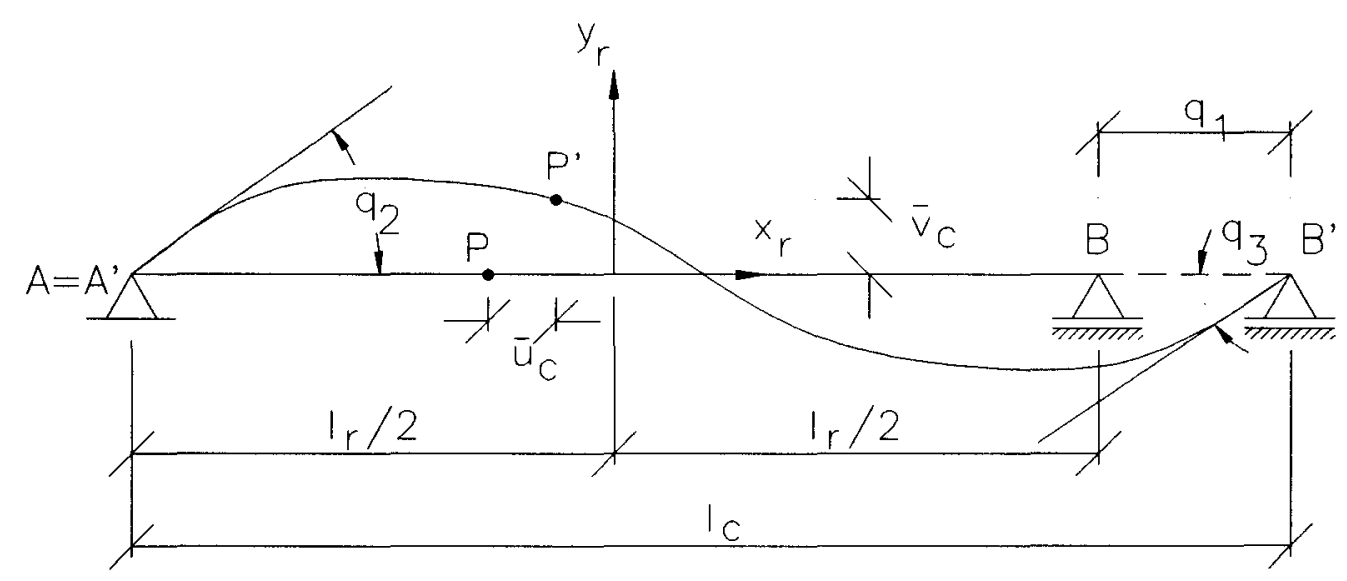

FIGURA 3.8 - Elemento de barra de pórtico plano

Os deslocamentos axiais $\bar{u}_{c}$ serão interpolados linearmente, conforme adotado por MAZZILLI (1989), ou seja: 


$$
\overline{\mathrm{u}}_{\mathrm{c}}=\mathrm{a} \mathrm{x}_{\mathrm{r}}+\mathrm{b}
$$

As grandezas a e b podem se obtidas particularizando-se $\bar{u}_{c}$ nas extremidades do elemento, ou seja:

$$
\begin{gathered}
\mathrm{x}_{\mathrm{r}}=-\frac{\ell_{\mathrm{r}}}{2} \Rightarrow \overline{\mathrm{u}}_{\mathrm{c}}=0 \Rightarrow 0=\mathrm{a} \frac{\ell_{\mathrm{r}}}{2}+\mathrm{b} \Rightarrow \mathrm{b}=\frac{\mathrm{a} \ell_{\mathrm{r}}}{2} \\
\mathrm{x}_{\mathrm{r}}=\frac{\ell_{\mathrm{r}}}{2} \Rightarrow \overline{\mathrm{u}}_{\mathrm{c}}=\mathrm{q}_{1} \Rightarrow \mathrm{q}_{1}=\mathrm{a} \frac{\ell_{\mathrm{r}}}{2}+\mathrm{b} \Rightarrow \mathrm{q}_{1}=\frac{\mathrm{a} \ell_{\mathrm{r}}}{2}+\frac{\mathrm{a} \ell_{\mathrm{r}}}{2} \Rightarrow \mathrm{a}=\frac{\mathrm{q}_{1}}{\ell_{\mathrm{r}}} \\
\mathrm{b}=\frac{\mathrm{q}_{1} \ell_{\mathrm{r}}}{2 \ell_{\mathrm{r}}} \Rightarrow \mathrm{b}=\frac{\mathrm{q}_{1}}{2}
\end{gathered}
$$

então a função interpoladora de $\overline{\mathbf{u}}_{c}$ em termos dos deslocamentos nodais naturais será:

$$
\bar{u}_{\mathrm{c}}=\mathrm{q}_{1}\left(\frac{1}{2}+\frac{\mathrm{x}_{\mathrm{r}}}{\ell_{\mathrm{r}}}\right)
$$

A derivada de $\bar{u}_{c}$ em relação a $x_{r}$ será:

$$
\overline{\mathbf{u}}_{\mathrm{c}}^{\prime}=\frac{\mathrm{q}_{1}}{\ell_{\mathrm{r}}}
$$

Os deslocamentos transversais $\bar{v}_{c}$ serão interpolados cubicamente pela função:

$$
\bar{v}_{c}=c x_{r}^{3}+d x_{r}^{2}+e x_{r}+f
$$


Analogamente as grandezas c, d, e, f podem ser obtidas particularizando-se $\bar{v}_{c}$ e $\bar{v}_{c}^{\prime}$ nos extremos, ou seja:

$$
\begin{aligned}
& \mathrm{x}_{\mathrm{r}}=-\frac{\ell_{\mathrm{r}}}{2} \Rightarrow \overline{\mathrm{v}}_{\mathrm{c}}=0 \Rightarrow 0=-\mathrm{c} \frac{\ell_{\mathrm{r}}^{3}}{8}+\mathrm{d} \frac{\ell_{\mathrm{r}}^{2}}{4}-\mathrm{e} \frac{\ell_{\mathrm{r}}}{2}+\mathrm{f} \\
& \mathrm{x}_{\mathrm{r}}=\frac{\ell_{\mathrm{r}}}{2} \Rightarrow \overline{\mathrm{v}}_{\mathrm{c}}=0 \Rightarrow 0=\mathrm{c} \frac{\ell_{\mathrm{r}}^{3}}{8}+\mathrm{d} \frac{\ell_{\mathrm{r}}^{2}}{4}+\mathrm{e} \frac{\ell_{\mathrm{r}}}{2}+\mathrm{f}
\end{aligned}
$$

derivando-se $\bar{v}_{c}$ em relação a $x_{r}$ tem-se:

$$
\bar{v}_{c}^{\prime}=3 c x_{r}^{2}+2 d x_{r}+e
$$

nas extremidades tem-se:

$$
\begin{gathered}
\mathrm{x}_{\mathrm{r}}=-\frac{\ell_{\mathrm{r}}}{2} \Rightarrow \operatorname{tg} \alpha=\operatorname{tg} \mathrm{q}_{2}, \text { ou com o auxílio de (3.143) } \\
\overline{\mathrm{v}}_{\mathrm{c}}^{\prime}=\left(1+\overline{\mathrm{u}}_{\mathrm{c}}^{\prime}\right) \operatorname{tg} \mathrm{q}_{2}=\left(1+\frac{\mathrm{q}_{1}}{\ell_{\mathrm{r}}}\right) \operatorname{tg} \mathrm{q}_{2} \Rightarrow \\
\left(1+\frac{\mathrm{q}_{1}}{\ell_{\mathrm{r}}}\right) \operatorname{tg} \mathrm{q}_{2}=3 \mathrm{c} \frac{\ell_{\mathrm{r}}^{2}}{4}+2 \mathrm{~d}\left(-\frac{\ell_{\mathrm{r}}}{2}\right)+\mathrm{e} \Rightarrow \\
\left(\ell_{\mathrm{r}}+\mathrm{q}_{1}\right) \operatorname{tg} \mathrm{q}_{2}=3 \mathrm{c} \frac{\ell_{\mathrm{r}}^{3}}{4}-\mathrm{d} \ell_{\mathrm{r}}^{2}+\mathrm{e} \ell_{\mathrm{r}} \\
\mathrm{x}_{\mathrm{r}}=\frac{\ell_{\mathrm{r}}}{2} \Rightarrow \operatorname{tg} \alpha=\operatorname{tg} \mathrm{q}_{3}, \text { ou com o auxílio de (3.143) }
\end{gathered}
$$




$$
\begin{aligned}
& {\overline{\mathrm{v}_{\mathrm{c}}}}^{\prime}=\left(1+\overline{\mathrm{u}}_{\mathrm{c}}^{\prime}\right) \operatorname{tg} \mathrm{q}_{3}=\left(1+\frac{\mathrm{q}_{1}}{\ell_{\mathrm{r}}}\right) \operatorname{tg} \mathrm{q}_{3} \Rightarrow \\
& \left(1+\frac{\mathrm{q}_{1}}{\ell_{\mathrm{r}}}\right) \operatorname{tg} \mathrm{q}_{3}=3 \mathrm{c} \frac{\ell_{\mathrm{r}}^{2}}{4}+2 \mathrm{~d} \frac{\ell_{\mathrm{r}}}{2}+\mathrm{e} \Rightarrow \\
& \left(\ell_{\mathrm{r}}+\mathrm{q}_{1}\right) \operatorname{tg} \mathrm{q}_{3}=3 \mathrm{c} \frac{\ell_{\mathrm{r}}^{3}}{4}+\mathrm{d} \ell_{\mathrm{r}}^{2}+\mathrm{e} \ell_{\mathrm{r}}
\end{aligned}
$$

fazendo-se (4) - (3) tem-se:

$$
\begin{aligned}
& \left(\ell_{\mathrm{r}}+\mathrm{q}_{1}\right)\left(\operatorname{tg} \mathrm{q}_{3}-\operatorname{tg} \mathrm{q}_{2}\right)=2 \mathrm{~d} \ell_{\mathrm{r}}^{2} \Rightarrow \\
& \mathrm{d}=\frac{\ell_{\mathrm{r}}+\mathrm{q}_{1}}{2 \ell_{\mathrm{r}}^{2}}\left(\operatorname{tg} \mathrm{q}_{3}-\operatorname{tg} \mathrm{q}_{2}\right)
\end{aligned}
$$

somando-se (1) + (2) resulta:

$$
\begin{gathered}
2 \mathrm{f}=-\frac{\mathrm{d} \ell_{\mathrm{r}}^{2}}{2} \Rightarrow \mathrm{f}=-\frac{\mathrm{d} \ell_{\mathrm{r}}^{2}}{4} \Rightarrow \mathrm{f}=\frac{\ell_{\mathrm{r}}+\mathrm{q}_{1}}{2 \ell_{\mathrm{r}}^{2}}\left(\operatorname{tg} \mathrm{q}_{3}-\operatorname{tg} \mathrm{q}_{2}\right)\left(-\frac{1}{2} \ell_{\mathrm{r}}^{2}\right) \Rightarrow \\
\mathrm{f}=-\left(\frac{\mathrm{q}_{1}+\ell_{\mathrm{r}}}{8}\right)\left(\operatorname{tg} \mathrm{q}_{3}-\operatorname{tg} \mathrm{q}_{2}\right)
\end{gathered}
$$

fazendo-se (2) - (1), vem:

$$
\frac{\mathrm{c} \ell_{\mathrm{r}}^{3}}{4}+\mathrm{e} \ell_{\mathrm{r}}=0 \Rightarrow \mathrm{e}=-\frac{\mathrm{c} \ell_{\mathrm{r}}^{2}}{4}
$$


somando-se (3) + (4), vem:

$$
6 \frac{\mathrm{c} \ell_{\mathrm{r}}^{3}}{4}+2 \mathrm{e} \ell_{\mathrm{r}}=\left(\ell_{\mathrm{r}}+\mathrm{q}_{1}\right)\left(\operatorname{tg} \mathrm{q}_{2}+\operatorname{tg} \mathrm{q}_{3}\right) \text {, com o auxílio de (5), }
$$

resulta:

$$
\begin{aligned}
& 6 \frac{c \ell_{\mathrm{r}}^{3}}{4}-2 \frac{c \ell_{\mathrm{r}}^{3}}{4}=\left(\ell_{\mathrm{r}}+\mathrm{q}_{1}\right)\left(\operatorname{tg} \mathrm{q}_{2}+\operatorname{tg} \mathrm{q}_{3}\right) \quad \text { ou } \\
& \mathrm{c}=\frac{1}{\ell_{\mathrm{r}}^{3}}\left(\ell_{\mathrm{r}}+\mathrm{q}_{1}\right)\left(\operatorname{tg} \mathrm{q}_{2}+\operatorname{tg} \mathrm{q}_{3}\right) \\
& \mathrm{e}=-\frac{1}{4 \ell_{\mathrm{r}}}\left(\ell_{\mathrm{r}}+\mathrm{q}_{1}\right)\left(\operatorname{tg} \mathrm{q}_{2}+\operatorname{tg} \mathrm{q}_{3}\right)
\end{aligned}
$$

Então a função interpoladora de $\bar{v}_{\mathrm{c}}$ em termos dos deslocamentos nodais naturais fica:

$$
\begin{aligned}
& \bar{v}_{\mathrm{c}}=\left(1+\frac{\mathrm{q}_{1}}{\ell_{\mathrm{r}}}\right)\left\{\frac{\mathrm{x}_{\mathrm{r}}^{3}}{\ell_{\mathrm{r}}^{2}}\left[\left(\operatorname{tg} \mathrm{q}_{2}+\operatorname{tg} \mathrm{q}_{3}\right)\right]+\frac{\mathrm{x}_{\mathrm{r}}^{2}}{2 \ell_{\mathrm{r}}}\left[\left(\operatorname{tg} \mathrm{q}_{3}-\operatorname{tg} \mathrm{q}_{2}\right)\right]\right. \\
& \left.-\frac{\mathrm{x}_{\mathrm{r}}}{4}\left[\left(\operatorname{tg} \mathrm{q}_{2}+\operatorname{tg} \mathrm{q}_{3}\right)\right]-\frac{\ell_{\mathrm{r}}}{8}\left[\left(\operatorname{tg} \mathrm{q}_{3}-\operatorname{tg} \mathrm{q}_{2}\right)\right]\right\} \text { ou } \\
& \bar{v}_{\mathrm{c}}=\left(1+\frac{\mathrm{q}_{1}}{\ell_{\mathrm{r}}}\right)\left\{\left[\left(\operatorname{tg} \mathrm{q}_{2}+\operatorname{tg} \mathrm{q}_{3}\right)\left(\frac{\mathrm{x}_{\mathrm{r}}^{3}}{\ell_{\mathrm{r}}^{2}}-\frac{\mathrm{x}_{\mathrm{r}}}{4}\right)\right]\right. \\
& \left.+\left[\left(\operatorname{tg} \mathrm{q}_{3}-\operatorname{tg} \mathrm{q}_{2}\right)\left(\frac{\mathrm{x}_{\mathrm{r}}^{2}}{2 \ell_{\mathrm{r}}}-\frac{\ell_{\mathrm{r}}}{8}\right)\right]\right\} \text { ou }
\end{aligned}
$$




$$
\begin{aligned}
& \bar{v}_{\mathrm{c}}=\left(1+\frac{\mathrm{q}_{1}}{\ell_{\mathrm{r}}}\right) {\left[\operatorname{tg} \mathrm{q}_{2}\left(\frac{\mathrm{x}_{\mathrm{r}}^{3}}{\ell_{\mathrm{r}}^{2}}-\frac{\mathrm{x}_{\mathrm{r}}^{2}}{2 \ell_{\mathrm{r}}}-\frac{\mathrm{x}_{\mathrm{r}}}{4}+\frac{\ell_{\mathrm{r}}}{8}\right)\right.} \\
&\left.+\operatorname{tg} \mathrm{q}_{3}\left(\frac{\mathrm{x}_{\mathrm{r}}^{3}}{\ell_{\mathrm{r}}^{2}}+\frac{\mathrm{x}_{\mathrm{r}}^{2}}{2 \ell_{\mathrm{r}}}-\frac{\mathrm{x}_{\mathrm{r}}}{4}-\frac{\ell_{\mathrm{r}}}{8}\right)\right] \text { ou } \\
& \overline{\mathrm{v}}_{\mathrm{c}}=\left(1+\frac{\mathrm{q}_{1}}{\ell_{\mathrm{r}}}\right)\left[\operatorname{tg} \mathrm{q}_{2} \Psi_{2}+\operatorname{tg} \mathrm{q}_{3} \Psi_{3}\right]
\end{aligned}
$$

onde $\psi_{2}$ e $\psi_{3}$ são funções de forma, dadas por:

$$
\begin{aligned}
& \psi_{2}=\frac{x_{r}^{3}}{\ell_{r}^{2}}-\frac{x_{r}^{2}}{2 \ell_{r}}-\frac{x_{r}}{4}+\frac{\ell_{r}}{8} \\
& \psi_{3}=\frac{x_{r}^{3}}{\ell_{r}^{2}}+\frac{x_{r}^{2}}{2 \ell_{r}}-\frac{x_{r}}{4}-\frac{\ell_{r}}{8}
\end{aligned}
$$

e a $1^{\text {a }}$ derivada de $\bar{v}_{c}$ fica:

$$
\overline{\mathrm{v}}_{\mathrm{c}}^{\prime}=\left(1+\frac{\mathrm{q}_{1}}{\ell_{\mathrm{r}}}\right)\left[\operatorname{tg} \mathrm{q}_{2} \psi_{2}^{\prime}+\operatorname{tg} \mathrm{q}_{3} \psi_{3}^{\prime}\right]
$$

onde $\psi_{2}^{\prime}$ e $\psi_{3}^{\prime}$ são as derivadas de $\psi_{2}$ e $\psi_{3}$, dadas por:

$$
\begin{aligned}
& \psi_{2}^{\prime}=\frac{3 x_{r}^{2}}{\ell_{r}^{2}}-\frac{x_{r}}{\ell_{r}}-\frac{1}{4} \\
& \psi_{3}^{\prime}=\frac{3 x_{r}^{2}}{\ell_{r}^{2}}+\frac{x_{r}}{\ell_{r}}-\frac{1}{4}
\end{aligned}
$$

e a $2^{\underline{a}}$ derivada de $\bar{v}_{c}$ será: 


$$
{\overline{v_{c}}}^{\prime \prime}=\left(1+\frac{q_{1}}{\ell_{\mathrm{r}}}\right)\left[\operatorname{tg} \mathrm{q}_{2} \psi_{2}^{\prime \prime}+\operatorname{tg} \mathrm{q}_{3} \psi_{3}^{\prime \prime}\right]
$$

onde

$$
\begin{aligned}
& \psi_{2}^{\prime \prime}=\frac{6 \mathrm{x}_{\mathrm{r}}}{\ell_{\mathrm{r}}^{2}}-\frac{1}{\ell_{\mathrm{r}}} \\
& \psi_{3}^{\prime \prime}=\frac{6 \mathrm{x}_{\mathrm{r}}}{\ell_{\mathrm{r}}^{2}}+\frac{1}{\ell_{\mathrm{r}}}
\end{aligned}
$$

A expressão do campo de deformação $\varepsilon$, dada pela equação 3.149 , pode ser explicitada em termos dos deslocamentos nodais naturais $\left(\mathrm{q}_{1}, \mathrm{q}_{2}\right.$ e $\left.\mathrm{q}_{3}\right)$ e das funções de forma $\left(\psi_{2}\right.$ e $\left.\psi_{3}\right)$, resultando:

$$
\begin{aligned}
& \varepsilon=\left\{\left(1+\frac{\mathrm{q}_{1}}{\ell_{\mathrm{r}}}\right)^{2}\left[\left(1+\frac{\mathrm{q}_{1}}{\ell_{\mathrm{r}}}\right)\left(\operatorname{tg} \mathrm{q}_{2} \psi_{2}^{\prime}+\operatorname{tg} \mathrm{q}_{3} \psi_{3}^{\prime}\right)\right]^{2}\right\}^{1 / 2}-1 \\
& -\mathrm{y}_{\mathrm{r}}\left\{\frac{\left[\left(1+\frac{\mathrm{q}_{1}}{\ell_{\mathrm{r}}}\right)\left(1+\frac{\mathrm{q}_{1}}{\ell_{\mathrm{r}}}\right)\left(\operatorname{tg} \mathrm{q}_{2} \psi_{2}^{\prime \prime}+\operatorname{tg} \mathrm{q}_{3} \psi_{3}^{\prime \prime}\right)\right]^{2}}{\left(1+\frac{\mathrm{q}_{1}}{\ell_{\mathrm{r}}}\right)^{2}+\left[\left(1+\frac{\mathrm{q}_{1}}{\ell_{\mathrm{r}}}\right)\left(\operatorname{tg} \mathrm{q}_{2} \psi_{2}^{\prime}+\operatorname{tg} \mathrm{q}_{3} \psi_{3}^{\prime}\right)\right]^{2}}\right\}
\end{aligned}
$$

Esta expressão é geral e não é feita nenhuma limitação quanto à magnitude dos deslocamentos e das rotações. Como se observa, esta expressão é bastante complicada, por isso, serão feitas a seguir algumas aproximações de natureza geométrica. 


\subsection{Aproximações e Simplificações Adotadas}

Com o objetivo de facilitar a implementação, serão feitas algumas aproximações de natureza geométrica e adotadas algumas simplificações no comportamento do elemento.

\subsection{1- Aproximações de $2^{\mathfrak{a}}$ ordem}

Assumindo-se que o ângulo de rotação $\alpha$ é pequeno, da ordem de grandeza da raiz quadrada do valor absoluto da deformação longitudinal $\varepsilon$, o que pode ser obtido com elementos curtos, é razoável admitir, sem perda de generalidade da formulação, as seguintes aproximações de $2^{\underline{a}}$ ordem de suas funções trigonométricas:

$$
\operatorname{tg} \alpha=\alpha \quad ; \operatorname{sen} \alpha=\alpha \quad ; \cos \alpha=1-\frac{\alpha^{2}}{2} \quad ; \sec \alpha=1+\frac{\alpha^{2}}{2}
$$

Se as deformações máximas admitidas atingem o valor $\varepsilon=0.002$, o limite de rotação pequena pode ser fixado em torno de $0.045 \mathrm{rad}$ ou $2.56^{\circ}$.

PIMENTA \& SOLER (1989) salientam que a hipótese acima não impede a ocorrência de grandes curvaturas, desde que os elementos sejam suficientemente curtos. No caso dos pórticos de concreto armado, as curvaturas são sempre pequenas, sendo assim o ângulo $\alpha$ será pequeno, independentemente do comprimento dos elementos e a hipótese acima se justifica sempre.

Da equação 3.144 vem:

$$
\alpha=\operatorname{arctg} \frac{\overline{\mathrm{v}}_{\mathrm{c}}^{\prime}}{1+\overline{\mathrm{u}}_{\mathrm{c}}^{\prime}}
$$


substituindo-se $\overline{\mathrm{v}}_{\mathrm{c}}^{\prime}$ (eq. 3.183) e $\overline{\mathrm{u}}_{\mathrm{c}}^{\prime}$ (eq. 3.180), resulta:

$$
\begin{aligned}
& \alpha=\operatorname{arctg} \frac{\left(1+\frac{\mathrm{q}_{1}}{\ell_{\mathrm{r}}}\right)\left(\operatorname{tg} \mathrm{q}_{2} \psi_{2}^{\prime}+\operatorname{tg} \mathrm{q}_{3} \psi_{3}^{\prime}\right)}{\left(1+\frac{\mathrm{q}_{1}}{\ell_{\mathrm{r}}}\right)} \text { ou } \\
& \alpha=\operatorname{arctg}\left(\operatorname{tg} \mathrm{q}_{2} \psi_{2}^{\prime}+\operatorname{tg} \mathrm{q}_{3} \psi_{3}^{\prime}\right)
\end{aligned}
$$

Adotando-se as aproximações de $2^{\mathrm{a}}$ ordem $(\operatorname{tg} \alpha=\alpha)$, vem:

$$
\alpha=\mathrm{q}_{2} \psi_{2}^{\prime}+\mathrm{q}_{3} \psi_{3}^{\prime}
$$

derivando-se em relação a $\mathbf{x}_{\mathrm{r}}$, resulta:

$$
\alpha^{\prime}=\mathrm{q}_{2} \psi_{2}^{\prime \prime}+\mathrm{q}_{3} \psi_{3}^{\prime \prime}
$$

Levando-se (3.189) em (3.149), com o auxílio de (3.180) e considerando-se a aproximação $\sec \alpha=1+\frac{\alpha^{2}}{2}$, resulta:

$$
\begin{aligned}
& \varepsilon=\left(1+\frac{\mathrm{q}_{1}}{\ell_{\mathrm{r}}}\right)\left[1+\frac{\left(\mathrm{q}_{2} \psi_{2}^{\prime}+\mathrm{q}_{3} \psi_{3}^{\prime}\right)^{2}}{2}\right]-\mathrm{y}_{\mathrm{r}}\left(\mathrm{q}_{2} \psi_{2}^{\prime \prime}+\mathrm{q}_{3} \psi_{3}^{\prime \prime}\right)-1 \\
& \varepsilon=\frac{\mathrm{q}_{1}}{\ell_{\mathrm{r}}}+\frac{1}{2}\left(1+\frac{\mathrm{q}_{1}}{\ell_{\mathrm{r}}}\right)\left(\mathrm{q}_{2} \psi_{2}^{\prime}+\mathrm{q}_{3} \psi_{3}^{\prime}\right)^{2}-\mathrm{y}_{\mathrm{r}}\left(\mathrm{q}_{2} \psi_{2}^{\prime \prime}+\mathrm{q}_{3} \psi_{3}^{\prime \prime}\right)
\end{aligned}
$$


que é a expressão do campo de deformações quando se adota as aproximações de $2^{\mathrm{a}}$ ordem.

Para obtenção da matriz de rigidez tangente em coordenadas cartesianas será necessário efetuar as derivadas $\varepsilon_{, \alpha}$ e $\varepsilon_{, \alpha \beta}(\operatorname{com} \alpha=1,2,3$ e $\beta=1,2,3)$, assim:

$$
\left\{\begin{aligned}
& \varepsilon_{, 1}=\frac{\partial \varepsilon}{\partial \mathrm{q}_{1}}=\frac{1}{\ell_{\mathrm{r}}}+\frac{1}{2}\left(\frac{1}{\ell_{\mathrm{r}}}\right)\left(\mathrm{q}_{2} \psi_{2}^{\prime}+\mathrm{q}_{3} \psi_{3}^{\prime}\right)^{2}=\frac{1}{\ell_{\mathrm{r}}}\left(1+\frac{1}{2} \alpha^{2}\right) \\
& \varepsilon_{, 2}=\frac{\partial \varepsilon}{\partial \mathrm{q}_{2}}=\left(1+\frac{\mathrm{q}_{1}}{\ell_{\mathrm{r}}}\right)\left(\mathrm{q}_{2} \psi_{2}^{\prime}+\mathrm{q}_{3} \psi_{3}^{\prime}\right)\left(\psi_{2}^{\prime}\right)-\mathrm{y}_{\mathrm{r}} \psi_{2}^{\prime \prime} \\
&=\left(1+\frac{\mathrm{q}_{1}}{\ell_{\mathrm{r}}}\right) \alpha \psi_{2}^{\prime}-\mathrm{y}_{\mathrm{r}} \psi_{2}^{\prime \prime} \\
& \varepsilon_{, 3}=\frac{\partial \varepsilon}{\partial \mathrm{q}_{3}}=\left(1+\frac{\mathrm{q}_{1}}{\ell_{\mathrm{r}}}\right)\left(\mathrm{q}_{2} \psi_{2}^{\prime}+\mathrm{q}_{3} \psi_{3}^{\prime}\right)\left(\psi_{3}^{\prime}\right)-\mathrm{y}_{\mathrm{r}} \psi_{3}^{\prime \prime} \\
&=\left(1+\frac{\mathrm{q}_{1}}{\ell_{\mathrm{r}}}\right) \alpha \psi_{3}^{\prime}-\mathrm{y}_{\mathrm{r}} \psi_{3}^{\prime \prime} \\
& \varepsilon_{, 11}=\frac{\partial^{2} \varepsilon}{\partial \mathrm{q}_{1} \partial \mathrm{q}_{1}}=\frac{\partial \varepsilon_{, 1}}{\partial \mathrm{q}_{1}}=0
\end{aligned}\right.
$$




$$
\left\{\begin{array}{l}
\varepsilon_{, 12}=\frac{\partial^{2} \varepsilon}{\partial \mathrm{q}_{1} \partial \mathrm{q}_{2}}=\frac{\partial \varepsilon_{, 1}}{\partial \mathrm{q}_{2}}=\frac{1}{\ell_{\mathrm{r}}}\left(\mathrm{q}_{2} \psi_{2}^{\prime}+\mathrm{q}_{3} \psi_{3}^{\prime}\right)\left(\psi_{2}^{\prime}\right)=\frac{1}{\ell_{\mathrm{r}}} \alpha \psi_{2}^{\prime} \\
\varepsilon_{, 13}=\frac{\partial^{2} \varepsilon}{\partial \mathrm{q}_{1} \partial \mathrm{q}_{3}}=\frac{\partial \varepsilon_{, 1}}{\partial \mathrm{q}_{3}}=\frac{1}{\ell_{\mathrm{r}}}\left(\mathrm{q}_{2} \psi_{2}^{\prime}+\mathrm{q}_{3} \psi_{3}^{\prime}\right)\left(\Psi_{3}^{\prime}\right)=\frac{1}{\ell_{\mathrm{r}}} \alpha \psi_{3}^{\prime} \\
\varepsilon_{, 21}=\frac{\partial^{2} \varepsilon}{\partial \mathrm{q}_{2} \partial \mathrm{q}_{1}}=\frac{\partial \varepsilon_{, 2}}{\partial \mathrm{q}_{1}}=\frac{1}{\ell_{\mathrm{r}}} \alpha \psi_{2}^{\prime} \\
\text { então, } \varepsilon_{, 21}=\varepsilon_{, 12}(\text { simetria }) \\
\varepsilon_{, 22}=\frac{\partial^{2} \varepsilon}{\partial \mathrm{q}_{2} \partial \mathrm{q}_{2}}=\frac{\partial \varepsilon_{, 2}}{\partial \mathrm{q}_{2}}=\left(1+\frac{\mathrm{q}_{1}}{\ell_{\mathrm{r}}}\right) \Psi_{2}^{\prime} \Psi_{2}^{\prime} \\
\varepsilon_{, 23}=\frac{\partial^{2} \varepsilon}{\partial \mathrm{q}_{2} \partial \mathrm{q}_{3}}=\frac{\partial \varepsilon_{, 2}}{\partial \mathrm{q}_{3}}=\left(1+\frac{\mathrm{q}_{1}}{\ell_{\mathrm{r}}}\right) \Psi_{2}^{\prime} \Psi_{3}^{\prime} \\
\varepsilon_{, 31}=\frac{\partial^{2} \varepsilon}{\partial \mathrm{q}_{3} \partial \mathrm{q}_{1}}=\frac{\partial \varepsilon_{, 3}}{\partial \mathrm{q}_{1}}=\frac{1}{\ell_{\mathrm{r}}} \alpha \psi_{3}^{\prime} \\
\text { então, } \varepsilon_{, 31}=\varepsilon_{, 13}(\text { simetria }) \\
\varepsilon_{, 32}=\frac{\partial^{2} \varepsilon}{\partial \mathrm{q}_{3} \partial \mathrm{q}_{2}}=\frac{\partial \varepsilon_{, 3}}{\partial \mathrm{q}_{2}}=\left(1+\frac{\mathrm{q}_{1}}{\ell_{\mathrm{r}}}\right) \Psi_{2}^{\prime} \Psi_{3}^{\prime} \\
\text { então, } \varepsilon_{, 32}=\varepsilon_{, 23}(\text { simetria }) \\
\varepsilon_{, 33}=\frac{\partial^{2} \varepsilon}{\partial \mathrm{q}_{3} \partial \mathrm{q}_{3}}=\frac{\partial \varepsilon_{, 3}}{\partial \mathrm{q}_{3}}=\left(1+\frac{\mathrm{q}_{1}}{\ell_{\mathrm{r}}}\right) \Psi_{3}^{\prime} \Psi_{3}^{\prime}
\end{array}\right.
$$

lembrando-se que o estiramento $\lambda=\ell_{\mathrm{c}} / \ell_{\mathrm{r}}$ e $\mathrm{q}_{1}=\ell_{\mathrm{c}}-\ell_{\mathrm{r}}$, então:

$$
\begin{aligned}
& \lambda=\frac{\ell_{\mathrm{c}}}{\ell_{\mathrm{r}}} \Rightarrow \lambda-1=\frac{\ell_{\mathrm{c}}}{\ell_{\mathrm{r}}}-\frac{\ell_{\mathrm{r}}}{\ell_{\mathrm{r}}} \Rightarrow \lambda-1=\frac{\ell_{\mathrm{c}}-\ell_{\mathrm{r}}}{\ell_{\mathrm{r}}} \Rightarrow \lambda-1=\frac{\mathrm{q}_{1}}{\ell_{\mathrm{r}}} \\
& \lambda=1+\frac{\mathrm{q}_{1}}{\ell_{\mathrm{r}}}
\end{aligned}
$$

substituindo-se (3.193) em (3.191) e (3.192), vem:

$$
\left\{\begin{array}{l}
\varepsilon_{, 1}=\frac{1}{\ell_{\mathrm{r}}}\left(1+\frac{1}{2} \alpha^{2}\right) \\
\varepsilon_{, 2}=\lambda \alpha \psi_{2}^{\prime}-\mathrm{y}_{\mathrm{r}} \psi_{2}^{\prime \prime} \\
\varepsilon_{, 3}=\lambda \alpha \psi_{3}^{\prime}-\mathrm{y}_{\mathrm{r}} \psi_{3}^{\prime \prime} \\
\varepsilon_{, 11}=0
\end{array}\right.
$$




$$
\left\{\begin{array}{l}
\varepsilon_{, 12}=\varepsilon_{, 21}=1 / \ell_{\mathrm{r}} \alpha \psi_{2}^{\prime} \\
\varepsilon_{.13}=\varepsilon_{, 31}=1 / \ell_{\mathrm{r}} \alpha \psi_{3}^{\prime} \\
\varepsilon_{, 22}=\lambda \psi_{2}^{\prime} \psi_{2}^{\prime} \\
\varepsilon_{, 23}=\varepsilon_{, 32}=\lambda \psi_{2}^{\prime} \psi_{3}^{\prime} \\
\varepsilon_{., 33}=\lambda \psi_{3}^{\prime} \psi_{3}^{\prime}
\end{array}\right.
$$

Os esforços nodais naturais (eq. 3.161), após as simplificações de $2^{\underline{a}}$ ordem, serão:

$$
Q_{1}=\int_{-t_{r} / 2}^{t_{r} / 2} \int_{A_{r}} \sigma \varepsilon_{, 1} \mathrm{dA}_{\mathrm{r}} \mathrm{d} x_{\mathrm{r}} \quad \text { ou }
$$

com auxílio de (3.194)

$$
\mathrm{Q}_{1}=\int_{-\ell_{\mathrm{r}} / 2}^{\ell_{\mathrm{r}} / 2} \frac{1}{\ell_{\mathrm{r}}}\left(1+\frac{\alpha^{2}}{2}\right) \int_{\mathrm{A}_{\mathrm{r}}} \sigma \mathrm{dA}_{\mathrm{r}} \mathrm{dx} \mathrm{x}_{\mathrm{r}}
$$

resultando:

$$
\begin{aligned}
& Q_{1}=\int_{-\ell_{r} / 2}^{\ell_{r} / 2} N \frac{1}{\ell_{r}}\left(1+\frac{\alpha^{2}}{2}\right) d x_{r} \\
& Q_{2}=\int_{-\ell_{r} / 2}^{\ell_{r} / 2} \int_{A_{r}} \sigma \varepsilon_{, 2} d A_{r} d x_{r} \text { ou }
\end{aligned}
$$

com auxílio de (3.194)

$$
\begin{aligned}
& \mathrm{Q}_{2}=\int_{-\ell_{\mathrm{r}} / 2}^{\ell_{\mathrm{r}} / 2} \int_{\mathrm{A}_{\mathrm{r}}}\left(\lambda \alpha \psi_{2}^{\prime}-\mathrm{y}_{\mathrm{r}} \psi_{2}^{\prime \prime}\right) \sigma \mathrm{dA}_{\mathrm{r}} \mathrm{dx}_{\mathrm{r}} \quad \text { ou } \\
& \mathrm{Q}_{2}=\int_{-\ell_{\mathrm{r}} / 2}^{\ell_{\mathrm{r}} / 2} \lambda \alpha \psi_{2}^{\prime} \int_{\mathrm{A}_{\mathrm{r}}} \sigma \mathrm{dA}_{\mathrm{r}} \mathrm{d} \mathrm{x}_{\mathrm{r}}-\int_{-\ell_{\mathrm{r}} / 2}^{\ell_{\mathrm{r}} / 2} \psi_{2}^{\prime \prime} \int_{\mathrm{A}_{\mathrm{r}}} \sigma \mathrm{y}_{\mathrm{r}} \mathrm{dA} \mathrm{A}_{\mathrm{r}} \mathrm{dx}_{\mathrm{r}} \quad \text { ou }
\end{aligned}
$$




$$
\begin{aligned}
& \mathrm{Q}_{2}=\int_{-\ell_{\mathrm{r}} / 2}^{\ell_{\mathrm{r}} / 2}\left(\mathrm{~N} \lambda \alpha \psi_{2}^{\prime}+\mathrm{M} \psi_{2}^{\prime \prime}\right) \mathrm{d} \mathrm{x}_{\mathrm{r}} \\
& \mathrm{Q}_{3}=\int_{-\ell_{\mathrm{r}} / 2}^{\ell_{\mathrm{r}} / 2} \int_{\mathrm{A}_{\mathrm{r}}} \sigma \varepsilon_{, 3} \mathrm{dA} \mathrm{A}_{\mathrm{r}} \mathrm{dx}_{\mathrm{r}} \text { ou }
\end{aligned}
$$

com auxílio de (3.194)

$$
\begin{aligned}
& \mathrm{Q}_{3}=\int_{-\ell_{\mathrm{r}} / 2}^{\ell_{\mathrm{r}} / 2} \int_{\mathrm{A}_{\mathrm{r}}}\left(\lambda \alpha \psi_{3}^{\prime}-\mathrm{y}_{\mathrm{r}} \psi_{3}^{\prime \prime}\right) \sigma \mathrm{dA}_{\mathrm{r}} \mathrm{dx}_{\mathrm{r}} \quad \text { ou } \\
& \mathrm{Q}_{3}=\int_{-\ell_{\mathrm{r}} / 2}^{\ell_{\mathrm{r}} / 2} \lambda \alpha \psi_{3}^{\prime} \int_{\mathrm{A}_{\mathrm{r}}} \sigma \mathrm{d} \mathrm{A}_{\mathrm{r}} \mathrm{dx} \mathrm{x}_{\mathrm{r}}-\int_{-\ell_{\mathrm{r}} / 2}^{\ell_{\mathrm{r}} / 2} \psi_{3}^{\prime \prime} \int_{\mathrm{A}_{\mathrm{r}}} \sigma \mathrm{y}_{\mathrm{r}} \mathrm{dA} \mathrm{A}_{\mathrm{r}} \mathrm{dx} \mathrm{r}_{\mathrm{r}} \text { ou } \\
& \mathrm{Q}_{3}=\int_{-\ell_{\mathrm{r}} / 2}^{\ell_{\mathrm{r}} / 2}\left(\mathrm{~N} \lambda \alpha \psi_{3}^{\prime}+\mathrm{M} \psi_{3}^{\prime \prime}\right) \mathrm{d} \mathrm{x}_{\mathrm{r}}
\end{aligned}
$$

onde:

$\mathrm{N}$ é a força normal e M o momento fletor nas seções transversais do elemento, definidos de forma usual por:

$$
\begin{aligned}
& N=\int_{A_{r}} \sigma d A_{r}=\int_{-h / 2}^{h / 2} \sigma b d y_{r} \\
& M=-\int_{A_{r}} \sigma y_{r} d A_{r}=-\int_{-h / 2}^{h / 2} \sigma b y_{r} d y_{r}
\end{aligned}
$$

onde b e h são, respectivamente, a largura e a altura da seção transversal.

Os elementos da matriz $\mathbf{H}$, necessária para a obtenção de $\mathbf{k}_{\mathrm{G}}$ (eq. 3.176), após as simplificações de $2^{2}$ ordem, podem ser obtidos com auxílio de (3.168) e (3.195), ou seja: 


$$
\begin{aligned}
& \mathrm{H}_{11}=\int_{-\ell_{\mathrm{r}} / 2}^{\ell_{\mathrm{r}} / 2} \int_{\mathrm{A}_{\mathrm{r}}} \sigma \varepsilon_{, 11} \mathrm{dA}_{\mathrm{r}} \mathrm{dx}_{\mathrm{r}} \Rightarrow \mathrm{H}_{11}=0 \\
& \mathrm{H}_{12}=\int_{-\ell_{\mathrm{r}} / 2}^{\ell_{\mathrm{r}} / 2} \int_{\mathrm{A}_{\mathrm{r}}} \sigma \varepsilon_{, 12} \mathrm{dA}_{\mathrm{r}} \mathrm{dx}_{\mathrm{r}}=\int_{-\ell_{\mathrm{r}} / 2}^{\ell_{\mathrm{r}} / 2} \int_{\mathrm{A}_{\mathrm{r}}} \sigma \frac{1}{\ell_{\mathrm{r}}} \alpha \psi_{2}^{\prime} \mathrm{dA}_{\mathrm{r}} \mathrm{dx} \mathrm{x}_{\mathrm{r}} \\
& \mathrm{H}_{12}=\int_{-\ell_{\mathrm{r}} / 2}^{\ell_{\mathrm{r}} / 2} \frac{1}{\ell_{\mathrm{r}}} \alpha \psi_{2}^{\prime} \int_{\mathrm{A}_{\mathrm{r}}} \sigma \mathrm{dA}_{\mathrm{r}} \mathrm{dx} \mathrm{x}_{\mathrm{r}} \quad \text { ou } \\
& \mathrm{H}_{12}=\int_{-\ell_{\mathrm{r}} / 2}^{\ell_{\mathrm{r}} / 2} \mathrm{~N} \frac{1}{\ell_{\mathrm{r}}} \alpha \psi_{2}^{\prime} \mathrm{dx}
\end{aligned}
$$

Analogamente, obtém-se:

$$
\begin{aligned}
& \mathrm{H}_{13}=\mathrm{H}_{31}=\int_{-\ell_{\mathrm{r}} / 2}^{\ell_{\mathrm{r}} / 2} \mathrm{~N} \frac{1}{\ell_{\mathrm{r}}} \alpha \psi_{3}^{\prime} \mathrm{dx} \mathrm{x}_{\mathrm{r}} \\
& \mathrm{H}_{22}=\int_{-\ell_{\mathrm{r}} / 2}^{\ell_{\mathrm{r}} / 2} \mathrm{~N} \lambda \psi_{2}^{\prime} \psi_{2}^{\prime} \mathrm{d} \mathrm{x}_{\mathrm{r}} \\
& \mathrm{H}_{23}=\mathrm{H}_{32}=\int_{-\ell_{\mathrm{r}} / 2}^{\ell_{\mathrm{r}} / 2} \mathrm{~N} \lambda \psi_{2}^{\prime} \psi_{3}^{\prime} \mathrm{dx} \mathrm{r}_{\mathrm{r}} \\
& \mathrm{H}_{33}=\int_{-\ell_{\mathrm{r}} / 2}^{\ell_{\mathrm{r}} / 2} \mathrm{~N} \lambda \psi_{3}^{\prime} \psi_{3}^{\prime} \mathrm{dx} \mathrm{x}_{\mathrm{r}}
\end{aligned}
$$

Os elementos da matriz $\mathbf{D}$, necessária para a obtenção de $\mathbf{k}_{\mathbf{M}}$, após as simplificações de $2^{\underline{a}}$ ordem, ficam com auxílio de (3.169) e (3.194):

$$
D_{11}=\int_{-\ell_{r} / 2}^{\ell_{r} / 2} \int_{A_{r}} D \varepsilon_{, 1} \varepsilon_{, 1} \mathrm{dA}_{r} d_{x_{r}}=\int_{-\ell_{r} / 2}^{\ell_{r} / 2} \int_{A_{r}} D\left[\frac{1}{\ell_{r}}\left(1+\frac{\alpha^{2}}{2}\right)\right]^{2} \mathrm{dA}_{r} d_{x_{r}} \text { ou }
$$




$$
\begin{aligned}
& D_{11}=\int_{-\ell_{r} / 2}^{\ell_{r} / 2} \frac{1}{\ell_{r}^{2}}\left(1+\frac{\alpha^{2}}{2}\right)^{2} \int_{A_{r}} D_{d A_{r}} d x_{r} \quad 0 u \\
& D_{11}=\int_{-\ell_{r} / 2}^{\ell_{r} / 2} C_{1} \frac{1}{\ell_{r}^{2}}\left(1+\frac{\alpha^{2}}{2}\right)^{2} d x_{r} \\
& D_{12}=\int_{-\ell_{\mathrm{r}} / 2}^{\ell_{\mathrm{r}} / 2} \int_{\mathrm{A}_{\mathrm{r}}} \mathrm{D} \varepsilon_{, 1} \varepsilon_{, 2} \mathrm{dA} \mathrm{A}_{\mathrm{r}} \mathrm{dx} \\
& =\int_{-\ell_{\mathrm{r}} / 2}^{\ell_{\mathrm{r}} / 2} \int_{\mathrm{A}_{\mathrm{r}}} \mathrm{D} \frac{1}{\ell_{\mathrm{r}}}\left(1+\frac{\alpha^{2}}{2}\right)\left(\lambda \alpha \psi_{2}^{\prime}-\mathrm{y}_{\mathrm{r}} \psi_{2}^{\prime \prime}\right) \mathrm{dA}_{\mathrm{r}} \mathrm{dx}_{\mathrm{r}} \\
& D_{12}=\int_{-\ell_{r} / 2}^{\ell_{\mathrm{r}} / 2} \frac{1}{\ell_{\mathrm{r}}}\left(1+\frac{\alpha^{2}}{2}\right)\left(\lambda \alpha \psi_{2}^{\prime}\right) \int_{\mathrm{A}_{\mathrm{r}}} \mathrm{D} \mathrm{dA}_{\mathrm{r}} \mathrm{dx}_{\mathrm{r}} \\
& -\int_{-\ell_{\mathrm{r}} / 2}^{\ell_{\mathrm{r}} / 2} \frac{1}{\ell_{\mathrm{r}}}\left(1+\frac{\alpha^{2}}{2}\right) \psi_{2}^{\prime \prime} \int_{\mathrm{A}_{\mathrm{r}}} D \mathrm{y}_{\mathrm{r}} \mathrm{dA} \mathrm{A}_{\mathrm{r}} \mathrm{dx} \\
& \mathrm{D}_{12}=\mathrm{D}_{21}=\int_{-\ell_{\mathrm{r}} / 2}^{\ell_{\mathrm{r}} / 2}\left(1+\frac{\alpha^{2}}{2}\right)\left(\frac{\mathrm{C}_{1} \lambda \alpha \psi_{2}^{\prime}}{\ell_{\mathrm{r}}}-\frac{\mathrm{C}_{2} \psi_{2}^{\prime \prime}}{\ell_{\mathrm{r}}}\right) \mathrm{d} \mathrm{x}_{\mathrm{r}}
\end{aligned}
$$

ou

Analogamente, obtém-se:

$$
\begin{gathered}
\mathrm{D}_{13}=\mathrm{D}_{31}=\int_{-\ell_{\mathrm{r}} / 2}^{\ell_{\mathrm{r}} / 2}\left(1+\frac{\alpha^{2}}{2}\right)\left(\frac{\mathrm{C}_{1} \lambda \alpha \psi_{3}^{\prime}}{\ell_{\mathrm{r}}}-\frac{\mathrm{C}_{2} \psi_{3}^{\prime \prime}}{\ell_{\mathrm{r}}}\right) \mathrm{dx}_{\mathrm{r}} \\
\mathrm{D}_{22}=\int_{-\ell_{\mathrm{r}} / 2}^{\ell_{\mathrm{r}} / 2}\left(\mathrm{C}_{1} \lambda^{2} \alpha^{2} \psi_{2}^{\prime} \psi_{2}^{\prime}-2 \mathrm{C}_{2} \lambda \alpha \psi_{2}^{\prime} \psi_{2}^{\prime \prime}+\mathrm{C}_{3} \psi_{2}^{\prime \prime} \psi_{2}^{\prime \prime}\right) \mathrm{d} \mathrm{x}_{\mathrm{r}}(3.210) \\
\mathrm{D}_{23}=\mathrm{D}_{32}=\int_{-\ell_{\mathrm{r}} / 2}^{\ell_{\mathrm{r}} / 2}\left[\mathrm{C}_{1} \lambda^{2} \alpha^{2} \psi_{2}^{\prime} \psi_{3}^{\prime}-\mathrm{C}_{2} \lambda \alpha\left(\psi_{2}^{\prime} \psi_{3}^{\prime \prime}+\psi_{3}^{\prime} \psi_{2}^{\prime \prime}\right)\right. \\
\left.+\mathrm{C}_{3} \psi_{2}^{\prime \prime} \psi_{3}^{\prime \prime}\right] \mathrm{d} \mathrm{x}_{\mathrm{r}}
\end{gathered}
$$




$$
\mathrm{D}_{33}=\int_{-\ell_{\mathrm{r}} / 2}^{\ell_{\mathrm{r}} / 2}\left(\mathrm{C}_{1} \lambda^{2} \alpha^{2} \psi_{3}^{\prime} \psi_{3}^{\prime}-2 \mathrm{C}_{2} \lambda \alpha \psi_{3}^{\prime} \psi_{3}^{\prime \prime}+\mathrm{C}_{3} \psi_{3}^{\prime \prime} \psi_{3}^{\prime \prime}\right) \mathrm{dx_{ \textrm {r } }}
$$

onde:

$$
\begin{aligned}
& C_{1}=\int_{A_{r}} D d A_{r}=\int_{-h / 2}^{h / 2} D b d y_{r} \\
& C_{2}=\int_{A_{r}} D y_{r} d A_{r}=\int_{-h / 2}^{h / 2} D b y_{r} d y_{r} \\
& C_{3}=\int_{A_{r}} D y_{r}^{2} d A_{r}=\int_{-h / 2}^{h / 2} D b y_{r}^{2} d y_{r}
\end{aligned}
$$

Em regime elástico linear ter-se-ia:

$$
\mathrm{C}_{1}=\mathrm{EA}_{\mathrm{r}} \quad ; \mathrm{C}_{2}=0 \quad ; \mathrm{C}_{3}=\mathrm{EI}
$$

Em regime elástico não-linear e regime elasto-plástico, os valores de $\mathrm{C}_{1}, \mathrm{C}_{2}$ e $\mathrm{C}_{3}$ serão constantes na seção, obtidos pela integração das equações (3.213) e (3.215), mas podem variar ao longo de $\mathrm{x}_{\mathrm{r}}$, pois a rigidez tangente "D" pode variar com $\mathbf{x}_{\mathrm{r}}$.

As integrais para obtenção de $\mathrm{Q}_{\alpha}$ (equações 3.196, 3.197 e 3.198), $\mathrm{H}_{\alpha \beta}$ (equações 3.201 a 3.206) e $D_{\alpha \beta}$ (equações 3.207 a 3.212) podem ser computadas numericamente através do método de Gauss.

As integrais para obtenção de $\mathrm{N}$ (eq. 3.199), $\mathrm{M}$ (eq. 3.200), $\mathrm{C}_{1}$ (eq. 3.213), $C_{2}$ (eq. 3.214) e $C_{3}$ (eq. 3.215) podem ser calculadas nos pontos de integração de Gauss pelo método dos retângulos, com um grande número de pontos de integração, para poder captar a variação significativa dos valores de $\sigma$ e $D$, nos diversos pontos da seção transversal e ao longo de $x_{r}$, principalmente em problemas elasto-plásticos. 


\subsection{2 - Elemento Prismático em Regime Elástico Linear}

Para um elemento prismático em regime elástico linear, tem-se que a rigidez tangente $\left(\mathrm{D}=\frac{\mathrm{d} \sigma}{\mathrm{d} \varepsilon}\right)$ é constante e igual ao módulo de elasticidade longitudinal $\mathrm{E}$.

Da expressão do campo de deformação $\varepsilon$ dado por (3.190), tem-se que a deformação da fibra média $\bar{\varepsilon}$ é obtida fazendo $\mathrm{y}_{\mathrm{r}}=0$, isto é,

$$
\bar{\varepsilon}=\frac{\mathrm{q}_{1}}{\ell_{\mathrm{r}}}+\frac{1}{2}\left(1+\frac{\mathrm{q}_{1}}{\ell_{\mathrm{r}}}\right)\left(\mathrm{q}_{2} \psi_{2}^{\prime}+\mathrm{q}_{3} \psi_{3}^{\prime}\right)^{2}
$$

variável ao longo do elemento, uma vez que $\psi_{2}^{\prime}$ e $\psi_{3}^{\prime}$ são função de $\mathbf{x}_{\mathrm{r}}$ (eq. $3.183 a$ e $3.183 b)$.

Com o objetivo de se resolver analiticamente as integrais para obtenção de $Q_{\alpha}, H_{\alpha \beta}$ e $D_{\alpha \beta}$, substituir-se-á o valor variável $\bar{\varepsilon}$ pelo seu valor médio e constante, dado por:

$$
\begin{aligned}
& \bar{\varepsilon}_{\mathrm{m}}=\frac{1}{\ell_{\mathrm{r}}} \int_{-\ell_{\mathrm{r}} / 2}^{\ell_{\mathrm{r}} / 2} \bar{\varepsilon} \mathrm{dx_{ \textrm {r } }} \text { ou } \\
& \bar{\varepsilon}_{\mathrm{m}}=\frac{1}{\ell_{\mathrm{r}}} \int_{-\ell_{\mathrm{r}} / 2}^{\ell_{\mathrm{r}} / 2}\left[\frac{\mathrm{q}_{1}}{\ell_{\mathrm{r}}}+\frac{1}{2}\left(1+\frac{\mathrm{q}_{1}}{\ell_{\mathrm{r}}}\right)\left(\mathrm{q}_{2} \psi_{2}^{\prime}+\mathrm{q}_{3} \psi_{3}^{\prime}\right)^{2}\right] \mathrm{dx_{ \textrm {r } }}
\end{aligned}
$$

substituindo-se $\psi_{2}^{\prime}$ e $\psi_{3}^{\prime}$ pelas expressões dadas por (3.183a e $3.183 \mathrm{~b}$ ), e resolvendo-se analiticamente a integral tem-se:

$$
\bar{\varepsilon}_{\mathrm{m}}=\frac{\mathrm{q}_{1}}{\ell_{\mathrm{r}}}+\left(1+\frac{\mathrm{q}_{1}}{\ell_{\mathrm{r}}}\right)\left(\frac{\mathrm{q}_{2}^{2}}{15}+\frac{\mathrm{q}_{3}^{2}}{15}-\frac{\mathrm{q}_{2} \mathrm{q}_{3}}{30}\right)
$$


e o campo de deformação $\left(\varepsilon=\bar{\varepsilon}-y_{\mathrm{r}} \alpha^{\prime}\right)$, com esta simplificação, fica:

$$
\varepsilon=\frac{\mathrm{q}_{1}}{\ell_{\mathrm{r}}}+\left(1+\frac{\mathrm{q}_{1}}{\ell_{\mathrm{r}}}\right)\left(\frac{\mathrm{q}_{2}^{2}}{15}+\frac{\mathrm{q}_{3}^{2}}{15}-\frac{\mathrm{q}_{2} \mathrm{q}_{3}}{30}\right)-\mathrm{y}_{\mathrm{r}}\left(\mathrm{q}_{2} \psi_{2}^{\prime \prime}+\mathrm{q}_{3} \psi_{3}^{\prime \prime}\right)
$$

Os esforços solicitantes serão:

$$
\begin{aligned}
& N=\int_{A_{r}} \sigma d A_{r}=\int_{A_{r}} E \varepsilon d A_{r} \\
& N=\int_{A_{r}} E\left(\bar{\varepsilon}_{\mathrm{m}}-y_{r} \alpha^{\prime}\right) d A_{r}=\int_{A_{r}} E \bar{\varepsilon}_{\mathrm{m}} d A_{r}-\int_{A_{r}} E \alpha^{\prime} y_{r} d A_{r} \\
& N=E A_{r} \bar{\varepsilon}_{m} \\
& M=-\int_{A_{r}} \sigma y_{r} d A_{r}=-\int_{A_{r}} E \varepsilon y_{r} d A_{r}=-\int_{A_{r}} E\left(\bar{\varepsilon}-y_{r} \alpha^{\prime}\right) y_{r} d A_{r} \\
& M=-\int_{A_{r}} E \bar{\varepsilon} y_{r} d A_{r}+\int_{A_{r}} E \alpha^{\prime} y_{r}^{2} d A_{r} \\
& M=E I \alpha^{\prime}=E I\left(q_{2} \psi_{2}^{\prime \prime}+q_{3} \psi_{3}^{\prime \prime}\right) \quad \text { ou } \\
& M=E I\left[q_{2}\left(\frac{6 x_{r}}{\ell_{r}^{2}}-\frac{1}{\ell_{r}}\right)+q_{3}\left(\frac{6 x_{r}}{\ell_{r}^{2}}+\frac{1}{\ell_{r}}\right)\right] \\
& x_{r}=+\frac{\ell}{2} \Rightarrow M_{B}=+\frac{E I}{\ell_{r}}\left(2 q_{2}+4 q_{3}\right) \\
& x_{r}=-\frac{\ell_{r}}{2} \Rightarrow M_{A}=-\frac{E I}{\ell}\left(4 q_{2}+2 q_{3}\right)
\end{aligned}
$$


As derivadas $\varepsilon_{, \alpha}$ e $\varepsilon_{, \alpha \beta}$ ficam:

$$
\begin{aligned}
& \varepsilon_{, 1}=\frac{1}{\ell_{\mathrm{r}}}+\frac{1}{\ell_{\mathrm{r}}}\left(\frac{\mathrm{q}_{2}^{2}}{15}+\frac{\mathrm{q}_{3}^{2}}{15}-\frac{\mathrm{q}_{2} \mathrm{q}_{3}}{30}\right) \\
& \varepsilon_{, 2}=\left(1+\frac{\mathrm{q}_{1}}{\ell_{\mathrm{r}}}\right)\left(\frac{2 \mathrm{q}_{2}}{15}-\frac{\mathrm{q}_{3}}{30}\right)-\mathrm{y}_{\mathrm{r}} \psi_{2}^{\prime \prime} \\
& \varepsilon_{, 3}=\left(1+\frac{\mathrm{q}_{1}}{\ell_{\mathrm{r}}}\right)\left(\frac{2 \mathrm{q}_{3}}{15}-\frac{\mathrm{q}_{2}}{30}\right)-\mathrm{y}_{\mathrm{r}} \psi_{3}^{\prime \prime} \\
& \varepsilon_{, 11}=0 \\
& \varepsilon_{, 12}=\frac{1}{\ell_{\mathrm{r}}}\left(\frac{2 \mathrm{q}_{2}}{15}-\frac{\mathrm{q}_{3}}{30}\right) \\
& \varepsilon_{, 13}=\frac{1}{\ell_{\mathrm{r}}}\left(\frac{2 \mathrm{q}_{3}}{15}-\frac{\mathrm{q}_{2}}{30}\right) \\
& \varepsilon_{, .23}=\left(1+\frac{\mathrm{q}_{1}}{\ell_{\mathrm{r}}}\right)\left(-\frac{1}{30}\right)=-\frac{\lambda}{30} \\
& \varepsilon_{, 22}=\left(1+\frac{\mathrm{q}_{1}}{\ell_{\mathrm{r}}}\right) \frac{2}{15}=\frac{2 \lambda}{15} \\
& \varepsilon_{, 12}
\end{aligned}
$$


128

$$
\begin{aligned}
& \varepsilon_{, 32}=\varepsilon_{, 23} \\
& \varepsilon_{, 33}=\left(1+\frac{\mathrm{q}_{1}}{\ell_{\mathrm{r}}}\right) \frac{2}{15}=\frac{2 \lambda}{15}
\end{aligned}
$$

Os esforços nodais naturais (eq. 3.161) são dados por:

$$
\begin{aligned}
& Q_{1}=\int_{-\ell_{r} / 2}^{\ell_{r} / 2} \int_{A_{r}} \sigma \varepsilon_{, 1} d A_{r} d_{x_{r}} \quad o u \\
& \mathrm{Q}_{1}=\int_{-\ell_{\mathrm{r}} / 2}^{\ell_{\mathrm{r}} / 2} \int_{\mathrm{A}_{\mathrm{r}}} \sigma\left[\frac{1}{\ell_{\mathrm{r}}}+\frac{1}{\ell_{\mathrm{r}}}\left(\frac{\mathrm{q}_{2}^{2}}{15}+\frac{\mathrm{q}_{3}^{2}}{15}-\frac{\mathrm{q}_{2} \mathrm{q}_{3}}{30}\right)\right] \mathrm{dA}_{\mathrm{r}} \mathrm{dx}_{\mathrm{r}} \quad \text { ou } \\
& \mathrm{Q}_{1}=\mathrm{N}\left[\frac{1}{\ell_{\mathrm{r}}}+\frac{1}{\ell_{\mathrm{r}}}\left(\frac{\mathrm{q}_{2}^{2}}{15}+\frac{\mathrm{q}_{3}^{2}}{15}-\frac{\mathrm{q}_{2} \mathrm{q}_{3}}{30}\right)\right] \int_{-\ell_{\mathrm{r}} / 2}^{\ell_{\mathrm{r}} / 2} \mathrm{~d} \mathrm{x}_{\mathrm{r}} \quad \text { ou } \\
& \mathrm{Q}_{1}=\mathrm{N}\left[\frac{1}{\ell_{\mathrm{r}}}+\frac{1}{\ell_{\mathrm{r}}}\left(\frac{\mathrm{q}_{2}^{2}}{15}+\frac{\mathrm{q}_{3}^{2}}{15}-\frac{\mathrm{q}_{2} \mathrm{q}_{3}}{30}\right)\right] \ell_{\mathrm{r}} \quad \text { ou } \\
& \mathrm{Q}_{1}=\mathrm{N}\left(1+\frac{\mathrm{q}_{2}^{2}}{15}+\frac{\mathrm{q}_{3}^{2}}{15}-\frac{\mathrm{q}_{2} \mathrm{q}_{3}}{30}\right) \\
& \mathrm{Q}_{2}=\int_{-\ell_{\mathrm{r}} / 2}^{\ell_{\mathrm{r}} / 2} \int_{\mathrm{A}_{\mathrm{r}}} \sigma \varepsilon_{, 2} \mathrm{dA}_{\mathrm{r}} \mathrm{dx}_{\mathrm{r}} \quad \text { ou } \\
& \mathrm{Q}_{2}=\int_{-\ell_{\mathrm{r}} / 2}^{\ell_{\mathrm{r}} / 2} \int_{\mathrm{A}_{\mathrm{r}}} \sigma\left[\left(1+\frac{\mathrm{q}_{1}}{\ell_{\mathrm{r}}}\right)\left(\frac{2 \mathrm{q}_{2}}{15}-\frac{\mathrm{q}_{3}}{30}\right)-\mathrm{y}_{\mathrm{r}}\left(\frac{6 \mathrm{x}_{\mathrm{r}}}{\ell_{\mathrm{r}}^{2}}-\frac{1}{\ell_{\mathrm{r}}}\right)\right] \mathrm{dA}_{\mathrm{r}} \mathrm{d} \mathrm{x}_{\mathrm{r}} \quad \text { ou } \\
& \mathrm{Q}_{2}=\int_{-\ell_{\mathrm{r}} / 2}^{\ell_{\mathrm{r}} / 2}\left[\left(1+\frac{\mathrm{q}_{1}}{\ell_{\mathrm{r}}}\right)\left(\frac{2 \mathrm{q}_{2}}{15}-\frac{\mathrm{q}_{3}}{30}\right) \mathrm{N}+\left(\frac{6 \mathrm{x}_{\mathrm{r}}}{\ell_{\mathrm{r}}^{2}}-\frac{1}{\ell_{\mathrm{r}}}\right) \mathrm{M}\right] \mathrm{dx_{ \textrm {r } }} \text { ou }
\end{aligned}
$$




$$
\begin{aligned}
& \mathrm{Q}_{2}=\mathrm{N}\left[\left(1+\frac{\mathrm{q}_{1}}{\ell_{\mathrm{r}}}\right)\left(\frac{2 \mathrm{q}_{2}}{15}-\frac{\mathrm{q}_{3}}{30}\right)\right] \int_{-\ell_{\mathrm{r}} / 2}^{\ell_{\mathrm{r}} / 2} \mathrm{dx} \\
& +\int_{-\ell_{\mathrm{r}} / 2}^{\ell_{\mathrm{r}} / 2}\left(\frac{6 \mathrm{x}_{\mathrm{r}}}{\ell_{\mathrm{r}}^{2}}-\frac{1}{\ell_{\mathrm{r}}}\right) \mathrm{EI}\left[\mathrm{q}_{2}\left(\frac{6 \mathrm{x}_{\mathrm{r}}}{\ell_{\mathrm{r}}^{2}}-\frac{1}{\ell_{\mathrm{r}}}\right)+\mathrm{q}_{3}\left(\frac{6 \mathrm{x}_{\mathrm{r}}}{\ell_{\mathrm{r}}^{2}}+\frac{1}{\ell_{\mathrm{r}}}\right)\right] \mathrm{dx}_{\mathrm{r}} \\
& \mathrm{Q}_{2}=\mathrm{N} \ell_{\mathrm{r}}\left(1+\frac{\mathrm{q}_{1}}{\ell_{\mathrm{r}}}\right)\left(\frac{2 \mathrm{q}_{2}}{15}-\frac{\mathrm{q}_{3}}{30}\right)+\frac{4 \mathrm{EI}}{\ell_{\mathrm{r}}} \mathrm{q}_{2}+\frac{2 \mathrm{EI}}{\ell_{\mathrm{r}}} \mathrm{q}_{3} \quad \text { ou } \\
& \mathrm{Q}_{2}=\mathrm{N} \ell_{\mathrm{c}}\left(\frac{2 \mathrm{q}_{2}}{15}-\frac{\mathrm{q}_{3}}{30}\right)-\mathrm{M}_{\mathrm{A}} \\
& \mathrm{Q}_{3}=\int_{-\ell_{\mathrm{r}} / 2}^{\ell_{\mathrm{r}} / 2} \int_{\mathrm{A}_{\mathrm{r}}} \sigma \varepsilon_{, 3} \mathrm{dA} \mathrm{A}_{\mathrm{r}} \mathrm{d} \mathrm{x}_{\mathrm{r}} \quad \text { ou } \\
& \mathrm{Q}_{3}=\int_{-\ell_{\mathrm{r}} / 2}^{\ell_{\mathrm{r}} / 2} \int_{\mathrm{A}_{\mathrm{r}}} \sigma\left[\left(1+\frac{\mathrm{q}_{1}}{\ell_{\mathrm{r}}}\right)\left(\frac{2 \mathrm{q}_{3}}{15}-\frac{\mathrm{q}_{2}}{30}\right)-\mathrm{y}_{\mathrm{r}}\left(\frac{6 \mathrm{x}_{\mathrm{r}}}{\ell_{\mathrm{r}}^{2}}+\frac{1}{\ell_{\mathrm{r}}}\right)\right] \mathrm{dA}_{\mathrm{r}} \mathrm{dx} \quad \text { ou } \\
& \mathrm{Q}_{3}=\int_{-\ell_{\mathrm{r}} / 2}^{\ell_{\mathrm{r}} / 2}\left[\left(1+\frac{\mathrm{q}_{1}}{\ell_{\mathrm{r}}}\right)\left(\frac{2 \mathrm{q}_{3}}{15}-\frac{\mathrm{q}_{2}}{30}\right) \mathrm{N}+\left(\frac{6 \mathrm{x}_{\mathrm{r}}}{\ell_{\mathrm{r}}^{2}}+\frac{1}{\ell_{\mathrm{r}}}\right) \mathrm{M}\right] \mathrm{dx_{ \textrm {r } }} \text { ou } \\
& \mathrm{Q}_{3}=\mathrm{N} \ell_{\mathrm{r}}\left(1+\frac{\mathrm{q}_{1}}{\ell_{\mathrm{r}}}\right)\left(\frac{2 \mathrm{q}_{3}}{15}-\frac{\mathrm{q}_{2}}{30}\right)+\frac{4 \mathrm{EI}}{\ell_{\mathrm{r}}} \mathrm{q}_{3}+\frac{2 \mathrm{EI}}{\ell_{\mathrm{r}}} \mathrm{q}_{2} \quad \text { ou } \\
& \mathrm{Q}_{2}=\mathrm{N} \ell_{\mathrm{c}}\left(\frac{2 \mathrm{q}_{3}}{15}-\frac{\mathrm{q}_{2}}{30}\right)+\mathrm{M}_{\mathrm{B}}
\end{aligned}
$$

onde $\mathrm{N}$ é a força normal na seção, neste caso constante ao longo do elemento, $M_{A}$ e $M_{B}$ são os momentos fletores nas extremidades $A$ e $B$ do elemento, na convenção clássica, isto é, tração nas fibras inferiores positivo. A força normal de tração é positiva. 
Os elementos da matriz H são obtidos a partir da equação 3.168 e com o auxílio das equações 3.225 a 3.233 , assim tem-se:

$$
\begin{aligned}
& \mathrm{H}_{11}=\int_{-\ell_{\mathrm{r}} / 2}^{\ell_{\mathrm{r}} / 2} \int_{\mathrm{A}_{\mathrm{r}}} \sigma \varepsilon_{, 11} \mathrm{~d} \mathrm{~A}_{\mathrm{r}} \mathrm{dx} \mathrm{x}_{\mathrm{r}}=0 \\
& \mathrm{H}_{12}=\int_{-\ell_{\mathrm{r}} / 2}^{\ell_{\mathrm{r}} / 2} \int_{\mathrm{A}_{\mathrm{r}}} \sigma \varepsilon_{, 12} \mathrm{dA}_{\mathrm{r}} \mathrm{dx}_{\mathrm{r}}=\int_{-\ell_{\mathrm{r}} / 2}^{\ell_{\mathrm{r}} / 2} \int_{\mathrm{A}_{\mathrm{r}}} \sigma\left[\frac{1}{\ell_{\mathrm{r}}}\left(\frac{2 \mathrm{q}_{2}}{15}-\frac{\mathrm{q}_{3}}{30}\right)\right] \mathrm{dA}_{\mathrm{r}} \mathrm{dx} \mathrm{x}_{\mathrm{r}} \\
& \mathrm{H}_{12}=\int_{-\ell_{\mathrm{r}} / 2}^{\ell_{\mathrm{r}} / 2} \mathrm{~N}\left[\frac{1}{\ell_{\mathrm{r}}}\left(\frac{2 \mathrm{q}_{2}}{15}-\frac{\mathrm{q}_{3}}{30}\right)\right] \mathrm{dx}_{\mathrm{r}} \text { ou } \\
& \mathrm{H}_{12}=\mathrm{N}\left(\frac{2 \mathrm{q}_{2}}{15}-\frac{\mathrm{q}_{3}}{30}\right) \\
& \mathrm{H}_{13}=\int_{-\ell_{\mathrm{r}} / 2}^{\ell_{\mathrm{r}} / 2} \int_{\mathrm{A}_{\mathrm{r}}} \sigma \varepsilon_{, 13} \mathrm{dA}_{\mathrm{r}} \mathrm{dx}_{\mathrm{r}}=\int_{-\ell_{\mathrm{r}} / 2}^{\ell_{\mathrm{r}} / 2} \int_{\mathrm{A}_{\mathrm{r}}} \sigma\left[\frac{1}{\ell_{\mathrm{r}}}\left(\frac{2 \mathrm{q}_{3}}{15}-\frac{\mathrm{q}_{2}}{30}\right)\right] \mathrm{dA}_{\mathrm{r}} \mathrm{dx}_{\mathrm{r}} \\
& \mathrm{H}_{13}=\int_{-\ell_{\mathrm{r}} / 2}^{\ell_{\mathrm{r}} / 2} \mathrm{~N}\left[\frac{1}{\ell_{\mathrm{r}}}\left(\frac{2 \mathrm{q}_{3}}{15}-\frac{\mathrm{q}_{2}}{30}\right)\right] \mathrm{dx}_{\mathrm{r}} \text { ou } \\
& \mathrm{H}_{13}=\mathrm{N}\left(\frac{2 \mathrm{q}_{3}}{15}-\frac{\mathrm{q}_{2}}{30}\right) \\
& \mathrm{H}_{21}=\mathrm{H}_{12} \\
& H_{22}=\int_{-\ell_{\mathrm{r}} / 2}^{\ell_{\mathrm{r}} / 2} \int_{\mathrm{A}_{\mathrm{r}}} \sigma \varepsilon_{, 22} \mathrm{dA}_{\mathrm{r}} \mathrm{dx}_{\mathrm{r}}=\int_{-\ell_{\mathrm{r}} / 2}^{\ell_{\mathrm{r}} / 2} \int_{\mathrm{A}_{\mathrm{r}}} \sigma \frac{2}{15} \lambda \mathrm{dA}_{\mathrm{r}} \mathrm{dx}_{\mathrm{r}} \quad \text { ou }
\end{aligned}
$$




$$
\begin{aligned}
& \mathrm{H}_{22}=\int_{-\ell_{\mathrm{r}} / 2}^{\ell_{\mathrm{r}} / 2} \frac{2 \mathrm{~N}}{15} \lambda \mathrm{dx} \mathrm{x}_{\mathrm{r}}=\frac{2 \mathrm{~N}}{15} \lambda \ell_{\mathrm{r}} \text {, mas } \lambda \ell_{\mathrm{r}}=\ell_{\mathrm{c}} \text {, então } \\
& \mathrm{H}_{22}=\frac{2 \mathrm{~N}}{15} \ell_{\mathrm{c}} \\
& \mathrm{H}_{23}=\int_{-\ell_{\mathrm{r}} / 2}^{\ell_{\mathrm{r}} / 2} \int_{\mathrm{A}_{\mathrm{r}}} \sigma \varepsilon_{, 23} \mathrm{dA}_{\mathrm{r}} \mathrm{dx}_{\mathrm{r}}=\int_{-\ell_{\mathrm{r}} / 2}^{\ell_{\mathrm{r}} / 2} \int_{\mathrm{A}_{\mathrm{r}}} \sigma\left(-\frac{\lambda}{30}\right) \mathrm{dA}_{\mathrm{r}} \mathrm{dx} \text {, ou } \\
& \mathrm{H}_{23}=\int_{-\ell_{\mathrm{r}} / 2}^{\ell_{\mathrm{r}} / 2}-\frac{\mathrm{N} \lambda}{30} \mathrm{dx} \mathrm{x}_{\mathrm{r}}=-\frac{\mathrm{N} \lambda}{30} \ell_{\mathrm{r}} \quad \text { ou } \\
& \mathrm{H}_{23}=-\frac{\mathrm{N} \ell_{\mathrm{c}}}{30} \\
& \mathrm{H}_{31}=\mathrm{H}_{13} \\
& \mathrm{H}_{32}=\mathrm{H}_{23} \\
& \mathrm{H}_{33}=\int_{-t_{\mathrm{r}} / 2}^{t_{\mathrm{r}} / 2} \int_{\mathrm{A}_{\mathrm{r}}} \sigma \varepsilon_{, 33} \mathrm{dA} \mathrm{A}_{\mathrm{r}} \mathrm{dx} \mathrm{x}_{\mathrm{r}}=\int_{-t_{\mathrm{r}} / 2}^{\ell_{\mathrm{r}} / 2} \int_{\mathrm{A}_{\mathrm{r}}} \sigma \frac{2}{15} \lambda \mathrm{dA}_{\mathrm{r}} \mathrm{dx} \quad \text { ou } \\
& \mathrm{H}_{33}=\int_{-\ell_{\mathrm{r}} / 2}^{\ell_{\mathrm{r}} / 2} \frac{2 \mathrm{~N}}{15} \lambda \mathrm{dx}_{\mathrm{r}}=\frac{2 \mathrm{~N}}{15} \lambda \cdot \ell_{\mathrm{r}} \text { ou } \\
& \mathrm{H}_{33}=\frac{2 \mathrm{~N}}{15} \ell_{。}
\end{aligned}
$$

então a matriz $\mathbf{H}$ fica: 


$$
\mathbf{H}=\left[\begin{array}{ccc}
0 & \mathrm{~N}\left(\frac{2 \mathrm{q}_{2}}{15}-\frac{\mathrm{q}_{3}}{30}\right) & \mathrm{N}\left(\frac{2 \mathrm{q}_{3}}{15}-\frac{\mathrm{q}_{2}}{30}\right) \\
\frac{2 \mathrm{~N} \ell_{\mathrm{c}}}{15} & -\frac{\mathrm{N} \ell_{\mathrm{c}}}{30} \\
\text { simetrica } & \frac{2 \mathrm{~N} \ell_{\mathrm{c}}}{15}
\end{array}\right]
$$

Os elementos da matriz D são obtidos a partir da equação 3.169, com auxílio das equações 3.222 a 3.224 e com $\mathrm{D}=\mathrm{E}$ tem-se:

$$
\begin{aligned}
& D_{11}=\int_{-\ell_{r} / 2}^{\ell_{\mathrm{r}} / 2} \int_{\mathrm{A}_{\mathrm{r}}} \mathrm{D} \varepsilon_{, 1} \varepsilon_{, 1} \mathrm{dA}_{\mathrm{r}} \mathrm{dx}_{\mathrm{r}}=\int_{-\ell_{\mathrm{r}} / 2}^{\ell_{\mathrm{r}} / 2} \mathrm{E}_{,, 1}^{2} \int_{\mathrm{A}_{\mathrm{r}}} \mathrm{dA}_{\mathrm{r}} \\
& \mathrm{D}_{11}=\int_{-\ell_{\mathrm{r}} / 2}^{\ell_{\mathrm{r}} / 2} \mathrm{EA} \mathrm{A}_{\mathrm{r}}\left[\frac{1}{\ell_{\mathrm{r}}}+\frac{1}{\ell_{\mathrm{r}}}\left(\frac{\mathrm{q}_{2}^{2}}{15}+\frac{\mathrm{q}_{3}^{2}}{15}-\frac{\mathrm{q}_{2} \mathrm{q}_{3}}{30}\right)\right]^{2} \mathrm{~d} \mathrm{x}_{\mathrm{r}} \\
& \mathrm{D}_{11}=\frac{\mathrm{EA} \mathrm{A}_{\mathrm{r}}}{\ell_{\mathrm{r}}}\left(1+\frac{\mathrm{q}_{2}^{2}}{15}+\frac{\mathrm{q}_{3}^{2}}{15}-\frac{\mathrm{q}_{2} \mathrm{q}_{3}}{30}\right)^{2}
\end{aligned}
$$

Analogamente, obtém-se:

$$
\begin{aligned}
& D_{12}=E_{r}\left(1+\frac{q_{1}}{\ell_{r}}\right)\left(\frac{2 q_{2}}{15}-\frac{q_{3}}{30}\right)\left(1+\frac{q_{2}^{2}}{15}+\frac{q_{3}^{2}}{15}-\frac{q_{2} q_{3}}{30}\right) \\
& D_{13}=E_{r}\left(1+\frac{q_{1}}{\ell_{r}}\right)\left(\frac{2 q_{3}}{15}-\frac{q_{2}}{30}\right)\left(1+\frac{q_{2}^{2}}{15}+\frac{q_{3}^{2}}{15}-\frac{q_{2} q_{3}}{30}\right) \\
& D_{21}=D_{12}
\end{aligned}
$$




$$
\begin{aligned}
& \mathrm{D}_{22}=\mathrm{EA}_{\mathrm{r}} \ell_{\mathrm{c}}\left(1+\frac{\mathrm{q}_{1}}{\ell_{\mathrm{r}}}\right)\left(\frac{2 \mathrm{q}_{2}}{15}-\frac{\mathrm{q}_{3}}{30}\right)+\frac{4 \mathrm{EI}}{\ell_{\mathrm{r}}} \\
& \mathrm{D}_{23}=\mathrm{EA} \mathrm{A}_{\mathrm{r}} \ell_{\mathrm{c}}\left(1+\frac{\mathrm{q}_{1}}{\ell_{\mathrm{r}}}\right)\left(\frac{2 \mathrm{q}_{2}}{15}-\frac{\mathrm{q}_{3}}{30}\right)\left(\frac{2 \mathrm{q}_{3}}{15}-\frac{\mathrm{q}_{2}}{30}\right)+\frac{2 \mathrm{EI}}{\ell_{\mathrm{r}}} \\
& \mathrm{D}_{31}=\mathrm{D}_{13} \\
& \mathrm{D}_{32}=\mathrm{D}_{23} \\
& \mathrm{D}_{33}=\mathrm{E} \mathrm{A} \mathrm{A}_{\mathrm{r}} \ell_{\mathrm{c}}\left(1+\frac{\mathrm{q}_{1}}{\ell_{\mathrm{r}}}\right)\left(\frac{2 \mathrm{q}_{3}}{15}-\frac{\mathrm{q}_{2}}{30}\right)^{2}+\frac{4 \mathrm{EI}}{\ell_{\mathrm{r}}}
\end{aligned}
$$

Se desprezarmos todos os termos multiplicados por $q_{2}$ ou $q_{3}$ por serem suficientemente pequenos, a matriz $\mathbf{D}$ fica:

$$
\mathbf{D}=\left[\begin{array}{ccc}
\frac{\mathrm{EA}_{\mathrm{r}}}{\ell_{\mathrm{r}}} & 0 & 0 \\
& \frac{4 \mathrm{EI}}{\ell \mathrm{r}} & \frac{2 \mathrm{EI}}{\ell \mathrm{r}} \\
& \text { simetrica } & \frac{4 \mathrm{EI}}{\ell \mathrm{r}}
\end{array}\right]
$$

A matriz de rigidez constitutiva do elemento, no sistema local cartesiano, é dada por:

$$
\overline{\mathbf{k}}_{\mathrm{M}}=\overline{\mathbf{B}}^{\mathbf{t}} \mathbf{D} \overline{\mathbf{B}}
$$

onde $\overline{\mathbf{B}}$ é dada por (3.64).

Efetuando-se o triplo produto matricial, obtém-se: 


$$
\overline{\mathbf{k}}_{\mathrm{M}}=\left[\begin{array}{cccccc}
\frac{\mathrm{EA}}{\ell_{\mathrm{r}}} & 0 & 0 & -\frac{\mathrm{EA}_{\mathrm{r}}}{\ell_{\mathrm{r}}} & 0 & 0 \\
& \frac{12 \mathrm{EI}}{\ell_{\mathrm{r}} \ell_{\mathrm{c}}^{2}} & \frac{6 \mathrm{EI}}{\ell_{\mathrm{r}} \ell_{\mathrm{c}}} & 0 & -\frac{12 \mathrm{EI}}{\ell_{\mathrm{r}} \ell_{\mathrm{c}}^{2}} & \frac{6 \mathrm{EI}}{\ell_{\mathrm{r}} \ell_{\mathrm{c}}} \\
& & \frac{4 \mathrm{EI}}{\ell_{\mathrm{r}}} & 0 & -\frac{6 \mathrm{EI}}{\ell_{\mathrm{r}} \ell_{\mathrm{c}}} & \frac{2 \mathrm{EI}}{\ell_{\mathrm{r}}} \\
& & & \frac{\mathrm{EA}}{\ell_{\mathrm{r}}} & 0 & 0 \\
& & & & \frac{12 \mathrm{EI}}{\ell_{\mathrm{r}} \ell_{\mathrm{c}}^{2}} & -\frac{6 \mathrm{EI}}{\ell_{\mathrm{r}} \ell_{\mathrm{c}}} \\
& & & & & \frac{4 \mathrm{EI}}{\ell_{\mathrm{r}}}
\end{array}\right]
$$

que difere da matriz constitutiva tradicional pela presença do produto $\ell_{\mathrm{r}} \ell_{\mathrm{c}}$ ao invés de $\ell_{\mathrm{r}} \ell_{\mathrm{r}}$.

A matriz de rigidez geométrica do elemento, no sistema local cartesiano, é dada por:

$$
\overline{\mathbf{k}}_{\mathrm{G}}=\overline{\mathbf{B}}^{\mathrm{t}} \mathbf{H} \overline{\mathbf{B}}+\mathrm{Q}_{1} \overline{\mathbf{G}}_{1}+\mathrm{Q}_{2} \overline{\mathbf{G}}_{2}+\mathrm{Q}_{3} \overline{\mathbf{G}}_{3}
$$

onde $\overline{\mathbf{G}}_{1}, \overline{\mathbf{G}}_{2}$ e $\overline{\mathbf{G}}_{3}$ são dados por (3.120) e (3.121).

Efetuando-se as operações matriciais indicadas acima, obtém-se: 


$$
\overline{\mathbf{k}}_{\mathrm{G}}=\left[\begin{array}{cccccc}
0 & \frac{\mathrm{V}}{\ell_{\mathrm{c}}} & 0 & 0 & -\frac{\mathrm{V}}{\ell_{\mathrm{c}}} & 0 \\
& \frac{6 \mathrm{~N}}{5 \ell_{\mathrm{c}}} & \frac{\mathrm{N}}{10} & -\frac{\mathrm{V}}{\ell_{\mathrm{c}}} & -\frac{6}{5} \frac{\mathrm{N}}{\ell_{\mathrm{c}}} & \frac{\mathrm{N}}{10} \\
& & \frac{2 \mathrm{~N} \ell_{\mathrm{c}}}{15} & 0 & -\frac{\mathrm{N}}{10} & \frac{\mathrm{N} \ell_{\mathrm{c}}}{30} \\
& & 0 & \frac{\mathrm{V}}{\ell_{\mathrm{c}}} & 0 \\
& & & \frac{6}{5} \frac{\mathrm{N}}{\ell_{\mathrm{c}}} & -\frac{\mathrm{N}}{10} \\
& & & & \frac{2 \mathrm{~N} \ell_{\mathrm{c}}}{15}
\end{array}\right]
$$

onde $V=\frac{M_{B}-M_{A}}{\ell_{c}}$ é a força cortante no elemento.

A matriz de rigidez tangente do elemento em regime elástico linear, no sistema de coordenadas cartesianas locais, quando se adota as aproximações de $2^{\underline{a}}$ ordem e as simplificações citadas, é dada por:

$$
\overline{\mathbf{k}}=\overline{\mathbf{k}}_{\mathrm{M}}+\overline{\mathbf{k}}_{\mathrm{G}}
$$

onde $\overline{\mathbf{k}}_{\mathrm{M}}$ é dada por (3.258) e $\overline{\mathbf{k}}_{\mathrm{G}}$ por (3.260).

\subsection{3 - Elemento Prismático em Regime Elasto-plástico}

Admitindo-se um comportamento elasto-plástico do material, a relação constitutiva incremental é dada por:

$$
\mathrm{d} \sigma=\mathrm{D} d \varepsilon
$$

mas, com:

$$
\begin{aligned}
& \mathrm{D}=\mathrm{D}^{\mathrm{e}} \quad \text { se } \quad \varepsilon \mathrm{d} \varepsilon<0 \\
& \mathrm{D}=\mathrm{D}^{\mathrm{ep}} \quad \text { se } \quad \varepsilon \mathrm{d} \varepsilon>0
\end{aligned}
$$


onde $\mathrm{D}^{\mathrm{e}}$ é o módulo de rigidez tangente elástico e $\mathrm{D}^{\mathrm{ep}}$ é o módulo de rigidez tangente elasto-plástico.

A equação (3.262a) indica um comportamento elástico quando a fibra está em descarga, isto é, quando o incremento de deformação é contrário à deformação.

A equação (3.262b) indica um comportamento elasto-plástico quando a fibra está em carga, isto é, quando o incremento de deformação tem o mesmo sinal da deformação.

Normalmente, toda barra em regime elasto-plástico apresenta uma região dentro da qual o regime é elástico, assim numa seção transversal podese ter algumas fibras em regime elástico e outras em regime elasto-plástico.

Os esforços solicitantes na seção do elemento em regime elasto-plástico serão:

$$
\mathrm{N}=\int_{\mathrm{A}_{\mathrm{r}}} \mathrm{D} \varepsilon \mathrm{dA} \mathrm{A}_{\mathrm{r}}=\int_{\mathrm{A}_{\mathrm{r}}} \mathrm{D}\left(\bar{\varepsilon}_{\mathrm{m}}-\mathrm{y}_{\mathrm{r}} \alpha^{\prime}\right) \mathrm{d} \mathrm{A}_{\mathrm{r}}
$$

admitindo-se $\bar{\varepsilon}_{\mathrm{m}}$ constante, vem:

$$
N=\bar{\varepsilon}_{m} \int_{A_{r}} D d A_{r}-\alpha^{\prime} \int_{A_{r}} D y_{r} d A_{r}
$$

com auxílio de (3.213) e (3.214) vem:

$$
\mathrm{N}=\mathrm{C}_{1} \bar{\varepsilon}_{\mathrm{m}}-\mathrm{C}_{2} \alpha^{\prime}
$$

Cabe observar que neste caso, $N$ não é constante, uma vez que $C_{2} \neq 0$ e $\alpha^{\prime}$ varia linearmente com $\mathbf{x}_{\mathrm{r}}$.

Explicitando-se vem: 


$$
\mathrm{N}=\mathrm{C}_{1} \bar{\varepsilon}_{\mathrm{m}}-\mathrm{C}_{2}\left[\mathrm{q}_{2}\left(\frac{6 \mathrm{x}_{\mathrm{r}}}{\ell_{\mathrm{r}}}-\frac{1}{\ell_{\mathrm{r}}}\right)+\mathrm{q}_{3}\left(\frac{6 \mathrm{x}_{\mathrm{r}}}{\ell_{\mathrm{r}}}+\frac{1}{\ell_{\mathrm{r}}}\right)\right]
$$

e os valores nas extremidades são dados por:

$$
\begin{gathered}
\mathrm{x}_{\mathrm{r}}=-\frac{\ell_{\mathrm{r}}}{2} \Rightarrow \mathrm{N}_{\mathrm{A}}=\mathrm{C}_{\mathrm{IA}} \bar{\varepsilon}_{\mathrm{m}}-\frac{\mathrm{C}_{2 \mathrm{~A}}}{\ell_{\mathrm{r}}}\left(4 \mathrm{q}_{2}+2 \mathrm{q}_{3}\right) \\
\mathrm{x}_{\mathrm{r}}=\frac{\ell_{\mathrm{r}}}{2} \Rightarrow \mathrm{N}_{\mathrm{B}}=\mathrm{C}_{1 \mathrm{~B}} \bar{\varepsilon}_{\mathrm{m}}-\frac{\mathrm{C}_{2 \mathrm{~B}}}{\ell_{\mathrm{r}}}\left(2 \mathrm{q}_{2}+4 \mathrm{q}_{3}\right) \\
\mathrm{M}=-\int_{\mathrm{A}_{\mathrm{r}}} \sigma \mathrm{y}_{\mathrm{r}} \mathrm{dA}=-\int_{\mathrm{A}_{\mathrm{r}}} \mathrm{D} \varepsilon \mathrm{y}_{\mathrm{r}} \mathrm{dA}=-\int_{\mathrm{A}_{\mathrm{r}}} \mathrm{D}\left(\bar{\varepsilon}_{\mathrm{m}}-\mathrm{y}_{\mathrm{r}} \alpha^{\prime}\right) \mathrm{dA}_{\mathrm{r}} \text { ou } \\
\mathrm{M}=-\bar{\varepsilon}_{\mathrm{m}} \int_{\mathrm{A}_{\mathrm{r}}} \mathrm{Dy_{ \textrm {r } }} \mathrm{dA}_{\mathrm{r}}+\alpha^{\prime} \int_{\mathrm{A}_{\mathrm{r}}} \mathrm{Dy_{r } ^ { 2 }} \mathrm{dA}_{\mathrm{r}}
\end{gathered}
$$

com o auxílio de (3.214) e (3.215) resulta:

$$
M=-C_{2} \bar{\varepsilon}_{m}+C_{3} \alpha^{\prime}
$$

Explicitando-se vem:

$$
M=-C_{2} \bar{\varepsilon}_{m}+C_{3}\left[q_{3}\left(\frac{6 x_{r}}{\ell_{r}^{2}}-\frac{1}{\ell_{r}}\right)+q_{3}\left(\frac{6 x_{r}}{\ell_{r}^{2}}+\frac{1}{\ell_{r}}\right)\right]
$$

e os valores nas extremidades são dados por:

$$
\mathrm{x}_{\mathrm{r}}=-\frac{\ell_{\mathrm{r}}}{2} \Rightarrow \mathrm{M}_{\mathrm{A}}=-\mathrm{C}_{2 \mathrm{~A}} \bar{\varepsilon}_{\mathrm{m}}-\frac{\mathrm{C}_{3 \mathrm{~A}}}{\ell_{\mathrm{r}}}\left(4 \mathrm{q}_{2}+2 \mathrm{q}_{3}\right)
$$




$$
\mathrm{x}_{\mathrm{r}}=\frac{\ell_{\mathrm{r}}}{2} \Rightarrow \mathrm{M}_{\mathrm{B}}=-\mathrm{C}_{2 \mathrm{~B}} \bar{\varepsilon}_{\mathrm{m}}-\frac{\mathrm{C}_{3 \mathrm{~B}}}{\ell_{\mathrm{r}}}\left(2 \mathrm{q}_{2}+4 \mathrm{q}_{3}\right)
$$

Cabe observar que $C_{1}, C_{2}$ e $C_{3}$ são constantes na seção mas variam ao longo de $\mathrm{x}_{\mathrm{r}}$, em função da variação de $\mathrm{D}$.

Para simplificar admite-se que os elementos sejam curtos de maneira que $\mathrm{N}, \mathrm{C}_{1}, \mathrm{C}_{2}$ e $\mathrm{C}_{3}$ sejam constantes ao longo do elemento e iguais ao seu valor no centro do elemento, dados por:

$$
\begin{aligned}
& \mathrm{C}_{1 \mathrm{~m}}=\frac{\mathrm{C}_{1 \mathrm{~A}}+\mathrm{C}_{1 \mathrm{~B}}}{2} \\
& \mathrm{C}_{2 \mathrm{~m}}=\frac{\mathrm{C}_{2 \mathrm{~A}}+\mathrm{C}_{2 \mathrm{~B}}}{2} \\
& \mathrm{C}_{3 \mathrm{~m}}=\frac{\mathrm{C}_{3 \mathrm{~A}}+\mathrm{C}_{3 \mathrm{~B}}}{2}
\end{aligned}
$$

e que os momentos fletores tenham uma distribuição linear, como no regime elástico linear. Assim, os valores de $\mathrm{Q}_{\alpha}$, desprezando-se os termos multiplicados por $\mathrm{q}_{2}$ e $\mathrm{q}_{3}$, são dados a partir das equações 3.234 a 3.236, por:

$$
\begin{aligned}
& \mathrm{Q}_{1}=\frac{\mathrm{N}_{\mathrm{A}}+\mathrm{N}_{\mathrm{B}}}{2}=\mathrm{N}_{\mathrm{m}} \\
& \mathrm{Q}_{2}=-\mathrm{M}_{\mathrm{A}} \\
& \mathrm{Q}_{3}=\mathrm{M}_{\mathrm{B}}
\end{aligned}
$$

Os elementos da matriz H serão análogos aos obtidos no regime elástico linear (eq. 3.246), bastando substituir $\mathrm{N}$ por $\mathrm{N}_{\mathrm{m}}$. 
Os elementos da matriz D são obtidos a partir da equações 3.207 a 3.212, aplicando-se as mesmas simplificações adotadas para o elemento no regime elástico linear, isto é, admitir-se $\bar{\varepsilon}=\bar{\varepsilon}_{\mathrm{m}}$ e desprezar-se os termos multiplicados por $\mathrm{q}_{2}$ e $\mathrm{q}_{3}$.

Sendo assim, a matriz D para o elemento prismático em regime elastoplástico será:

$$
\mathbf{D}=\left[\begin{array}{ccc}
\frac{\mathrm{C}_{1 \mathrm{~m}}}{\ell_{\mathrm{r}}} & \frac{\mathrm{C}_{2 \mathrm{~m}}}{\ell_{\mathrm{r}}} & -\frac{\mathrm{C}_{2 \mathrm{~m}}}{\ell_{\mathrm{r}}} \\
& \frac{4 \mathrm{C}_{3 \mathrm{~m}}}{\ell_{\mathrm{r}}} & \frac{2 \mathrm{C}_{3 \mathrm{~m}}}{\ell_{\mathrm{r}}} \\
\text { simétrica } & & \frac{4 \mathrm{C}_{3 \mathrm{~m}}}{\ell_{\mathrm{r}}}
\end{array}\right]
$$

Comparando-se esta matriz com a matriz $\mathbf{D}$ do caso elástico (eq. 3.256), observa-se a presença dos elementos $\frac{\mathrm{C}_{2 \mathrm{~m}}}{\ell_{\mathrm{r}}} \mathrm{e}-\frac{\mathrm{C}_{2 \mathrm{~m}}}{\ell_{\mathrm{r}}}$ que eram sempre nulos em 3.256, e podem ser não nulos no caso elasto-plástico devido à variação da rigidez tangente " $D$ " na seção.

A matriz de rigidez constitutiva do elemento em regime elasto-plástico, no sistema local cartesiano, é obtida através da equação 3.257 , e vale:

$$
\overline{\mathbf{k}}_{\mathrm{M}}=\left[\begin{array}{cccccc}
\frac{\mathrm{C}_{1 \mathrm{~m}}}{\ell_{\mathrm{r}}} & 0 & -\frac{\mathrm{C}_{2 \mathrm{~m}}}{\ell_{\mathrm{r}}} & -\frac{\mathrm{C}_{1 \mathrm{~m}}}{\ell_{\mathrm{r}}} & 0 & \frac{\mathrm{C}_{2 \mathrm{~m}}}{\ell_{\mathrm{r}}} \\
& \frac{12 \mathrm{C}_{3 \mathrm{~m}}}{\ell_{\mathrm{r}} \ell_{\mathrm{c}}^{2}} & \frac{6 \mathrm{C}_{3 \mathrm{~m}}}{\ell_{\ell_{\mathrm{c}}}} & 0 & -\frac{12 \mathrm{C}_{3 \mathrm{~m}}}{\ell_{\mathrm{r}} \ell_{\mathrm{c}}^{2}} & \frac{6 \mathrm{C}_{3 \mathrm{~m}}}{\ell_{\mathrm{r}} \ell_{\mathrm{c}}} \\
& & \frac{4 \mathrm{C}_{3 \mathrm{~m}}}{\ell_{\mathrm{r}}} & \frac{\mathrm{C}_{2 \mathrm{~m}}}{\ell_{\mathrm{r}}} & -\frac{6 \mathrm{C}_{3 \mathrm{~m}}}{\ell_{\mathrm{r}} \ell_{\mathrm{c}}} & \frac{2 \mathrm{C}_{3 \mathrm{~m}}}{\ell_{\mathrm{r}}} \\
& & & \frac{\mathrm{C}_{1 \mathrm{~m}}}{\ell_{\mathrm{r}}} & 0 & -\frac{\mathrm{C}_{2 \mathrm{~m}}}{\ell_{\mathrm{r}}} \\
& & & & \frac{12 \mathrm{C}_{3 \mathrm{~m}}}{\ell_{\mathrm{r}} \ell_{\mathrm{c}}^{2}} & -\frac{6 \mathrm{C}_{3 \mathrm{~m}}}{\ell_{\mathrm{r}} \ell_{\mathrm{c}}} \\
& \text { simétrica } & & & & \frac{4 \mathrm{C}_{3 \mathrm{~m}}}{\ell_{\mathrm{r}}}
\end{array}\right]
$$


A matriz de rigidez geométrica do elemento em regime elasto-plástico, no sistema local cartesiano, é obtida efetuando-se as operações matriciais indicadas na equação 3.259 , resultando-se:

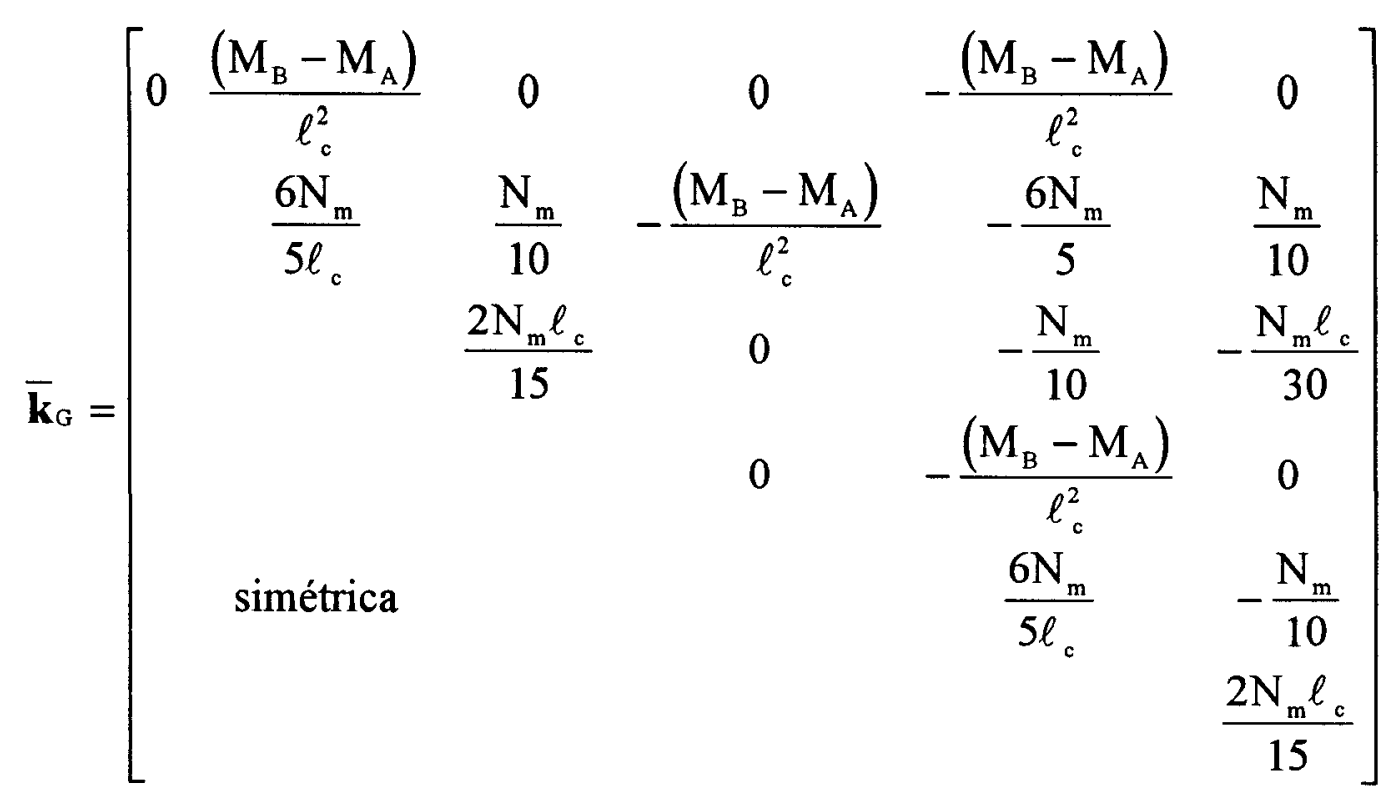

onde $\mathrm{N}_{\mathrm{m}}, \mathrm{M}_{\mathrm{A}}$ e $\mathrm{M}_{\mathrm{B}}$ são dados pelas equações $3.274,3.269$ e 3.270 .

Analogamente ao caso elástico, a matriz de rigidez tangente do elemento, no sistema cartesiano local é dada pela eq. 3.261, isto é,

$$
\overline{\mathbf{k}}=\overline{\mathbf{k}}_{\mathrm{M}}+\overline{\mathbf{k}}_{\mathrm{G}}
$$

\subsection{Equilíbrio Estrutural}

A compatibilidade entre os deslocamentos nodais da estrutura e os do elemento, ambos descritos em coordenadas-deslocamento cartesianas, é feita através da relação: 


$$
\mathbf{p}=\mathbf{A} \mathbf{r}
$$

onde $\mathbf{A}$ é a matriz de incidência cinemática, $\mathbf{p}$ é o vetor de deslocamentos nodais do elemento e $\mathbf{r}$ é o vetor dos deslocamentos nodais da estrutura. Adotando-se o sistema de cartesiano local paralelo ao sistema cartesiano global, esta matriz A conterá apenas 0 e 1, sendo portanto independente da atual configuração da estrutura.

Assim, variando-se a equação 3.280 , obtém-se:

$$
\delta \mathbf{p}=\mathbf{A} \delta \mathbf{r}
$$

O trabalho virtual interno da estrutura é obtido pela somatória dos trabalhos virtuais internos de seus elementos. Assim, pode-se escrever:

$$
\begin{aligned}
& \delta \mathrm{W}_{\mathrm{I}}=\sum_{\mathrm{i}=1}^{\mathrm{ne}} \mathbf{P}_{\mathrm{i}}^{\mathrm{T}}\left(\delta \mathbf{p}_{\mathrm{i}}\right)_{\mathrm{i}} \quad \text { ou } \\
& \delta \mathrm{W}_{\mathrm{I}}=\sum_{\mathrm{i}=1}^{\mathrm{ne}} \mathbf{P}_{\mathrm{i}}^{\mathrm{T}} \mathbf{A}_{\mathrm{i}} \delta \mathbf{r}
\end{aligned}
$$

onde ne é o número de elementos da estrutura.

A partir dos vetores dos esforços nodais internos no elemento e aplicando-se o princípio da contragradiência obtém-se a contribuição destes esforços no vetor dos esforços internos na estrutura. Somando-se a contribuição de todos os elementos, obtém-se o vetor dos esforços internos na estrutura, dado por:

$$
\mathbf{S}=\sum_{i=1}^{n e} \mathbf{A}_{i}^{T} \mathbf{P}_{i}
$$


Levando-se (3.283) em (3.282), resulta:

$$
\delta \mathrm{W}_{\mathrm{I}}=\mathbf{S}^{\mathrm{T}} \delta \mathbf{r}
$$

onde $\mathbf{S}$ é o vetor dos esforços internos na estrutura, que será função do nível de tensão " $\sigma$ " e dos deslocamentos " $r$ ".

Supondo-se que a estrutura só está submetida a carregamento aplicado nos nós, o trabalho virtual externo é dado por:

$$
\delta \mathrm{W}_{\mathrm{E}}=\mathbf{R}^{\mathrm{T}} \delta \mathbf{r}
$$

onde $\mathbf{R}$ é o vetor dos esforços nodais externos na estrutura.

Aplicando-se o Princípio dos Trabalhos Virtuais, tem-se que para um deslocamento virtual qualquer $\delta$, pode-se igualar $\delta \mathrm{W}_{\mathrm{I}}=\delta \mathrm{W}_{\mathrm{E}}$, resultando:

$$
\begin{aligned}
& \mathbf{S}^{\mathrm{T}}=\mathbf{R}^{\mathrm{T}} \quad \text { ou } \\
& \mathbf{S}=\mathbf{R}
\end{aligned}
$$

que é a equação de equilíbrio estrutural.

\subsection{Equilíbrio Incremental}

A partir da equação (3.286), pode-se obter a equação de equilibrio incremental como:

$$
\Delta \mathbf{S}=\Delta \mathbf{R}
$$

sendo $\Delta \mathbf{S}$ obtido do espalhamento dos vetores $\Delta \mathbf{P}_{\mathrm{i}}$ ou seja: 


$$
\Delta \mathbf{S}=\sum_{\mathrm{i}=1}^{\mathrm{ne}} \mathbf{A}_{\mathrm{i}}^{\mathrm{T}} \Delta \mathbf{P}_{\mathrm{i}}
$$

onde $\Delta \mathbf{P}_{\mathrm{i}}$ é o incremento de esforços nodais no elemento $i$ e pode ser expresso matricialmente como:

$$
\Delta \mathbf{P}_{\mathrm{i}}=\mathbf{k}_{\mathrm{i}} \Delta \mathbf{p}_{\mathrm{i}}
$$

sendo $\Delta \mathrm{p}_{\mathrm{i}} \mathbf{o}$ incremento de deslocamentos nodais do elemento $i$ e $\mathbf{k}_{\mathrm{i}}$ a matriz de rigidez tangente do elemento $i$.

Substituindo-se (3.289) em (3.288) obtém-se:

$$
\Delta \mathbf{S}=\sum_{\mathrm{i}=1}^{\mathrm{ne}} \mathbf{A}_{\mathrm{i}}^{\mathrm{T}} \mathbf{k}_{\mathrm{i}} \Delta \mathbf{p}_{\mathrm{i}}
$$

mas de (3.280) vem:

$$
\Delta \mathbf{p}_{\mathrm{i}}=\mathbf{A}_{\mathrm{i}} \Delta \mathbf{r}
$$

Levando-se (3.291) em (3.290) vem:

$$
\begin{aligned}
& \Delta \mathbf{S}=\sum_{\mathrm{i}=1}^{\text {ne }} \mathbf{A}_{\mathrm{i}}^{\mathrm{T}} \mathbf{k}_{\mathrm{i}} \mathbf{A}_{\mathrm{i}} \Delta \mathbf{r} \quad \text { ou } \\
& \Delta \mathbf{S}=\mathbf{K} \Delta \mathbf{r}
\end{aligned}
$$

onde $\mathbf{K}=\sum_{\mathrm{i}=1}^{\text {ne }} \mathbf{A}_{\mathrm{i}}^{\mathrm{T}} \mathbf{k}_{\mathrm{i}} \mathbf{A}_{\mathrm{i}}$ é a matriz de rigidez tangente da estrutura, obtida da contribuição das matrizes de rigidez dos elementos através das matrizes de incidência cinemática $\mathbf{A}_{\mathbf{i}}$ dos elementos. 
Assim, a equação (3.287) do equilíbrio incremental pode ser escrita da seguinte forma:

$$
\Delta \mathbf{R}=\mathbf{K} \Delta \mathbf{r}
$$

onde $\Delta \mathbf{r}$ é o vetor de incremento de deslocamentos na estrutura, associado ao incremento de forças nodais externos $\Delta \mathbf{R}$.

\subsection{Solução do Problema Estático}

Sendo o vetor dos esforços nodais internos $\mathbf{S}$ função dos deslocamentos nodais $\mathbf{r}$ a equação de equilíbrio estrutural (eq. 3.286) se transforma em:

$$
\mathbf{S}(\mathrm{r})-\mathbf{R}=\mathbf{0}
$$

onde 0 é o vetor nulo. A equação matricial acima representa um sistema de equações não-lineares em $\mathbf{r}$, que por conseguinte, é dependente de $\mathbf{p}$ e $\sigma$ nos elementos.

\subsubsection{Métodos de Solução de Equações Não-lineares}

Os métodos de solução numérica da equação de equilíbrio não-linear têm tido um desenvolvimento constante. Não serão aqui tratados os aspectos envolvidos na solução das equações não-lineares de equilíbrio. DESAI \& ABEL (1972), BOYLE \& JENNINGS (1973), BATHE \& CIMENTO (1980), CRISFIELD (1980), BATHE \& DVORKIN (1983), OWEN E GOMES (1984), PROENÇA (1989), ARAÚJO (1989), CILONI (1993) e BATHE (1996) discutem estes aspectos destacando vantagens, desvantagens e mostrando algumas técnicas para a otimização dos métodos supracitados. 
Neste trabalho foi utilizada a forma incremental do método iterativo de Newton-Raphson que é uma das formas mais utilizadas e conhecidas de solução de problemas de equilíbrio não-linear. Como foi implementada, é possível especificar o número de incrementos de carga, recaindo na forma iterativa, ou o número de iterações de cada incremento, de modo a poder degenerar para a forma apenas incremental. O motivo da escolha se deve ao fato do método de Newton-Raphson ter convergência mais rápida que os demais métodos.

\subsection{2 - Método Iterativo de Newton-Raphson}

A seguir apresenta-se o método iterativo de Newton-Raphson de uma maneira formal.

Deseja-se encontrar a solução das equações

$$
\mathbf{f}\left(\mathbf{r}^{*}\right)={ }^{t+\Delta t} \mathbf{R}\left(\mathbf{r}^{*}\right)-{ }^{t+\Delta t} \mathbf{S}\left(\mathbf{r}^{*}\right)=\mathbf{0}
$$

Admitindo-se que numa solução iterativa tenha-se determinado ${ }^{\mathrm{l}+\Delta t} \mathbf{r}^{(i-1)}$, então uma expansão em série de Taylor dá:

$$
\begin{aligned}
\mathbf{f}\left(r^{*}\right)= & \mathbf{f}\left({ }^{t+\Delta t} \mathbf{r}^{(i-1)}\right)+\left.\left[\frac{\partial \mathbf{f}}{\partial \mathbf{r}}\right]\right|_{t+\Delta t} \mathbf{r}^{(i-1)}\left(\mathbf{r}^{*}{ }^{t+\Delta t} \mathbf{r}^{(i-1)}\right)+ \\
& + \text { termos de ordem superior }
\end{aligned}
$$

substituindo-se (3.295) em (3.296), e admitindo-se $\mathbf{R}$ independente dos deslocamentos nodais, tem-se

$$
{ }^{t+\Delta t} \mathbf{R}-{ }^{t+\Delta t} \mathbf{S}+\left[\frac{\partial \mathbf{S}}{\partial \mathbf{r}}\right] \|^{t+\Delta t} \mathbf{r}^{(i-1)}+\text { termos de ordem superior }=\mathbf{0}
$$


Desprezando-se os termos de ordem superior, resulta:

$$
{ }^{t+\Delta t} \mathbf{K}^{(i-1)} \Delta \mathbf{r}^{(i)}={ }^{t+\Delta t} \mathbf{R}-{ }^{t+\Delta t} \mathbf{S}^{(i-1)}
$$

onde ${ }^{t+\Delta t} \mathbf{K}^{(i-1)}$ é a matriz de rigidez da iteração $(i-I)$ e dado por:

$$
\left.{ }^{t+\Delta t} \mathbf{K}^{(i-1)}=\left[\frac{\partial \mathbf{S}}{\partial \mathbf{r}}\right]\right]_{t+\Delta t} \mathbf{r}^{(i-1)}
$$

e os deslocamentos atualizados dos pontos nodais na iteração atual $i$ é:

$$
{ }^{t+\Delta t} \mathbf{r}^{(i)}={ }^{i+\Delta t} \mathbf{r}^{(i-1)}+\Delta \mathbf{r}^{(i)}
$$

Estas equações constituem a base do método de Newton-Raphson, que é ilustrado na Fig. 3.9.

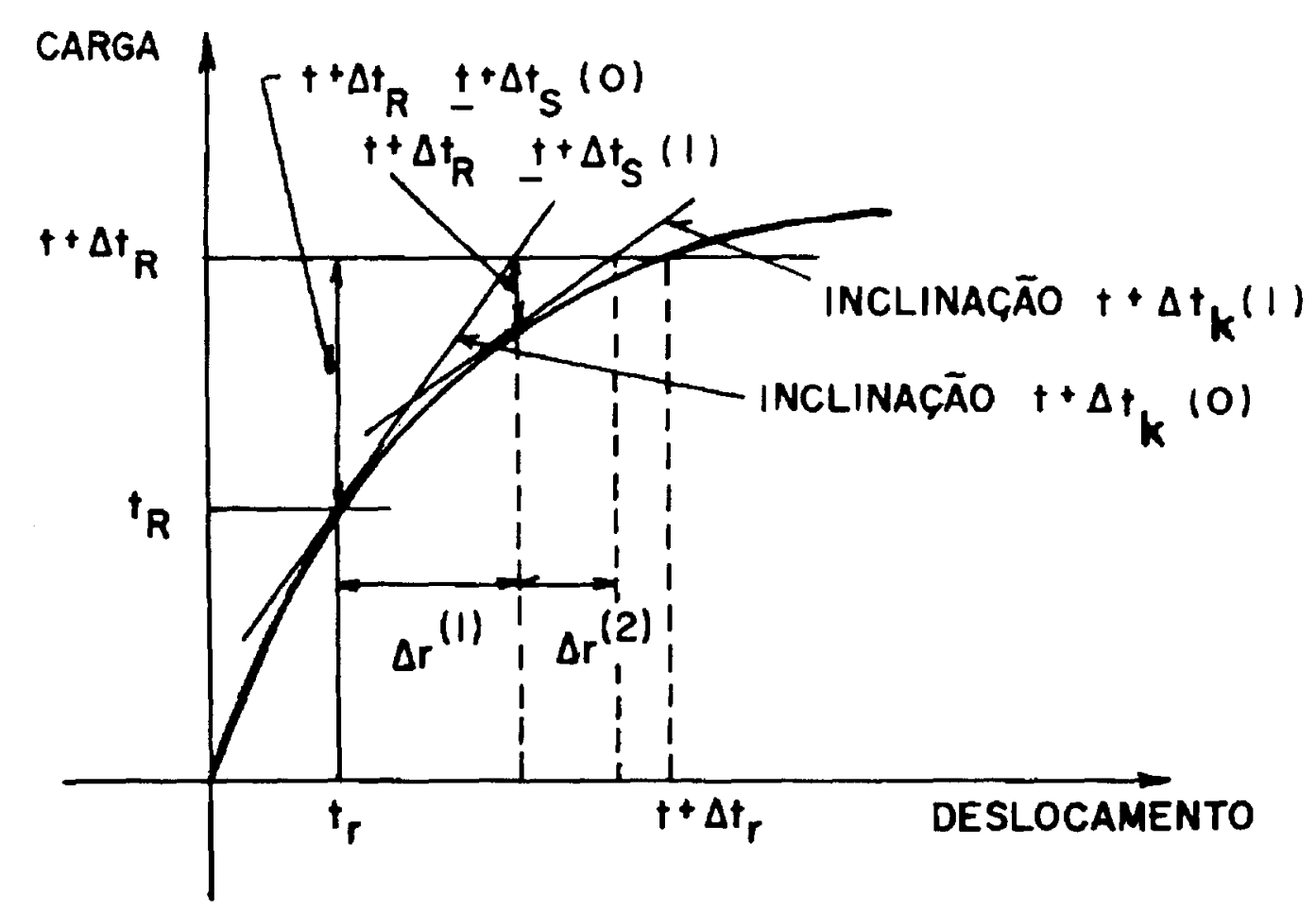

FIGURA 3.9- Método iterativo de Newton-Raphson 
Uma característica deste método iterativo é que uma nova matriz de rigidez é calculada em cada iteração, e por isso é conhecido por método Newton-Raphson puro. Esta necessidade de reavaliar a matriz de rigidez em cada iteração pode gerar custos computacionais elevados. Procurando-se reduzir esses custos, várias alternativas são possíveis, as quais são consideradas como modificações do método original.

Uma dessas alternativas é a que usa a matriz de rigidez inicial ${ }^{\circ} \mathbf{K} \mathrm{em}$ (3.308) e assim a equação fica:

$$
{ }^{\circ} \mathbf{K}^{(\mathrm{i}-1)} \Delta \mathbf{r}^{(\mathrm{i})}={ }^{\mathrm{t}+\Delta t} \mathbf{R}-{ }^{\mathrm{t}+\Delta t} \mathbf{S}^{(\mathrm{i}-1)}
$$

com condições iniciais dadas por:

$$
{ }^{t+\Delta t} \mathbf{S}^{(0)}={ }^{\mathrm{t}} \mathbf{S} ; \quad{ }^{\mathrm{t}+\Delta \mathrm{t}} \mathbf{r}^{(0)}={ }^{\mathrm{t}} \mathbf{r}
$$

neste caso, apenas a matriz ${ }^{\circ} \mathrm{K}$ necessita ser triangularizada, evitando-se assim cálculos extensos na reavaliação dos coeficientes de rigidez e na triangularização da matriz de rigidez quando se usa (3.297). Assim, este processo corresponde à linearização da resposta sobre a configuração inicial da estrutura e pode convergir mais lentamente ou mesmo divergir. Este método é conhecido como método de Newton-Raphson com rigidez inicial. A Fig. 3.10 ilustra este método.

Uma outra alternativa que tem se mostrado a mais eficiente entre os métodos Newton-Raphson combina os dois métodos anteriores e é chamado método de Newton-Raphson modificado. Neste método usa-se:

$$
{ }^{\tau} \mathbf{K} \Delta \mathbf{r}^{(i)}={ }^{t+\Delta t} \mathbf{R}-{ }^{1+\Delta t} \mathbf{S}^{(i-1)}
$$

com condições iniciais dadas por: 


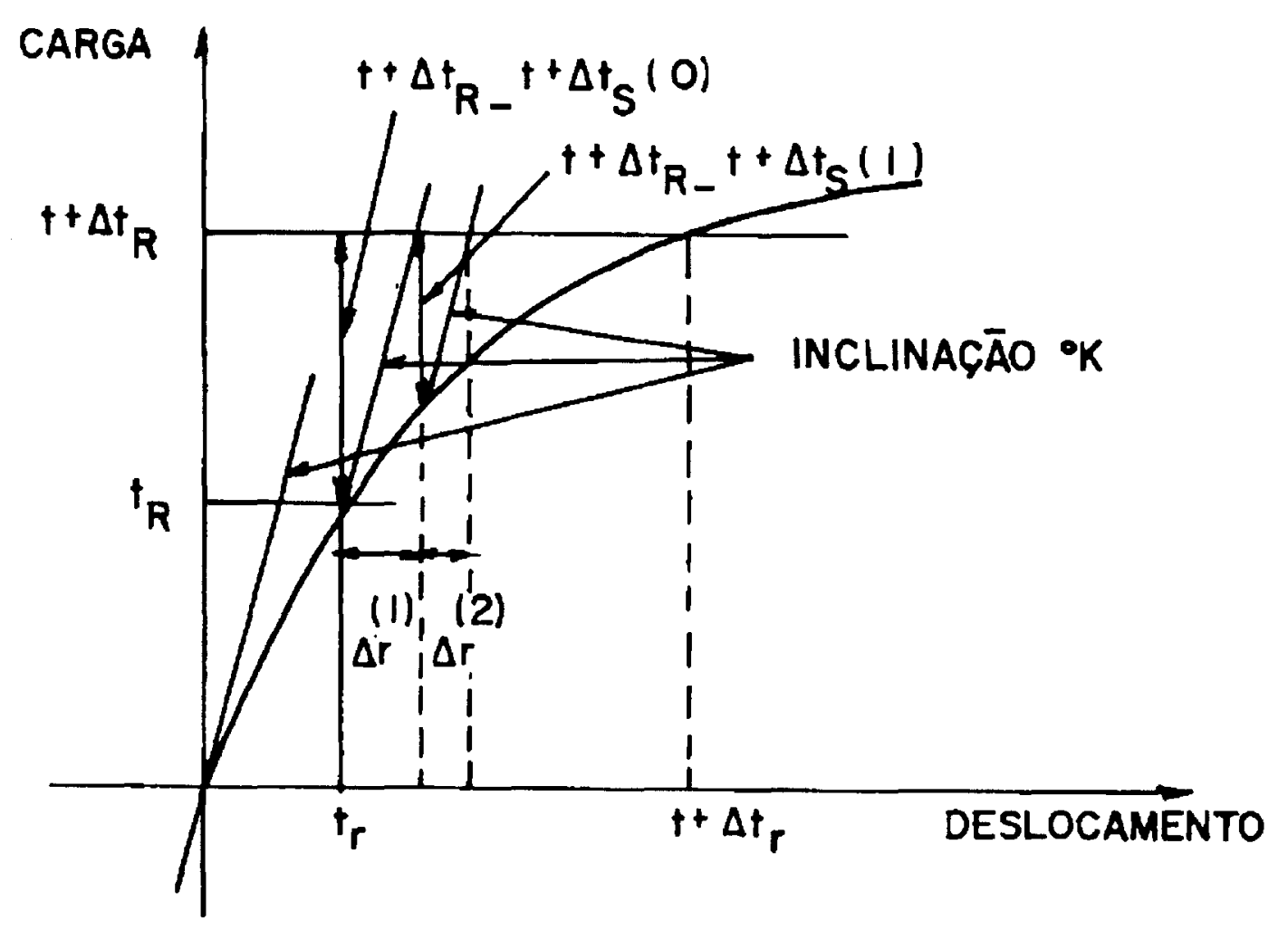

FIGURA 3.10 - Método de Newton-Raphson com rigidez inicial

$$
{ }^{t+\Delta t} \mathbf{S}^{(0)}={ }^{\mathrm{t}} \mathbf{S} ; \quad{ }^{\mathrm{t}+\Delta t} \mathbf{r}^{(0)}={ }^{\mathrm{t}} \mathbf{r}
$$

onde $\tau$ corresponde a uma das reconhecidas configurações de equilíbrio para os instantes $0, \Delta \mathrm{t}, 2 \Delta \mathrm{t}, \ldots$ ou $t$. Este método envolve menos reformulações da matriz de rigidez que o método puro e se baseia na matriz de rigidez atualizada numa configuração de equilíbrio conhecida. A escolha de quais instantes em que se atualizará a matriz de rigidez dependerá do grau de não-linearidade da estrutura, ou seja, estrutura de comportamento pouco não-linear necessitará de poucas atualizações da matriz de rigidez. Este processo está ilustrado na Fig. 3.11, admitindo-se atualização da matriz de rigidez apenas no início de cada incremento. 


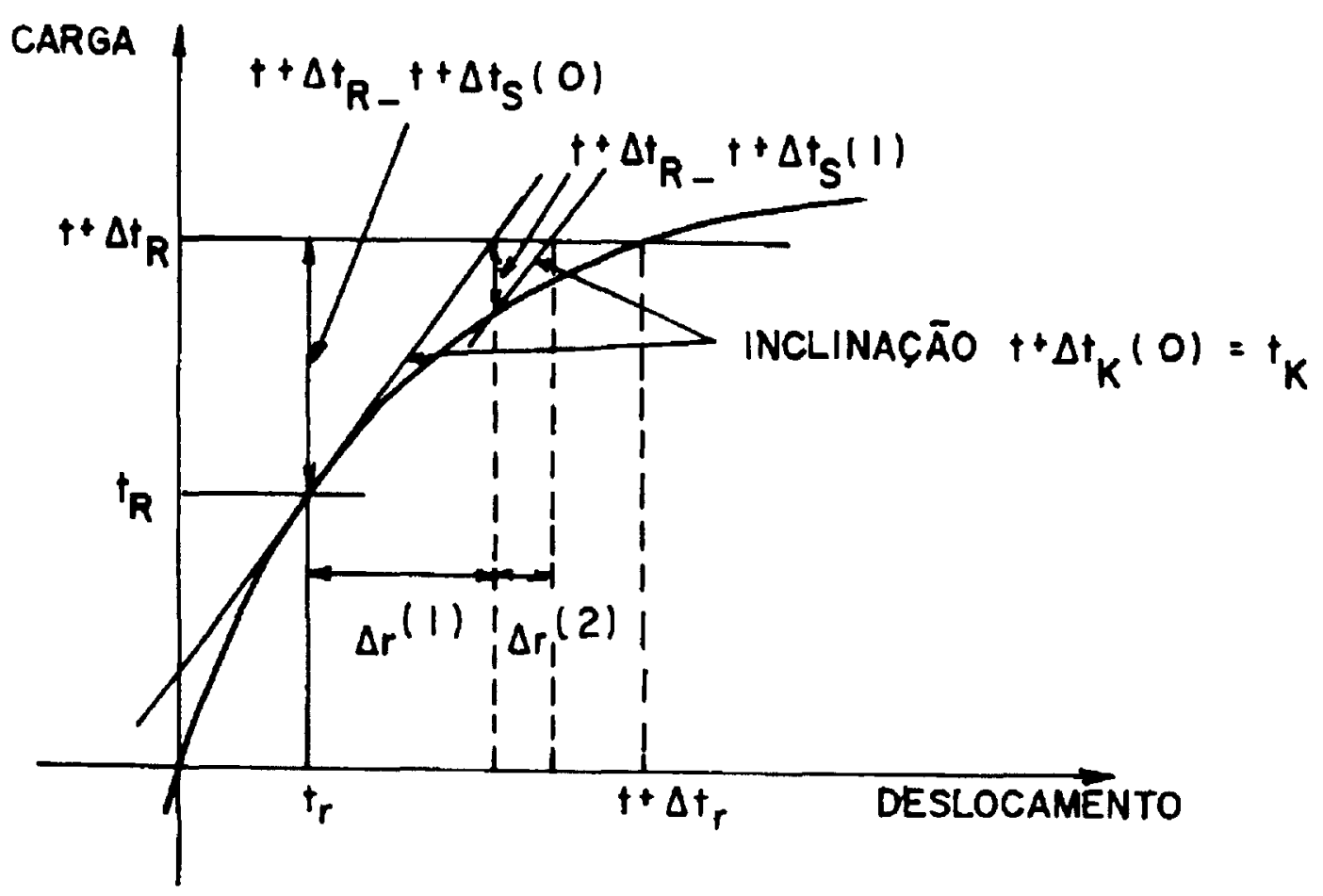

FIGURA 3. 11 - Método de Newton-Raphson modificado

Segundo BATHE (1996) as principais propriedades do método de Newton-Raphson são:

1- Se a matriz de rigidez tangente ${ }^{\mathrm{t}+\Delta t} \mathbf{K}^{(\mathrm{i}-1)}$ é não singular, se f e suas derivadas primeira em relação às variáveis solução são contínuas numa vizinhança de $\mathbf{r}^{*}$, e se ${ }^{t+\Delta t} \mathbf{r}^{(i-1)}$ leva àquela vizinhança, então ${ }^{t+\Delta t} \mathbf{r}^{(i)}$ será mais próximo de $\mathbf{r}^{*}$ que ${ }^{t+\Delta t} \mathbf{r}^{(i-1)}$ e a sequência de soluções iterativas geradas pelas equações (3.297) a (3.299) converge para $\mathbf{r}^{*}$.

2- Se a matriz de rigidez também satisfaz

$$
\left\|{ }^{t+\Delta t} \mathbf{K}\left|\mathbf{r}_{1}-{ }^{t+\Delta t} \mathbf{K}\right| r_{2}\right\| \leq L\left\|\mathbf{r}_{1}-\mathbf{r}_{2}\right\|
$$


para todos $\mathbf{r}_{1}$ e $\mathbf{r}_{2}$ na vizinhança de $\mathbf{r}^{*}$ e $L>0$, então a convergência é quadrática. Isto significa que se $o$ erro após a iteração $i$ é de ordem $\varepsilon$, então o erro após a iteração $i+l$ será de ordem $\varepsilon^{2}$.

A consequência prática dessas propriedades é que se a solução da iteração corrente é suficientemente próxima da solução $r^{*}$ e se a matriz de rigidez não muda drasticamente, pode-se esperar uma rápida convergência. Por outro lado, se a solução da iteração corrente não é suficientemente próxima de $\mathbf{r}^{*}$ e/ou a matriz de rigidez exata não é usada e/ou muda drasticamente, a iteração pode divergir.

\subsubsection{Critérios de Convergência}

O uso de métodos iterativos requer critérios apropriados de convergência. Usando-se critérios não apropriados, a iteração pode terminar antes que a precisão necessária tenha sido atingida ou continuar desnecessariamente após a precisão requerida ter sido atingida.

Em geral, os critérios de convergência podem ser fixados em função de: deslocamentos, esforços residuais e energia interna ou trabalho.

Como em análises não-lineares procura-se obter a configuração da estrutura correspondente a um determinado passo de carga, é natural que se pretenda que os deslocamentos no final de cada iteração estejam, dentro de uma tolerância aceitável, próximos aos correspondentes à solução correta.

Então um critério realista de convergência é:

$$
\frac{\left\|\Delta \mathbf{r}^{(i)}\right\|_{2}}{\| t+\Delta t} \|_{2} \times 100 \leq \varepsilon_{D}
$$


onde $\varepsilon_{\mathrm{D}}$ é a tolerância admitida para os deslocamentos em percentagem e $\left\|\Delta \mathbf{r}^{(i)}\right\|_{2}$ é a norma euclidiana do vetor dos incrementos de deslocamentos na iteração $i$ e dada por:

$$
\left\|\Delta \mathbf{r}^{(i)}\right\|_{2}=\left|\sqrt{\sum_{j=1}^{N}\left(\mathbf{r}_{j}^{(i)}\right)^{2}}-\sqrt{\sum_{j=1}^{N}\left(\mathbf{r}_{j}^{(i-1)}\right)^{2}}\right|
$$

e $\left\|^{t+\Delta t} \mathbf{r}\right\|_{2}$ é a norma euclidiana do vetor dos deslocamentos nodais da estrutura obtido no final do incremento. Como esses deslocamentos são desconhecidos, eles devem ser aproximados. Frequentemente, adota-se para estes deslocamentos aqueles obtidos na iteração atual $i$, assim tem-se:

$$
\|t+\Delta t\|_{2}=\left\|^{t+\Delta t} \mathbf{r}^{(i)}\right\|_{2}=\sqrt{\sum_{j=1}^{N}\left(\mathbf{r}_{j}^{(i)}\right)^{2}}
$$

onde $\mathrm{N}$ é o número de graus de liberdade da estrutura.

Conforme salienta BATHE (1996), em algumas análises, a solução real pode estar longe do valor obtido quando a convergência é medida usando ${ }^{t+\Delta t} \mathbf{r}^{(i)}$ no lugar de ${ }^{t+\Delta t} \mathbf{r}$. Isto ocorre quando os deslocamentos mudam pouco em cada iteração mas continuam mudando durante várias iterações.

Um segundo critério, análogo ao anterior mas mais confiável, baseia-se nos esforços residuais, sendo dado por:

$$
\frac{\left\|\Delta \mathbf{R}^{(i)}\right\|_{2}}{\left\|{ }^{t+\Delta t} \mathbf{R}-{ }^{t} \mathbf{S}\right\|_{2}} \times 100 \leq \varepsilon_{\mathrm{F}}
$$


sendo $\left\|\Delta \mathbf{R}^{(\mathrm{i})}\right\|_{2}$ a norma euclidiana dos esforços residuais obtidos no final da iteração $i,\left\|^{t+\Delta t} \mathbf{R}-{ }^{t} \mathbf{S}\right\|_{2}$ a norma euclidiana do incremento de cargas nodais e $\varepsilon_{F}$ é a tolerância admitida para os esforços em percentagem. O problema deste critério é que ele não envolve deslocamentos, assim por exemplo numa análise eslasto-plástica de uma estrutura de material com pequeno módulo de endurecimento (strain hardening), entrando na região plástica, os esforços residuais podem ser muito pequenos enquanto os deslocamentos ainda podem variar muito.

A possibilidade de falha destes dois critérios quando usados separadamente pode ser contornada mediante a utilização de um terceiro critério que envolva tanto deslocamentos quanto esforços. Este critério é baseado na energia incremental interna, e é dado por:

$$
\frac{\Delta \mathbf{r}^{(i)^{t}}\left({ }^{t+\Delta t} \mathbf{R}-{ }^{t+\Delta t} \mathbf{S}^{(i-1)}\right)}{\Delta \mathbf{r}^{(1)^{t}}\left({ }^{(+\Delta t} \mathbf{R}-{ }^{t} \mathbf{S}\right)} \times 100 \leq \varepsilon_{\mathrm{E}}
$$

onde o numerador representa $o$ incremento de energia interna durante a iteração $i$, o denominador representa o incremento de energia interna inicial e $\varepsilon_{\mathrm{E}}$ é a tolerância admitida para a energia interna em percentagem.

As tolerâncias admitidas para a convergência devem ser pequenas, OWEN \& GOMES (1984) adotam tolerâncias de $0,5 \%$, enquanto OWEN \& HINTON (1980) adotam 1,0\%. 


\subsubsection{Análise da Estabilidade}

A análise não-linear incremental permite estudar o comportamento da estrutura em todas as fases de carregamento. Pontos de instabilidade podem ser detectados a partir das curvas carga-deslocamento pela presença de grandes deslocamentos.

Considere um pórtico plano em equilíbrio sob a ação de esforços nodais externos R. Suponha-se que este equilíbrio seja perturbado por um pequeno deslocamento $\delta$ r. Admitindo-se que os esforços nodais externos mantenham-se constantes durante esta perturbação, o trabalho externo realizado por estes esforços é dado por:

$$
\delta \mathrm{W}_{\mathrm{E}}=\mathbf{R}^{\mathrm{t}} \delta \mathbf{r}
$$

O trabalho realizado pelos esforços internos $\mathbf{S}$ durante esta perturbação, é dado até o termo de segunda ordem por:

$$
\delta W_{I}=\mathbf{S}^{t} \delta \mathbf{r}+\frac{1}{2} \delta \mathbf{S}^{t} \delta \mathbf{r}
$$

Levando-se a equação (3.292) em (3.309), resulta:

$$
\delta \mathrm{W}_{\mathrm{I}}=\mathbf{S}^{\mathrm{t}} \delta \mathbf{r}+\frac{1}{2} \delta \mathbf{r}^{\mathrm{t}} \mathbf{K} \Delta \mathbf{r}
$$

A condição para que o equilíbrio seja estável é que, para qualquer perturbação $\underset{\sim}{\mathrm{r}}, \mathrm{o}$ trabalho dos esforços internos seja maior do que o trabalho dos esforços externos, ou seja:

$$
\delta \mathrm{W}_{\mathrm{I}}-\delta \mathrm{W}_{\mathrm{E}}>0
$$


Como na situação de equilíbrio $\mathbf{S}=\mathbf{R}$ (eq. 3.286) e levando-se as equações (3.318 e 3.310$)$ na equação (3.322), obtém-se:

$$
\delta \mathbf{r}^{t} \mathbf{K} \delta \mathbf{r}>0
$$

para qualquer $\delta \mathbf{r} \neq \mathbf{0}$. Assim, uma condição suficiente para que o equilíbrio da estrutura seja estável é que a matriz de rigidez tangente $\mathbf{K}$ seja positiva definida.

Então, a presença de um ponto crítico pode ser detectada monitorandose o sinal dos elementos da diagonal principal da matriz de rigidez tangente decomposta. Um elemento negativo indica a existência de um auto-valor nulo no intervalo de carga que compreende o último passo e o passo atual. $O$ autovetor correspondente representa um modo de deformação sem incremento de carga, caracterizando-se um ponto crítico.

\subsection{5 - Exemplos de Estruturas Elásticas Sujeitas a Grandes Deslocamentos}

A aplicabilidade da formulação apresentada é ilustrada através de exemplos já apresentados por outros pesquisadores. Nestes exemplos, ficam destacadas a eficiência do programa de análise e sua capacidade de seguir corretamente o comportamento não-linear das estruturas analisadas. Com o objetivo de destacar a aplicabilidade do programa na análise de realmente grandes deslocamentos, admitiu-se o comportamento elástico do material. No capítulo seguinte, quando a formulação é aplicada a pórticos de concreto armado, a não-linearidade física é considerada.

Nos exemplos apresentados é destacado ainda o número ideal de elementos necessários para uma precisa representação de grandes deslocamentos. 


\subsubsection{Exemplo 1: Viga em Balanço}

Trata-se de uma viga em balanço sujeita a uma carga vertical concentrada progressiva, cuja solução analítica encontra-se no trabalho de MATTIASSON (1981).

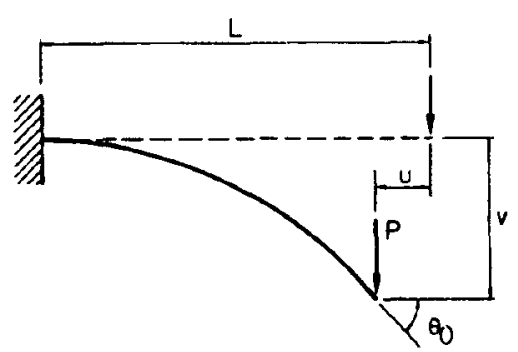

FIGURA 3.12 - Viga em balanço

Foram utilizados 26 incrementos iguais de carga na análise incremental e iterativa com tolerância nos deslocamentos de $0,1 \%$. Com o método de Newton-Raphson puro, a convergência ocorreu com no máximo 5 iterações.

As Tab. 3.1, 3.2 e 3.3 mostram respectivamente os valores dos deslocamentos adimensionais $\mathrm{u} / \ell$ e $\mathrm{v} / \ell$ e das rotações $\theta_{0}$ na extremidade livre da viga (Fig. 3.12), quando se discretiza a barra com 1, 2, 4, 6 ou 10 elementos, assim como os valores obtidos da solução analítica. As curvas carga $x$ deslocamento são mostradas nas Fig. 3.13, 3.14 e 3.15. 
TABELA 3.1 - Valores dos deslocamentos adimensionais $\mathrm{u} / \ell$ na extremidade livre da viga em balanço da Fig. 3.12

\begin{tabular}{|c|c|c|c|c|c|c|}
\hline $\left.\mathbf{P L}^{2}\right) / \mathbf{E I}$ & $\begin{array}{c}\mathbf{1} \\
\text { Elemento }\end{array}$ & $\begin{array}{c}\mathbf{2} \\
\text { Elementos }\end{array}$ & $\begin{array}{c}\mathbf{4} \\
\text { Elementos }\end{array}$ & $\begin{array}{c}\mathbf{6} \\
\text { Elementos }\end{array}$ & $\begin{array}{c}\mathbf{1 0} \\
\text { Elementos }\end{array}$ & Analítico \\
\hline 0.0 & 0.00000 & 0.00000 & 0.00000 & 0.00000 & 0.00000 & 0.00000 \\
\hline 0.2 & 0.00264 & 0.00263 & 0.00263 & 0.00263 & 0.00263 & 0.00265 \\
\hline 0.4 & 0.00953 & 0.01007 & 0.01024 & 0.01027 & 0.01029 & 0.01035 \\
\hline 0.6 & 0.02029 & 0.02173 & 0.02221 & 0.02230 & 0.02235 & 0.02249 \\
\hline 0.8 & 0.03440 & 0.03684 & 0.03769 & 0.03785 & 0.03794 & 0.03817 \\
\hline 1.0 & 0.05123 & 0.05453 & 0.05575 & 0.05598 & 0.05612 & 0.05643 \\
\hline 1.2 & 0.07016 & 0.07397 & 0.07551 & 0.07582 & 0.07599 & 0.07640 \\
\hline 1.4 & 0.09058 & 0.09445 & 0.09625 & 0.09662 & 0.09682 & 0.09732 \\
\hline 1.6 & 0.11197 & 0.11541 & 0.11738 & 0.11780 & 0.11802 & 0.11860 \\
\hline 1.8 & 0.13390 & 0.13642 & 0.13847 & 0.13892 & 0.13916 & 0.13981 \\
\hline 2.0 & 0.15601 & 0.15716 & 0.15921 & 0.15968 & 0.15994 & 0.16064 \\
\hline 2.5 & 0.21073 & 0.20677 & 0.20843 & 0.20890 & 0.20915 & 0.20996 \\
\hline 3.0 & 0.26217 & 0.25189 & 0.25289 & 0.25330 & 0.25354 & 0.25442 \\
\hline 3.5 & 0.30935 & 0.29230 & 0.29246 & 0.29281 & 0.29302 & 0.29394 \\
\hline 4.0 & 0.35213 & 0.32833 & 0.32754 & 0.32780 & 0.32799 & 0.32894 \\
\hline 4.5 & 0.39073 & 0.36047 & 0.35868 & 0.35885 & 0.35901 & 0.35999 \\
\hline 5.0 & 0.42551 & 0.38923 & 0.38642 & 0.38651 & 0.38663 & 0.38763 \\
\hline 5.5 & 0.45689 & 0.41508 & 0.41126 & 0.41125 & 0.41134 & 0.41236 \\
\hline 6.0 & 0.48527 & 0.43842 & 0.43359 & 0.43349 & 0.43356 & 0.43459 \\
\hline 6.5 & 0.51099 & 0.45941 & 0.45378 & 0.45360 & 0.45364 & 0.45468 \\
\hline 7.0 & 0.53439 & 0.47892 & 0.47212 & 0.47185 & 0.47186 & 0.47293 \\
\hline 7.5 & 0.55575 & 0.49661 & 0.48885 & 0.48851 & 0.48860 & 0.48957 \\
\hline 8.0 & 0.57529 & 0.51288 & 0.50419 & 0.50377 & 0.50374 & 0.50483 \\
\hline 8.5 & 0.59324 & 0.52790 & 0.51831 & 0.51781 & 0.51775 & 0.51886 \\
\hline 9.0 & 0.60970 & 0.54181 & 0.53135 & 0.53077 & 0.53069 & 0.53182 \\
\hline 9.5 & 0.62504 & 0.55475 & 0.54344 & 0.54278 & 0.54268 & 0.54383 \\
\hline 10.0 & 0.63917 & 0.56682 & 0.55468 & 0.55395 & 0.55383 & 0.55500 \\
\hline
\end{tabular}


TABELA 3.2 - Valores dos deslocamentos adimensionais $\mathrm{v} / \ell$ na extremidade livre da viga em balanço da Fig. 3.12

\begin{tabular}{|c|c|c|c|c|c|c|}
\hline $\left.\mathbf{P L}^{2}\right) / \mathbf{E I}$ & $\begin{array}{c}\mathbf{1} \\
\text { Elemento }\end{array}$ & $\begin{array}{c}\mathbf{2} \\
\text { Elementos }\end{array}$ & $\begin{array}{c}\mathbf{4} \\
\text { Elementos }\end{array}$ & $\begin{array}{c}\mathbf{6} \\
\text { Elementos }\end{array}$ & $\begin{array}{c}\mathbf{1 0} \\
\text { Elementos }\end{array}$ & Analítico \\
\hline 0.0 & 0.00000 & 0.00000 & 0.00000 & 0.00000 & 0.00000 & 0.00000 \\
\hline 0.2 & 0.06641 & 0.06637 & 0.06637 & 0.06637 & 0.06637 & 0.06636 \\
\hline 0.4 & 0.13156 & 0.13112 & 0.13102 & 0.13100 & 0.13090 & 0.13098 \\
\hline 0.6 & 0.19445 & 0.19285 & 0.19251 & 0.19244 & 0.19242 & 0.19235 \\
\hline 0.8 & 0.25427 & 0.25060 & 0.24980 & 0.24966 & 0.24960 & 0.24945 \\
\hline 1.0 & 0.31046 & 0.30378 & 0.30235 & 0.30210 & 0.30198 & 0.30172 \\
\hline 1.2 & 0.36271 & 0.35222 & 0.34998 & 0.34960 & 0.34942 & 0.34901 \\
\hline 1.4 & 0.41091 & 0.39600 & 0.39284 & 0.39231 & 0.39204 & 0.39147 \\
\hline 1.6 & 0.45511 & 0.43539 & 0.43122 & 0.43053 & 0.43018 & 0.42941 \\
\hline 1.8 & 0.49548 & 0.47075 & 0.46553 & 0.46466 & 0.46423 & 0.46326 \\
\hline 2.0 & 0.53226 & 0.50247 & 0.49619 & 0.49515 & 0.49463 & 0.49346 \\
\hline 2.5 & 0.60966 & 0.56834 & 0.55952 & 0.55807 & 0.55737 & 0.55566 \\
\hline 3.0 & 0.67075 & 0.61937 & 0.60819 & 0.60638 & 0.60550 & 0.60325 \\
\hline 3.5 & 0.71932 & 0.65965 & 0.64633 & 0.64420 & 0.64316 & 0.64039 \\
\hline 4.0 & 0.75835 & 0.69206 & 0.67685 & 0.67443 & 0.67325 & 0.66996 \\
\hline 4.5 & 0.79006 & 0.71865 & 0.70173 & 0.69906 & 0.69777 & 0.69397 \\
\hline 5.0 & 0.81614 & 0.74083 & 0.72239 & 0.71948 & 0.71809 & 0.71379 \\
\hline 5.5 & 0.83783 & 0.75963 & 0.73982 & 0.73670 & 0.73522 & 0.73042 \\
\hline 6.0 & 0.85606 & 0.77578 & 0.75474 & 0.75143 & 0.74985 & 0.74457 \\
\hline 6.5 & 0.87154 & 0.78984 & 0.76767 & 0.76418 & 0.76252 & 0.75676 \\
\hline 7.0 & 0.88479 & 0.80221 & 0.77901 & 0.77534 & 0.77362 & 0.76737 \\
\hline 7.5 & 0.89624 & 0.81320 & 0.78906 & 0.78523 & 0.78343 & 0.77670 \\
\hline 8.0 & 0.90619 & 0.82306 & 0.79804 & 0.79405 & 0.79218 & 0.78498 \\
\hline 8.5 & 0.91493 & 0.83196 & 0.80613 & 0.80199 & 0.80007 & 0.79239 \\
\hline 9.0 & 0.92646 & 0.84006 & 0.81348 & 0.80920 & 0.80721 & 0.79906 \\
\hline 9.5 & 0.92949 & 0.84748 & 0.82021 & 0.81578 & 0.81373 & 0.80510 \\
\hline 10.0 & 0.93561 & 0.85431 & 0.82639 & 0.82183 & 0.81971 & 0.81061 \\
\hline
\end{tabular}


TABELA 3.3 - Valores das rotações $\theta_{0}$ na extremidade livre da viga em balanço da Fig. 3.12

\begin{tabular}{|c|c|c|c|c|c|c|}
\hline $\left.\mathbf{P L}^{2}\right) / \mathbf{E I}$ & $\begin{array}{c}\mathbf{1} \\
\text { Elemento }\end{array}$ & $\begin{array}{c}\mathbf{2} \\
\text { Elementos }\end{array}$ & $\begin{array}{c}\mathbf{4} \\
\text { Elementos }\end{array}$ & $\begin{array}{c}\mathbf{6} \\
\text { Elementos }\end{array}$ & $\begin{array}{c}\mathbf{1 0} \\
\text { Elementos }\end{array}$ & Analítico \\
\hline 0.0 & 0.00000 & 0.00000 & 0.00000 & 0.00000 & 0.00000 & 0.00000 \\
\hline 0.2 & 0.09973 & 0.09965 & 0.09964 & 0.09964 & 0.09964 & 0.09964 \\
\hline 0.4 & 0.19809 & 0.19376 & 0.19721 & 0.19718 & 0.19717 & 0.19716 \\
\hline 0.6 & 0.29391 & 0.29142 & 0.29093 & 0.29084 & 0.29080 & 0.29074 \\
\hline 0.8 & 0.38623 & 0.38158 & 0.37948 & 0.37929 & 0.37920 & 0.37906 \\
\hline 1.0 & 0.47437 & 0.46407 & 0.46209 & 0.46176 & 0.46158 & 0.46135 \\
\hline 1.2 & 0.55789 & 0.54153 & 0.52846 & 0.53793 & 0.53767 & 0.53730 \\
\hline 1.4 & 0.63658 & 0.61297 & 0.60860 & 0.60786 & 0.60749 & 0.60698 \\
\hline 1.6 & 0.71041 & 0.67858 & 0.67279 & 0.67181 & 0.67133 & 0.67065 \\
\hline 1.8 & 0.77947 & 0.73873 & 0.73143 & 0.73020 & 0.72959 & 0.72876 \\
\hline 2.0 & 0.84397 & 0.79383 & 0.78496 & 0.78349 & 0.78275 & 0.78175 \\
\hline 2.5 & 0.98657 & 0.91227 & 0.89954 & 0.89745 & 0.89641 & 0.89500 \\
\hline 3.0 & 1.10673 & 1.00820 & 0.99179 & 0.98912 & 0.98780 & 0.98602 \\
\hline 3.5 & 1.20861 & 1.08674 & 1.06698 & 1.06379 & 1.06222 & 1.06012 \\
\hline 4.0 & 1.29571 & 1.15179 & 1.12905 & 1.12541 & 1.12362 & 1.12124 \\
\hline 4.5 & 1.37083 & 1.20626 & 1.18090 & 1.17687 & 1.17489 & 1.17228 \\
\hline 5.0 & 1.43619 & 1.25231 & 1.22468 & 1.22031 & 1.21817 & 1.21537 \\
\hline 5.5 & 1.49351 & 1.29158 & 1.26199 & 1.25734 & 1.25507 & 1.25211 \\
\hline 6.0 & 1.54416 & 1.32531 & 1.29407 & 1.28918 & 1.28680 & 1.28370 \\
\hline 6.5 & 1.58923 & 1.35449 & 1.32185 & 1.31676 & 1.31428 & 1.31107 \\
\hline 7.0 & 1.62958 & 1.37987 & 1.34607 & 1.34081 & 1.33825 & 1.33496 \\
\hline 7.5 & 1.66591 & 1.40204 & 1.36730 & 1.36191 & 1.35929 & 1.35593 \\
\hline 8.0 & 1.69879 & 1.42151 & 1.38601 & 1.38051 & 1.37784 & 1.37443 \\
\hline 8.5 & 1.72869 & 1.43866 & 1.40258 & 1.39700 & 1.39429 & 1.39084 \\
\hline 9.0 & 1.75600 & 1.45382 & 1.41732 & 1.41167 & 1.40894 & 1.40547 \\
\hline 9.5 & 1.78104 & 1.46726 & 1.43048 & 1.42479 & 1.42030 & 1.41854 \\
\hline 10.0 & 1.80408 & 1.47919 & 1.44227 & 1.43654 & 1.43378 & 1.43029 \\
\hline & & & & & & \\
\hline
\end{tabular}




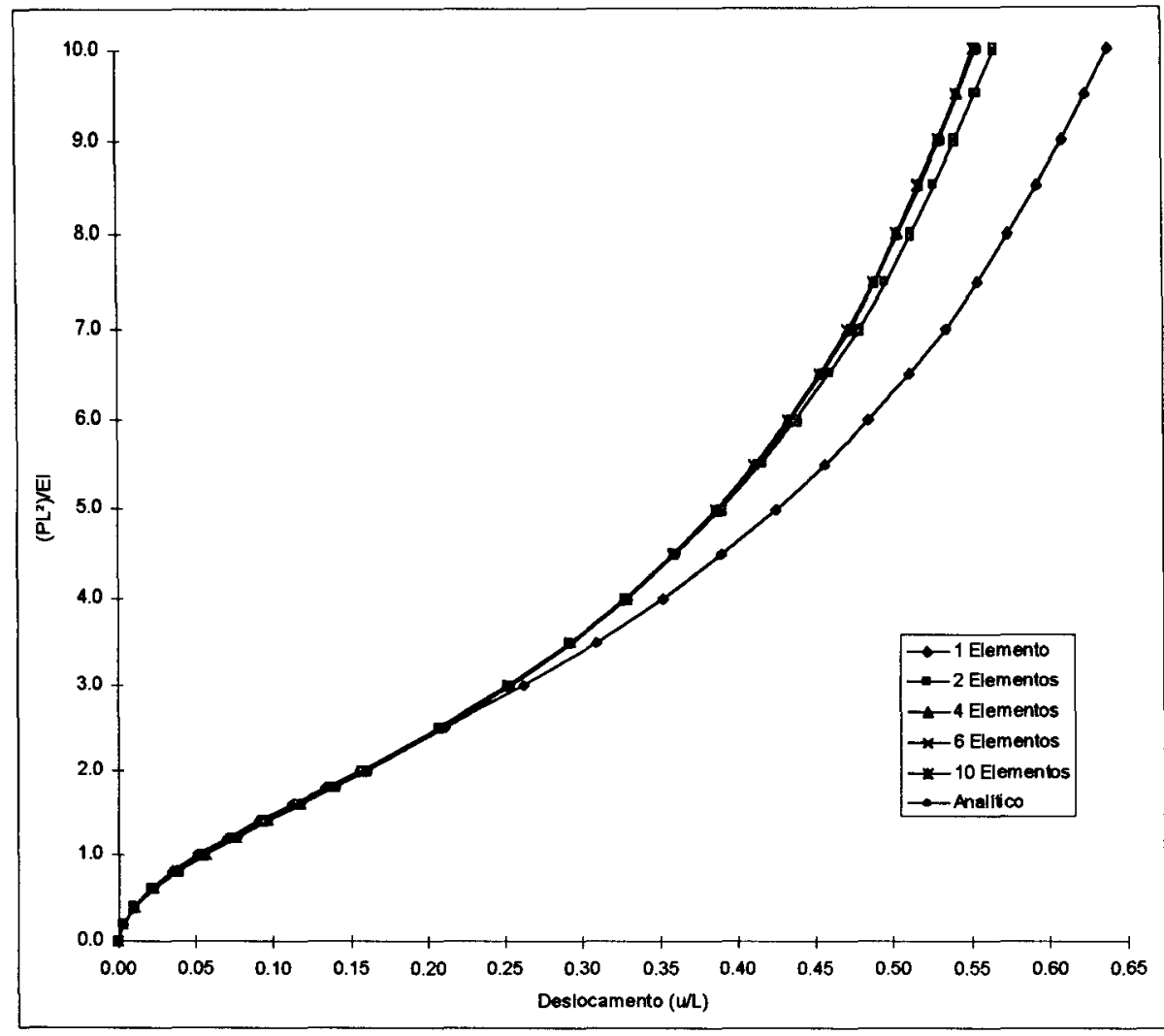

FIGURA 3.13 - Curva carga paramétrica $\mathrm{x}$ deslocamento adimensional $\mathrm{u} / \ell$

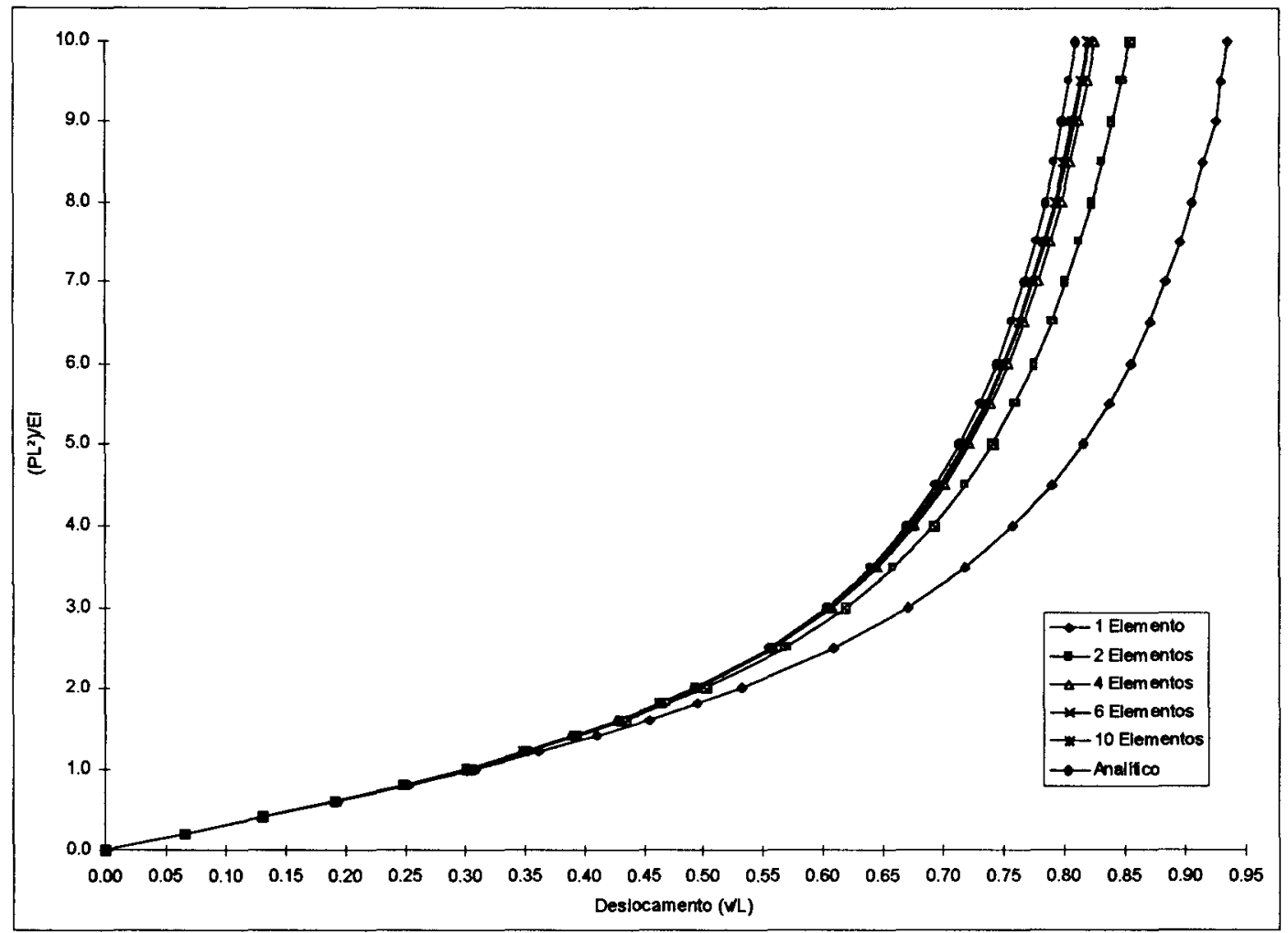

FIGURA 3.14 - Curva carga paramétrica $\mathrm{x}$ deslocamento adimensional $\mathrm{v} / \ell$ 


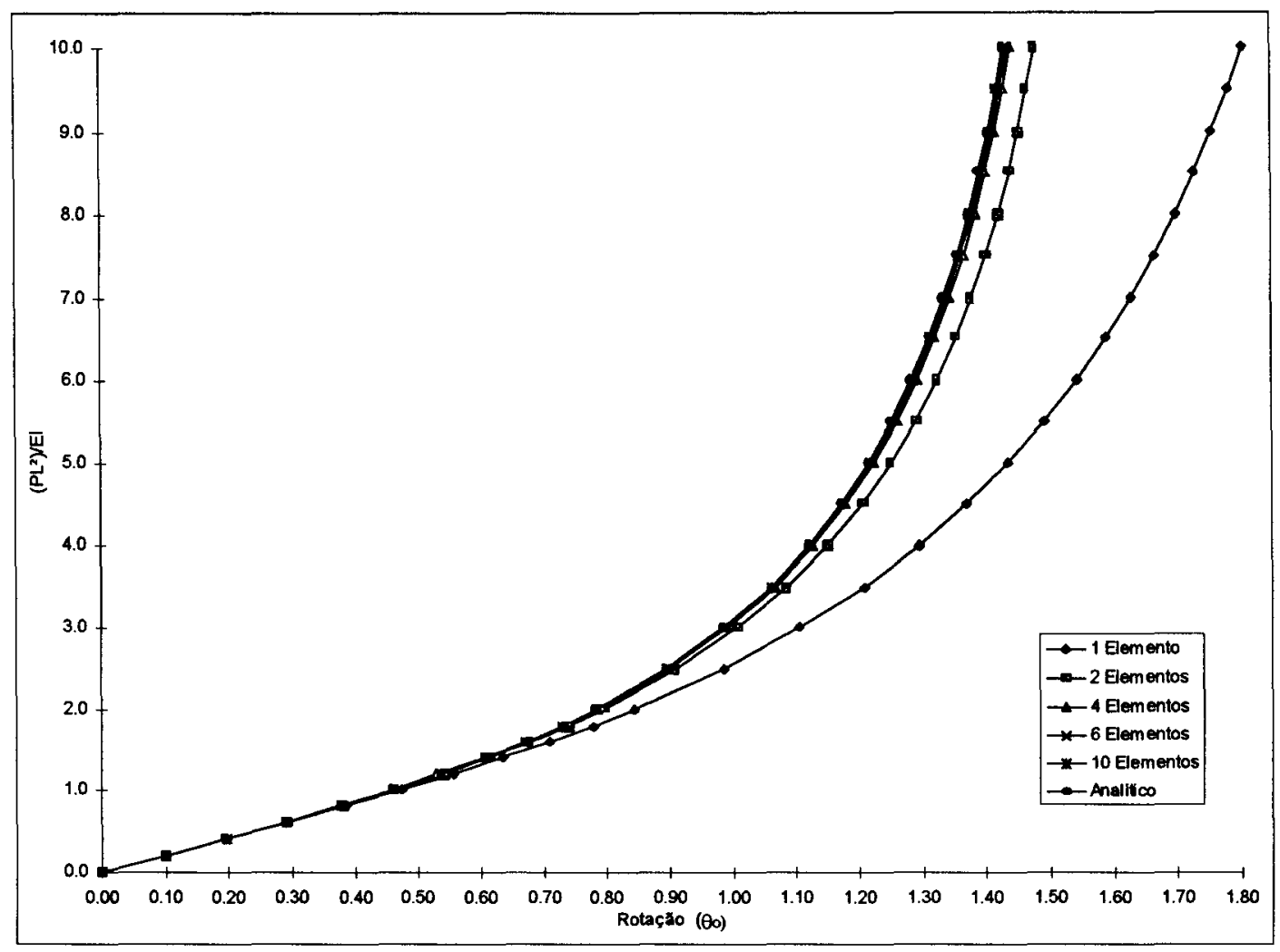

FIGURA 3.15 - Curva carga paramétrica x rotação $\theta_{\mathrm{o}}$

Da Tab. 3.2 pode-se observar que para deslocamentos verticais (v) de até $19 \%$ do vão $(\ell)$ da viga, ou seja, nível de carga igual a $0,6 \mathrm{PL}^{2} / \mathrm{EI}$, os resultados obtidos discretizando-se a barra com 1 elemento são praticamente iguais aos analíticos. Para deslocamentos verticais (v) até $55 \%$ do vão $(\ell)$ da viga, ou seja, nível de carga igual a $2,5 \mathrm{PL}^{2} / \mathrm{EI}$, os resultados obtidos discretizando-se a barra em 2 elementos não diferem em mais de $2,5 \%$ dos resultados analíticos. Discretizando-se a barra em 4 elementos, o erro máximo obtido é da ordem de $2 \%$ para um deslocamento vertical (v) da ordem de $81 \%$ do vão $(\ell)$ e para o nível de carga de $10 \mathrm{PL}^{2} / \mathrm{EI}$.

A mesma viga foi calculada discretizando-se a barra em 10 elementos, aplicando-se agora o carregamento em 10 incrementos iguais e os resultados estão na Tab. 3.4. 
TABELA 3.4 - Valores dos deslocamentos $(u / \ell),(v / \ell)$ e rotações $\theta_{0}$ na extremidade livre da viga com 10 incrementos de carga

\begin{tabular}{|c|c|c|c|c|c|c|}
\cline { 2 - 7 } \multicolumn{1}{c|}{} & \multicolumn{2}{c|}{$\mathrm{u} \ell$} & \multicolumn{2}{c|}{$\mathrm{u} / \ell$} & \multicolumn{2}{c|}{$\theta 0$} \\
\hline$\left(\mathbf{P L}^{2}\right) / \mathbf{E I}$ & $\begin{array}{c}\mathbf{1 0} \\
\text { Elementos }\end{array}$ & Analítico & $\begin{array}{c}\mathbf{1 0} \\
\text { Elementos }\end{array}$ & Analítico & $\begin{array}{c}\mathbf{1 0} \\
\text { Elementos }\end{array}$ & Analítico \\
\hline 0.0 & 0.00000 & 0.00000 & 0.00000 & 0.00000 & 0.00000 & 0.00000 \\
\hline 1.0 & 0.05629 & 0.05643 & 0.30186 & 0.30172 & 0.46150 & 0.46135 \\
\hline 2.0 & 0.16015 & 0.16064 & 0.49440 & 0.49346 & 0.78260 & 0.78175 \\
\hline 3.0 & 0.25374 & 0.25442 & 0.60523 & 0.60325 & 0.98764 & 0.96602 \\
\hline 4.0 & 0.32816 & 0.32894 & 0.67297 & 0.66996 & 1.12348 & 1.12124 \\
\hline 5.0 & 0.38679 & 0.38763 & 0.71779 & 0.71379 & 1.21805 & 1.21537 \\
\hline 6.0 & 0.43371 & 0.43459 & 0.74955 & 0.74457 & 1.28668 & 1.28370 \\
\hline 7.0 & 0.47201 & 0.47293 & 0.77331 & 0.76737 & 1.33815 & 1.33496 \\
\hline 8.0 & 0.50387 & 0.50484 & 0.79187 & 0.78498 & 1.37775 & 1.37443 \\
\hline 9.0 & 0.53083 & 0.53182 & 0.80689 & 0.79906 & 1.40886 & 1.40547 \\
\hline 10.0 & 0.55396 & 0.55500 & 0.81940 & 0.81061 & 1.43370 & 1.43029 \\
\hline
\end{tabular}

Comparando-se os resultados da Tab. 3.4 com os das Tab. 3.1, 3.2 e 3.3 pode-se concluir que a diferença é de no máximo $1 \%$, o que mostra que neste caso, aplicando-se o carregamento em 10 ou 26 incrementos obtém-se praticamente os mesmos resultados.

A mesma viga foi analisada alterando-se o critério de convergência para $0,5 \%$ e para $1,0 \%$ e os resultados foram rigorosamente os mesmos, podendo-se concluir que pode-se trabalhar com uma tolerância de $1 \%$ em análises como esta (NLG).

\subsubsection{Exemplo 2: Pórtico Losangular sob Carga de Compressão}

Trata-se de um pórtico losangular sob carga de compressão, resolvido analiticamente por MATIASSON (1981). Devido à simetria da estrutura, apenas a metade do pórtico é resolvida. 
Foram utilizados os mesmos 26 incrementos de carga do Exemplo 1 e a mesma tolerância de $0,1 \%$.

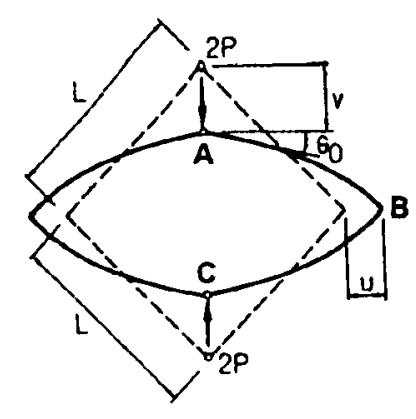

FIGURA 3.16 - Pórtico Losangular sob carga de compressão

As Tab. 3.5 e 3.6 mostram, respectivamente os valores dos deslocamentos adimensionais $\mathrm{u} / \ell$ do nó $\mathrm{B} \mathrm{e} \mathrm{v} / \ell$ do nó $\mathrm{A}$ (Fig. 3.16), quando as barras são discretizadas em 1, 2 ou 4 elementos, assim como os valores obtidos da solução analítica. As curvas carga $\mathrm{x}$ deslocamento são mostradas nas Fig. 3.17 e 3.18 .

TABELA 3.5 - Valores dos deslocamentos adimensionais $\mathrm{u} / \ell$ do nó $\mathrm{B}$ do pórtico da Fig. 3.16

\begin{tabular}{|c|c|c|c|c|}
\hline$\left(\mathbf{P L}^{2}\right) / \mathbf{E I}$ & $\begin{array}{c}\mathbf{1} \\
\text { Elemento }\end{array}$ & $\begin{array}{c}\mathbf{2} \\
\text { Elementos }\end{array}$ & $\begin{array}{c}\mathbf{4} \\
\text { Elementos }\end{array}$ & Analítico \\
\hline 0.0 & 0.00000 & 0.00000 & 0.00000 & 0.00000 \\
\hline 0.2 & 0.03378 & 0.03399 & 0.03406 & 0.03419 \\
\hline 0.4 & 0.06864 & 0.06914 & 0.06930 & 0.06958 \\
\hline 0.6 & 0.10395 & 0.10450 & 0.10472 & 0.10512 \\
\hline 0.8 & 0.13884 & 0.13887 & 0.13887 & 0.13932 \\
\hline 1.0 & 0.17223 & 0.17043 & 0.17010 & 0.17046 \\
\hline 1.2 & 0.20293 & 0.19796 & 0.19679 & 0.19689 \\
\hline 1.4 & 0.22980 & 0.22021 & 0.21778 & 0.21743 \\
\hline 1.6 & 0.25191 & 0.23659 & 0.23256 & 0.23161 \\
\hline 1.8 & 0.26868 & 0.24713 & 0.24128 & 0.23964 \\
\hline 2.0 & 0.27998 & 0.25235 & 0.24456 & 0.24224
\end{tabular}


TABELA 3.5 - Valores dos deslocamentos adimensionais $\mathrm{u} / \ell$ do nó $\mathrm{B}$ do pórtico da Fig. 3.16

\begin{tabular}{|c|c|c|c|c|}
\hline 2.5 & 0.28414 & 0.24865 & 0.23697 & 0.23108 \\
\hline 3.0 & 0.26555 & 0.22376 & 0.20997 & 0.20519 \\
\hline 3.5 & 0.23379 & 0.19526 & 0.17913 & 0.17335 \\
\hline 4.0 & 0.19574 & 0.16443 & 0.14673 & 0.14019 \\
\hline 4.5 & 0.15563 & 0.13372 & 0.11501 & 0.10788 \\
\hline 5.0 & 0.11582 & 0.10425 & 0.08496 & 0.07735 \\
\hline 5.5 & 0.07754 & 0.07649 & 0.05696 & 0.04893 \\
\hline 6.0 & 0.04136 & 0.05056 & 0.03104 & 0.02265 \\
\hline 6.5 & 0.00749 & 0.02643 & 0.00710 & -0.00158 \\
\hline 7.0 & -0.02404 & 0.00401 & -0.01492 & -0.02393 \\
\hline 7.5 & -0.05334 & -0.01684 & -0.03526 & -0.04455 \\
\hline 8.0 & -0.08540 & -0.03626 & -0.05407 & -0.06362 \\
\hline 8.5 & -0.10579 & -0.05438 & -0.07149 & -0.08129 \\
\hline 9.0 & -0.12925 & -0.07133 & -0.08767 & -0.09770 \\
\hline 9.5 & -0.15107 & -0.08723 & -0.10272 & -0.11298 \\
\hline 10.0 & -0.17140 & -0.10215 & -0.11676 & -0.12724 \\
\hline
\end{tabular}

TABELA 3.6 - Valores dos deslocamentos adimensionais $\mathrm{v} / \ell$ do nó $\mathrm{A}$ do pórtico da Fig. 3.16

\begin{tabular}{|c|c|c|c|c|}
\hline $\left.\mathbf{( P L}^{2}\right) / \mathbf{E I}$ & $\begin{array}{c}\mathbf{1} \\
\text { Elemento }\end{array}$ & $\begin{array}{c}\mathbf{2} \\
\text { Elementos }\end{array}$ & $\begin{array}{c}\mathbf{4} \\
\text { Elementos }\end{array}$ & Analítico \\
\hline 0.0 & 0.00000 & 0.00000 & 0.00000 & 0.00000 \\
\hline 0.2 & 0.03601 & 0.03627 & 0.03635 & 0.03630 \\
\hline 0.4 & 0.07725 & 0.07855 & 0.07898 & 0.07901 \\
\hline 0.6 & 0.12416 & 0.12729 & 0.12837 & 0.12862 \\
\hline 0.8 & 0.17695 & 0.18246 & 0.18447 & 0.18507 \\
\hline 1.0 & 0.23548 & 0.24338 & 0.24649 & 0.24754 \\
\hline 1.2 & 0.29919 & 0.30869 & 0.31288 & 0.31440 \\
\hline 1.4 & 0.36704 & 0.37647 & 0.38153 & 0.38343 \\
\hline 1.6 & 0.43763 & 0.44464 & 0.45018 & 0.45233 \\
\hline 1.8 & 0.50937 & 0.51131 & 0.51688 & 0.51910 \\
\hline 2.0 & 0.58072 & 0.57508 & 0.58023 & 0.58236 \\
\hline 2.5 & 0.74740 & 0.74014 & 0.70688 & 0.72091 \\
\hline 3.0 & 0.89139 & 0.83114 & 0.83089 & 0.83140 \\
\hline 3.5 & 1.01089 & 0.92305 & 0.91909 & 0.91852 \\
\hline 4.0 & 1.10855 & 0.99702 & 0.98934 & 0.98775
\end{tabular}


TABELA 3.6 - Valores dos deslocamentos adimensionais $\mathrm{v} / \ell$ do nó $\mathrm{A}$ do pórtico da Fig. 3.16

\begin{tabular}{|c|l|l|l|l|}
\hline 4.5 & 1.18822 & 1.05734 & 1.04610 & 1.04356 \\
\hline 5.0 & 1.25352 & 1.10731 & 1.09268 & 1.08927 \\
\hline 5.5 & 1.30747 & 1.14934 & 1.13151 & 1.12730 \\
\hline 6.0 & 1.35243 & 1.18522 & 1.16436 & 1.15940 \\
\hline 6.5 & 1.39025 & 1.21625 & 1.19251 & 1.18684 \\
\hline 7.0 & 1.42234 & 1.24342 & 1.21693 & 1.21058 \\
\hline 7.5 & 1.44497 & 1.26745 & 1.23834 & 1.23134 \\
\hline 8.0 & 1.47347 & 1.28890 & 1.25731 & 1.24966 \\
\hline 8.5 & 1.49403 & 1.30823 & 1.27425 & 1.26596 \\
\hline 9.0 & 1.51202 & 1.32576 & 1.28950 & 1.28056 \\
\hline 9.5 & 1.52785 & 1.34178 & 1.30333 & 1.29378 \\
\hline 10.0 & 1.54186 & 1.35649 & 1.31595 & 1.30578 \\
\hline
\end{tabular}

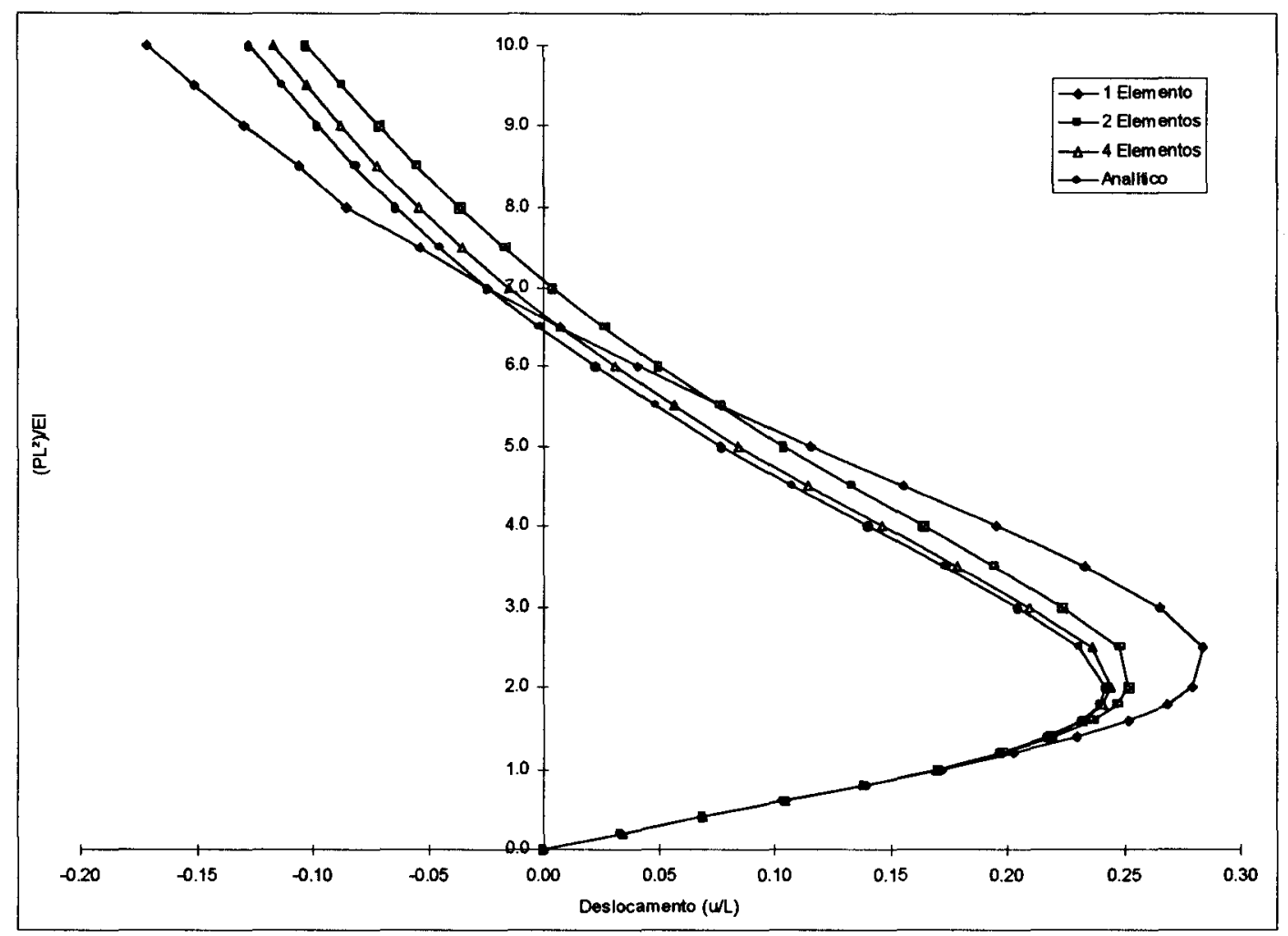

FIGURA 3.17 - Curva carga paramétrica $\mathrm{x}$ deslocamento adimensional $\mathrm{u} / \ell$ 


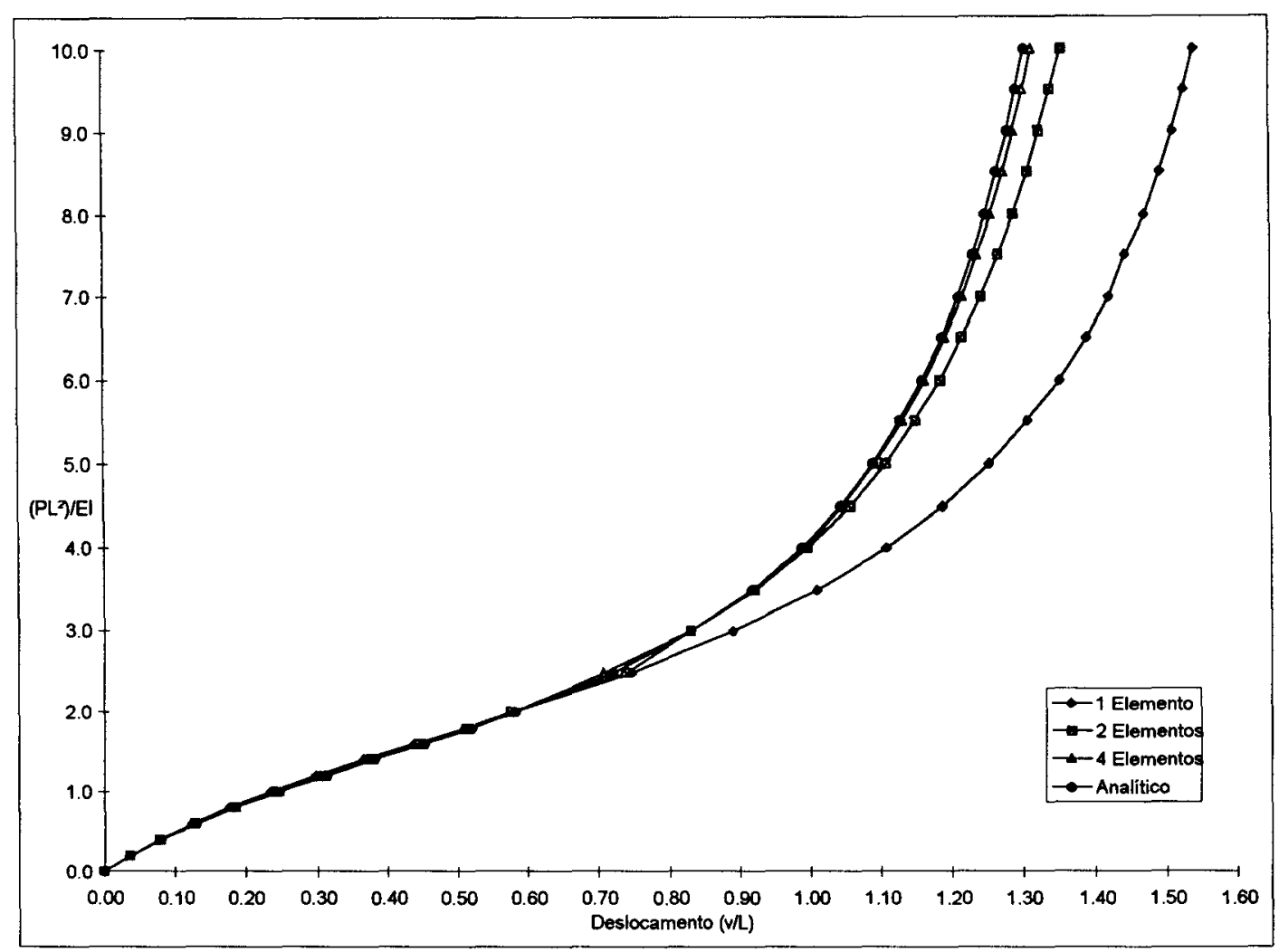

FIGURA 3.18 - Curva carga paramétrica $\mathrm{x}$ deslocamento adimensional $\mathrm{v} / \ell$

Da Tab. 3.6 observa-se que para deslocamentos verticais (v) de até $12 \%$ do comprimento $(\ell)$ da barra, ou seja, nível de carga igual a $0,6 \mathrm{PL}^{2} / \mathrm{EI}$, os resultados obtidos discretizando-se a barra com 1 elemento são praticamente iguais aos analíticos. Para deslocamentos verticais (v) até $58 \%$ do comprimento $(\ell)$ da barra, correspondente ao nível de carga igual a $2,0 \mathrm{PL}^{2} / \mathrm{EI}$, os resultados obtidos com 2 elementos em cada barra não diferem em mais de $1,3 \%$ dos resultados analíticos. Discretizando-se a barra em 4 elementos, o erro máximo obtido é da ordem de $1 \%$ para um deslocamento vertical de $1,3 \ell$.

A convergência ocorreu com no máximo 6 iterações.

\subsubsection{Exemplo 3: Pórtico Losangular sob Carga de Tração}

O mesmo pórtico do Exemplo 2 foi analisado sob carga de tração (Fig. 3.19), com o mesmo número de incrementos de carga. 


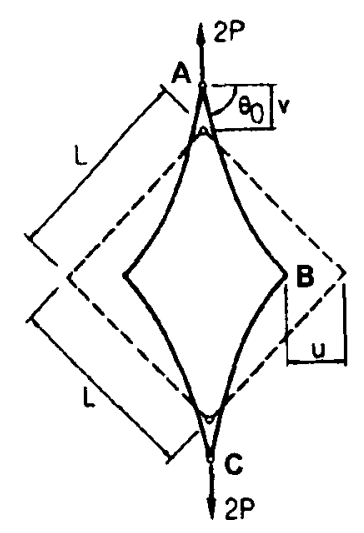

FIGURA 3.19 - Pórtico Losangular sob carga de tração

As Tab. 3.7 e 3.8 mostram, respectivamente os valores dos deslocamentos adimensionais $\mathrm{u} / \ell$ do nó $\mathrm{B}$ e $\mathrm{v} / \ell$ do nó $\mathrm{A}$ (Fig. 3.19), quando as barras são discretizadas em 1, 2 ou 4 elementos, assim como os valores obtidos da solução analítica por MATIASSON (1981). As curvas carga x deslocamento são mostradas nas Fig. 3.20 e 3.21 .

TABELA 3.7 - Valores dos deslocamentos adimensionais $\mathrm{u} / \ell$ do nó $\mathrm{B}$ do pórtico da Fig. 3.19

\begin{tabular}{|c|c|c|c|c|}
\hline$\left(\mathbf{P L}^{2}\right) / \mathbf{E I}$ & $\begin{array}{c}\mathbf{1} \\
\text { Elemento }\end{array}$ & $\begin{array}{c}\mathbf{2} \\
\text { Elementos }\end{array}$ & $\begin{array}{c}\mathbf{4} \\
\text { Elementos }\end{array}$ & Analítico \\
\hline 0.0 & 0.00000 & 0.00000 & 0.00000 & 0.00000 \\
\hline 0.2 & -0.03255 & -0.03233 & -0.03226 & -0.03233 \\
\hline 0.4 & -0.06329 & -0.06258 & -0.06237 & -0.06248 \\
\hline 0.6 & -0.09213 & -0.09068 & -0.09025 & -0.09036 \\
\hline 0.8 & -0.11910 & -0.11665 & -0.11595 & -0.11603 \\
\hline 1.0 & -0.14426 & -0.14060 & -0.13957 & -0.13960 \\
\hline 1.2 & -0.16769 & -0.16267 & -0.16129 & -0.16124 \\
\hline 1.4 & -0.18951 & -0.18303 & -0.18126 & -0.18112 \\
\hline 1.6 & -0.20984 & -0.20182 & -0.19964 & -0.19941 \\
\hline 1.8 & -0.22879 & -0.21919 & -0.21660 & -0.21627 \\
\hline 2.0 & -0.24648 & -0.23528 & -0.23227 & -0.23184 \\
\hline 2.5 & -0.28585 & -0.27071 & -0.26667 & -0.26594 \\
\hline 3.0 & -0.31933 & -0.30049 & -0.29546 & -0.29447 \\
\hline 3.5 & -0.34807 & -0.32586 & -0.31987 & -0.31865 \\
\hline 4.0 & -0.37297 & -0.34773 & -0.34089 & -0.33940 \\
\hline
\end{tabular}


TABELA 3.7 - Valores dos deslocamentos adimensionais $\mathrm{u} / \ell$ do nó $\mathrm{B}$ do pórtico da Fig. 3.19

\begin{tabular}{|c|c|c|c|c|}
\hline 4.5 & -0.39472 & -0.36667 & -0.35914 & -0.35742 \\
\hline 5.0 & -0.41385 & -0.38352 & -0.37515 & -0.37322 \\
\hline 5.5 & -0.43082 & -0.39838 & -0.38932 & -0.38720 \\
\hline $\mathbf{6 . 0}$ & -0.44595 & -0.41166 & -0.40196 & -0.39966 \\
\hline $\mathbf{6 . 5}$ & -0.45953 & -0.42361 & -0.41332 & -0.41086 \\
\hline 7.0 & -0.47178 & -0.43443 & -0.42359 & -0.42097 \\
\hline 7.5 & -0.48288 & -0.44289 & -0.43293 & -0.43016 \\
\hline $\mathbf{8 . 0}$ & -0.49299 & -0.45331 & -0.44147 & -0.43855 \\
\hline $\mathbf{8 . 5}$ & -0.50224 & -0.46159 & -0.44930 & -0.44625 \\
\hline 9.0 & -0.51072 & -0.46924 & -0.45653 & -0.45335 \\
\hline 9.5 & -0.51853 & -0.47633 & -0.46322 & -0.45992 \\
\hline 10.0 & -0.52574 & -0.48292 & -0.46943 & -0.46601 \\
\hline
\end{tabular}

TABELA 3.8 - Valores dos deslocamentos adimensionais $\mathrm{v} / \ell$ do nó $\mathrm{A}$ do pórtico da Fig. 3.19

\begin{tabular}{|c|c|c|c|c|}
\hline $\left.\mathbf{P L}^{2}\right) / \mathbf{E I}$ & $\begin{array}{c}\mathbf{1} \\
\text { Elemento }\end{array}$ & $\begin{array}{c}\mathbf{2} \\
\text { Elementos }\end{array}$ & $\begin{array}{c}\mathbf{4} \\
\text { Elementos }\end{array}$ & Analítico \\
\hline 0.0 & 0.00000 & 0.00000 & 0.00000 & 0.00000 \\
\hline 0.2 & 0.03102 & 0.03083 & 0.03077 & 0.03065 \\
\hline 0.4 & 0.05794 & 0.05707 & 0.05681 & 0.05648 \\
\hline 0.6 & 0.08135 & 0.07944 & 0.07888 & 0.07829 \\
\hline 0.8 & 0.10173 & 0.09857 & 0.09764 & 0.09677 \\
\hline 1.0 & 0.11953 & 0.11501 & 0.11370 & 0.11252 \\
\hline 1.2 & 0.13513 & 0.12921 & 0.12750 & 0.12601 \\
\hline 1.4 & 0.14886 & 0.14156 & 0.13946 & 0.13765 \\
\hline 1.6 & 0.16099 & 0.15235 & 0.14986 & 0.14774 \\
\hline 1.8 & 0.17174 & 0.16183 & 0.15899 & 0.15655 \\
\hline 2.0 & 0.18131 & 0.17022 & 0.16703 & 0.16429 \\
\hline 2.5 & 0.20097 & 0.18739 & 0.18344 & 0.17997 \\
\hline 3.0 & 0.21647 & 0.20058 & 0.19600 & 0.19183 \\
\hline 3.5 & 0.22816 & 0.21102 & 0.20589 & 0.20104 \\
\hline 4.0 & 0.23871 & 0.21948 & 0.21389 & 0.20839 \\
\hline 4.5 & 0.24570 & 0.22648 & 0.22049 & 0.21436 \\
\hline 5.0 & 0.25225 & 0.23239 & 0.22606 & 0.21931 \\
\hline 5.5 & 0.25778 & 0.23746 & 0.23083 & 0.22348 \\
\hline 6.0 & 0.26248 & 0.24187 & 0.23498 & 0.22703 \\
\hline
\end{tabular}


TABELA 3.8 - Valores dos deslocamentos adimensionais $\mathrm{v} / \ell$ do nó $\mathrm{A}$ do pórtico da Fig. 3.19

\begin{tabular}{|l|l|l|l|l|}
\hline 6.5 & 0.26655 & 0.24576 & 0.23864 & 0.23011 \\
\hline 7.0 & 0.27009 & 0.24923 & 0.24189 & 0.23279 \\
\hline 7.5 & 0.27320 & 0.25235 & 0.24483 & 0.23515 \\
\hline 8.0 & 0.27597 & 0.25518 & 0.24751 & 0.23726 \\
\hline 8.5 & 0.27845 & 0.25778 & 0.24996 & 0.23914 \\
\hline 9.0 & 0.28068 & 0.26018 & 0.25227 & 0.24084 \\
\hline 9.5 & 0.28272 & 0.26241 & 0.25433 & 0.24239 \\
\hline 10.0 & 0.28457 & 0.26448 & 0.25630 & 0.24380 \\
\hline
\end{tabular}

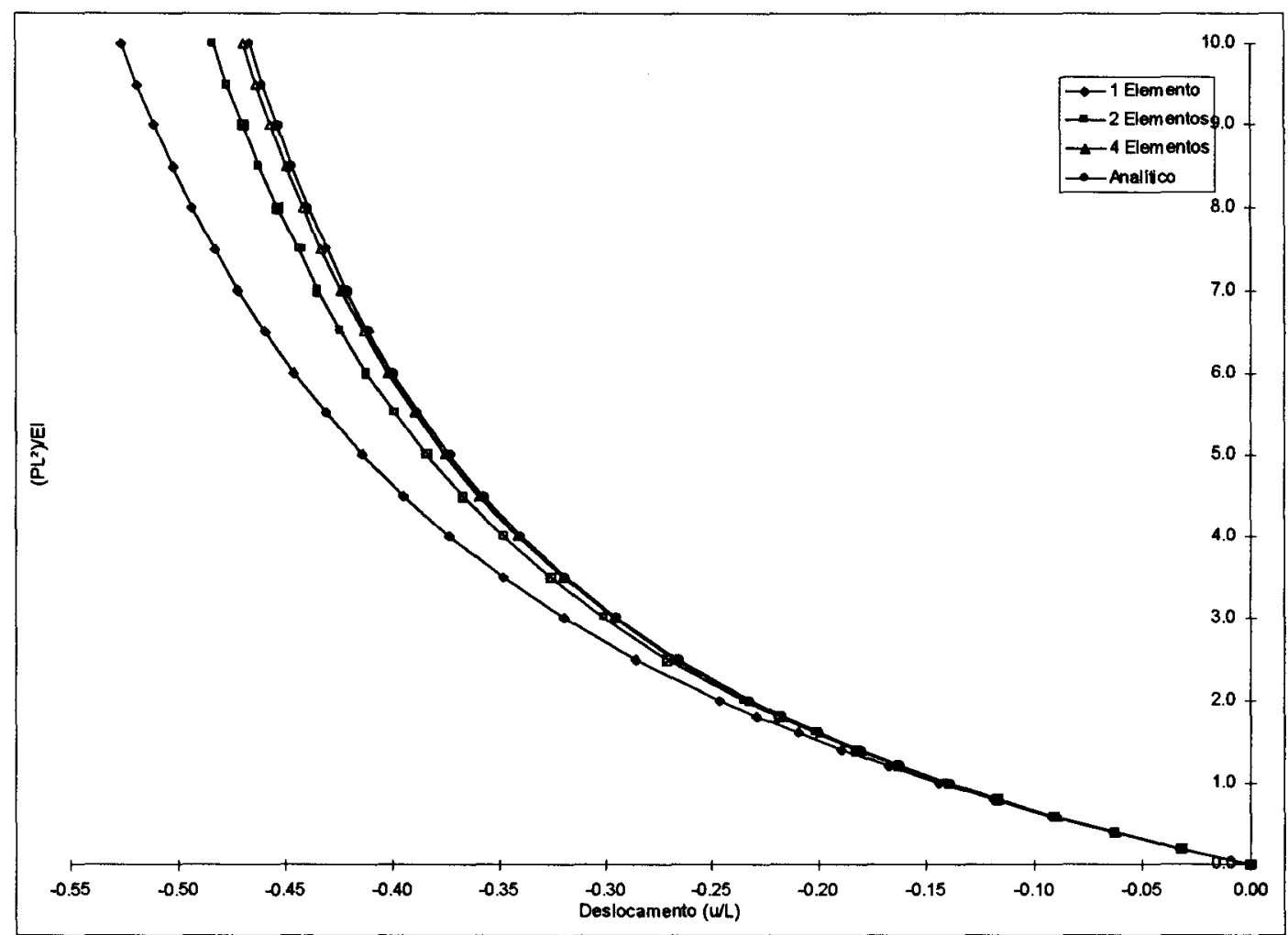

FIGURA 3.20 - Curva carga paramétrica $x$ deslocamento adimensional $\mathrm{u} / \ell$ 


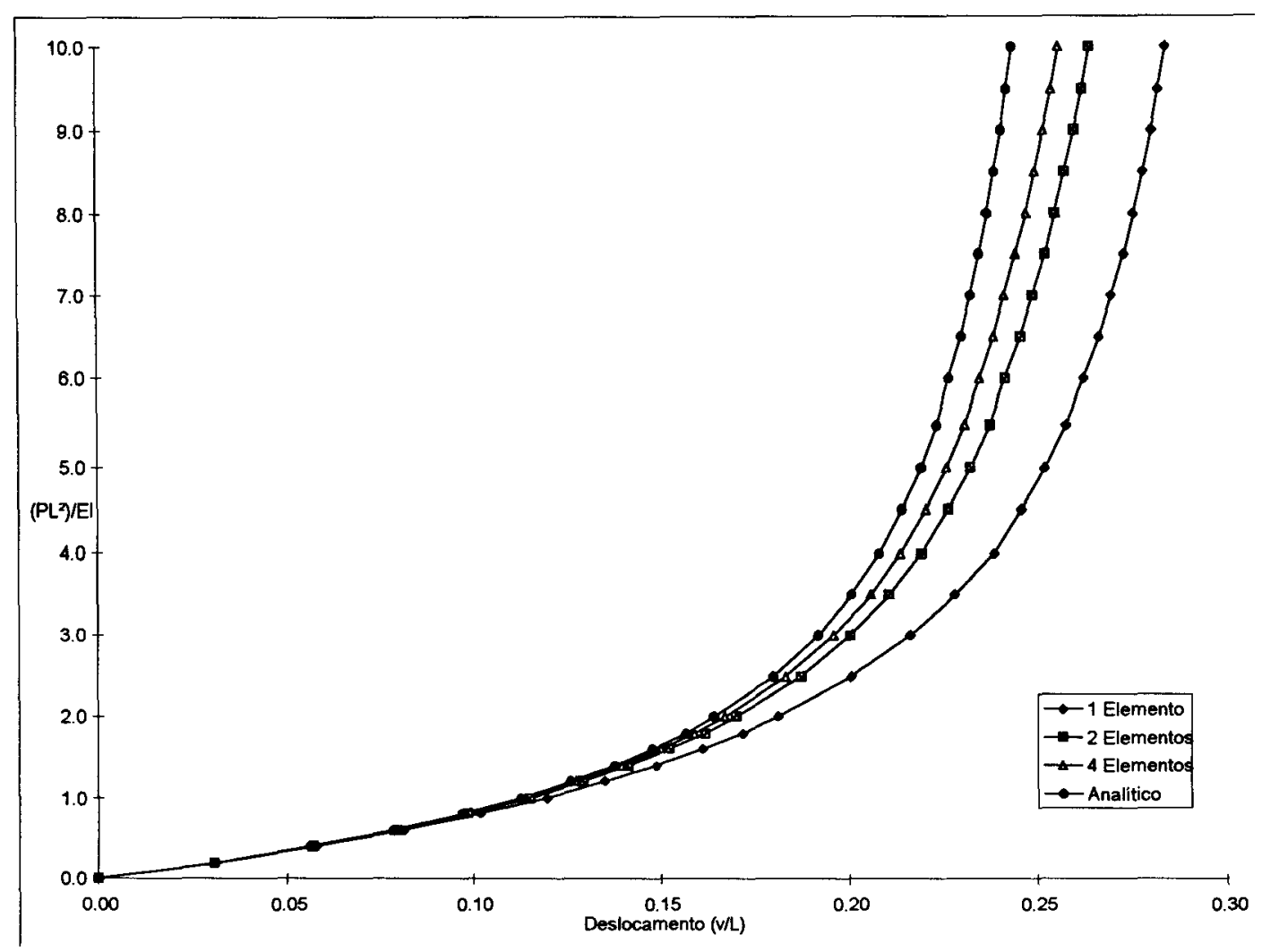

FIGURA 3.21 - Curva carga paramétrica $\mathrm{x}$ deslocamento adimensional $\mathrm{v} / \ell$

Da Tab. 3.7 observa-se que para deslocamentos horizontais (u) de até $11 \%$ do comprimento $(\ell)$ da barra, ou seja, nível de carga igual a $0,8 \mathrm{PL}^{2} / \mathrm{EI}$, os resultados obtidos discretizando-se a barra com 1 elemento são praticamente iguais aos analíticos. Para deslocamentos horizontais até $23 \%$ do comprimento $(\ell)$ da barra, correspondente ao nível de carga igual a 2,0PL $2 / \mathrm{EI}$, os resultados obtidos com 2 elementos em cada barra não diferem em mais de $1,5 \%$ dos resultados analíticos. Discretizando-se a barra em 4 elementos, o erro máximo obtido é da ordem de $0,7 \%$ para um deslocamento vertical de $0,47 \ell$.

A convergência ocorreu com no máximo 8 iterações, usando-se uma tolerância de $0,1 \%$. 


\subsubsection{Exemplo 4: Quadro sob Carga de Compressão}

Trata-se de um quadro (Fig. 3.22) sob carregamento de compressão, resolvido analiticamente por MATIASSON (1981). Na análise, é considerada a simetria do quadro em relação ao eixo vertical. As barras são discretizadas por 1,2 ou 4 elementos.

Foram utilizados 14 incrementos de carga.

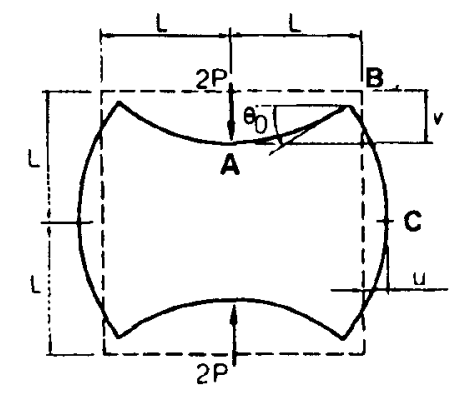

FIGURA 3.22 - Quadro sob carga de compressão

Nas Tab. 3.9, 3.10 e 3.11 são mostrados, respectivamente os valores dos deslocamentos adimensionais $\mathrm{u} / \ell$ do nó $\mathrm{C} \mathrm{e} \mathrm{v} / \ell$ do nó $\mathrm{A}$ e a rotação $\theta_{0}$ do nó $B$. As curvas carga $x$ deslocamento são mostradas nas Fig. 3.23, $3.24 \mathrm{e}$ 3.25 .

TABELA 3.9 - Valores dos deslocamentos adimensionais $\mathrm{u} / \ell$ do nó $\mathrm{C}$ do quadro da Fig. 3.22

\begin{tabular}{|c|c|c|c|c|}
\hline$\left(\mathbf{P L}^{2}\right) / \mathbf{E I}$ & $\begin{array}{c}\mathbf{1} \\
\text { Elemento }\end{array}$ & $\begin{array}{c}\mathbf{2} \\
\text { Elementos }\end{array}$ & $\begin{array}{c}\mathbf{4} \\
\text { Elementos }\end{array}$ & Analítico \\
\hline 0.0 & 0.00000 & 0.00000 & 0.00000 & 0.00000 \\
\hline 0.2 & 0.02503 & 0.02518 & 0.02518 & 0.02523 \\
\hline 0.4 & 0.05035 & 0.05075 & 0.05066 & 0.05084 \\
\hline 0.6 & 0.07592 & 0.07659 & 0.07630 & 0.07670 \\
\hline 0.8 & 0.10171 & 0.10254 & 0.10193 & 0.10264 \\
\hline
\end{tabular}


TABELA 3.9 - Valores dos deslocamentos adimensionais $\mathrm{u} / \ell$ do nó $\mathrm{C}$ do quadro da Fig. 3.22

\begin{tabular}{|l|l|l|l|l|}
\hline 1.0 & 0.12769 & 0.12844 & 0.12739 & 0.12850 \\
\hline 1.2 & 0.15384 & 0.15409 & 0.15248 & 0.15408 \\
\hline 1.4 & 0.18012 & 0.17929 & 0.17701 & 0.17914 \\
\hline 1.6 & 0.20648 & 0.20379 & 0.20074 & 0.20344 \\
\hline 1.8 & 0.23283 & 0.22733 & 0.22344 & 0.22668 \\
\hline 2.0 & 0.25904 & 0.24959 & 0.24483 & 0.24854 \\
\hline 2.5 & 0.32065 & 0.29733 & 0.29075 & 0.29483 \\
\hline 3.0 & 0.37396 & 0.33100 & 0.32333 & 0.32561 \\
\hline 3.5 & 0.41209 & 0.34891 & 0.34126 & 0.33921 \\
\hline 4.0 & 0.43042 & 0.35251 & 0.34589 & 0.33754 \\
\hline
\end{tabular}

TABELA 3.10 - Valores dos deslocamentos adimensionais $\mathrm{v} / \ell$ do nó $\mathrm{A}$ do quadro da Fig. 3.22

\begin{tabular}{|c|c|c|c|c|}
\hline $\left.\mathbf{P L}^{2}\right) / \mathbf{E I}$ & $\begin{array}{c}\mathbf{1} \\
\text { Elemento }\end{array}$ & $\begin{array}{c}\mathbf{2} \\
\text { Elementos }\end{array}$ & $\begin{array}{c}\mathbf{4} \\
\text { Elementos }\end{array}$ & Analítico \\
\hline 0.0 & 0.00000 & 0.00000 & 0.00000 & 0.00000 \\
\hline 0.2 & 0.04290 & 0.04307 & 0.04307 & 0.04293 \\
\hline 0.4 & 0.08768 & 0.08853 & 0.08849 & 0.08840 \\
\hline 0.6 & 0.13438 & 0.13640 & 0.13628 & 0.13645 \\
\hline 0.8 & 0.18303 & 0.18669 & 0.18638 & 0.18708 \\
\hline 1.0 & 0.23366 & 0.23937 & 0.23874 & 0.24025 \\
\hline 1.2 & 0.28635 & 0.29438 & 0.29324 & 0.29591 \\
\hline 1.4 & 0.34120 & 0.35164 & 0.34977 & 0.35397 \\
\hline 1.6 & 0.39830 & 0.41101 & 0.40816 & 0.41429 \\
\hline 1.8 & 0.45782 & 0.47231 & 0.46820 & 0.47668 \\
\hline 2.0 & 0.51988 & 0.53528 & 0.52964 & 0.54087 \\
\hline 2.5 & 0.68621 & 0.69775 & 0.68728 & 0.70665 \\
\hline 3.0 & 0.86751 & 0.86144 & 0.84516 & 0.87339 \\
\hline 3.5 & 1.05790 & 1.01851 & 0.99633 & 1.03230 \\
\hline 4.0 & 1.24472 & 1.16306 & 1.13568 & 1.17703 \\
\hline & & & & \\
\hline
\end{tabular}


TABELA 3.11 - Valores das rotações $\theta_{0}$ do nó B do quadro da Fig. 3.22

\begin{tabular}{|c|c|c|c|c|}
\hline $\left.\mathbf{( P L}^{2}\right) / \mathbf{E I}$ & $\begin{array}{c}\mathbf{1} \\
\text { Elemento }\end{array}$ & $\begin{array}{c}\mathbf{2} \\
\text { Elementos }\end{array}$ & $\begin{array}{c}\mathbf{4} \\
\text { Elementos }\end{array}$ & Analítico \\
\hline 0.0 & 0.00000 & 0.00000 & 0.00000 & 0.00000 \\
\hline 0.2 & 0.05125 & 0.05157 & 0.05157 & 0.05168 \\
\hline 0.4 & 0.10503 & 0.10627 & 0.10630 & 0.10674 \\
\hline 0.6 & 0.16138 & 0.16410 & 0.16420 & 0.16520 \\
\hline 0.8 & 0.22034 & 0.22496 & 0.22521 & 0.22699 \\
\hline 1.0 & 0.28192 & 0.28870 & 0.28923 & 0.29199 \\
\hline 1.2 & 0.34614 & 0.35513 & 0.35609 & 0.36000 \\
\hline 1.4 & 0.41303 & 0.42395 & 0.42554 & 0.43072 \\
\hline 1.6 & 0.48258 & 0.49481 & 0.49726 & 0.50378 \\
\hline 1.8 & 0.55476 & 0.56729 & 0.57084 & 0.57869 \\
\hline 2.0 & 0.62951 & 0.64089 & 0.64579 & 0.65486 \\
\hline 2.5 & 0.82539 & 0.82592 & 0.83506 & 0.84627 \\
\hline 3.0 & 1.03046 & 1.00491 & 1.01909 & 1.02992 \\
\hline 3.5 & 1.23557 & 1.16987 & 1.18904 & 1.19656 \\
\hline 4.0 & 1.43040 & 1.31640 & 1.33997 & 1.34181 \\
\hline
\end{tabular}

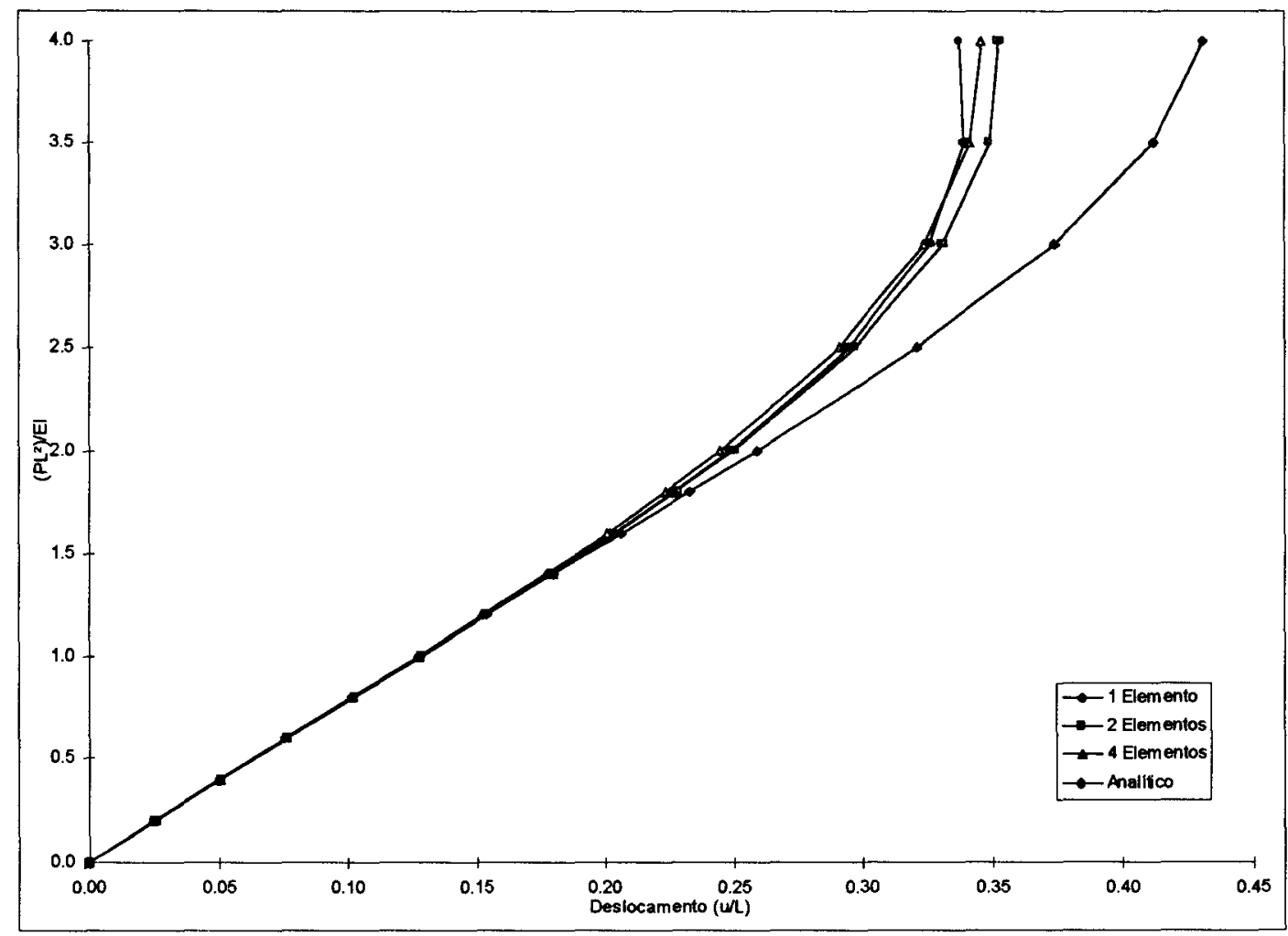

FIGURA 3.23 - Curva carga paramétrica $\mathrm{x}$ deslocamento adimensional $\mathrm{u} / \ell$ 


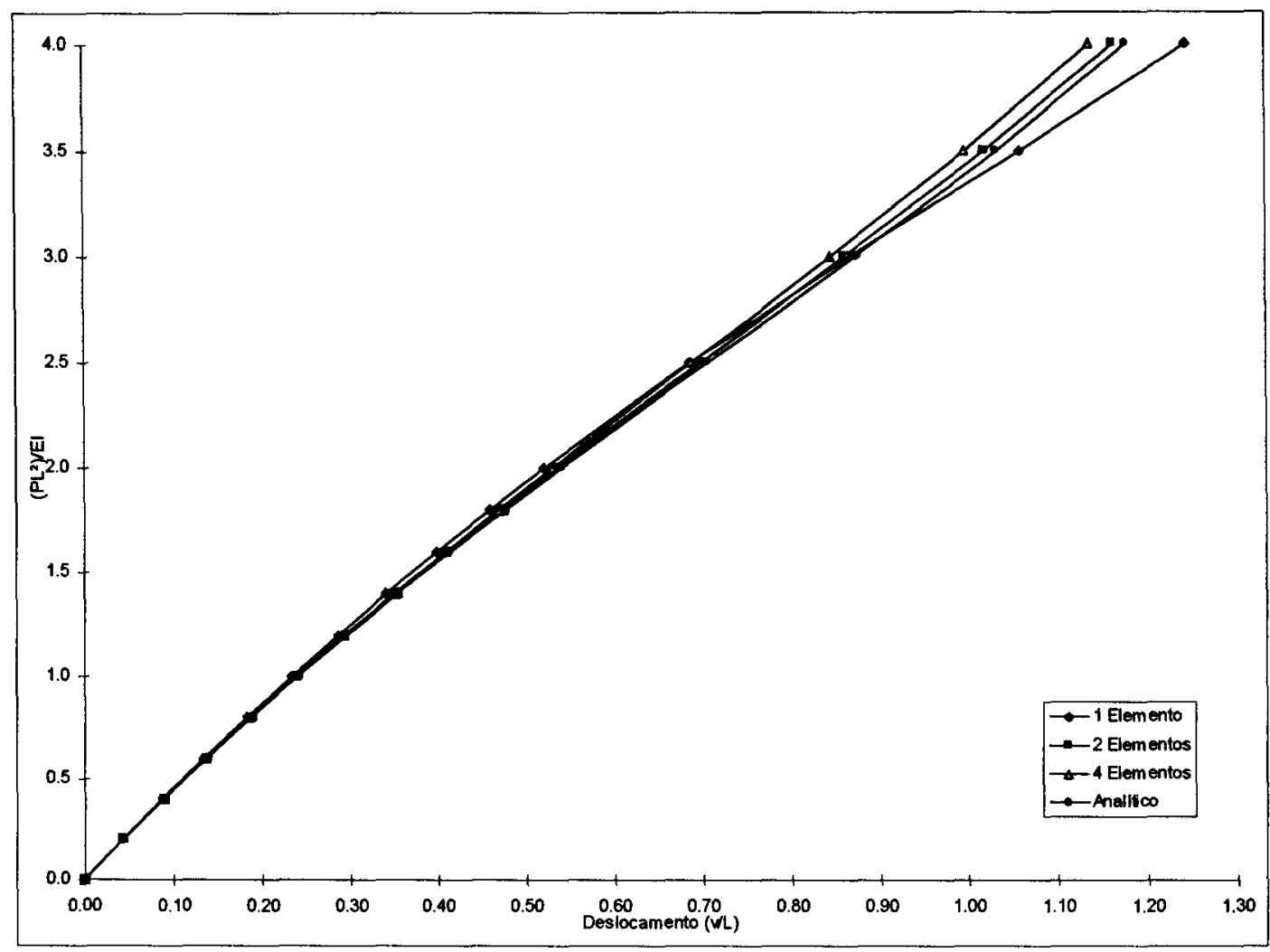

FIGURA 3.24 - Curva carga paramétrica $\mathrm{x}$ deslocamento adimensional $\mathrm{v} / \ell$

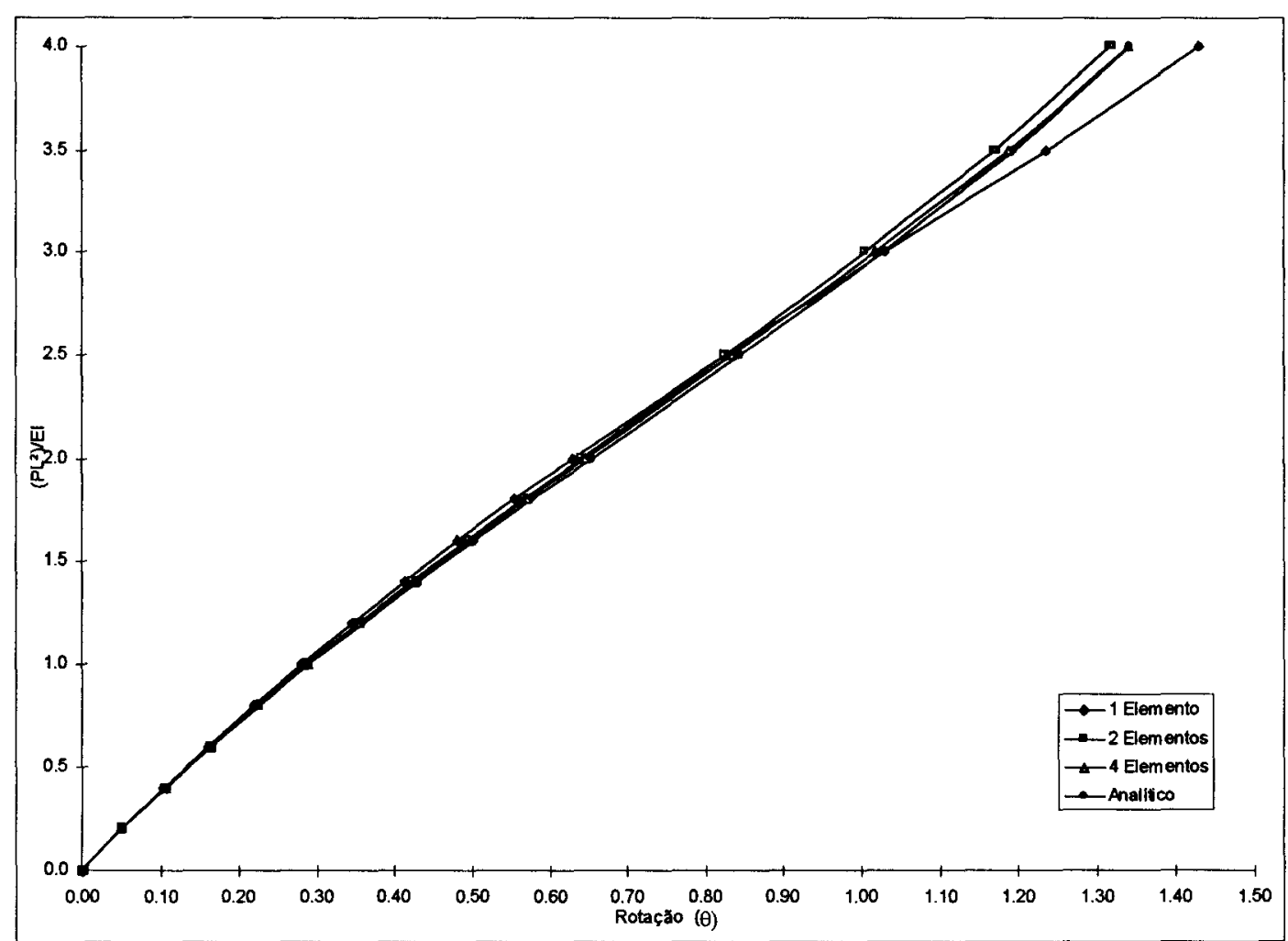

FIGURA 3.25 - Curva carga paramétrica $\mathrm{x}$ rotação $\theta_{0}$ 
Da Tab. 3.10 observa-se que para deslocamentos verticais (v) de até $13 \%$ do comprimento $(\ell)$ da barra, os valores obtidos discretizando-se a barra com 1 elemento diferem dos analíticos em menos de 1,5\%. Ao se discretizar as barras com 2 elementos os deslocamentos verticais de até $65 \%$ do comprimento $(\ell)$ da barra, o erro cometido não ultrapassa $2 \%$. Discretizandose cada barra com 4 elementos, o erro máximo não supera $1 \%$.

A convergência ocorreu com no máximo 6 iterações, usando-se uma tolerância de $0,1 \%$.

\subsubsection{Exemplo 5: Quadro sob Carga de Tração}

O mesmo quadro do Exemplo 4 foi analisado sob carga de tração (Fig. 3.26), com o mesmo número de incrementos de carga.

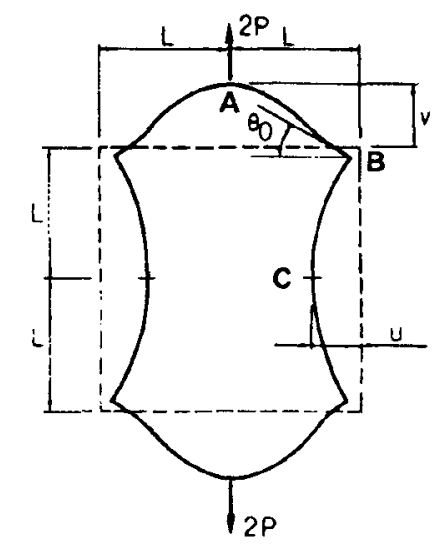

(a)

FIGURA 3.26 - Quadro sob carga de tração

As Tab. 3.12, 3.13 e 3.14 mostram, respectivamente os valores dos deslocamentos adimensionais $\mathrm{u} / \ell$ do nó $\mathrm{C}$ e $\mathrm{v} / \ell$ do nó $\mathrm{A}$ e a rotação $\theta_{0}$ do nó B. As curvas carga $x$ deslocamento são mostradas nas Fig. 3.27 e 3.28 e 3.29.

A convergência ocorreu com 6 iterações com uma tolerância de $0,1 \%$. 
TABELA 3.12 - Valores dos deslocamentos adimensionais $\mathrm{u} / \ell$ do nó $\mathrm{C}$ do quadro da Fig. 3.26

\begin{tabular}{|c|c|c|c|c|}
\hline $\left.\mathbf{P L}^{2}\right) / \mathbf{E I}$ & $\begin{array}{c}\mathbf{1} \\
\text { Elemento }\end{array}$ & $\begin{array}{c}\mathbf{2} \\
\text { Elementos }\end{array}$ & $\begin{array}{c}\mathbf{4} \\
\text { Elementos }\end{array}$ & Analítico \\
\hline 0.0 & 0.00000 & 0.00000 & 0.00000 & 0.00000 \\
\hline 0.2 & 0.02494 & 0.02478 & 0.02477 & 0.02473 \\
\hline 0.4 & 0.04946 & 0.04896 & 0.04901 & 0.04887 \\
\hline 0.6 & 0.07352 & 0.07245 & 0.07262 & 0.07233 \\
\hline 0.8 & 0.09708 & 0.09521 & 0.09553 & 0.09505 \\
\hline 1.0 & 0.12010 & 0.11721 & 0.11769 & 0.11699 \\
\hline 1.2 & 0.14254 & 0.13841 & 0.13906 & 0.13813 \\
\hline 1.4 & 0.16439 & 0.15881 & 0.15962 & 0.15845 \\
\hline 1.6 & 0.18562 & 0.17840 & 0.17938 & 0.17795 \\
\hline 1.8 & 0.20621 & 0.19721 & 0.19833 & 0.19664 \\
\hline 2.0 & 0.22617 & 0.21523 & 0.21648 & 0.21453 \\
\hline 2.5 & 0.27332 & 0.25713 & 0.25853 & 0.25595 \\
\hline 3.0 & 0.31646 & 0.29470 & 0.29612 & 0.29298 \\
\hline 3.5 & 0.35583 & 0.32844 & 0.32974 & 0.32611 \\
\hline 4.0 & 0.39169 & 0.35881 & 0.35985 & 0.35581 \\
\hline
\end{tabular}

TABELA 3.13 - Valores dos deslocamentos adimensionais $\mathrm{v} / \ell$ do nó $\mathrm{A}$ do quadro da Fig. 3.26

\begin{tabular}{|c|c|c|c|c|}
\hline $\left.\mathbf{P L}^{2}\right) / \mathbf{E I}$ & $\begin{array}{c}\mathbf{1} \\
\text { Elemento }\end{array}$ & $\begin{array}{c}\mathbf{2} \\
\text { Elementos }\end{array}$ & $\begin{array}{c}\mathbf{4} \\
\text { Elementos }\end{array}$ & Analítico \\
\hline 0.0 & 0.00000 & 0.00000 & 0.00000 & 0.00000 \\
\hline 0.2 & 0.04082 & 0.04067 & 0.04067 & 0.04043 \\
\hline 0.4 & 0.07978 & 0.07907 & 0.07905 & 0.07843 \\
\hline 0.6 & 0.11692 & 0.11529 & 0.11521 & 0.11410 \\
\hline 0.8 & 0.15230 & 0.14942 & 0.14922 & 0.14755 \\
\hline 1.0 & 0.18594 & 0.18157 & 0.18121 & 0.17889 \\
\hline 1.2 & 0.21792 & 0.21186 & 0.21126 & 0.20825 \\
\hline 1.4 & 0.24828 & 0.24039 & 0.23949 & 0.23575 \\
\hline 1.6 & 0.27710 & 0.26730 & 0.26603 & 0.26152 \\
\hline 1.8 & 0.30445 & 0.29268 & 0.29099 & 0.28567 \\
\hline 2.0 & 0.33039 & 0.31664 & 0.31446 & 0.30833 \\
\hline 2.5 & 0.38934 & 0.37091 & 0.36734 & 0.35917 \\
\hline 3.0 & 0.44113 & 0.41836 & 0.41314 & 0.40287 \\
\hline 3.5 & 0.48674 & 0.46013 & 0.45310 & 0.44073 \\
\hline 4.0 & 0.52710 & 0.49720 & 0.48822 & 0.47375 \\
\hline
\end{tabular}


TABELA 3.14 - Valores das rotações $\theta_{0}$ do nó $B$ do quadro da Fig. 3.26

\begin{tabular}{|c|c|c|c|c|}
\hline$\left(\mathbf{P L}^{2}\right) /$ EI & $\begin{array}{c}\mathbf{1} \\
\text { Elemento }\end{array}$ & $\begin{array}{c}\mathbf{2} \\
\text { Elementos }\end{array}$ & $\begin{array}{c}\mathbf{4} \\
\text { Elementos }\end{array}$ & Analítico \\
\hline 0.0 & 0.00000 & 0.00000 & 0.00000 & 0.00000 \\
\hline 0.2 & 0.04875 & 0.04847 & 0.04844 & 0.04835 \\
\hline 0.4 & 0.09505 & 0.09385 & 0.09383 & 0.09346 \\
\hline 0.6 & 0.13897 & 0.13626 & 0.13626 & 0.13548 \\
\hline 0.8 & 0.18058 & 0.17585 & 0.17585 & 0.17454 \\
\hline 1.0 & 0.21997 & 0.21276 & 0.21276 & 0.21082 \\
\hline 1.2 & 0.25723 & 0.24172 & 0.24712 & 0.24449 \\
\hline 1.4 & 0.29245 & 0.27910 & 0.27910 & 0.27573 \\
\hline 1.6 & 0.32574 & 0.30886 & 0.30886 & 0.30471 \\
\hline 1.8 & 0.35719 & 0.33656 & 0.33656 & 0.33161 \\
\hline 2.0 & 0.38691 & 0.36234 & 0.36234 & 0.35658 \\
\hline 2.5 & 0.45417 & 0.41936 & 0.41936 & 0.41156 \\
\hline 3.0 & 0.51264 & 0.46734 & 0.46734 & 0.45752 \\
\hline 3.5 & 0.56363 & 0.50794 & 0.50794 & 0.49618 \\
\hline 4.0 & 0.60829 & 0.54253 & 0.54253 & 0.52892 \\
\hline
\end{tabular}

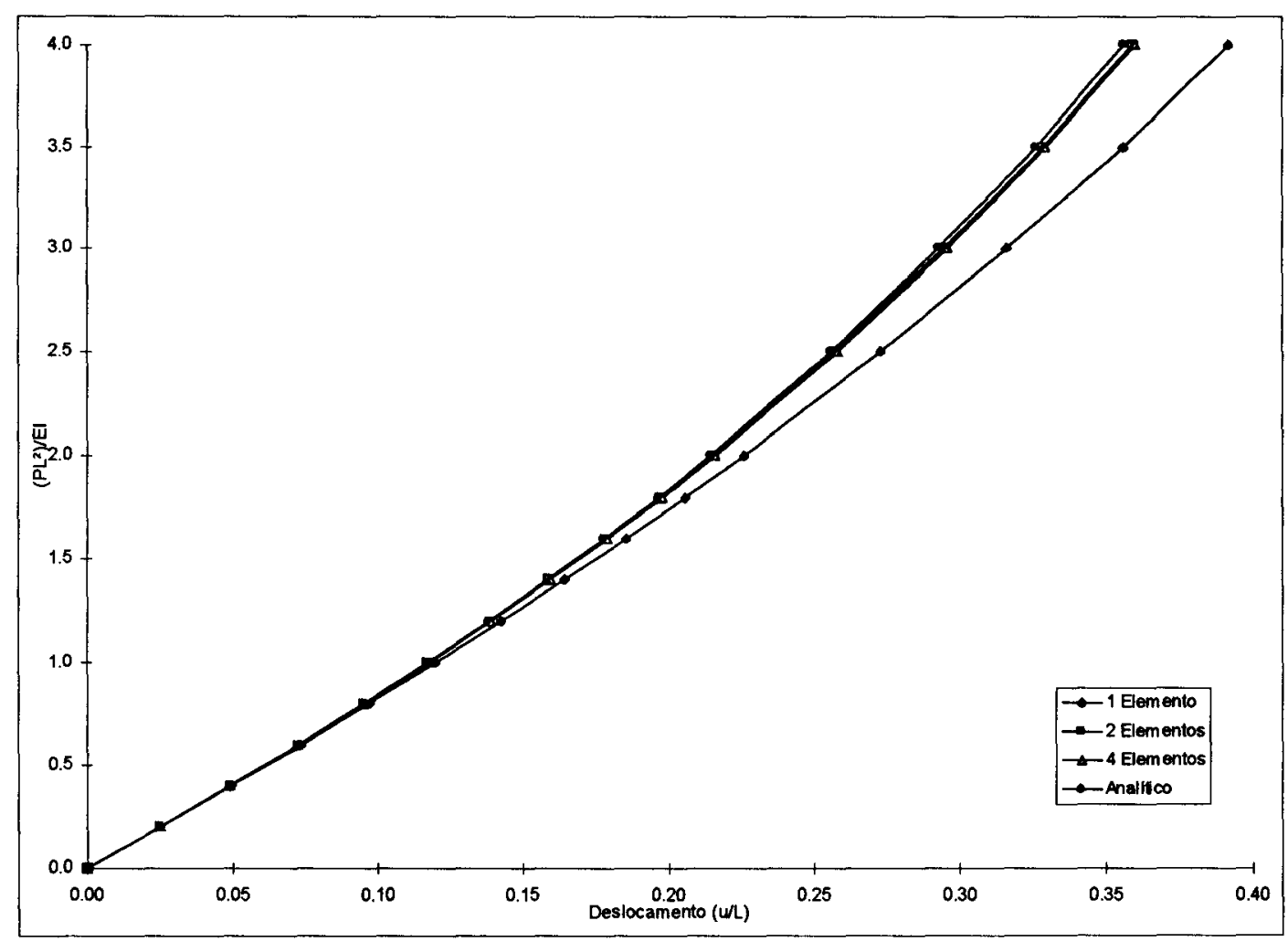

FIGURA 3.27 - Curva carga paramétrica $\mathrm{x}$ deslocamento adimensional $\mathrm{u} / \ell$ 


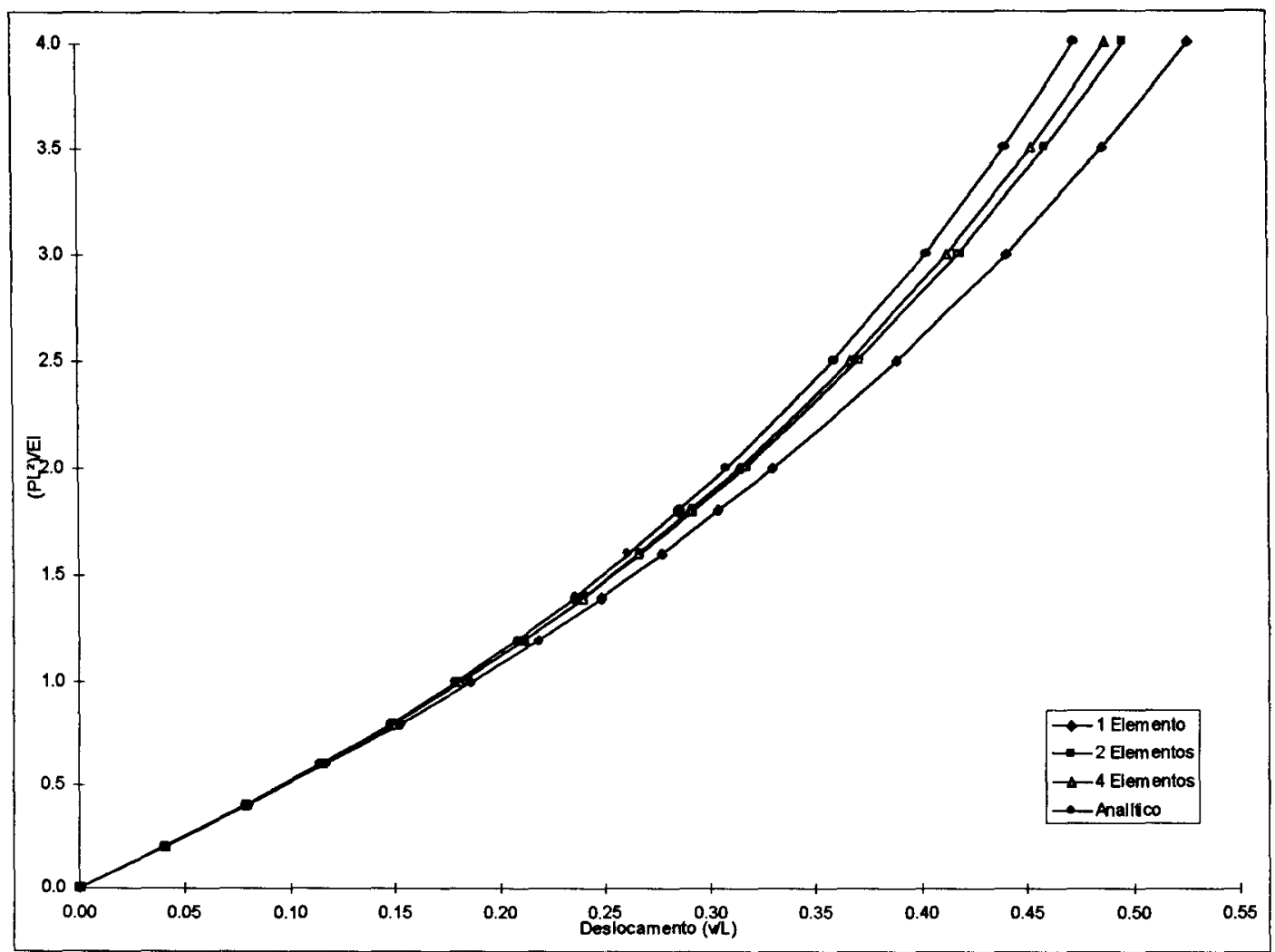

FIGURA 3.28 - Curva carga paramétrica $\mathrm{x}$ deslocamento adimensional $\mathrm{v} / \ell$

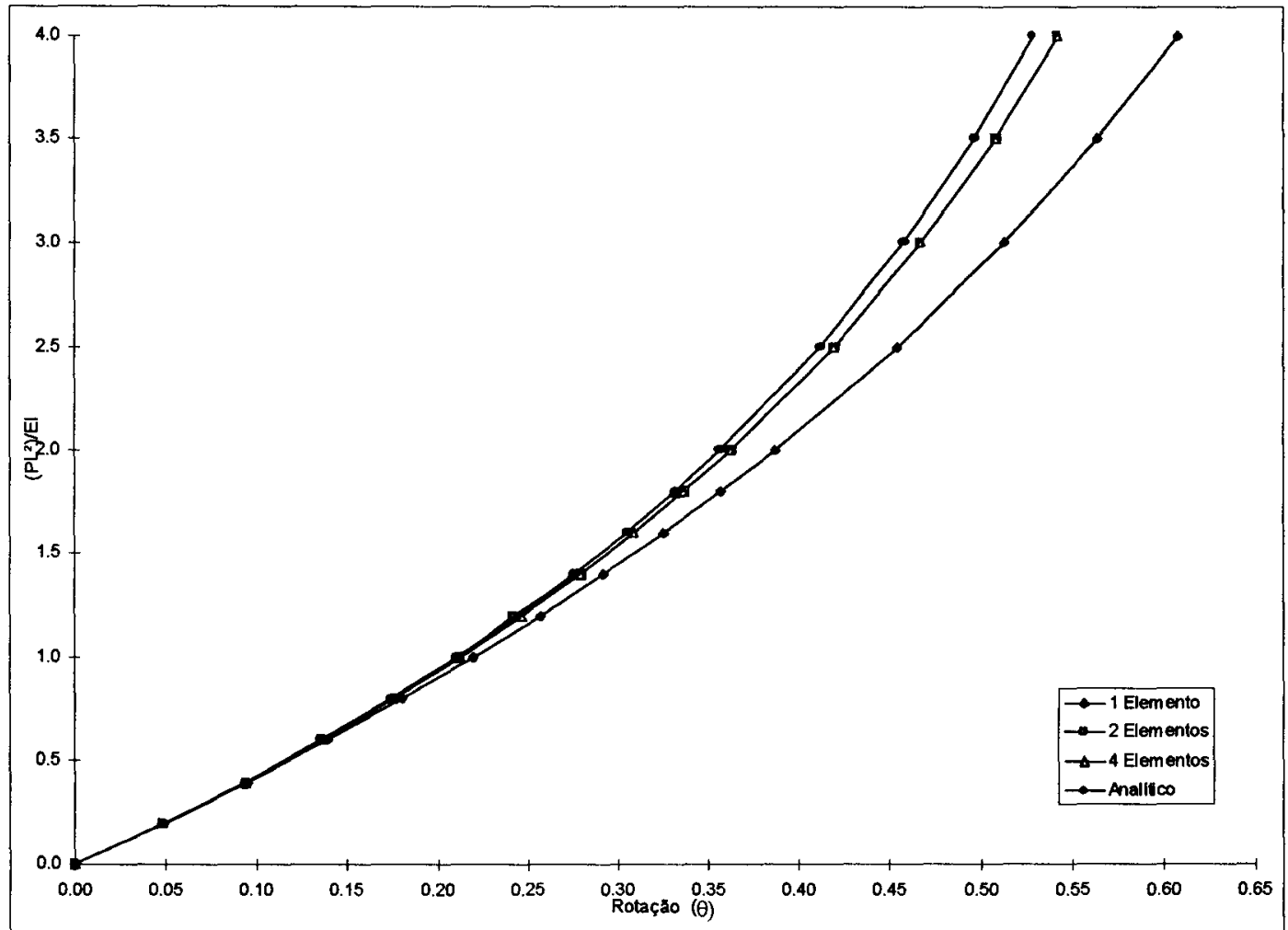

FIGURA 3.29 - Curva carga paramétrica $\times$ rotação $\theta_{0}$ 
Da Tab. 3.13 observa-se que:

- discretizando-se as barras com 1 elemento, os valores obtidos para v têm uma boa aproximação com os analíticos até o nível de carga igual a $0,6 \mathrm{PL}{ }^{2} / \mathrm{EI}$, correspondendo a $\mathrm{v} \cong 0,11 \ell$;

- discretizando-se as barras com 2 elementos, os valores obtidos para $v$ têm uma boa aproximação com os analíticos até o nível de carga igual a $1,4 \mathrm{PL}^{2} / \mathrm{EI}$, correspondendo a $\mathrm{v} \cong 0,24 \ell$;

- discretizando-se as barras com 4 elementos o erro máximo nos valores dos deslocamentos v é de $2,5 \%$. 


\section{APLICAÇÃO DA FORMULAÇÃO CONSISTENTE À ANÁLISE DE PÓRTICOS PLANOS DE CONCRETO ARMADO}

\subsection{Introdução}

Um grande número de modelos físicos e matemáticos têm sido usados para pesquisar o comportamento das estruturas de concreto armado. Em geral, os modelos físicos são desenvolvidos para verificar modelos matemáticos. Os modelos matemáticos partem de formulações mais simples apropriadas para cálculos manuais até envolver modelos mais complexos que requerem o uso de computadores.

Segundo MARTINS (1995), ao se analisar uma estrutura de concreto armado deve-se ter bem claro os objetivos a alcançar de modo que a escolha do modelo e dos métodos de análise sejam compatíveis com estes objetivos. Neste capítulo admite-se que foi feita a op̧̧ão pela análise não-linear da estrutura de concreto armado.

Numa análise não-linear deve-se distinguir se o objetivo é uma verificação em Estado Limite Último normalizado ou uma pesquisa da capacidade portante real da estrutura.

$\mathrm{Na}$ pesquisa da capacidade portante não estão, em princípio, incluídos critérios normativos de segurança, procura-se apenas qual a resposta dada pela estrutura a uma dada disposição de cargas crescentes, consideradas as características reais dos materiais e das cargas. Em geral os materiais são 
definidos pelos valores médios de suas características mecânicas, enquanto que as cargas pelos seus valores prováveis.

No caso da verificação do Estado Limite Último, é fundamental o conceito de critérios normalizados de segurança. Como exemplo o critério adotado pela Norma Brasileira e pelo Código Modelo (CEB/FIP 90) define os materiais pelos valores de cálculo de suas resistências, enquanto que a resposta da análise fornecerá o coeficiente de segurança que a estrutura apresenta em relação aos valores característicos das cargas aplicadas.

\subsection{Revisão Bibliográfica}

O estudo do comportamento estrutural não-linear das estruturas de concreto armado tem atraído muito a atenção dos pesquisadores. Pode-se observar nas duas últimas décadas uma grande quantidade de trabalhos publicados a respeito do mesmo. Alguns destes trabalhos são citados a seguir:

AAS-JAKOBSEN \& GRENACHER (1974) propuseram um método de análise não-linear de pórticos em concreto armado via MEF utilizando matriz de rigidez secante e usando uma curva real tensão $\mathrm{x}$ deformação para $\mathrm{o}$ concreto.

TELES (1976) estudou a não-linearidade física e geométrica de pórticos planos de concreto armado no âmbito dos pequenos deslocamentos utilizando as funções de estabilidade para obtenção da matriz de rigidez NLG; o comportamento não-linear do concreto armado (NLF) é considerado através de módulos de elasticidade secantes obtidos de diagramas tensão-deformação específicos para o aço e o concreto.

MARTINS (1979) estende este estudo a pórticos espaciais.

No campo da não-linearidade fisica de estruturas de concreto armado, houve a partir da década de 80 um incontável número de trabalhos publicados; a seguir serão citados os principais consultados pelo autor. 
GREUNEN \& SCORDELIS (1983) mostram um eficiente procedimento numérico para análise não-linear de estruturas de concreto armado e protendido incluindo efeitos reológicos e de temperatura, baseado no MEF utilizando formulação Lagrangiana atualizada para levar em conta os efeitos da mudança de geometria.

KAYAL (1984) mostra um elemento finito para análise não-linear com cinco graus de liberdade (duas rotações, duas deflexões nas extremidades, e uma deflexão no meio do vão). Integração analítica leva a uma solução de forma fechada para avaliar a contribuição do concreto.

MARI et al (1984) estende o estudo à análise de pórticos espaciais, utilizando o modelo material sugerido por HOGNESTAD (1951), incluindo fissuração, resistência à tração, creep e cargas reversas.

PROENÇA (1989) apresenta um estudo sobre modelos matemáticos do comportamento não-linear físico do concreto.

EL-METWALLY \& CHEN (1989), JORDAN \& KREGER (1991) incluíram a consideração da flexibilidade dos nós na análise não-linear de pórticos planos e espaciais.

ALWIS (1990) apresenta uma curva trilinear momento-curvatura para barras de concreto armado onde os comportamentos não fissurado, fissurado $e$ plástico são representados. Apenas três variáveis (módulo de elasticidade, resistência à tração e resistência à compressão) são usadas para definir as propriedades do concreto.

CILONI (1993) a partir do trabalho de CAUVIN (1979) desenvolveu programa de análise não-linear de concreto armado introduzindo a contribuição do concreto tracionado intacto entre as fissuras como nos modelos do CEB, e utilizou um campo de deformação mais completo que o de CAUVIN. A não-linearidade do concreto é baseada em relações momentocurvatura, utilizando a formulação lagrangiana com coordenadas cartesianas para obtenção da matriz de rigidez tangente. 
RASHEED \& DINNO (1993) mostram um eficiente algoritmo de análise incremental-iterativa de seções de concreto armado usando a rigidez secante. Um esquema de solução não-linear é utilizado para obtenção de forma fechada das propriedades não-lineares da seção ao invés de se ter um número finito de fatias integradas numericamente, aumentando assim a precisão dos resultados.

IZZUDDIN et al (1994) apresentam uma nova formulação para modelagem de resposta não-linear elástica de barras de concreto armado, adequada para uso da eficiente técnica adaptativa na análise de grandes deslocamentos ineslásticos de pórticos de concreto armado. O elemento finito elástico é obtido no sistema de coordenadas euleriano com quatro graus de liberdade naturais. A função deslocamento transversal é de quarto grau e é adotado o critério da força axial constante. A análise não-linear inicia usando um elemento por barra. Durante a análise várias partes da estrutura são checadas quanto ao aparecimento de inelasticidades, e elementos baseados na discretização da seção em fatias são inseridas nas zonas inelásticas. KARAYANNIS et al (1994) ampliam a aplicação desta técnica adaptativa na análise inelástica, apresentando um elemento inelástico com função interpoladora de terceiro grau baseado no processo das fatias, que conjugado com o elemento elástico completa a metodologia proposta. $\mathrm{O}$ aparecimento de zonas inelásticas provoca um refinamento automático da malha de elementos finitos. Esta técnica adaptativa leva a uma considerável economia computacional, uma vez que elementos baseados no processo das fatias são inseridos apenas quando e onde necessário, durante a análise.

SOLER (1995) apresenta uma teoria de barras retas no espaço que pode ser utilizada na análise não-linear de pórticos espaciais de concreto armado. As equações de equilíbrio são formuladas variacionalmente e as rotações no espaço são tratadas de forma Lagrangiana, por meio da fórmula de EulerRodrigues. A teoria não-linear utilizada é geometricamente exata. 


\subsection{Elemento Prismático de Concreto Armado}

Conforme observado por CILONI (1993), "inúmeros pesquisadores se dedicaram a cobrir diversos aspectos do comportamento das estruturas de concreto armado. MARI (1984) classifica estes aspectos em três grupos principais:

I. modelagem das propriedades dos materiais (equações constitutivas, estados de tensão, comportamento ao longo do tempo, etc. ...)

II. estudos a nível "microscópico" (degradação da aderência, transferência de esforços de cisalhamento, colaboração do concreto tracionado intacto entre fissuras - "tension stiffening", etc. ...)

III. estudo a nível "macroscópico", em que se tenta modelar o comportamento global da estrutura ao invés de priorizar efeitos locais"

Em CILONI (1993) são detalhados os aspectos relativos aos grupos I e II e uma vasta bibliografia é citada. Os objetivos deste trabalho se enquadram no grupo III, ou seja, dentre as inúmeras relações para descrever o comportamento tensão-deformação para o concreto propostas na literatura, será adotada aquela proposta por HOGNESTAD (1951) que, com algumas simplificações, é recomendada pela NBR-6118. A partir desta relação e com algumas hipóteses de comportamento a nível microscópico, usando a formulação consistente desenvolvida neste trabalho, chega-se ao comportamento não-linear dos pórticos planos de concreto armado. 


\subsubsection{Utilização do Processo de Fatias para a Discretização da Seção do Elemento de Concreto Armado}

O processo das fatias consiste em dividir a seção tranversal em fatias de tal maneira que se possa obter a resposta desta seção a partir das respostas das fatias individuais para as quais as relações tensão-deformação realísticas são aplicadas. A utilização deste processo permite o uso de relações constitutivas uniaxiais entre tensão e deformação para o concreto e o aço. Através deste processo as integrações na seção para obtenção das propriedades gemométricas (área, momento estático e momento de inércia) e para cálculo de esforços solicitantes (força normal e momento fletor) se transformam numa somatória discreta.

A decomposição típica de uma seção transversal de um elemento de concreto armado é mostrada na Fig. 4.1.

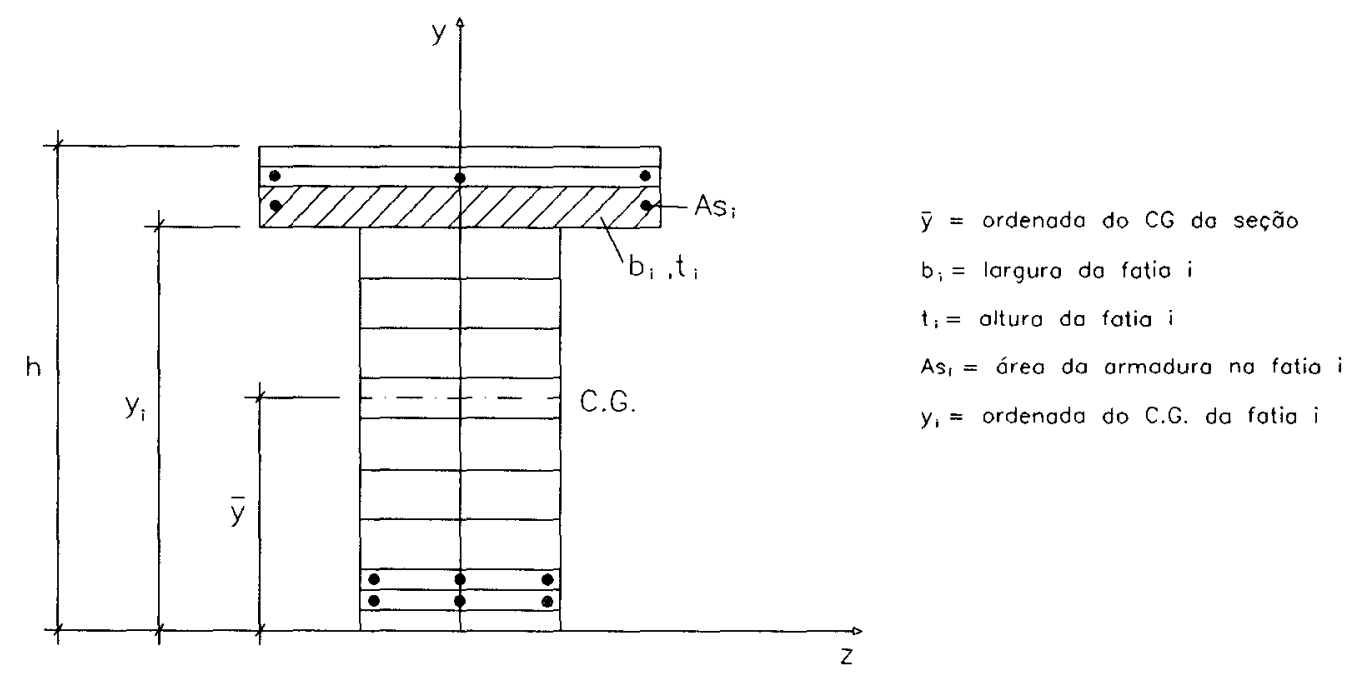

FIGURA 4.1 - Seção tranversal dividida em fatias

Admite-se que as tensões são constantes na fatia.

Os esforços solicitantes $\mathrm{N}$ e M na seção tranversal serão dados por:

$$
N=\int_{A_{r}} \sigma d A_{r}=\sum_{i=1}^{m}\left(f_{c i} b_{i} t_{i}+f_{s i} A_{s i}\right)
$$




$$
M=-\int_{A_{r}} \sigma y_{r} d A_{r}=\sum_{i=1}^{m}\left(-f_{c i} b_{i} t_{i}-f_{s i} A_{s i}\right)\left(y_{i}-\bar{y}\right)
$$

onde

$$
\begin{aligned}
& \mathrm{m}=\text { número de fatias da seção } \\
& \mathrm{b}_{\mathrm{i}}=\text { largura da fatia } \mathrm{i} \\
& \mathrm{t}_{\mathrm{i}}=\text { altura da fatia } \mathrm{i} \\
& \mathrm{y}_{\mathrm{i}}=\text { ordenada do } \mathrm{CG} \text { da fatia } \mathrm{i} \\
& \overline{\mathrm{y}}=\text { ordenada do } \mathrm{CG} \text { da seção tranversal } \\
& \mathrm{A}_{\mathrm{si}}=\text { área total da armadura na fatia } \mathrm{i} \\
& \mathrm{f}_{\mathrm{ci}}=\text { tensão no concreto na fatia } \mathrm{i} \\
& \dot{f}_{\mathrm{si}}=\text { tensão na armadura na fatia } \mathrm{i}
\end{aligned}
$$

As constantes $C_{1}, C_{2}$ e $C_{3}$ dadas pelas equações 3.213 a 3.215 serão calculadas como:

$$
\begin{aligned}
& C_{1}=\int_{A_{r}} D d A_{r}=\sum_{i=1}^{m}\left(D_{c i} b_{i} t_{i}+D_{s i} A_{s i}\right) \\
& C_{2}=\int_{A_{r}} D y_{r}^{2} d A_{r}=\sum_{i=1}^{m}\left[D_{c i}\left(y_{i}-\bar{y}\right)^{2} b_{i} t_{i}+D_{s i}\left(y_{i}-\bar{y}\right)^{2} A_{s i}\right] \\
& C_{3}=\int_{A_{r}} D y_{r} d A_{r}=\sum_{i=1}^{m}\left[D_{c_{i}}\left(y_{i}-\bar{y}\right) b_{i} t_{i}+D_{s i}\left(y_{i}-\bar{y}\right) A_{s i}\right]
\end{aligned}
$$

onde

$\mathrm{D}_{\mathrm{ci}}=$ módulo tangente do concreto na fatia $i$ obtido da relação constitutiva adotada para o concreto 
$\mathrm{D}_{\mathrm{si}}=$ módulo tangente do aço na fatia $i$ obtido da relação constitutiva adotada para 0 aço

\subsubsection{Equações Constitutivas para o Concreto}

Várias relações para descrever o comportamento tensão-deformação para o concreto em compressão uniaxial são apresentadas na literatura. Dentre estas relações, uma delas, originalmente proposta por SARGIN (1971) foi escolhida como a base do modelo proposto no CEB MC 90. Conforme MÜLLER \& HILSDORF (1991) esta relação foi adotada por duas razões. Primeiro, o modelo de SARGIN descreve muito bem o ramo ascendente da curva tensão-deformação; segundo, este modelo pode também ser usado como base para calcular diagramas tensão-deformação para estado multiaxial de tensões. Esta relação é mostrada na Fig. 4.2.

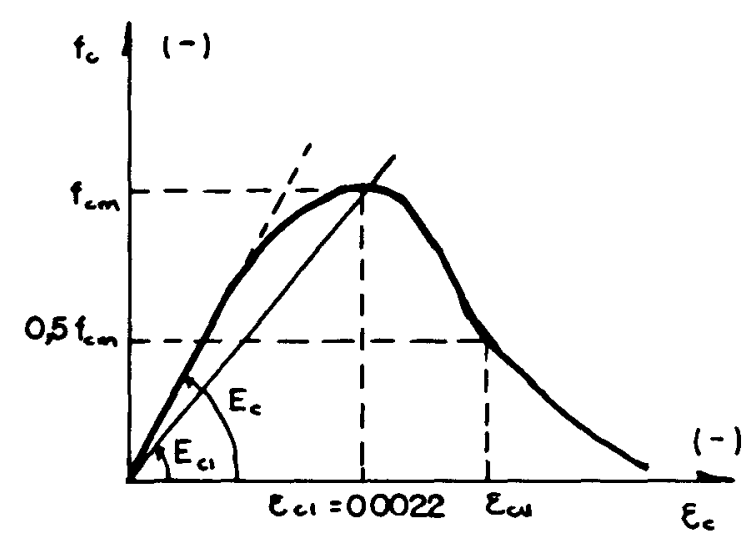

FIGURA 4.2 - Relação tensão-deformação para o concreto em compressão (CEB MC 90)

A relação $f_{c}-\varepsilon_{c}$ para a trecho ascendente e para o trecho descendente até $\left|f_{c}\right|>0,5 f_{c m}$ é dada pela seguinte equação: 


$$
f_{c}=f_{c m} \frac{\frac{E_{c}}{E_{c 1}} \frac{\varepsilon_{c}}{\varepsilon_{c 1}}-\left(\frac{\varepsilon_{c}}{\varepsilon_{c l}}\right)^{2}}{1+\left(\frac{E_{c}}{E_{c 1}}-2\right) \frac{\varepsilon_{c}}{\varepsilon_{c 1}}} \quad \text { para } \varepsilon>\varepsilon_{c u}
$$

onde

$\mathrm{E}_{\mathrm{c}}=$ módulo de elasticidade dado por:

$$
E_{c}=2.15 \times 10^{4}\left(f_{c m} / 10\right)^{1 / 3} \text { em (MPa) }
$$

$f_{c}=$ tensão de compressão uniaxial

$\varepsilon_{\mathrm{c}}=$ deformação por compressão

$\varepsilon_{\mathrm{cl}}=$ deformação correspondente à tensão máxima de compressão $\mathrm{f}_{\mathrm{cm}}$

$\mathrm{E}_{\mathrm{c} 1}=\mathrm{f}_{\mathrm{cm}} / 0,0022$ é o módulo de elasticidade secante da origem para o ponto de tensão máxima $f_{\mathrm{cm}}$

A deformação $\varepsilon_{c u}$ para $f_{c}=-0,5 f_{c m}$ pode ser calculada por:

$$
\varepsilon_{\mathrm{cu}}=\varepsilon_{\mathrm{c} 1}\left\{\left(\frac{1}{2} \frac{\mathrm{E}_{\mathrm{c}}}{\mathrm{E}_{\mathrm{cl}}}+1\right)+\left[\frac{1}{4}\left(\frac{1}{2} \frac{\mathrm{E}_{\mathrm{c}}}{\mathrm{E}_{\mathrm{c} 1}}+1\right)^{2}-\frac{1}{2}\right]^{1 / 2}\right\}
$$

Esta deformação $\varepsilon_{\mathrm{cu}}$ serve apenas para limitar a aplicação da equação 4.6.

Para $\varepsilon<\varepsilon_{\mathrm{cu}}$ a equação $\mathrm{f}_{\mathrm{c}}-\varepsilon_{\mathrm{c}}$ é dada por:

$$
f_{c}=-\left[\left(\frac{1}{\varepsilon_{\mathrm{cu}} / \varepsilon_{\mathrm{c} 1}} \xi-\frac{2}{\left(\varepsilon_{\mathrm{cu}} / \varepsilon_{\mathrm{cl}}\right)^{2}}\right)\left(\frac{\varepsilon_{\mathrm{c}}}{\varepsilon_{\mathrm{cc}}}\right)^{2}+\left(\frac{4}{\varepsilon_{\mathrm{cu}} / \varepsilon_{\mathrm{c} 1}}-\xi\right) \frac{\varepsilon_{\mathrm{c}}}{\varepsilon_{\mathrm{c} 1}}\right]^{-1}
$$

com 


$$
\xi=\frac{4\left(\left(\frac{\varepsilon_{\mathrm{cu}}}{\varepsilon_{\mathrm{cl}}}\right)^{2}\left(\frac{\varepsilon_{\mathrm{c}}}{\varepsilon_{\mathrm{c} 1}}-2\right)+2 \frac{\varepsilon_{\mathrm{cu}}}{\varepsilon_{\mathrm{c} 1}}-\frac{\mathrm{E}_{\mathrm{c}}}{\mathrm{E}_{\mathrm{cl}}}\right)}{\left(\frac{\varepsilon_{\mathrm{cu}}}{\varepsilon_{\mathrm{cl}}}\left(\frac{\mathrm{E}_{\mathrm{c}}}{\mathrm{E}_{\mathrm{c} 1}}-2\right)+1\right)^{2}}
$$

O CEB permite aproximar a equação 4.9 por uma reta.

Conforme observado por MÜLLER \& HILSDORF (1991) o trecho ascendente da curva de SARGIN fornece resultados compatíveis com dados experimentais, mas o trecho descendente é fortemente influenciado pelo comprimento da barra comprimida, uma vez que a fratura por compressão é um fenômeno discreto, isto é, em uma barra comprimida, a fratura não é distribuída uniformemente sobre o volume inteiro do material, mas é concentrada numa região fraturada de dimensão limitada.

A NBR-6118 adota como relação constitutiva para o concreto em compressão uniaxial, a expressão parabólica proposta por HOGNESTAD (1951) modificada pela adoção do tramo constante igual a $f_{c m}$ para deformações inferiores a $\varepsilon_{0}$ (Fig. 4.3).

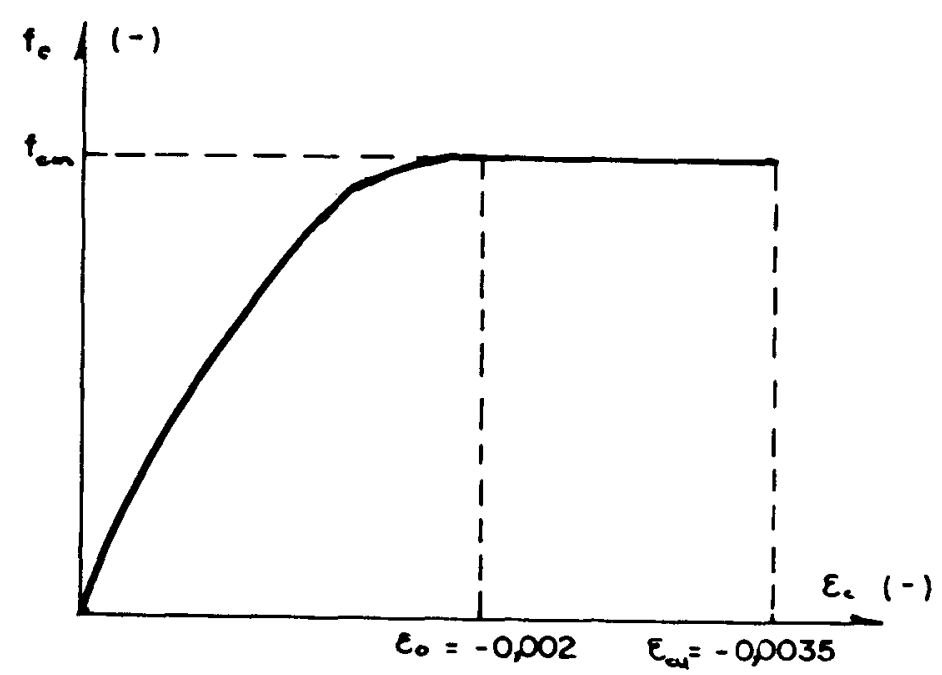

FIGURA 4.3 - Relação tensão-deformação para o concreto em compressão (NBR-6118) 
A equação correspondente é dada por:

$$
f_{c}=-f_{c m}\left[2\left(\frac{\varepsilon_{c}}{\varepsilon_{0}}\right)-\left(\frac{\varepsilon_{c}}{\varepsilon_{0}}\right)^{2}\right] \quad \text { se } \varepsilon>\varepsilon_{0}
$$

e

$$
\mathrm{f}_{\mathrm{c}}=-\mathrm{f}_{\mathrm{cm}} \quad \text { se } \varepsilon<\varepsilon_{0}
$$

onde

$\mathrm{f}_{\mathrm{cm}}=$ valor médio da resistência à compressão obtida em ensaios uniaxiais de tensão

$\varepsilon_{0}=$ deformação correspondente à tensão máxima $f_{\mathrm{cm}}\left(\varepsilon_{0} \cong-0,002\right)$

$\varepsilon_{\mathrm{c}}=$ deformação no concreto

$f_{c}=$ tensão no concreto

Admite-se para o concreto um comportamento elástico não-linear para $\varepsilon>\varepsilon_{0}$ e plástico para $\varepsilon<\varepsilon_{0}$.

Para o concreto em tração uniaxial, é possível relacionar a resistência média à tração com a resistência à compressão. O CEB MC 90 fornece a equação:

$$
f_{c t m}=0,30 f_{c k}^{2 / 3} \quad \text { em MPa }
$$

e a NBR-6118 propõe:

$$
f_{\mathrm{tk}}=\frac{f_{\mathrm{ck}}}{10} \quad \text { em MPa para } f_{\mathrm{ck}} \leq 18 \mathrm{MPa}
$$




$$
f_{\text {tk }}=0,06 f_{c k}+0,7 \text { em MPa para } f_{c k}>18 \mathrm{MPa}
$$

Para a verificação da capacidade resistente do concreto armado, em geral, não se considera a sua resistência à tração, a qual, no entanto, é um parâmetro importante na verificação do estado limite de utilização.

O CEB MC 90 propõe uma relação tensão-deformação para o concreto tracionado não-fissurado, dada por (Fig. 4.4):

$$
\begin{array}{ll}
f_{c}=E_{c} \varepsilon_{c} & \text { para } f_{c}<0,9 f_{c t m} \\
f_{c}=f_{c t m}-\frac{0,1 f_{c t m}}{0,00015-\frac{0,9 f_{c t m}}{E_{c}}}\left(0,00015-\varepsilon_{c t}\right) \text { para } f_{c}>0,9 f_{c t m}
\end{array}
$$

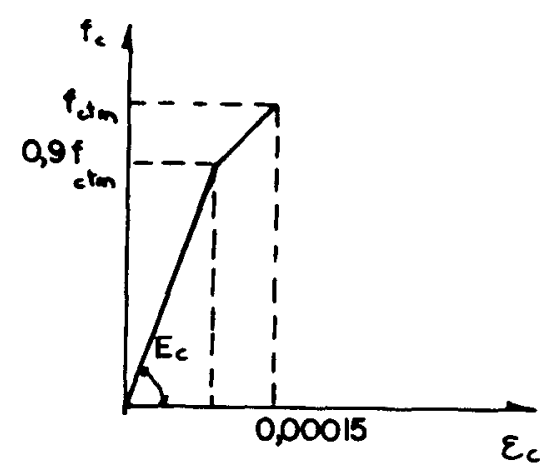

FIGURA 4.4 - Relação tensão-deformação para o concreto em tração (CEB MC 90)

A NBR-6118 não apresenta relação constitutiva para o concreto em tração.

Neste trabalho, adota-se como relação constitutiva tensão-deformação aquela recomendada pela NBR-6118 para o concreto em compressão (equações 4.11 e 4.12) e para o concreto não fissurado em tração, a equação 
proposta pelo CEB MC 90 (equações 4.13 e 4.14). Não se considera a resistência à tração do concreto após a fissuração, isto é, despreza-se o efeito do enrijecimento devido à presença de concreto intacto entre as fissuras ("tension stiffening").

Deve-se ressaltar que o diagrama tensão-deformação do concreto é influenciado tanto por sua idade, que afeta sua resistência e retração, quanto pela duração do carregamento, que influencia sua deformação lenta (creep).

Com relação aos valores de $\mathrm{f}_{\mathrm{cm}}$, recomenda-se utilizar os valores médios, obtidos em ensaios uniaxiais de tensão, pois eles representam melhor o comportamento mais provável dos materiais, conforme salienta SOLER (1995).

O CEB-FIP MC 90 recomenda que o $f_{c m}$ seja estimado por:

$$
f_{c m}=f_{c k}+\Delta f
$$

onde $\Delta \mathrm{f}=\mathbf{8} \mathrm{MPa}$.

A NBR-6118 recomenda que $f_{c m}$ seja estimado por:

$$
f_{c m}=f_{c k}+1,645 s
$$

onde s é o desvio padrão da amostra de corpos de prova ensaiados, que dependerá do tipo de controle na produção do concreto, e varia de 2 a $7 \mathrm{MPa}$.

\subsubsection{Equações Constitutivas para o Aço}

Para as armaduras do concreto armado a NBR-6118 adota para o aço classe A o diagrama mostrado na Fig. 4.5. 


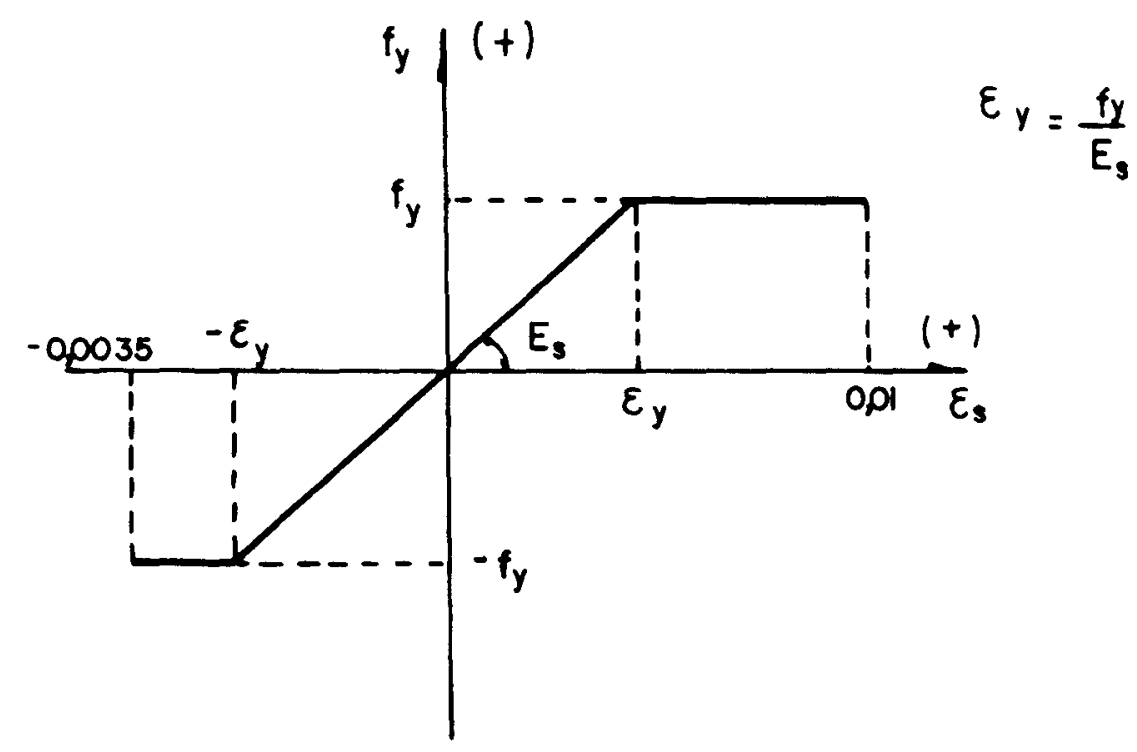

FIGURA 4.5 - Relação tensão-deformação para aço classe A (NBR-6118)

A equação constitutiva é dada por:

$$
\mathrm{f}_{\mathrm{s}}=\mathrm{E}_{\mathrm{s}} \varepsilon_{\mathrm{s}} \quad \text { para }-\varepsilon_{\mathrm{y}}<\varepsilon_{\mathrm{s}}<\varepsilon_{\mathrm{y}}
$$

onde

$\mathrm{f}_{\mathrm{s}} \quad=$ tensão na armadura

$\varepsilon_{\mathrm{s}} \quad=$ deformação da armadura

$\mathrm{E}_{\mathrm{s}} \quad=$ módulo de elasticidade do aço

$\mathrm{f}_{\mathrm{y}} \quad=$ tensão de escoamento da armadura

$\varepsilon_{\mathrm{y}}=$ deformação correspondente à tensão de escoamento

Para o aço classe B a NBR-6118 recomenda a utilização de curva experimental que contenha a resistência característica $f_{\mathrm{yk}}$ calculada elasticamente com base em ensaios realizados em laboratório idôneo. $\mathrm{Na}$ falta desta curva experimental, permite-se adotar o diagrama simplificado da Fig. 4.6. 


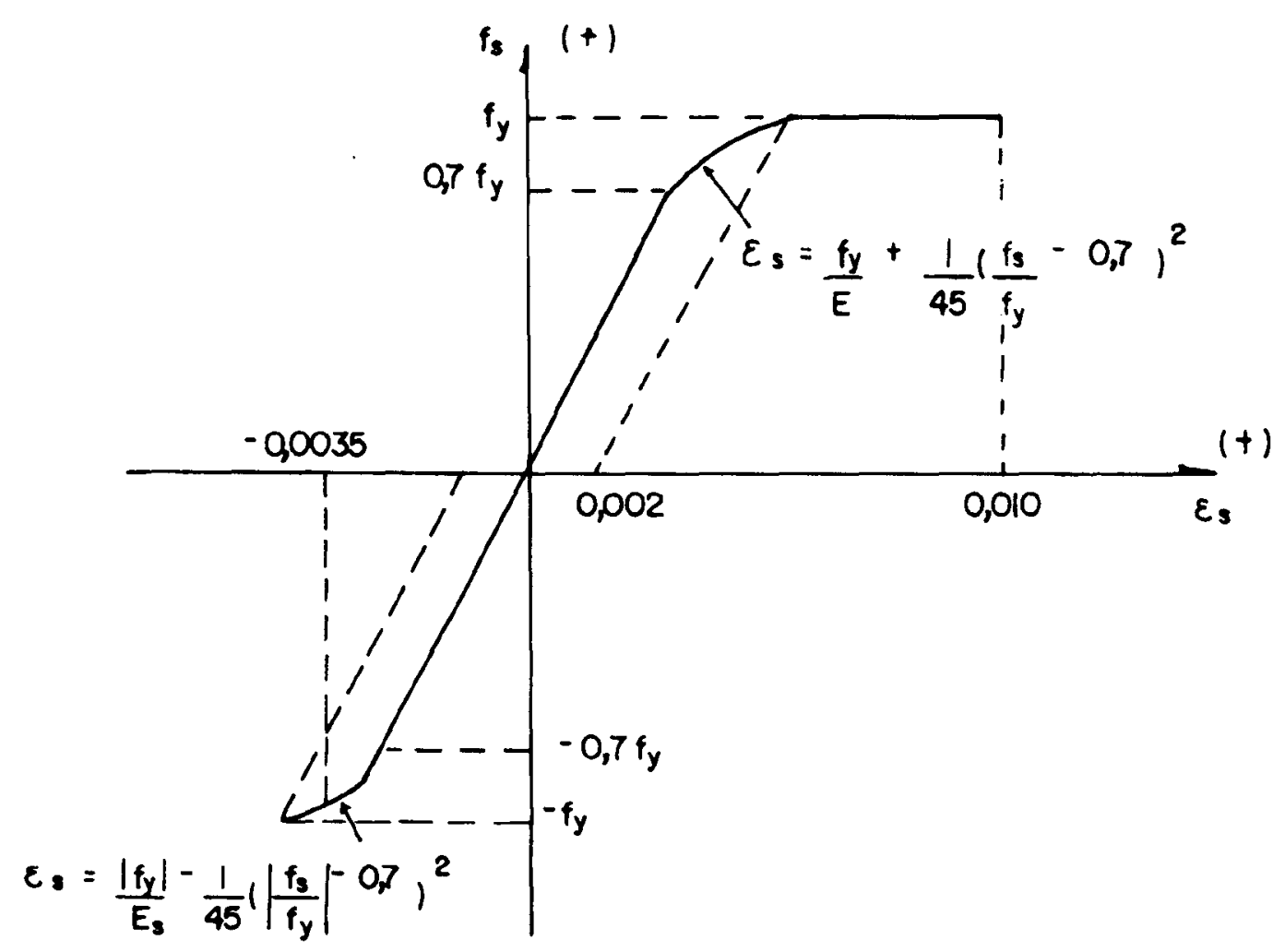

FIGURA 4.6 - Relação tensão-deformação para aço classe B (NBR-6118)

O comportamento do aço é elasto-plástico com tensão de escoamento igual a $\mathrm{f}_{\mathrm{y}}$. $\mathrm{O}$ módulo de elasticidade é considerado constante e igual a 210.000 MPa.

Neste trabalho será adotada a equação constitutiva do aço classe A (eq. 4.20) e admite-se uma perfeita aderência entre a armadura e o concreto $\left(\varepsilon_{\mathrm{c}}=\right.$ $\left.\varepsilon_{\mathrm{s}}\right)$.

\subsection{Programa para o Cálculo Automático}

Com a finalidade de se automatizar os procedimentos de cálculo descritos nos capítulos 3 e 4, para a obtenção dos deslocamentos, esforços solicitantes, reações de apoio e deformações plásticas no pórtico plano de concreto armado, foi elaborado o programa "PF" em linguagem FORTRAN 
77, compilado no FORTRAN "POWER STATION", para utilização em microcomputadores. $\mathrm{O}$ programa permite considerar ou não a resistência do concreto à tração. Pode-se, com uma pequena alteração no programa, considerar qualquer relação constitutiva para o concreto e para o aço.

A entrada de dados é feita em arquivo sequencial formatado (DADOSPF), onde deve-se usar um sistema de unidades compatível. Os limites de utilização do programa dependerão da disponibilidade de memória auxiliar em disco. Como está compilado atualmente, permite processar estruturas com até 400 nós e 400 elementos. A saída de resultados e feita em arquivo sequencial formatado (SAIDA-PF), onde são impressos os dados de entrada, os deslocamentos nodais, esforços solicitantes, reações de apoio e deformações plásticas em cada fatia de cada elemento.

As cargas devem ser aplicadas nos nós. Carregamentos, deslocamentos e esforços são positivos no sentido do sistema de coordenadas apresentado na Fig. 4.7.

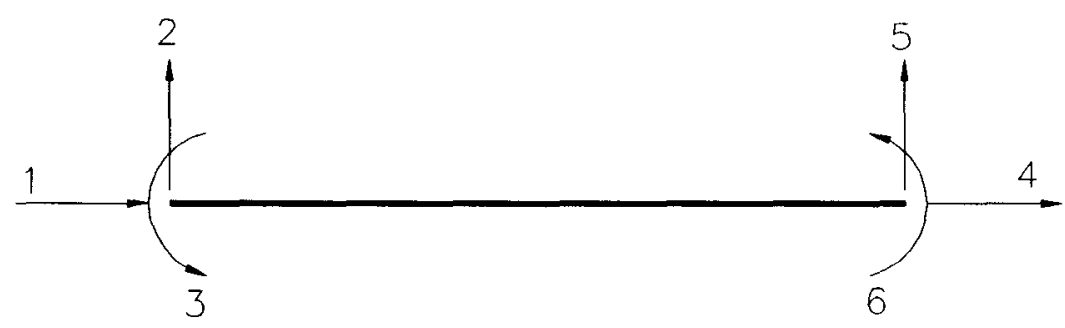

FIGURA 4.7 - Sistema local de coordenadas

A seguir, são mostrados os fluxogramas simplificados do programa principal e das principais subrotinas. 


\subsubsection{Fluxogramas Simplificados}

\subsubsection{Programa Principal}

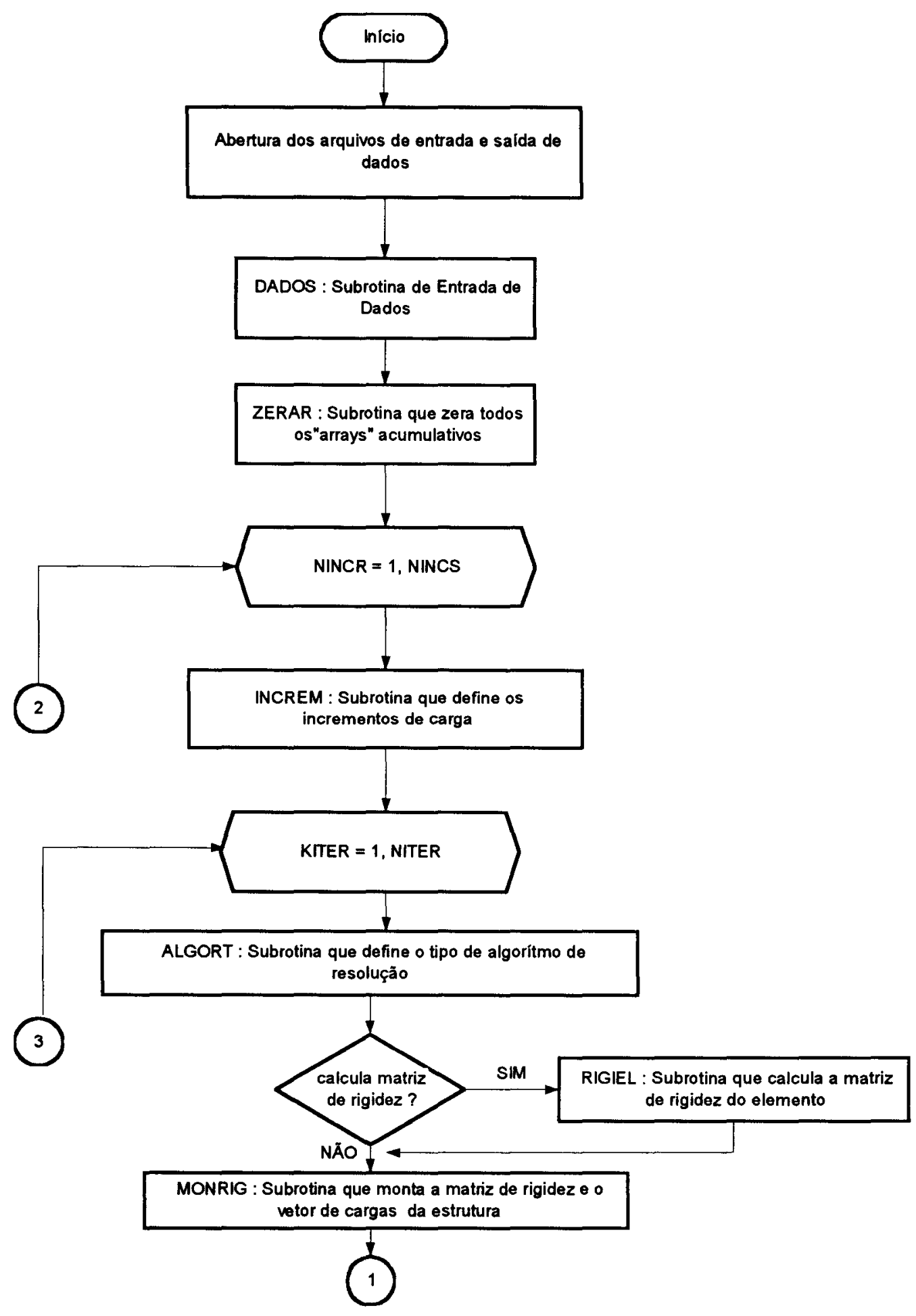




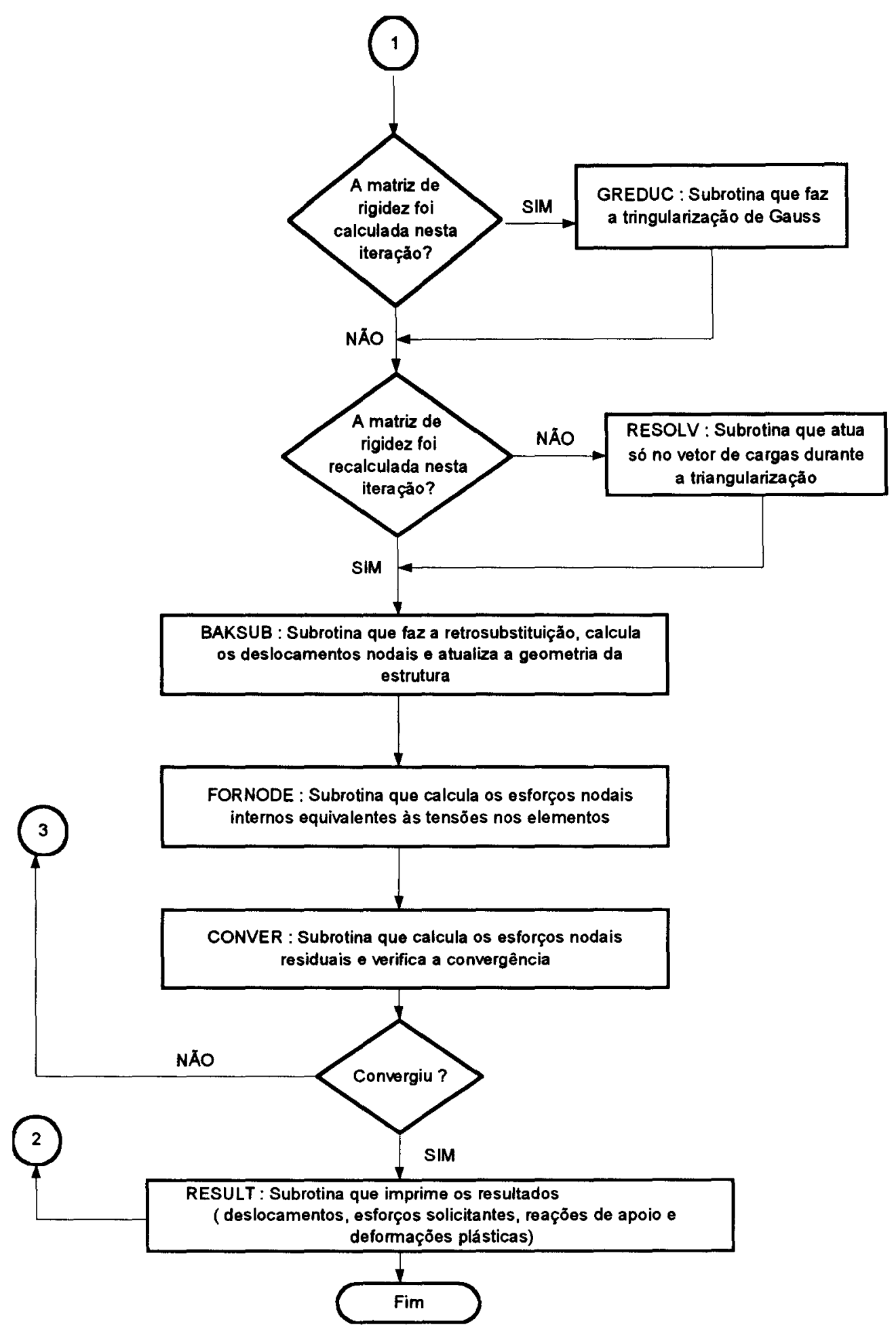




\subsubsection{Subrotina DADOS}

Esta subrotina lê e imprime todos os parâmetros da estrutura e grava no arquivo DADOS-PF.

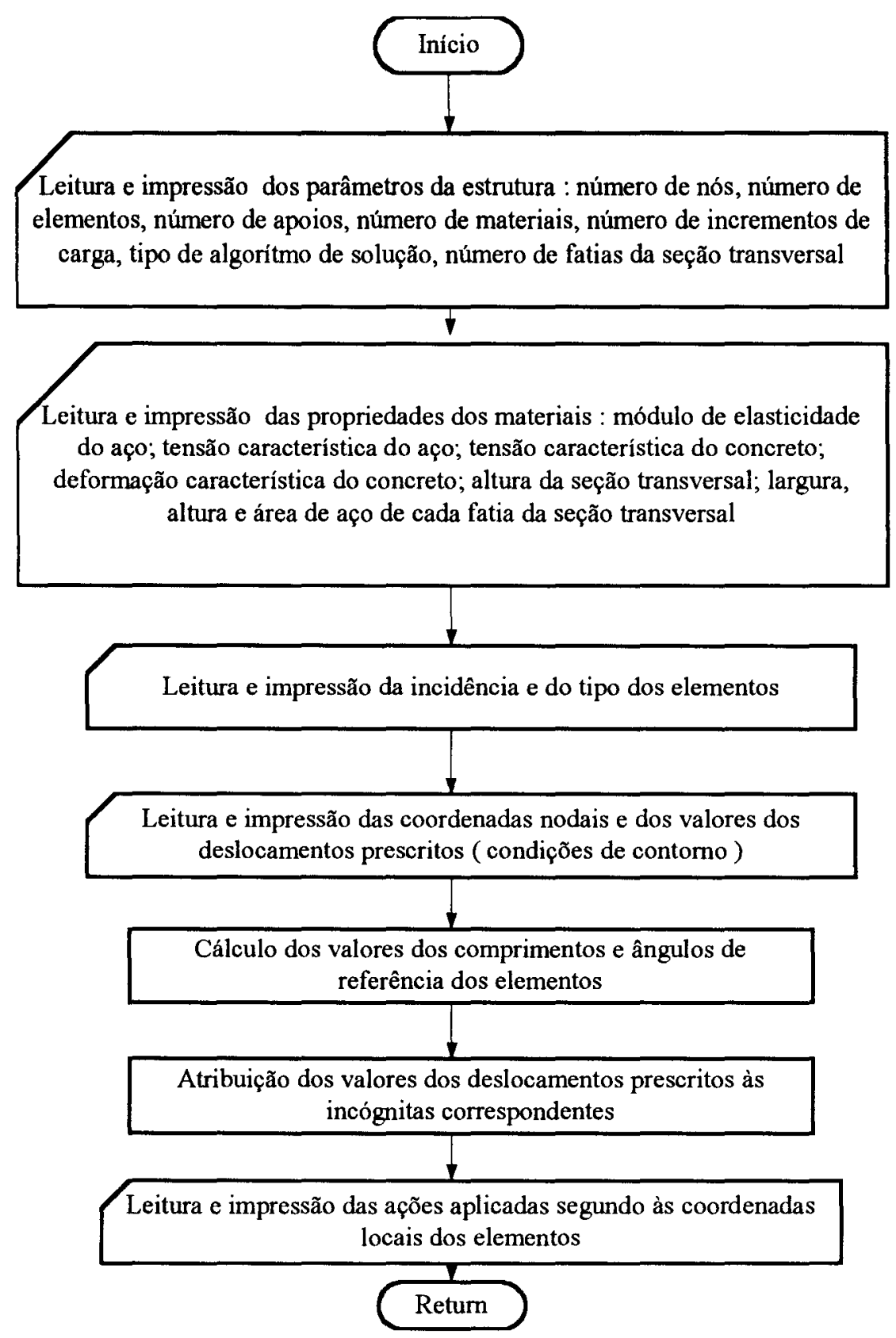

\subsubsection{Subrotina ZERAR}

Nesta subrotina são zerados todos os "arrays" acumulativos do programa. 


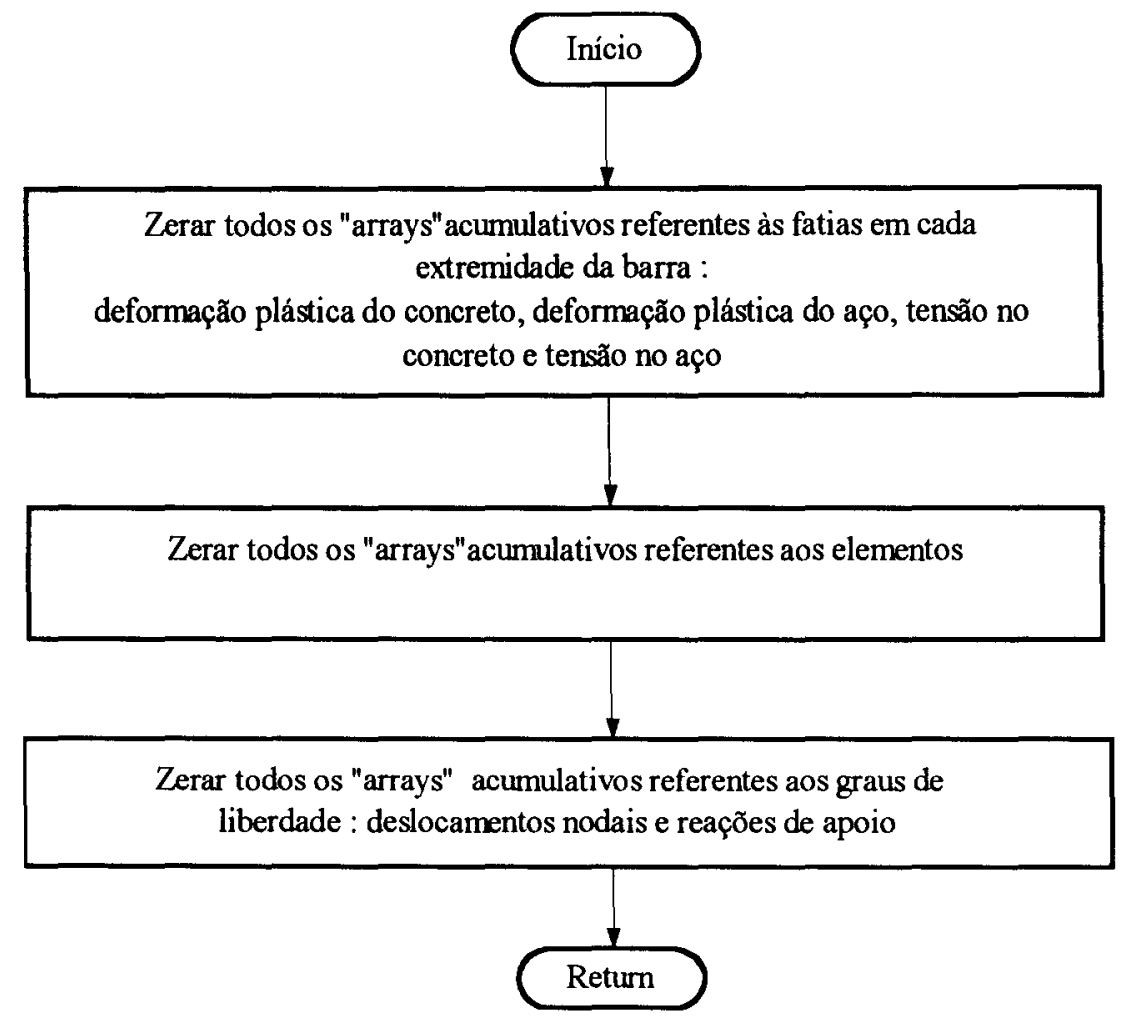

\subsubsection{Subrotina ICREM}

É a subrotina responsável pelo controle dos incrementos de carga e pela atualização do vetor de cargas em cada passo do processo incremental.

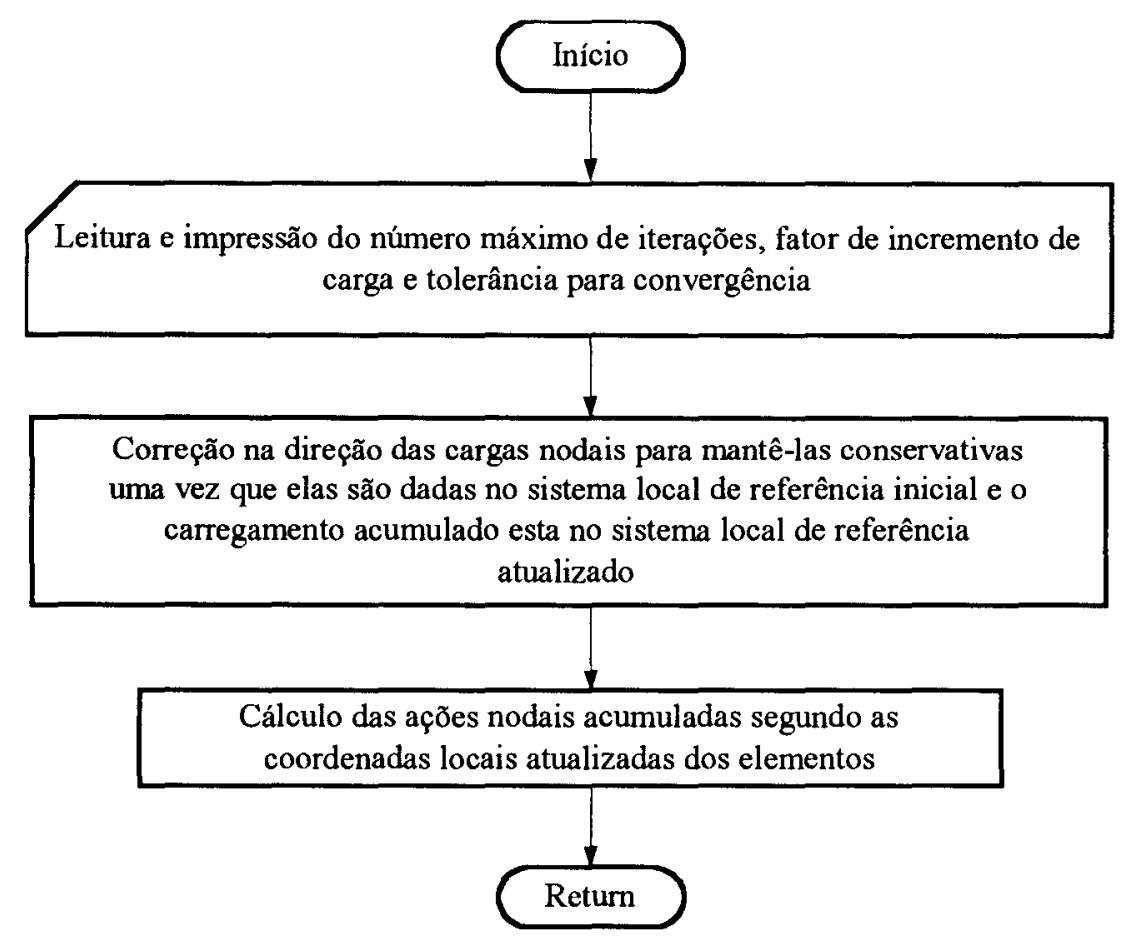




\subsubsection{Subrotina RIGIEL}

Nesta subrotina calcula-se a matriz de rigidez tangente de cada elemento, considerando-se a não-linearidade geométrica e do material. Utilizase a subrotina FATIA para obtenção dos coeficientes de rigidez da seção tranversal.

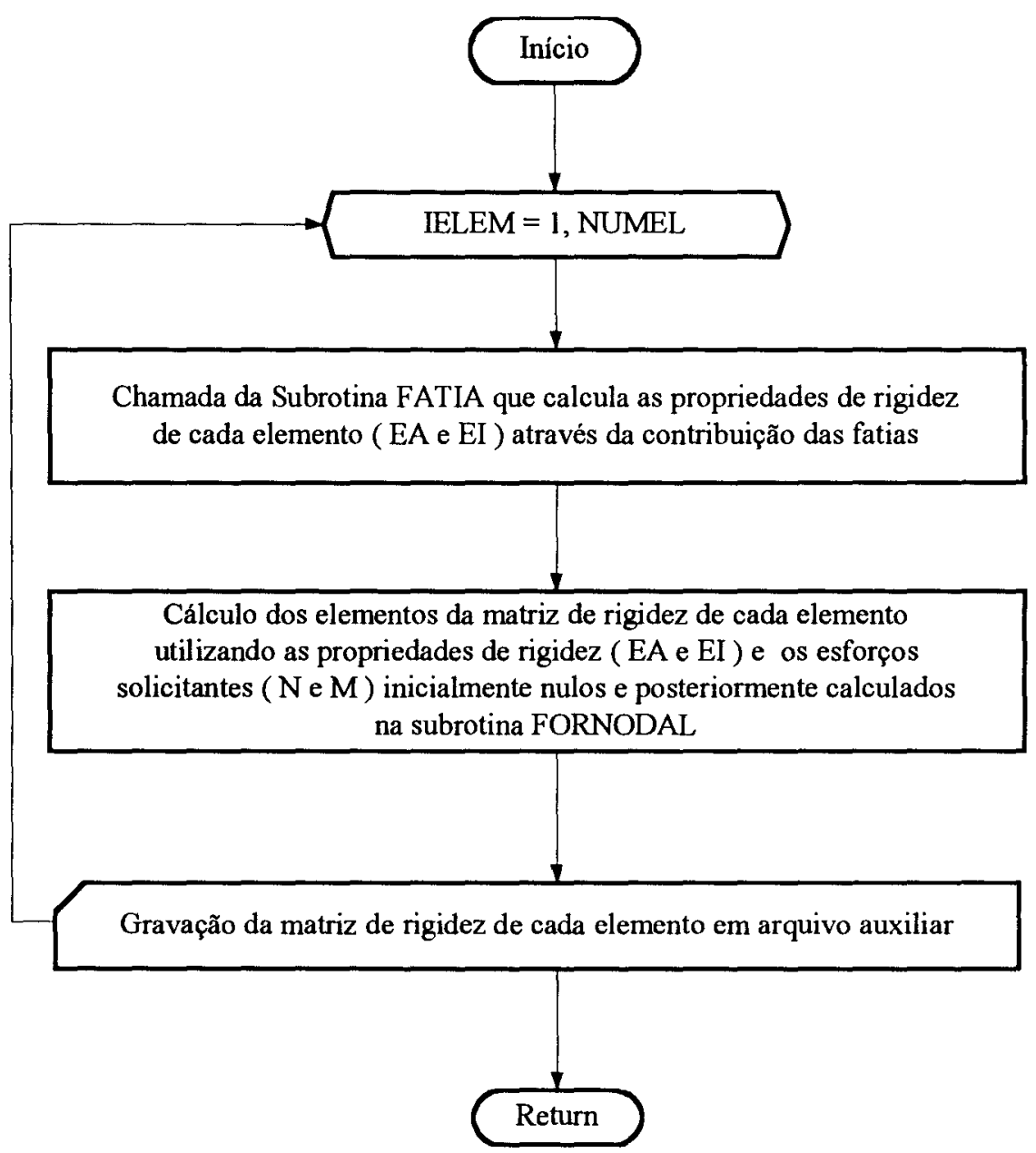




\subsubsection{Subrotina FATIA}

Esta subrotina calcula a contribuição das fatias nos valores de EA, EAY e EI da seção tranversal em cada extremidade do elemento assim como os valores médios de EA, EAY e EI.

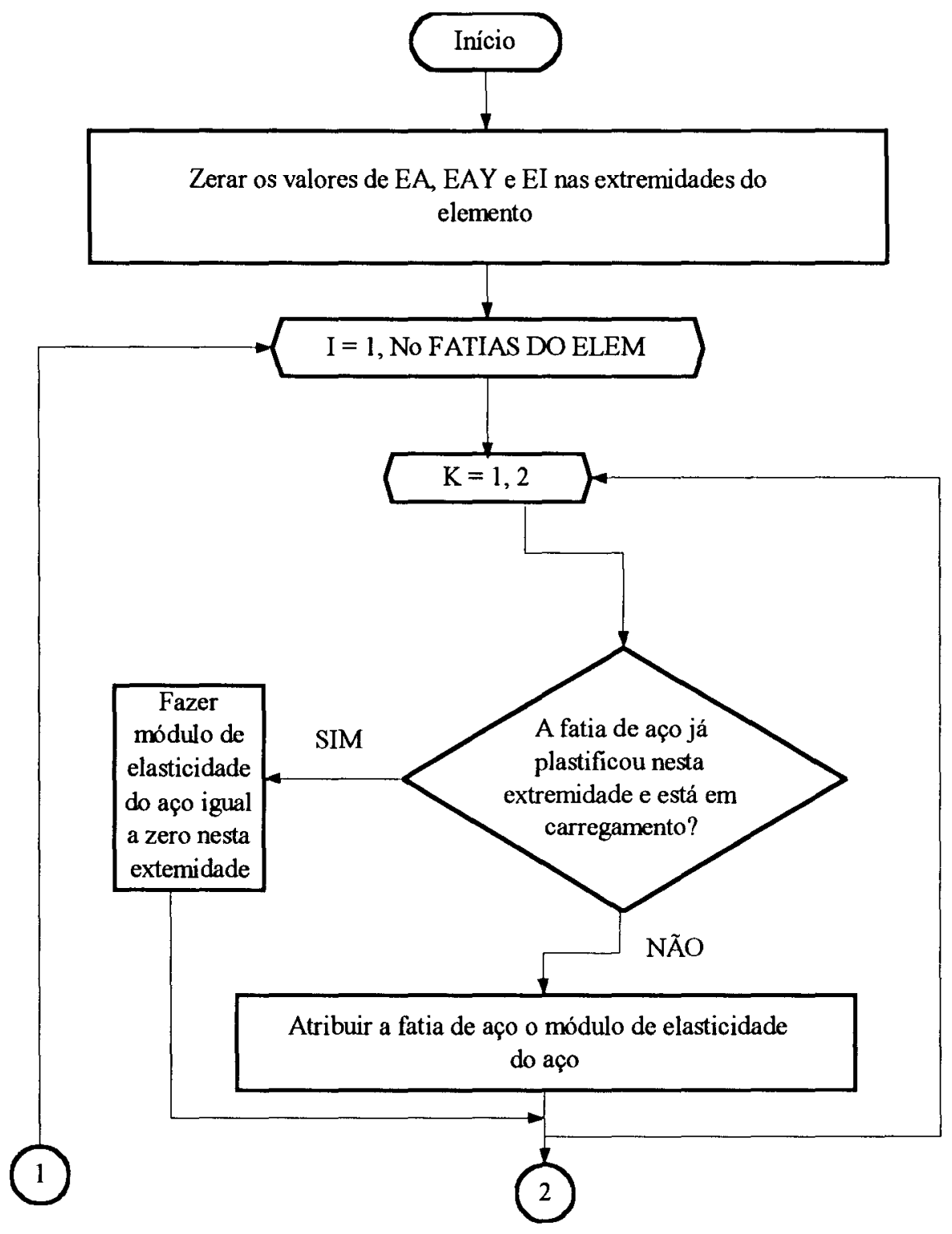




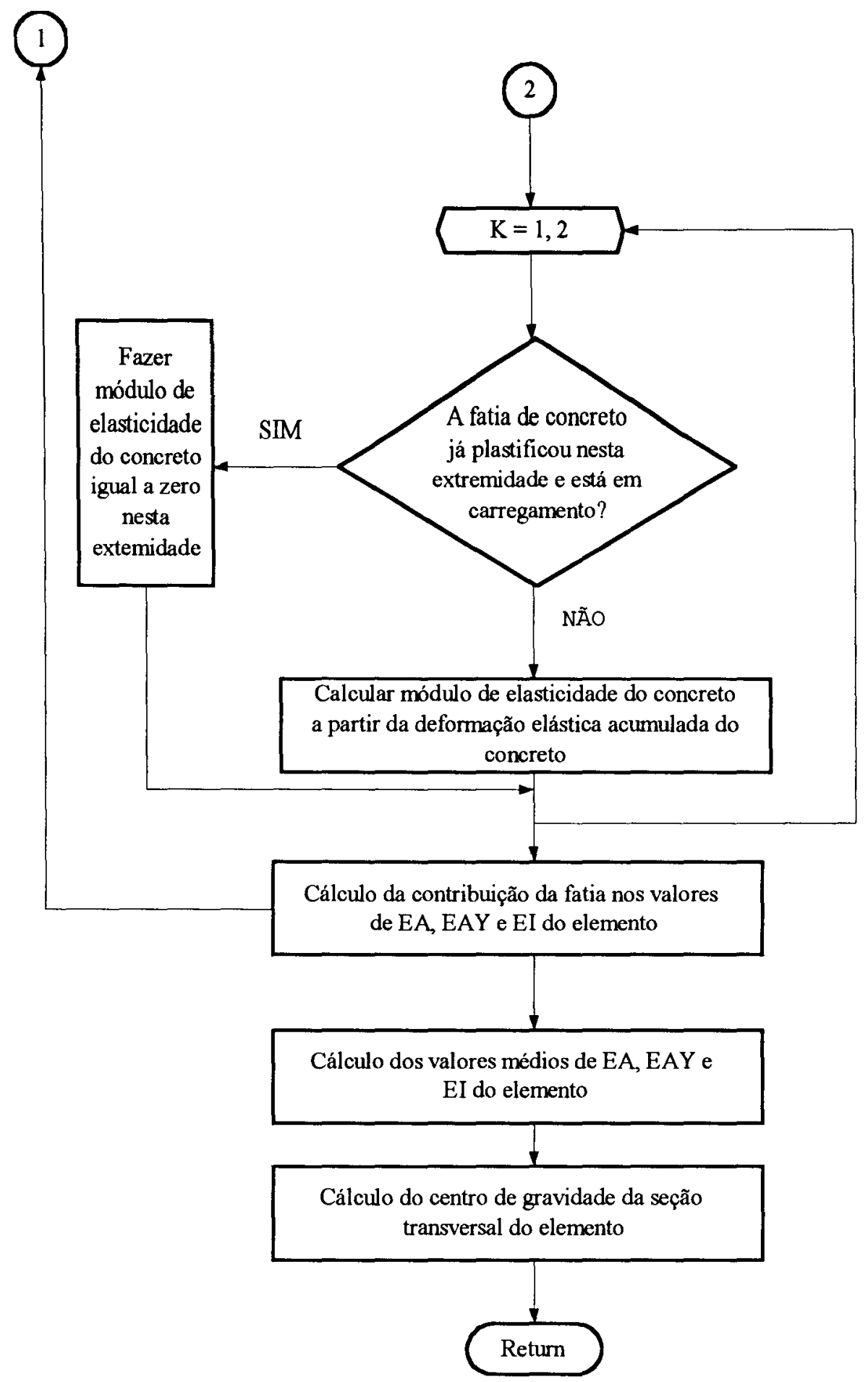




\subsubsection{Subrotina MONRIG}

Esta subrotina monta a matriz de rigidez tangente da estrutura, através da contribuição de cada elemento, assim como o vetor de cargas nodais da estrutura. Essa matriz é armazenada no arquivo auxiliar RIGIT.

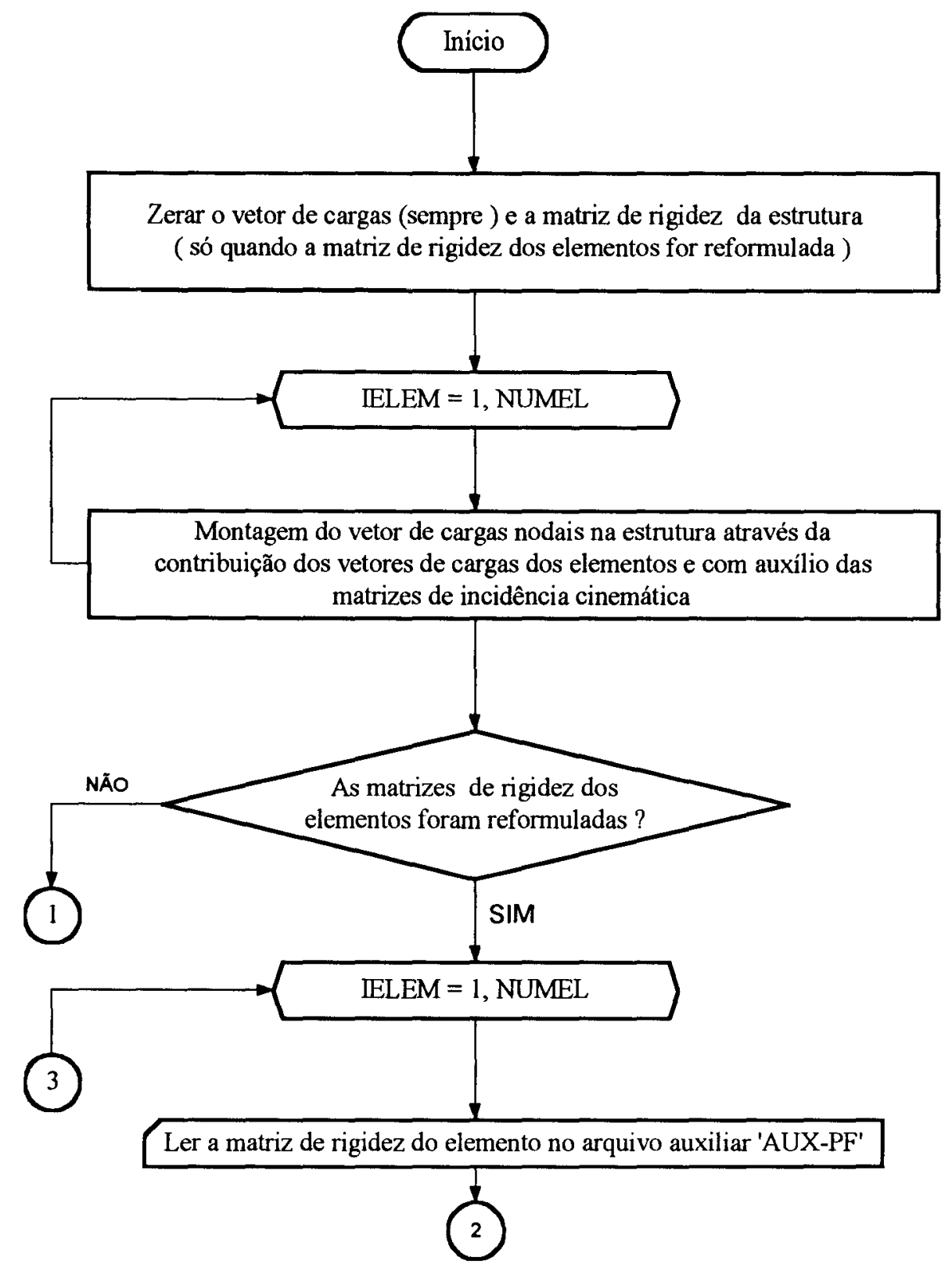




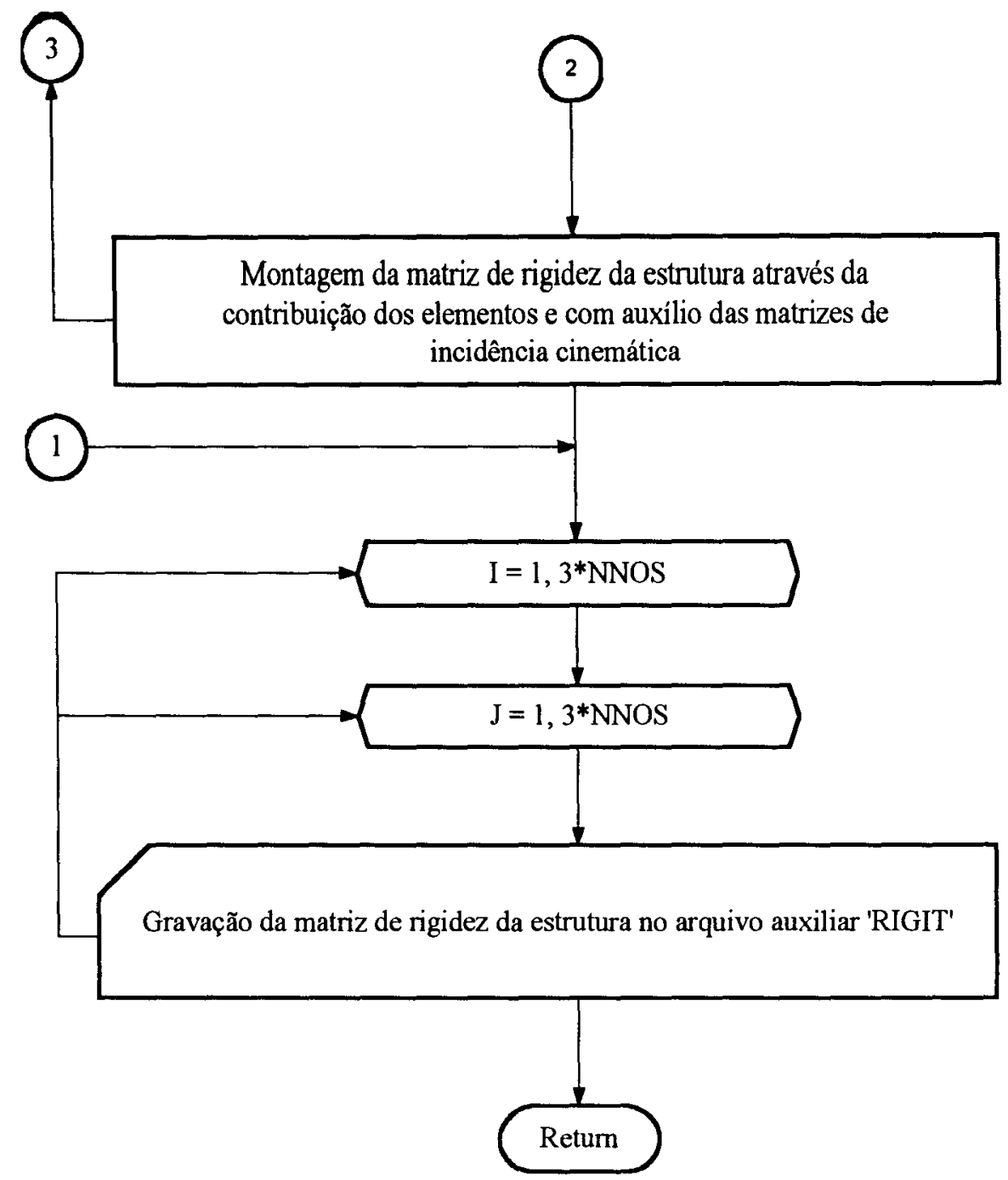

\subsubsection{Subrotina FORNODAL}

Nesta subrotina são calculados os esforços nodais internos equivalentes nos elementos, através da integração das tensões nas fatias. As relações constitutivas do concreto e do aço são consideradas. Nesta subrotina calculamse os esforços solicitantes nas extremidades de cada elemento. 


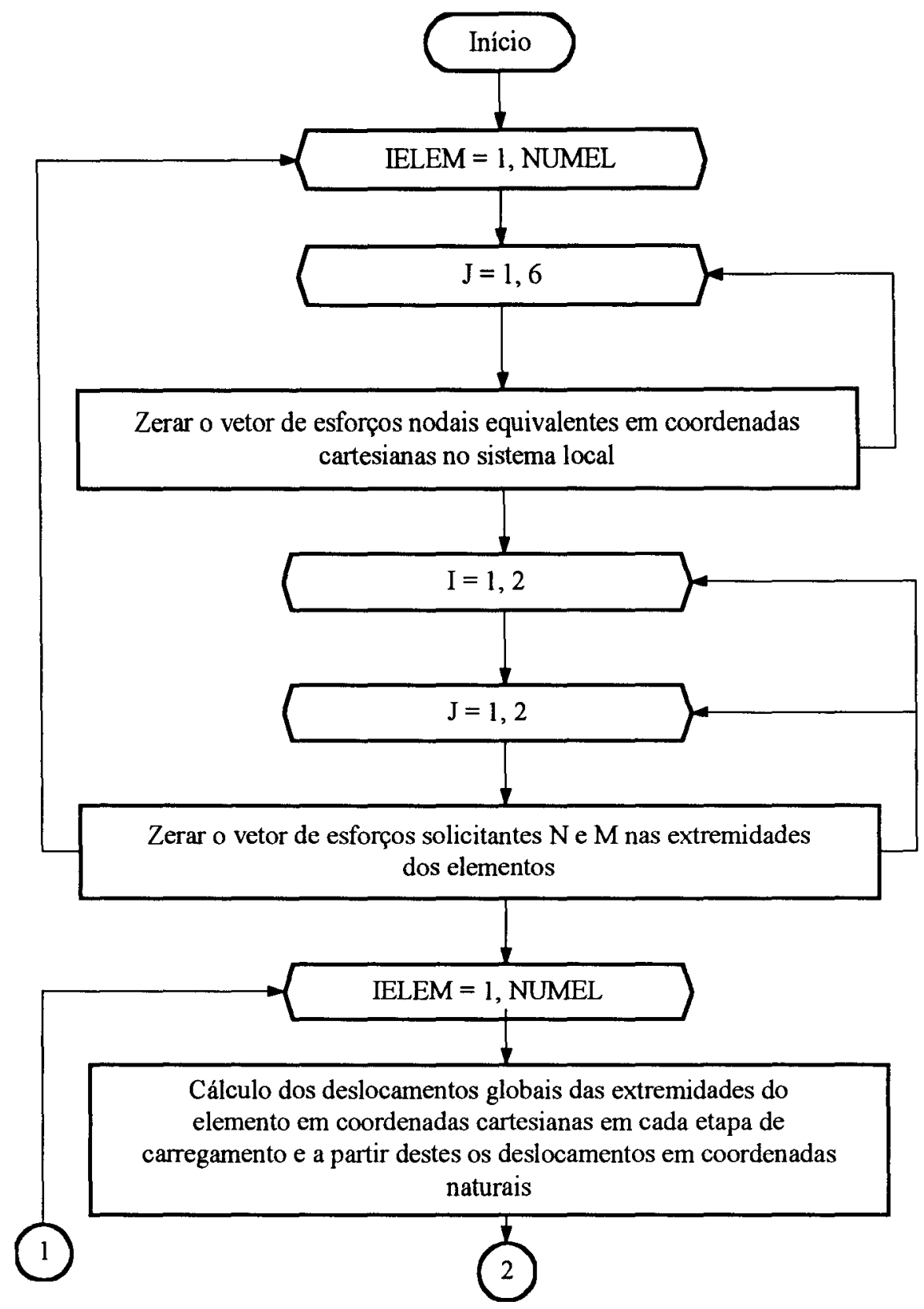




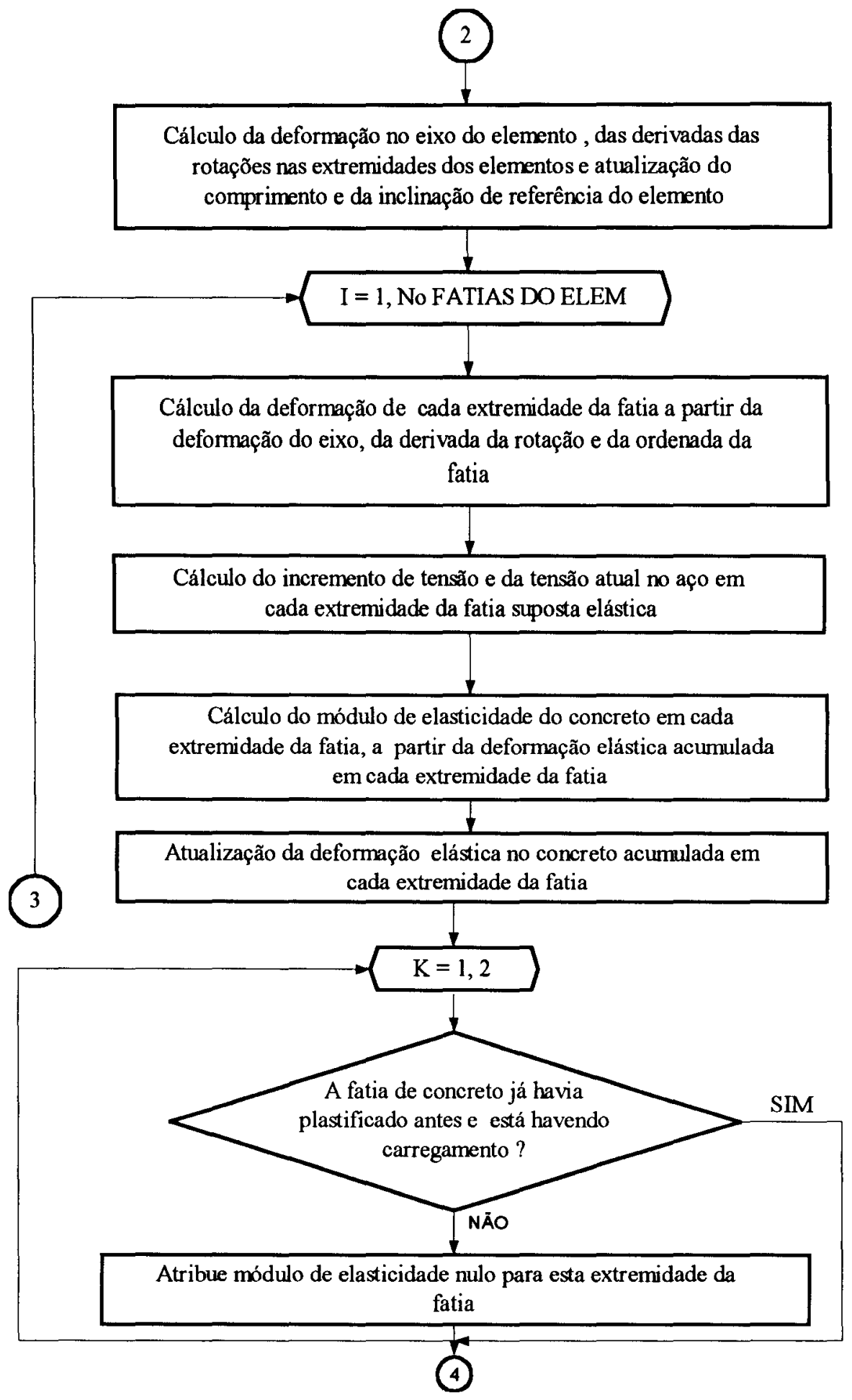




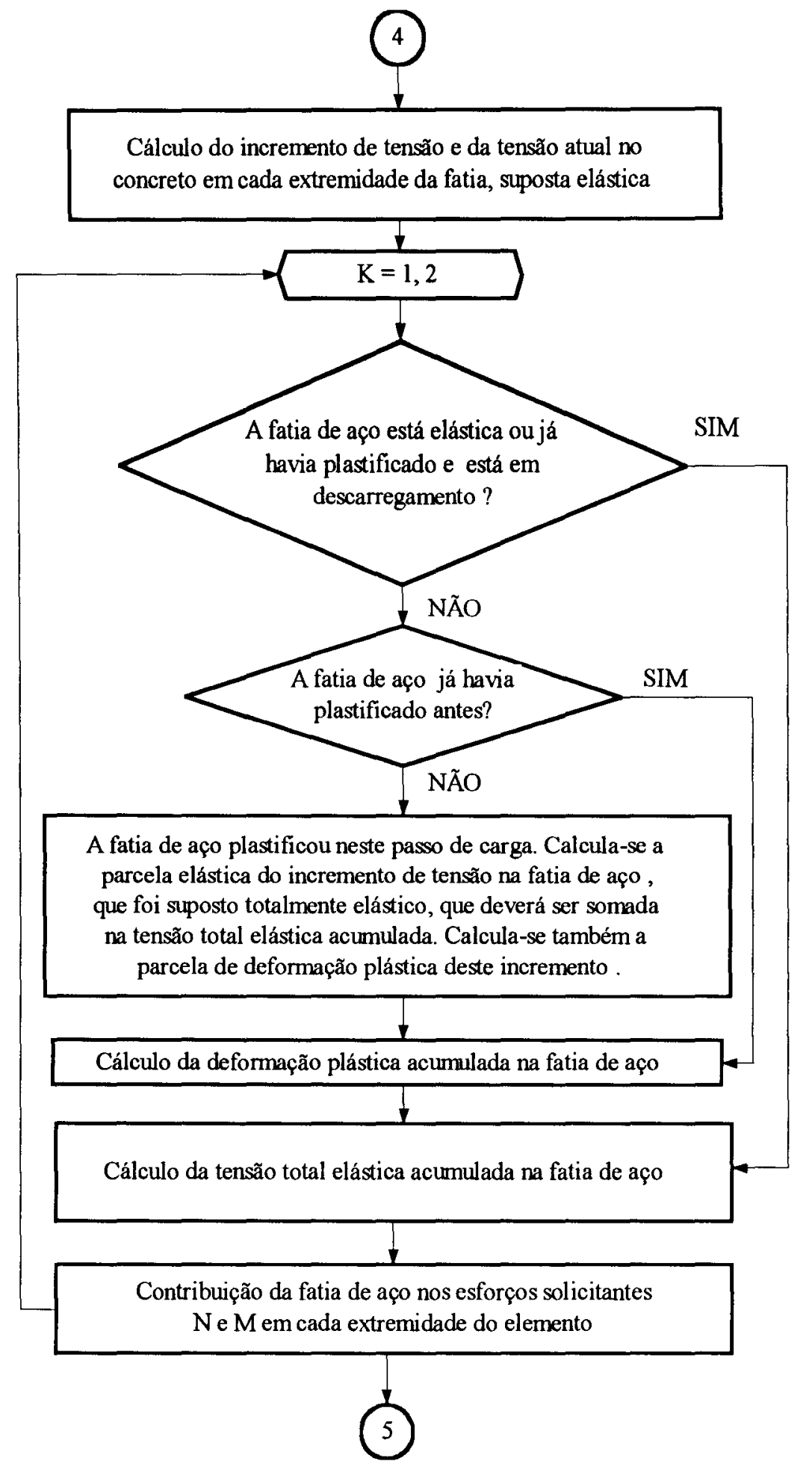




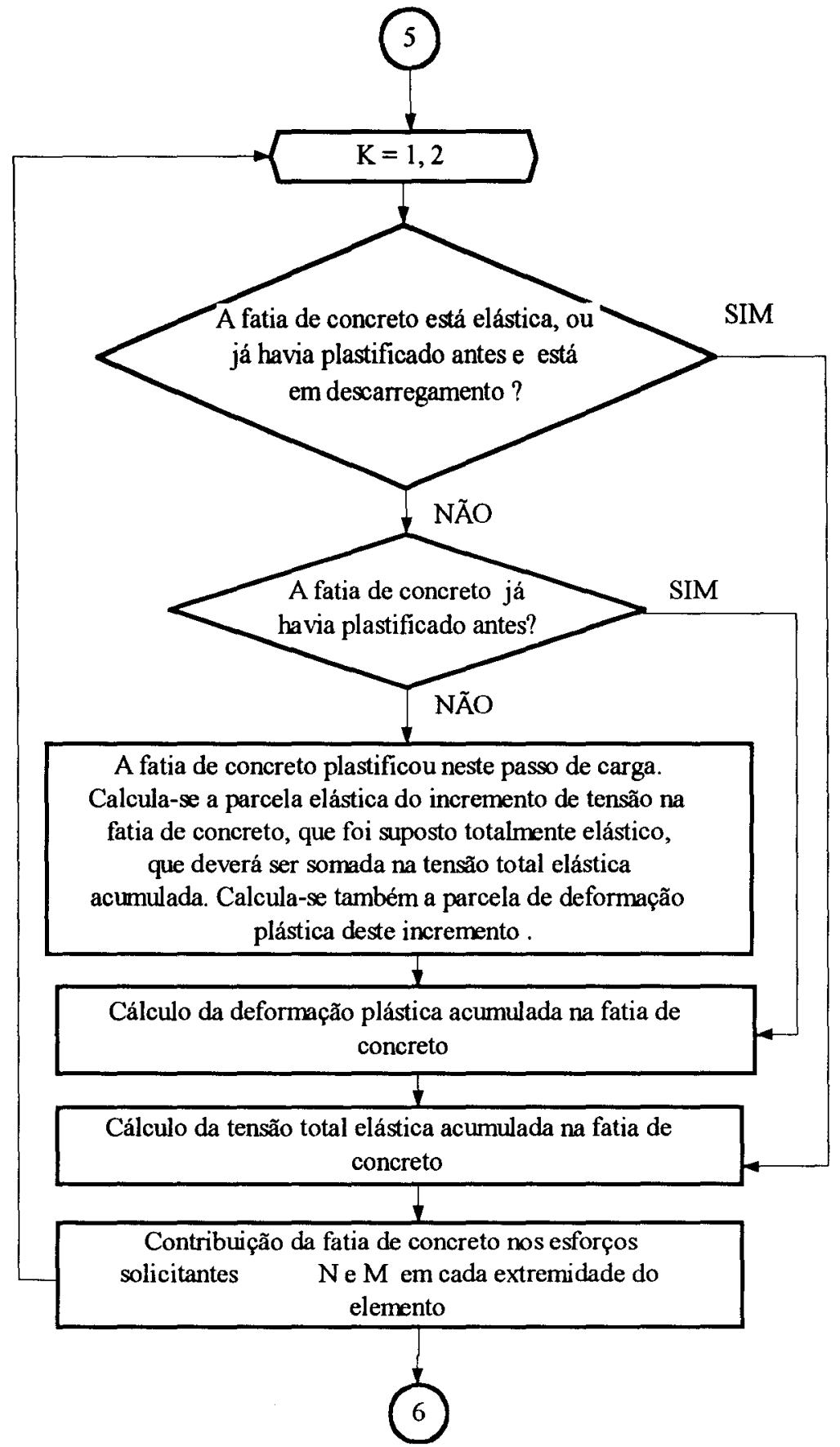




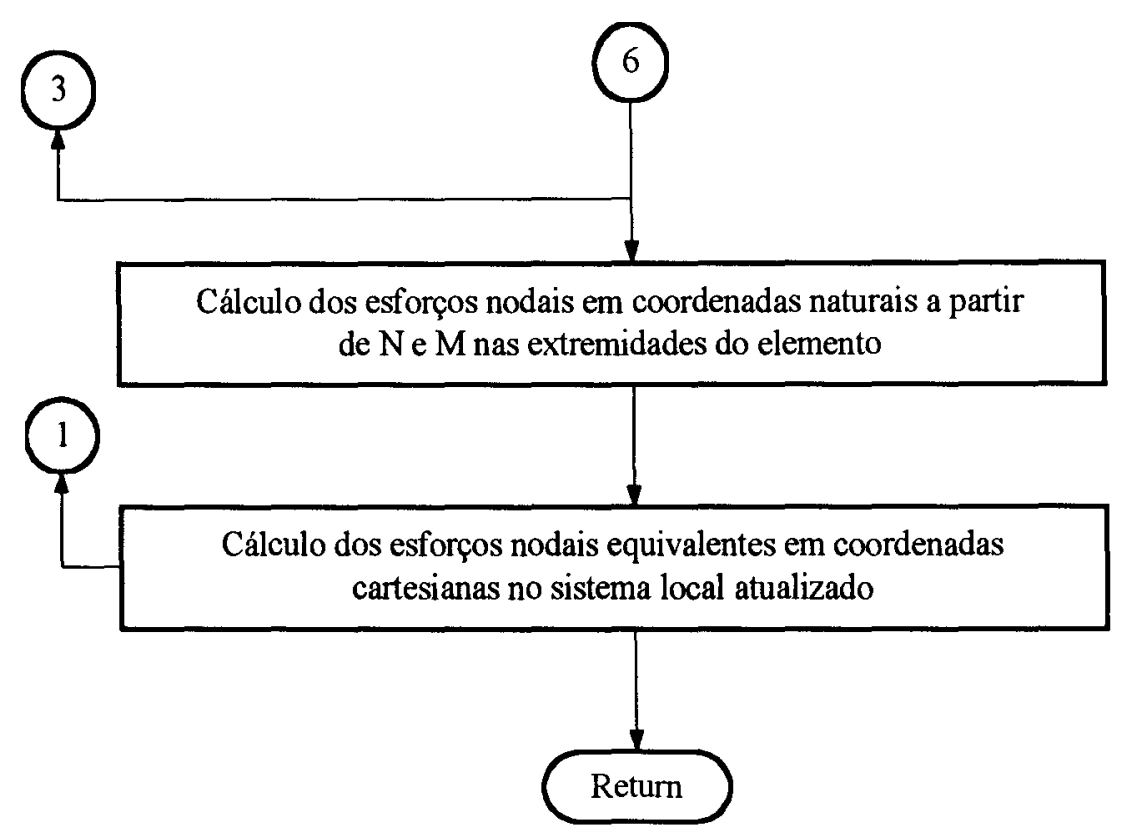

\subsubsection{Subrotina CONVER}

Nesta subrotina calculam-se os esforços nodais residuais para cada elemento, os quais se constituirão no carregamento a ser aplicado na iterą̧ão seguinte, caso não ocorra a convergência na iteração atual. Nela verifica-se também a convergência da solução, utilizando-se o critério dos deslocamentos ou dos esforços residuais. 


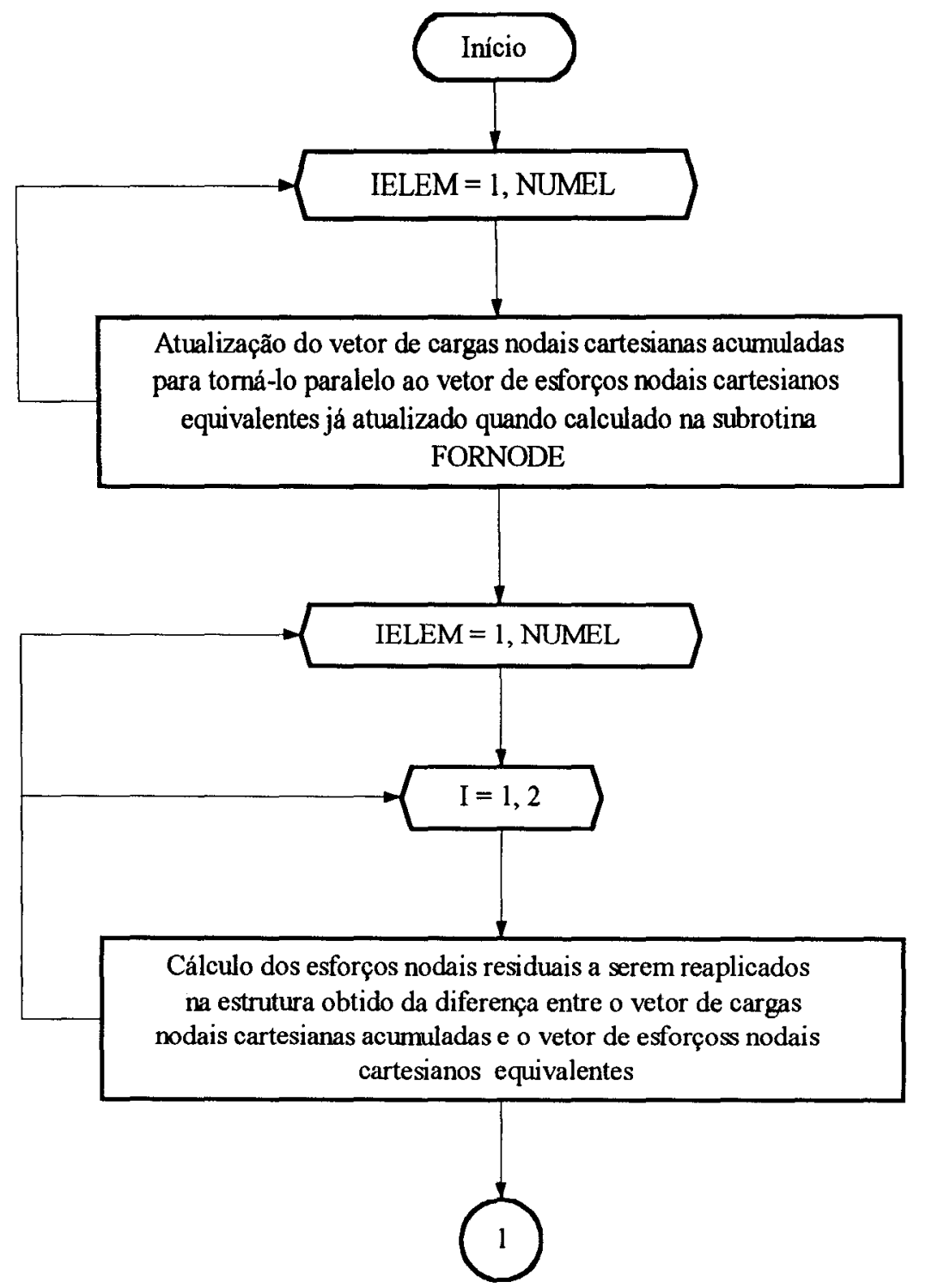




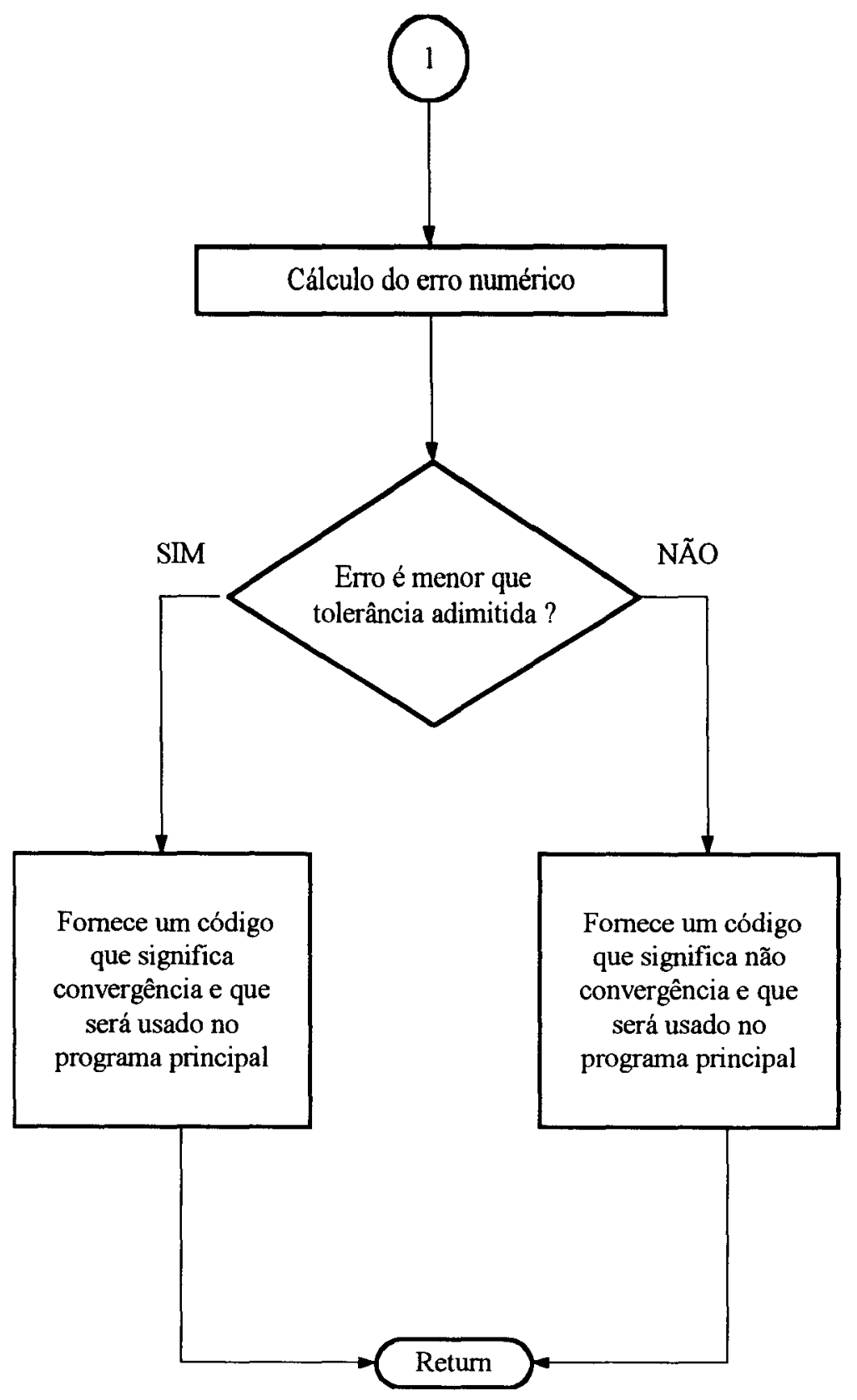

\subsubsection{Subrotina RESULT}

Nesta subrotina são impressos os resultados da análise que, no caso, são os deslocamentos nodais, as reações de apoio, os esforços nas extremidades dos elementos e as deformações plásticas nas extremidades de cada fatia de cada elemento. Estes resultados podem ser impressos para cada iteração ou para cada incremento de carga após a convergência. 


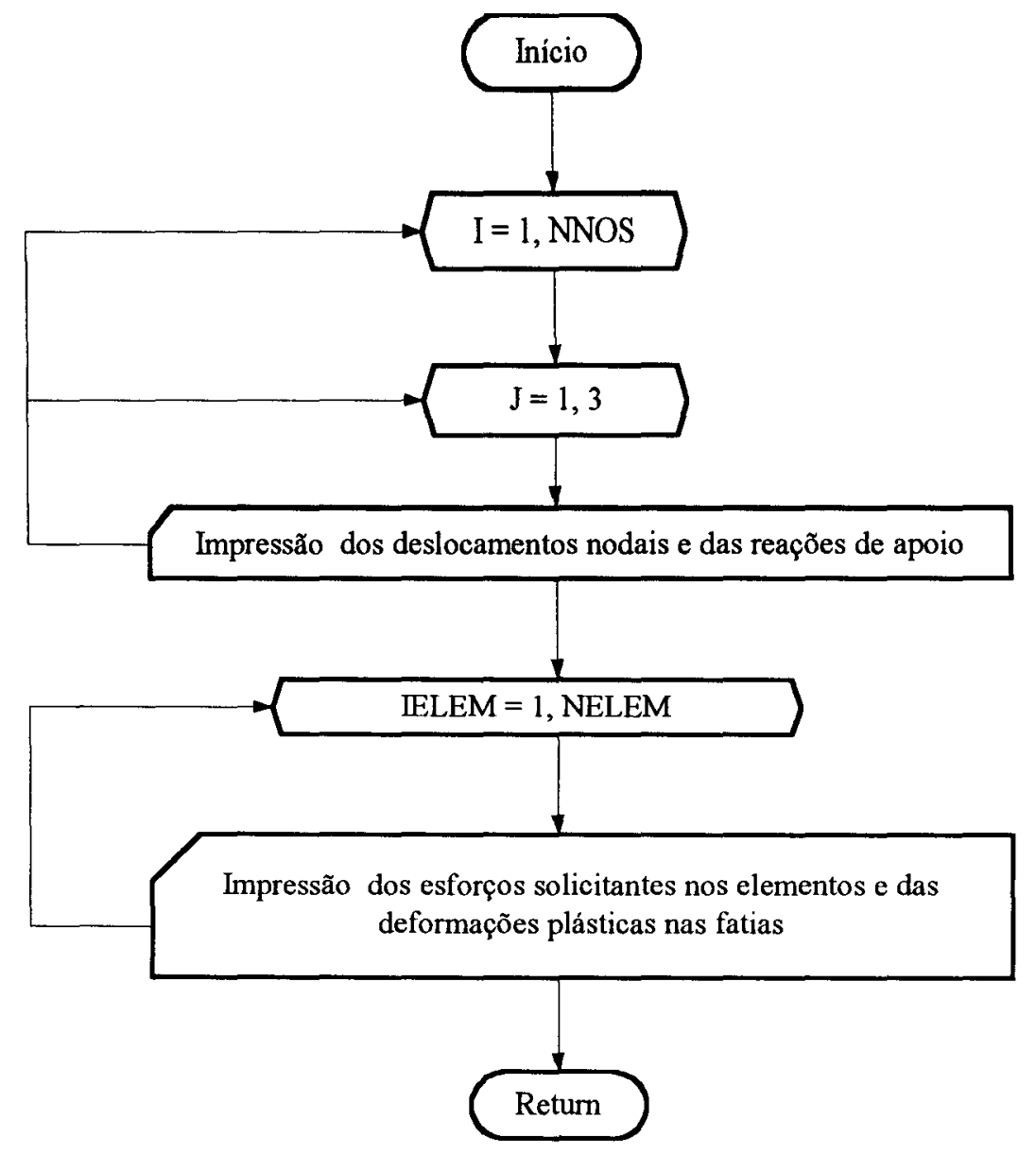




\subsection{Exemplos de concreto armado}

Aqui serão apresentados vários exemplos numéricos, onde será demonstrada a eficiência da formulação apresentada anteriormente, utilizandose o programa $\mathrm{PF}$, descrito no item anterior.

Os exemplos têm o objetivo de comparar os resultados obtidos do programa $\mathrm{PF}$ com valores experimentais e com aqueles obtidos por outros pesquisadores, avaliando-se assim a eficiência da formulação, assim como de comparar os resultados da análise não-linear aqui desenvolvida com aqueles obtidos por procedimentos aproximados prescritos pelas normas, tais como, $o$ método P- $\Delta$ e o método do amplificador de momentos, recomendado pelo ACI-318/89 e CEB-FIP (1978).

\subsubsection{Exemplo 1}

Trata-se de um pórtico de concreto armado testado experimentalmente por ERNST et al (1973). As colunas e as vigas têm as mesmas características geométricas. A Fig. 4.8 mostra o pórtico e a seção tranversal. O concreto tem $\mathrm{f}_{\mathrm{cm}}=4220 \mathrm{psi}, \varepsilon_{\mathrm{o}}=0,002$ e o aço tem $\mathrm{f}_{\mathrm{y}}=51200 \mathrm{psi} \mathrm{e}_{\mathrm{s}}=29000 \mathrm{ksi}$.
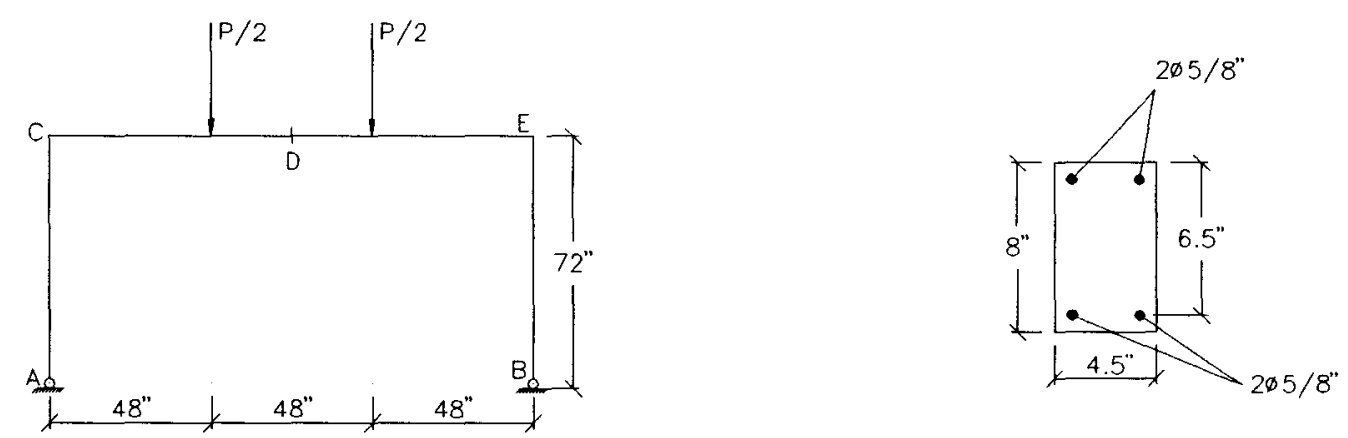

FIGURA 4.8 - Pórtico plano do exemplo 1 
A estrutura foi analisada discretizando-se cada barra com 8 elementos. A Tab. 4.1 mostra os resultados da análise quando se considera a resistência do concreto à tração e quando se despreza esta resistência.

TABELA 4.1 - Resultados da análise do pórtico do exemplo 1

\begin{tabular}{|c|c|c|c|}
\cline { 2 - 4 } \multicolumn{1}{c|}{} & $\begin{array}{c}\text { Análise } \\
\text { com tração }\end{array}$ & $\begin{array}{c}\text { Análise } \\
\text { sem tração }\end{array}$ & Experimental \\
\hline $\begin{array}{c}\text { Carga última } \\
\text { (Kips) }\end{array}$ & 18,4 & 14,8 & 15,9 \\
\hline $\begin{array}{c}\text { Deslocamento } \\
\text { último (in) }\end{array}$ & 0,85 & 0,79 & 1,8 \\
\hline
\end{tabular}

No modelo que não considera a resistência do concreto à tração a carga última difere da obtida experimentalmente em menos de $10 \%$, enquanto que no modelo que considera a resistência à tração esta diferença é de $16 \%$.

Com ambos os modelos os resultados podem ser considerados bons, embora nos pareça que o modelo que não considera a resistência à tração representa melhor o comportamento do concreto. Cabe ressaltar aqui que a resistência à tração do concreto não é unicamente uma função da sua resistência característica à compressão mas igualmente depende da maneira de preparação, lançamento e cura do concreto.

Os resultados da análise mostram uma estrutura mais rígida que a real nas proximidades do colapso, que pode ser explicado pelo fato do modelo utilizado não considerar as deformações por cisalhamento.

Com o objetivo de mostrar a influência da discretização longitudinal das barras, este mesmo pórtico foi analisado utilizando-se $2,4,8$ ou 20 elementos e os resultados praticamente não se alteraram, o que pode ser justificado pelo fato da estrutura ser simétrica, estar submetida só a cargas verticais e ter pouca não-linearidade geométrica. 


\subsubsection{Exemplo 2}

Trata-se de um pórtico de concreto armado testado experimentalmente por ERNST et al (1973). As seções transversais das vigas e colunas são diferentes. A Fig. 4.9 mostra o pórtico e as seções tranversais. O concreto tem $f_{c m}=5920$ psi, $\varepsilon_{o}=0,002$ e o aço tem $f_{y}=67000$ psi e $E_{s}=29000$ ksi.
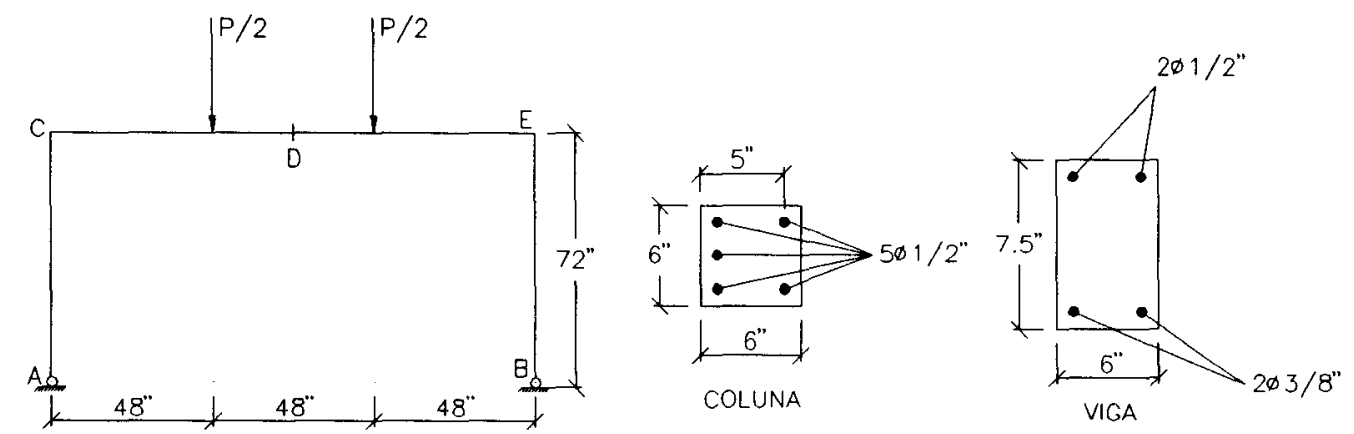

FIGURA 4.9 - Pórtico plano do exemplo 2

A estrutura foi analisada discretizando-se cada barra com 8 elementos. A Tab. 4.2 mostra os resultados da análise quando se considera a resistência do concreto à tração e quando se despreza esta resistência.

TABELA 4.2 - Resultados da análise do pórtico do exemplo 2

\begin{tabular}{|c|c|c|c|}
\cline { 2 - 4 } \multicolumn{1}{c|}{} & $\begin{array}{c}\text { Análise } \\
\text { com tração }\end{array}$ & $\begin{array}{c}\text { Análise } \\
\text { sem tração }\end{array}$ & Experimental \\
\hline $\begin{array}{c}\text { Carga última } \\
\text { (Kips) }\end{array}$ & 17,2 & 14,2 & 14,9 \\
\hline $\begin{array}{c}\text { Deslocamento } \\
\text { último (in) }\end{array}$ & 0,84 & 0,96 & 1,50 \\
\hline
\end{tabular}


Também neste exemplo, os resultados da Tab. 4.2 mostram que 0 modelo constitutivo que não considera a resistência à tração do concreto apresenta resultado de carga última mais próximo do experimental, porém os resultados da análise mostram uma estrutura mais rígida nas proximidades do colapso. Os resultados de carga última podem ser considerados muito bons (erro de $5 \%$ no modelo sem tração e $15 \%$ no modelo com tração)

\subsubsection{Exemplo 3}

Um pórtico plano de concreto armado formado por três barras com seções transversais retangulares conforme Fig. 4.10, discretizado em 10 elementos por barra, com as seguintes características dos materiais:

$$
\mathrm{f}_{\mathrm{cm}}=17,5 \mathrm{MPa} ; \varepsilon_{\mathrm{o}}=0,002 ; \mathrm{f}_{\mathrm{y}}=420 \mathrm{MPa} \text { e } \mathrm{E}_{\mathrm{s}}=210 \mathrm{GPa} \text {. }
$$

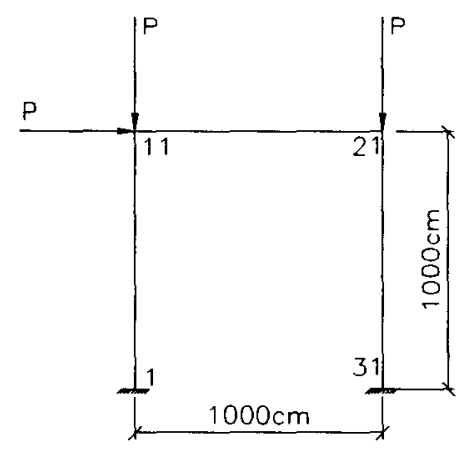

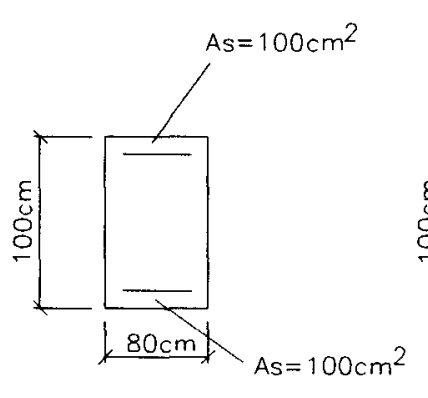

PILARES

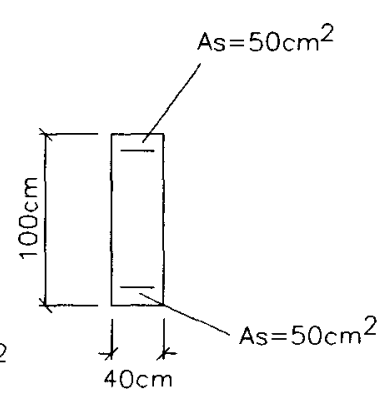

VIGA

FIGURA 4.10 - Pórtico plano do exemplo 3

A estrutura foi analisada considerando-se a resistência à tração do concreto e a carga última encontrada foi $P_{c r}=1400 \mathrm{KN}$ e, quando não se considerou resistência à tração do concreto a carga última foi $P_{c r}=1300 \mathrm{KN}$.

A mesma estrutura foi analisada por SOLER (1995), com a mesma discretização, sem consideração da resistência à tração do concreto e a carga última encontrada foi $P_{c r}=1375 \mathrm{KN}$. 
Neste exemplo, a consideração de resistência à tração do concreto alterou pouco $(8 \%)$ a carga última.

Para verificar a influência da discretização longitudinal das barras, as mesmas foram discretizadas em 2, 5, 10 ou 20 elementos por barra, sendo que as cargas últimas encontradas, quando não se considerou a resistência à tração do concreto, estão listadas na Tab. 4.3.

TABELA 4.3 - Influência da discretização longitudinal das barras

\begin{tabular}{|c|c|}
\hline $\begin{array}{c}\text { Elementos } \\
\text { por barra }\end{array}$ & $\begin{array}{c}\text { Carga última } \\
(\mathrm{KN})\end{array}$ \\
\hline 2 & 1900 \\
\hline 5 & 1800 \\
\hline 10 & 1300 \\
\hline 20 & 1300 \\
\hline
\end{tabular}

Neste exemplo fica evidente a necessidade de uma maior discretização das barras quando se tratar de estruturas com as duas não-linearidades. Considerando-se que este pórtico tem a viga horizontal fortemente submetida a uma flexão anti-simétrica, pode-se concluir que a discretização da barra em 10 elementos é suficiente para análise de pórticos usuais de edifícios de concreto armado.

Quanto à discretização da seção tranversal, observou-se que 10 fatias são suficientes para se obter bons resultados. Neste exemplo a seção foi discretizada em 10 e 20 fatias e o resultado praticamente não se alterou. 


\subsubsection{Exemplo 4}

Trata-se de um pórtico plano de concreto armado (Fig. 4.11) originalmente apresentado no artigo de CORRADI et al (1974). A mesma estrutura foi resolvida por outros autores como CAUVIN (1978) e CILONI (1993). A estrutura será discretizada em 96 elementos, sendo 8 elementos em cada viga e 4 elementos por coluna, como feito por CILONI. Os valores da propriedades físicas dos materiais adotados são:

$$
\mathrm{f}_{\mathrm{cm}}=20 \mathrm{MPa} ; \varepsilon_{\mathrm{o}}=0,002 ; \mathrm{f}_{\mathrm{y}}=500 \mathrm{MPa} \text { e } \mathrm{E}_{\mathrm{s}}=200 \mathrm{GPa} \text {. }
$$
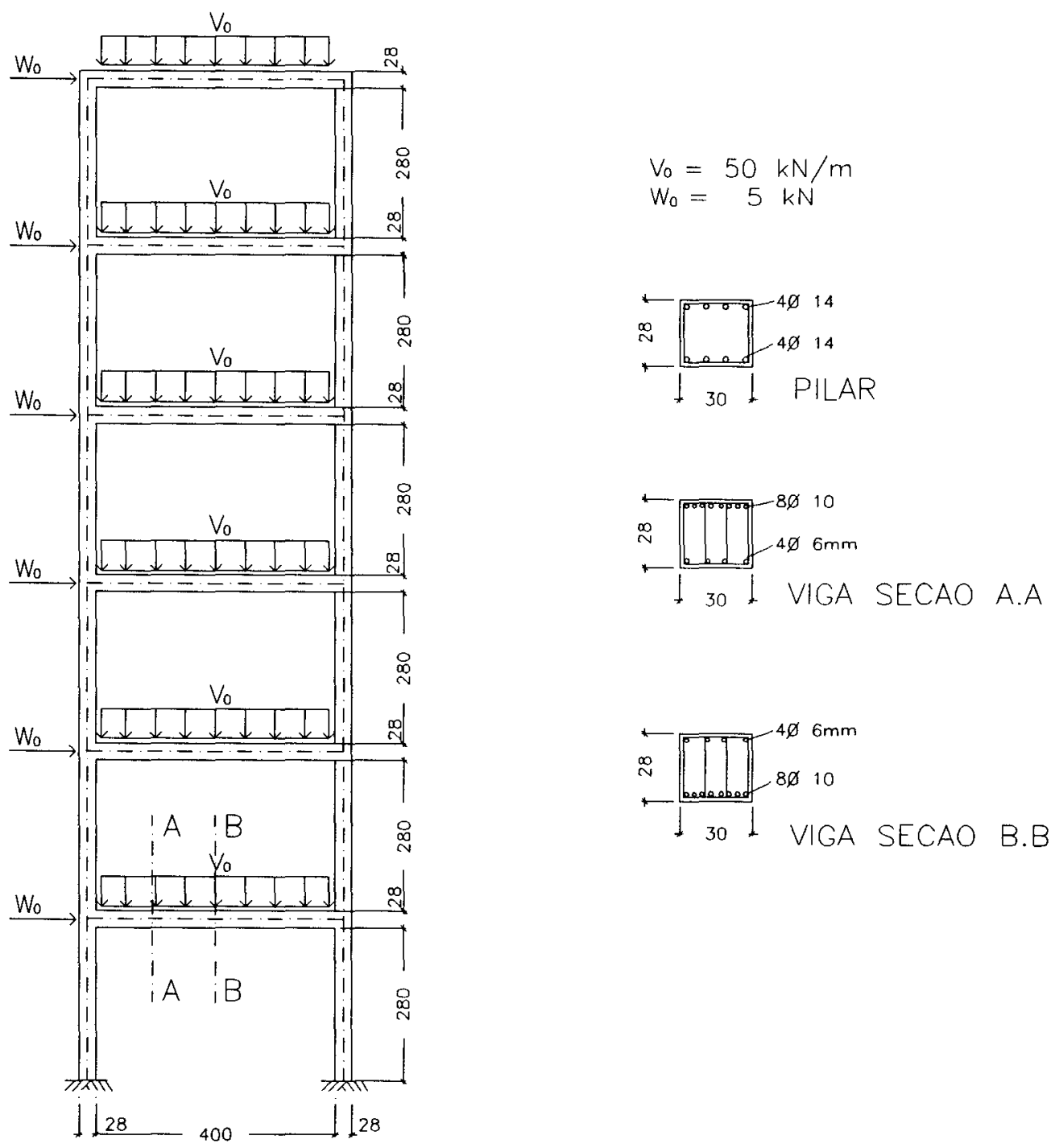

FIGURA 4.11 - Pórtico plano do exemplo 4 
A Tab. 4.4 apresenta os resultados comparativos entre os valores obtidos por CAUVIN (1978), por CILONI (1993) e com o programa PF desenvolvido neste trabalho. Na análise feita com o PF, considerou-se a resistência à tração do concreto, como fizeram CAUVIN e CILONI.

Para efeito de análise dos deslocamentos considerou-se a carga distribuída de $50 \mathrm{KN} / \mathrm{m}$.

TABELA 4.4 - Deslocamentos laterais do pórtico do exemplo 4 (em cm)

\begin{tabular}{|c|c|c|c|}
\hline Pavimento & CAUVIN & CILONI & PF \\
\hline 6 & 5,27 & 5,69 & 5,90 \\
\hline 5 & 4,88 & 5,18 & 5,44 \\
\hline 4 & 4,21 & 4,35 & 4,70 \\
\hline 3 & 3,24 & 3,27 & 3,62 \\
\hline 2 & 1,97 & 1,99 & 2,24 \\
\hline 1 & 0,68 & 0,71 & 0,77 \\
\hline
\end{tabular}

Os deslocamentos obtidos com o programa PF são um pouco maiores que os apresentados por CAUVIN e por CILONI, visto ser o modelo utilizado por eles mais rígido uma vez que eles consideram a contribuição do concreto intacto entre as fissuras ("tension stiffening"). Assim, pode-se considerar boa a concordância entre os resultados.

Uma segunda análise foi feita, considerando-se a carga uniformemente distribuída de $45 \mathrm{KN} / \mathrm{m}$ e os resultados são comparados com os obtidos por CILONI e por KRISHNAMOORTHY et al (1990).

A Tab. 4.5 mostra os valores dos momentos fletores nas diversas seções do pórtico (Fig. 4.12), obtidos por CILONI (programa PORANLI), 
KRISHNAMOORTHY (programas QPFRAM e CONFAP) e pelo autor (programa PF).

Observa-se uma boa concordância entre os resultados.

Cabe observar ainda, que para este carregamento ocorreu o escoamento das armaduras nas seções 33, 36, 39 e 42 e, uma subsequente redistribuição dos esforços, mostrando a eficiência do programa na região plástica.

TABELA 4.5 - Momentos fletores no pórtico do exemplo 4

\begin{tabular}{|c|c|c|c|c|}
\hline Seção & QPFRAM & CONFAP & PORANLI & PF \\
\hline 1 & $-48,98$ & $-48,96$ & $-48,808$ & $-48,44$ \\
\hline 2 & 34,40 & 34,40 & 33895 & 35,64 \\
\hline 3 & 60,25 & 60,25 & 58,701 & 61,02 \\
\hline 4 & $-38,53$ & $-38,53$ & $-37,713$ & $-39,33$ \\
\hline 5 & $-16,09$ & $-16,45$ & $-19,113$ & $-13,99$ \\
\hline 6 & 27,38 & 27,71 & 26,663 & 25,54 \\
\hline 7 & 38,61 & 39,08 & 38,734 & 38,03 \\
\hline 8 & $-35,66$ & $-35,85$ & $-34,556$ & $-35,16$ \\
\hline 9 & $-15,02$ & $-15,14$ & $-19,523$ & $-14,78$ \\
\hline 10 & 25,19 & 25,29 & 26,778 & 22,43 \\
\hline 11 & 46,58 & 46,70 & 47,827 & 48,44 \\
\hline 12 & $-39,82$ & $-39,99$ & $-40,560$ & $-41,16$ \\
\hline 13 & $-10,04$ & $-10,35$ & $-15,501$ & $-9,25$ \\
\hline 14 & 18,15 & 18,25 & 19,749 & 14,90 \\
\hline 15 & 46,82 & 46,96 & 48,583 & 50,85 \\
\hline 16 & $-42,98$ & $-43,28$ & $-43,358$ & $-45,46$ \\
\hline 17 & $-11,77$ & $-12,11$ & $-19,753$ & $-10,27$ \\
\hline 18 & 8,39 & 8,17 & 16,832 & 8,70 \\
\hline
\end{tabular}


TABELA 4.5 - Momentos fletores no pórtico do exemplo 4

\begin{tabular}{|l|l|l|l|l|}
\hline 19 & 46,49 & 46,49 & 48,895 & 52,43 \\
\hline 20 & 50,67 & 50,79 & 58,708 & 57,69 \\
\hline 21 & 24,42 & 25,22 & 25,776 & 19,55 \\
\hline 22 & $-26,66$ & $-27,38$ & $-26,826$ & $-25,88$ \\
\hline 23 & 36,51 & 36,67 & 29,547 & 36,34 \\
\hline 24 & $-49,44$ & $-49,35$ & $-54,572$ & $-54,80$ \\
\hline 25 & $-48,98$ & $-48,96$ & $-48,807$ & $-48,44$ \\
\hline 26 & 50,58 & 48,44 & 48,172 & 48,60 \\
\hline 27 & $-60,25$ & $-60,25$ & $-58,718$ & $-61,02$ \\
\hline 28 & $-50,49$ & $-50,85$ & $-53,020$ & $-49,62$ \\
\hline 29 & 40,66 & 38,81 & 37,297 & 39,50 \\
\hline 30 & $-77,14$ & $-77,61$ & $-76,461$ & $-77,36$ \\
\hline 31 & $-42,39$ & $-42,85$ & $-46,194$ & $-40,32$ \\
\hline 32 & 42,15 & 40,34 & 37,895 & 41,12 \\
\hline 33 & $-82,24$ & $-82,55$ & $-82,397$ & $-83,59$ \\
\hline 34 & $-35,24$ & $-35,64$ & $-42,276$ & $-31,67$ \\
\hline 35 & 43,54 & 41,74 & $-36,687$ & 41,21 \\
\hline 36 & $-86,64$ & $-86,96$ & $-89,161$ & $-92,01$ \\
\hline 37 & $-29,93$ & $-30,36$ & $-39,502$ & $-25,18$ \\
\hline 38 & 44,77 & 42,197 & 36,773 & 41,54 \\
\hline 39 & $-89,47$ & $-89,77$ & $-92,258$ & $-97,88$ \\
\hline 40 & $-32,81$ & $-33,38$ & $-42,610$ & $-28,25$ \\
\hline 41 & 44,47 & 42,62 & 37,475 & 41,88 \\
\hline 42 & $-87,19$ & $-87,46$ & $-88,255$ & $-94,03$ \\
\hline & & & & \\
\hline 38 & \\
\hline
\end{tabular}




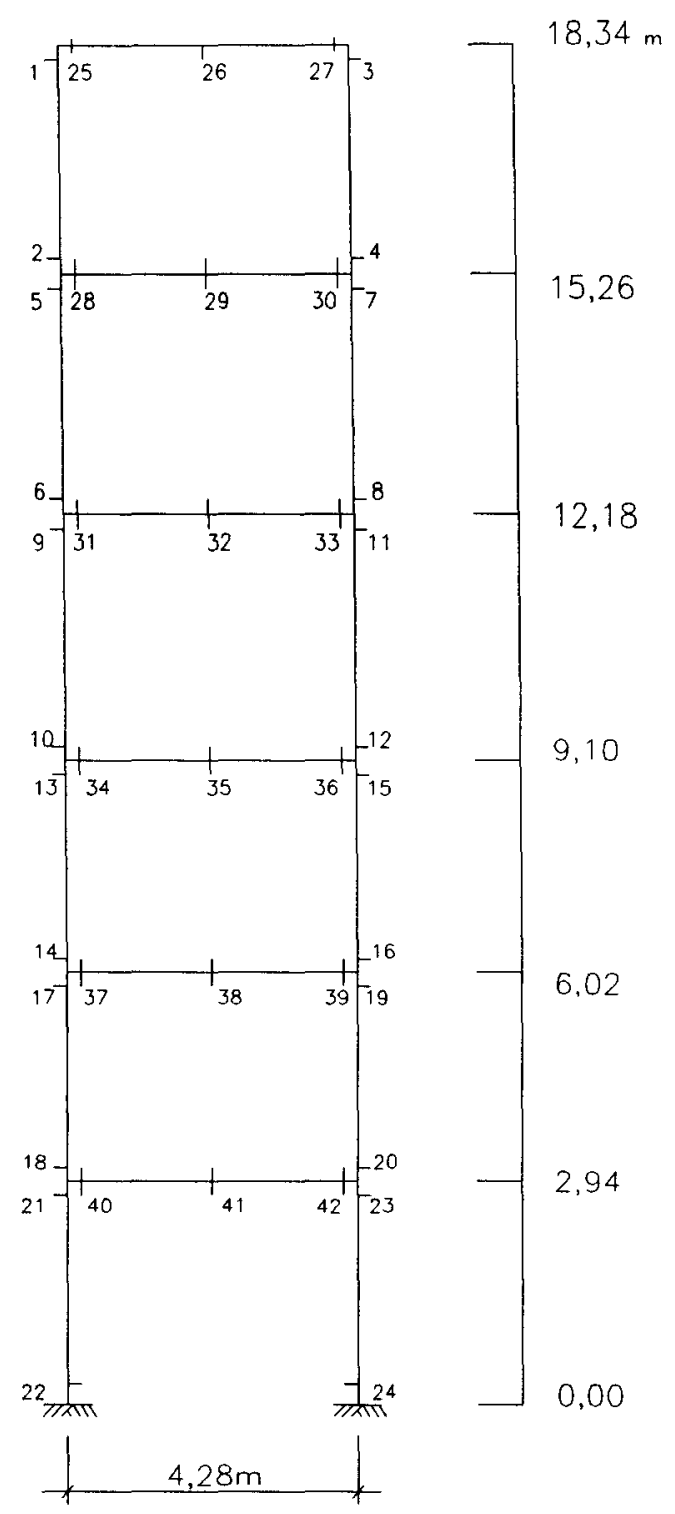

FIGURA 4.12 - Numeração das seções do pórtico do exemplo 4

Com o objetivo de avaliar os métodos simplificados de análise nãolinear prescritos pelas normas, o pórtico da Fig. 4.11 foi analisado utilizandose um programa de análise linear em $1^{\mathrm{a}}$ ordem e os deslocamentos e os esforços foram calculados, levando-se em consideração os efeitos não-lineares físicos e geométricos pelo método P- $\Delta$. Para avaliar os efeitos da nãolinearidade física do concreto armado, usou-se a rigidez da seção fissurada. 
Como vários pesquisadores têm recomendado diferentes valores para a rigidez da seção fissurada, aqui serão comparados os resultados obtidos quando se adotam estes diferentes valores de EI.

- Caso 1: Recomendação de VASCONCELOS \& FRANCO (1991)

- $\mathrm{EI}=0,50 \mathrm{E}_{\mathrm{c}} \mathrm{I}_{\mathrm{g}}$ para vigas

- $\mathrm{EI}=0,80 \mathrm{E}_{\mathrm{c}} \mathrm{I}_{\mathrm{g}}$ para colunas

- Caso 2: Recomendação de MACGREGOR \& HAGE (1977)

- $\mathrm{EI}=0,40 \mathrm{E}_{\mathrm{c}} \mathrm{I}_{\mathrm{g}}$ para vigas

- $\mathrm{EI}=0,80 \mathrm{E}_{\mathrm{c}} \mathrm{I}_{\mathrm{g}}$ para colunas

- Caso 3: Recomendação do ACI-318/89 (item 10.10.1)

- $\mathrm{EI}=0,50 \mathrm{E}_{\mathrm{c}} \mathrm{I}_{\mathrm{g}}$ para vigas

- $\mathrm{EI}=\left(0,20+1.2 \rho_{\mathrm{t}} \mathrm{E}_{\mathrm{s}} / \mathrm{E}_{\mathrm{c}}\right) \mathrm{E}_{\mathrm{c}} \mathrm{I}_{\mathrm{g}}=0,31 \mathrm{E}_{\mathrm{c}} \mathrm{I}_{\mathrm{g}}$ para colunas

- Caso 4: Recomendação da proposta de revisão do ACI-318/89, MACGREGOR (1993)

- $\mathrm{EI}=0,35 \mathrm{E}_{\mathrm{c}} \mathrm{I}_{\mathrm{g}}$ para vigas

- $\mathrm{EI}=0,70 \mathrm{E}_{\mathrm{c}} \mathrm{I}_{\mathrm{g}}$ para colunas

sendo $E_{c}=2897 \mathrm{KN} / \mathrm{cm}^{2}$ o módulo de elasticidade do concreto, $E_{s}=21000$ $\mathrm{KN} / \mathrm{cm}^{2}$ o módulo de elasticidade do aço, $\mathrm{I}_{\mathrm{g}} \mathrm{o}$ momento de inércia da seção plena da viga ou coluna e $\rho_{t}$ a relação entre a área da armadura $e$ a área da seção tranversal da coluna.

A comparação entre os deslocamentos obtidos via análise não-linear pelo método P- $\Delta$ iterativo e aqueles fornecidos pelo programa PF estão mostrados na Tab. 4.6 
TABELA 4.6 - Deslocamentos laterais do pórtico do exemplo 4, via método $\mathrm{P}-\Delta(\mathrm{em} \mathrm{cm})$

\begin{tabular}{|c|c|c|c|c|c|}
\hline Andar & Caso 1 & Caso 2 & Caso 3 & Caso 4 & $\begin{array}{c}\text { Programa } \\
\text { PF }\end{array}$ \\
\hline 1 & 0,76 & 0,82 & 1,32 & 0,95 & 0,77 \\
\hline 2 & 2,04 & 2,31 & 2,26 & 2,70 & 2,24 \\
\hline 3 & 3,21 & 3,71 & 4,88 & 4,32 & 3,62 \\
\hline 4 & 4,10 & 4,79 & 6,07 & 5,58 & 4,70 \\
\hline 5 & 4,70 & 5,26 & 6,85 & 6,43 & 5,44 \\
\hline 6 & 5,06 & 5,96 & 7,26 & 6,93 & 5,90 \\
\hline
\end{tabular}

Os resultados dos momentos fletores estão mostrados na Tab. 4.7.

TABELA 4.7-Momentos fletores no pórtico do exemplo 4 via método P- $\Delta$ (em KN.m)

\begin{tabular}{|c|c|c|c|c|c|}
\hline Seção & Caso 1 & Caso 2 & Caso 3 & Caso 4 & $\begin{array}{c}\text { Programa } \\
\text { PF }\end{array}$ \\
\hline 21 & 15,07 & 19,31 & 4,60 & 19,25 & 23,69 \\
\hline 22 & $-25,96$ & $-26,54$ & $-20,23$ & $-27,21$ & $-26,18$ \\
\hline 23 & 39,00 & 35,75 & 42,82 & 35,79 & 38,38 \\
\hline 24 & $-53,04$ & $-54,12$ & $-43,96$ & $-54,78$ & $-58,56$ \\
\hline 40 & $-27,85$ & $-31,21$ & $-12,84$ & $-30,54$ & $-34,26$ \\
\hline 42 & $-97,94$ & $-96,73$ & $-98,28$ & $-97,40$ & $-100,85$ \\
\hline
\end{tabular}

Das Tab. 4.6 e 4.7 observa-se que nos casos 1,2 e 4 a análise nãolinear pelo método P- $\Delta$ iterativo leva a resultados com uma boa concordância com aqueles obtidos pela análise não-linear (programa PF), principalmente quando se usa os valores de EI recomendados por MACGREGOR \& HAGE 
(1977). Observa-se ainda, que os valores de EI recomendados pelo ACI318/89 (Item 10.10.1) são muito baixos, levando a uma superestimação dos deslocamentos e a uma distribuição de momentos muito diferente da obtida na análise não-linear (programa PF). Já os valores recomendados na proposta de revisão do ACl-318/89 levam a resultados compatíveis de momento de $2^{\underline{a}}$ ordem e superestimam os deslocamentos.

A Tab. 4.8 compara os valores dos momentos obtidos através da análise não-linear via programa $\mathrm{PF}$, com aqueles fornecidos pelo método do amplificador de momentos de $1^{\underline{a}}$ ordem, recomendado pela proposta de revisão do ACI-318/89, conforme MACGREGOR (1993). Os momentos de $1^{\mathrm{a}}$ ordem foram obtidos adotando-se $\mathrm{EI}_{\text {viga }}=0,35 \mathrm{E}_{\mathrm{c}} \mathrm{I}_{\mathrm{g}}$ e EI $\mathrm{I}_{\text {coluna }}=0,70 \mathrm{E}_{\mathrm{c}} \mathrm{I}_{\mathrm{g}}$.

TABELA 4.8 - Momentos fletores no pórtico do exemplo 4

\begin{tabular}{|c|c|c|}
\hline Seção & $\begin{array}{c}\text { Método do } \\
\text { amplificador }\end{array}$ & $\begin{array}{c}\text { Programa } \\
\text { PF }\end{array}$ \\
\hline 21 & 18,50 & 23,69 \\
\hline 22 & $-26,59$ & $-26,18$ \\
\hline 23 & 36,56 & 38,38 \\
\hline 24 & $-54,17$ & $-58,56$ \\
\hline 40 & $-35,40$ & $-34,26$ \\
\hline 42 & $-92,54$ & $-100,85$ \\
\hline
\end{tabular}

Observa-se uma boa concordância entre os valores obtidos pelo método do amplificador de momentos, exceto para a seção 21 , onde o momento é subestimado em $27 \%$. O parâmetro de estabilidade "Q" para o $1^{\circ}$ pavimento é igual a 0,107 .

Esta estrutura teve seu carregamento incrementado até atingir a carga última, que ocorreu com um fator de carregamento de 1,3 com um deslocamento máximo no topo de $9,94 \mathrm{~cm}$, o que mostra que mesmo para um 
nível de carga relativamente próximo do colapso, os métodos aproximados apresentam bons resultados.

Com o objetivo de mostrar a potencialidade do programa desenvolvido, a mesma estrutura sob ação do mesmo carregamento foi analisada considerando-se um recalque vertical de $2 \mathrm{~cm}$ no apoio da direita (seção 24) e o fator de carregamento último foi de 1,2 com deslocamento horizontal no topo de $16,86 \mathrm{~cm}$.

\subsubsection{Exemplo 5}

Trata-se de um pórtico plano de um edifício de 8 andares (Fig. 4.13), que foi analisado por CAUVIN (1978) e CILONI (1993). As propriedades dos materiais são:

$$
\begin{aligned}
& f_{c m}=16 \mathrm{MPa} \\
& f_{y}=400 \mathrm{MPa} \\
& E s=210 \mathrm{GPa} \\
& \varepsilon_{\mathrm{c}}=0,002
\end{aligned}
$$

As condições de carregamento estão representadas nas Fig. 4.14 e 4.15, com combinações para Estado Limite Último. Para que os resultados pudessem ser comparados com os de CILONI, utilizou-se a mesma discretização feita por ele, ou seja, cada viga dividida em 24 elementos de 60 $\mathrm{cm}$ e cada coluna dividida em 3 elementos de $120 \mathrm{~cm}$, perfazendo-se um total de 288 elementos e 268 nós. Considerou-se a resistência do concreto à tração. 

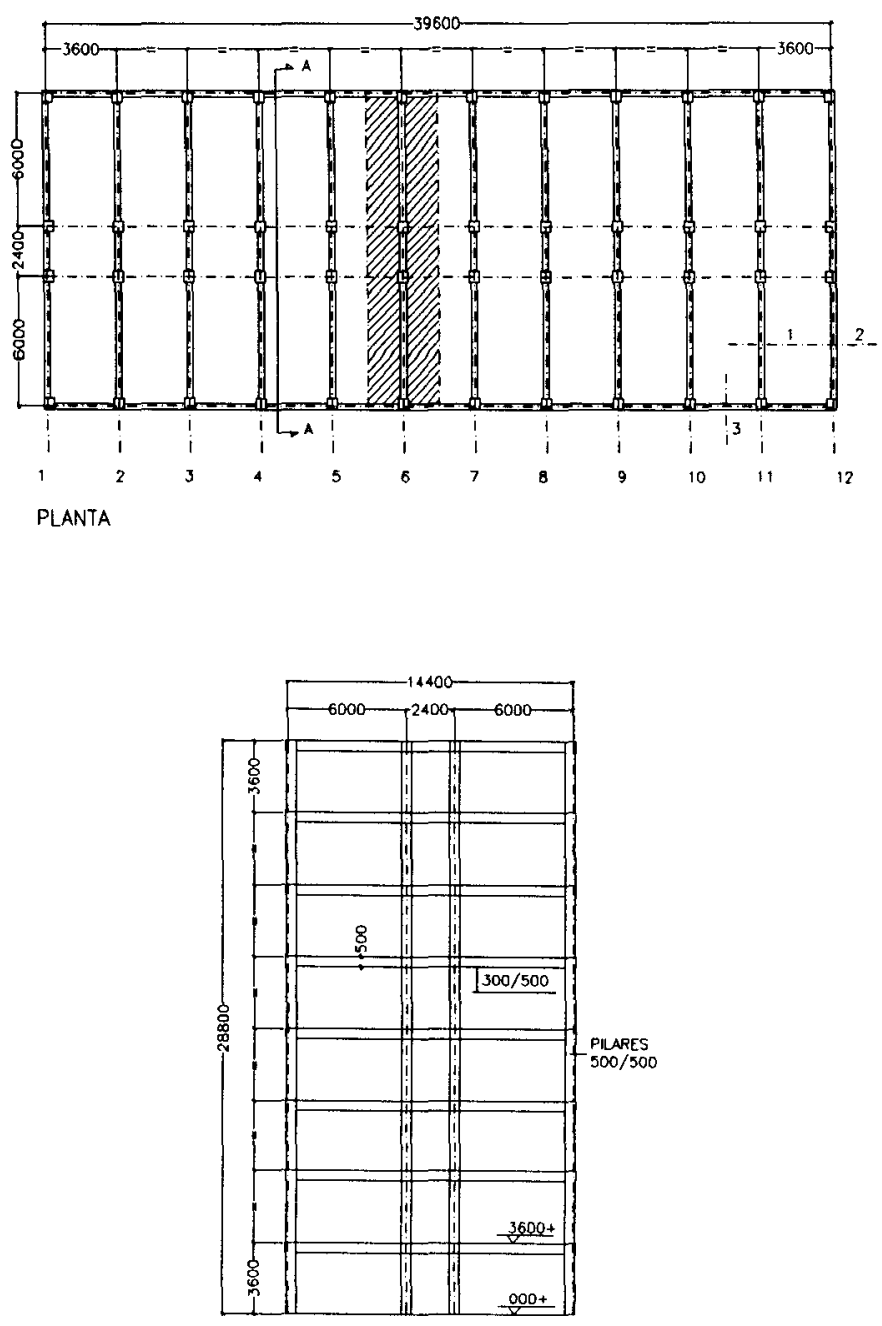

SECAO AA
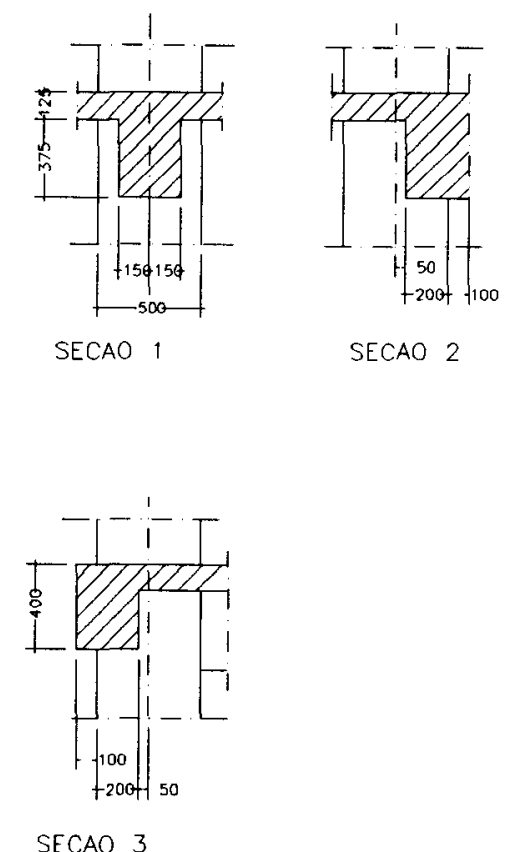

FIGURA 4.13 - Estrutura do edifício do exemplo 5 
- cargas acidentaIs em todos OS VĀOS.

- estado limite Último.

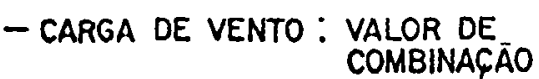

\begin{tabular}{|l|l|l|l|}
\hline \multicolumn{4}{|c|}{ CARGAS PERMANENTES } \\
\hline$q_{d}$ & 22.68 & $F_{d}$ & 43.20 \\
\hline$F_{\text {Cd }}$ & 25.92 & & \\
\hline CARGAS ACIOENTAIS & \\
\hline$q_{\text {fd }}$ & 19.93 & $q_{\text {Rd }}$ & 9.97 \\
\hline$q_{\text {sd }}$ & 2.492 & $q_{\mathrm{wd}}$ & 4.98 \\
\hline
\end{tabular}

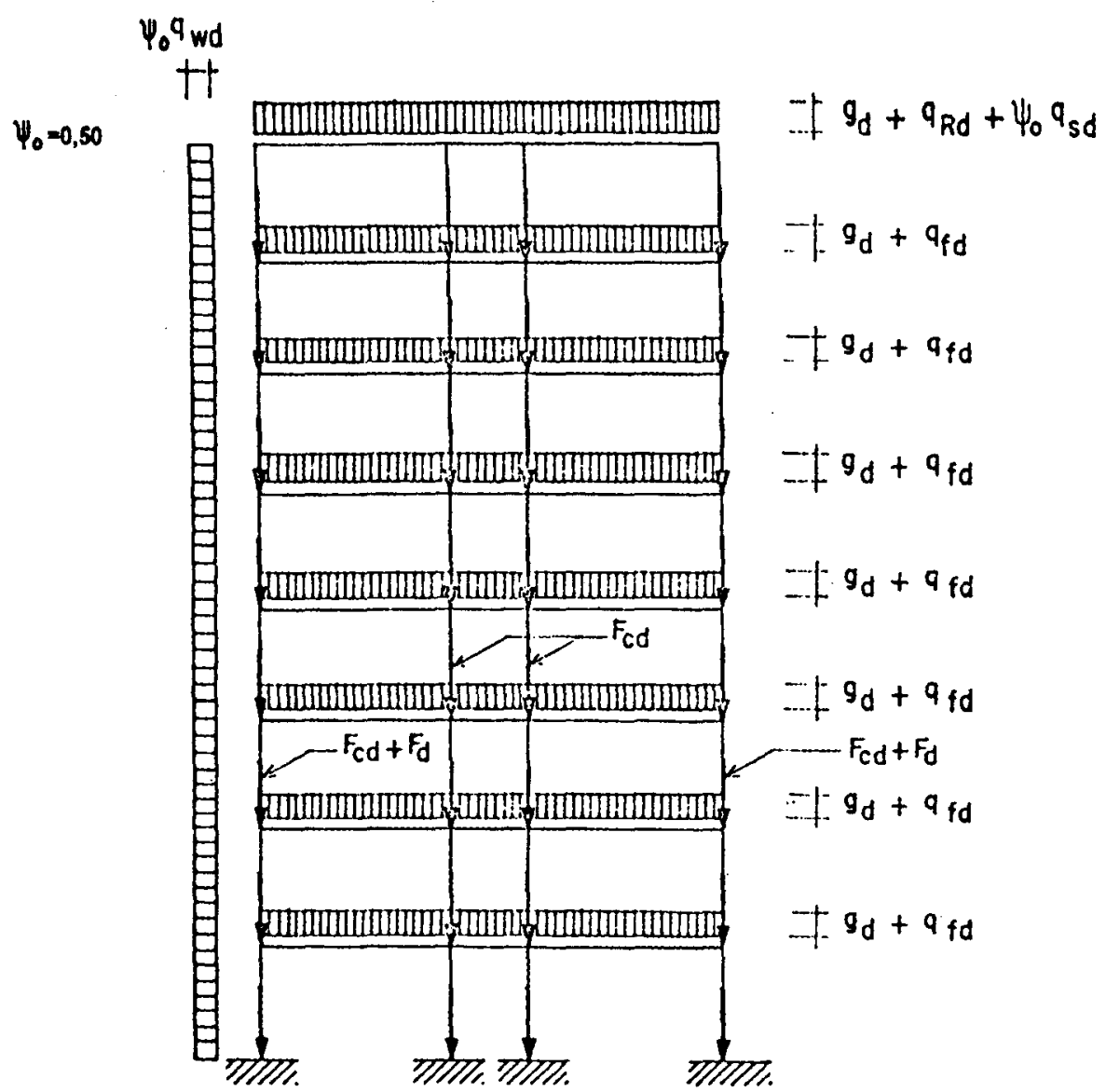

FIGURA 4.14 - Pórtico plano com carregamento 1 
- carga acioental em todos os VÃOS.

- eSTAdO LIMITE ÚlTIMO

- carga de Vento: valor básico

\begin{tabular}{|c|c|c|c|}
\hline \multicolumn{4}{|c|}{ CARGAS PERMANENTES } \\
\hline$g_{d}$ & 22.68 & $F_{d}$ & 43.20 \\
\hline$F_{c d}$ & 25.92 & & \\
\hline CARGAS ACIDENTAIS \\
\hline$q_{f d}$ & 19.93 & $q_{\text {Rd }}$ & 9.97 \\
\hline$q_{s d}$ & 2.462 & $q_{w d}$ & 4.98 \\
\hline
\end{tabular}

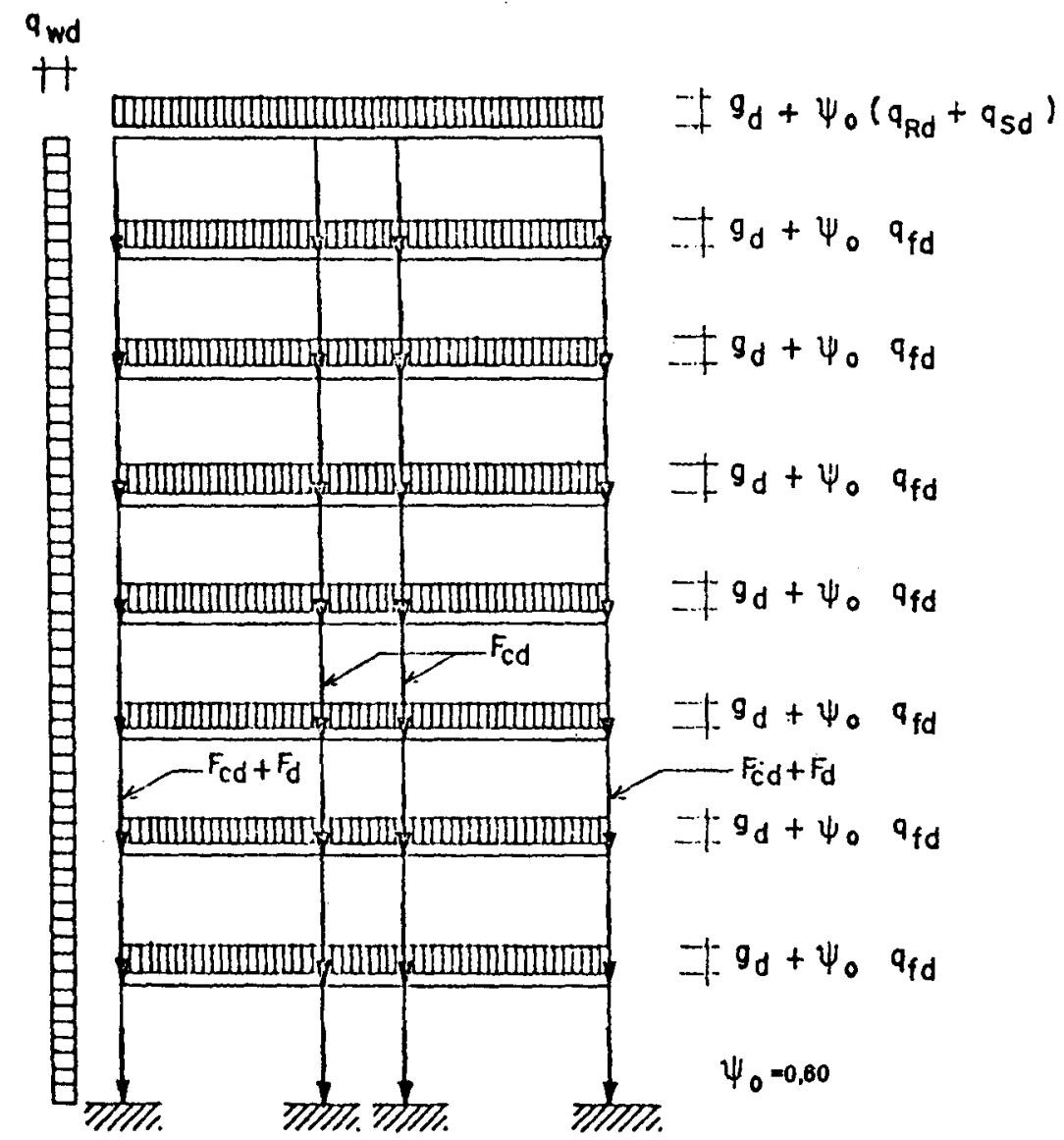

FIGURA 4.15 - Pórtico plano com carregamento 2

Na Fig. 4.16 estão representadas as armaduras das vigas e colunas. A estrutura foi discretizada em 3 tipos de elemento: um correspondente às colunas (seção $\mathrm{AA}$ ) e dois às vigas (seções $\mathrm{BB}$ e $\mathrm{CC}$ ). As vigas na seção $\mathrm{BB}$ foram consideradas como viga $T$ com largura da mesa igual a $130 \mathrm{~cm}$. 


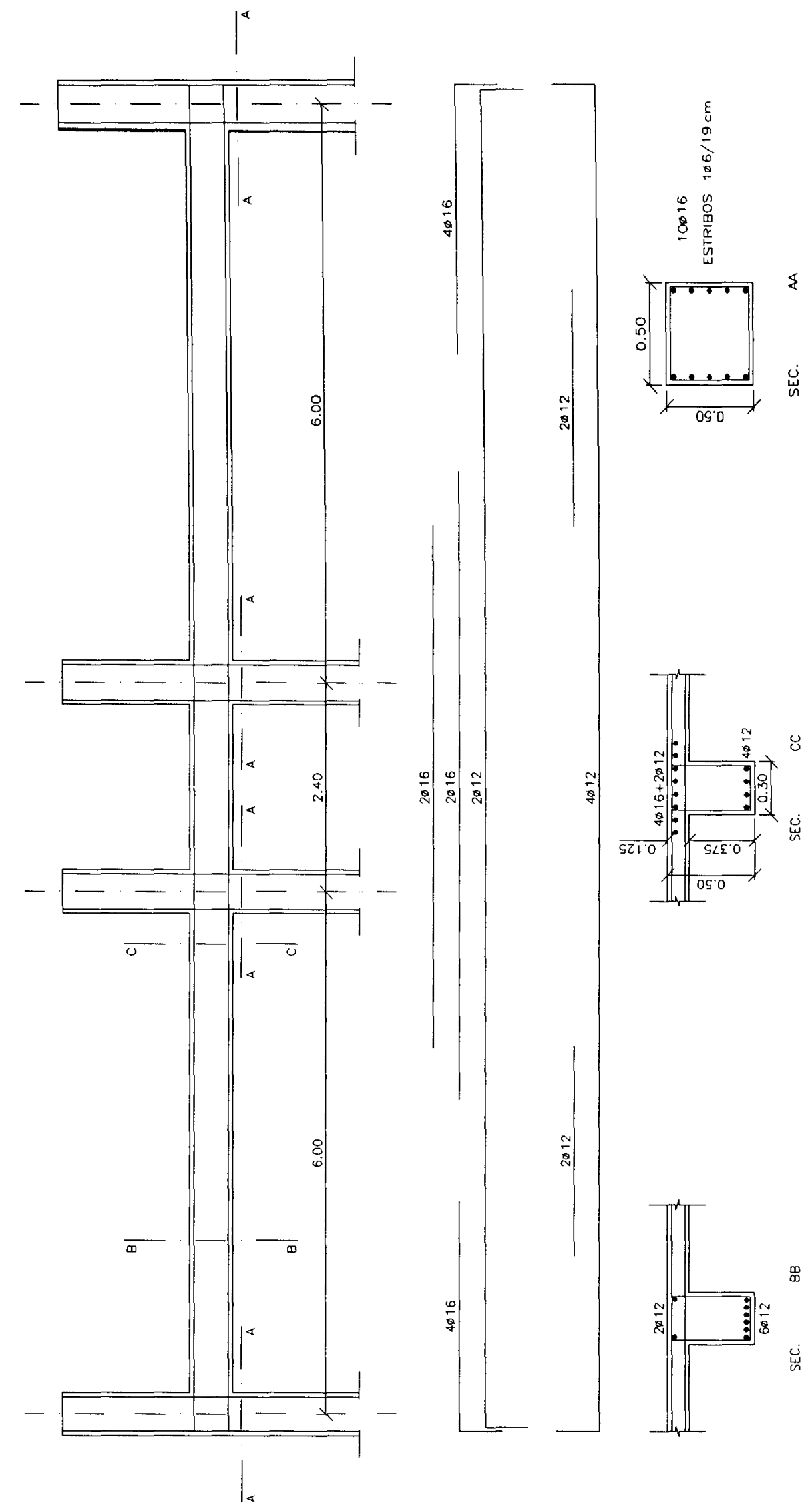

FIGURA 4.16 - Armadura do pórtico plano do Exemplo 5 
A Tab. 4.9 traz uma comparação entre os deslocamentos obtidos através do programa PF e aqueles fornecidos por CILONI (1993) e por CAUVIN (1978), para os carregamentos 1 e 2.

TABELA 4.9 - Deslocamentos laterais do pórtico do exemplo $5(\mathrm{em} \mathrm{cm})$

\begin{tabular}{|c|c|c|c|c|c|c|}
\hline \multirow{2}{*}{ Pavimento } & \multicolumn{3}{|c|}{ Carregamento 1 } & \multicolumn{3}{c|}{ Carregamento 2 } \\
\cline { 2 - 7 } & CAUVIN & CILONI & PF & CAUVIN & CILONI & PF \\
\hline 8 & 1,29 & 1,36 & 1,53 & 2,28 & 2,88 & 2,78 \\
\hline 7 & 1,23 & 1,23 & 1,44 & 2,16 & 2,73 & 2,65 \\
\hline 6 & 1,14 & 1,18 & 1,33 & 2,01 & 2,54 & 2,46 \\
\hline 5 & 1,01 & 1,03 & 1,17 & 1,76 & 2,27 & 2,19 \\
\hline 4 & 0,85 & 0,85 & 0,97 & 1,49 & 1,89 & 1,84 \\
\hline 3 & 0,64 & 0,63 & 0,73 & 1,10 & 1,39 & 1,40 \\
\hline 2 & 0,40 & 0,38 & 0,45 & 0,69 & 0,84 & 0,88 \\
\hline 1 & 0,15 & 0,14 & 0,16 & 0,26 & 0,30 & 0,33 \\
\hline
\end{tabular}

Os deslocamentos obtidos apresentam uma boa aproximação com aqueles obtidos por CILONI. Alguma discrepância se justifica pelo fato dos modelos utilizados serem diferentes, tanto no aspecto geométrico quanto no aspecto das relações constitutivas. No aspecto geométrico, a formulação aqui utilizada é mais precisa, já o modelo constitutivo de CILONI e de CAUVIN representa melhor o comportamento do concreto uma vez que ele considera a contribuição do concreto intacto entre as fisssuras ("tension stiffening").

A Tab. 4.10 traz uma comparação entre os momentos fletores em duas seções mais solicitadas da viga do $1^{\circ}$ pavimento obtidos através do programa PF e aqueles fornecidos por CILONI (1993) e por CAUVIN (1978), para os carregamentos 1 e 2 . CILONI e CAUVIN não apresentam resultados de esforços em colunas. 
TABELA 4.10 - Momentos fletores na viga do $1^{0}$ pavimento do pórtico do exemplo 5

\begin{tabular}{|c|c|c|c|c|c|c|}
\hline \multirow{2}{*}{ Seção } & \multicolumn{3}{|c|}{ Carregamento 1 } & \multicolumn{3}{c|}{ Carregamento 2 } \\
\cline { 2 - 7 } & CAUVIN & CILONI & PF & CAUVIN & CILONI & PF \\
\hline 9 & $-132,34$ & $-144,06$ & $-138,52$ & $-130,61$ & $-137,34$ & $-127,96$ \\
\hline 17 & $-133,39$ & $-143,07$ & $-138,97$ & $-132,49$ & $-136,75$ & $-130,48$ \\
\hline
\end{tabular}

Pode-se observar uma boa correlação entre estes momentos fletores.

As Tab. 4.11 e 4.12 mostram os valores dos deslocamentos laterais do topo do pórtico para os incrementos nos carregamentos 1 e 2 até a estrutura atingir as cargas últimas, o que ocorreu com fatores de carga iguais a 2,05 e 2,02 respectivamente.

Para o carregamento 1, o primeiro escoamento de armadura ocorreu com um fator de carga igual a 1,65 , nas seções 17,22 e 30 ; e para o carregamento 2 com um fator de carga igual a 1,60, na seção 30 (Fig. 4.17)

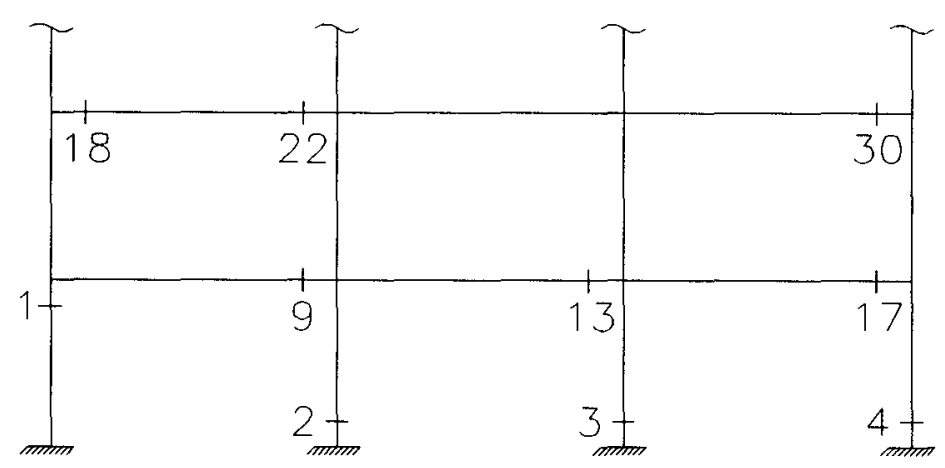

FIGURA 4.17 - Numeração das seções 
TABELA 4.11 - Deslocamentos laterais do topo do pórtico do exemplo 5 para o carregamento 1

\begin{tabular}{|c|c|}
\hline $\begin{array}{c}\text { Fator } \\
\text { de } \\
\text { carga }\end{array}$ & $\begin{array}{c}\text { Deslocamento } \\
\text { lateral do topo } \\
(\mathrm{cm})\end{array}$ \\
\hline 0,20 & 0,21 \\
\hline 0,40 & 0,45 \\
\hline 0,60 & 0,75 \\
\hline 0,80 & 1,12 \\
\hline 1,00 & 1,53 \\
\hline 1,20 & 1,95 \\
\hline 1,40 & 2,39 \\
\hline 1,60 & 2,87 \\
\hline 1,80 & 3,61 \\
\hline 1,90 & 4,19 \\
\hline 1,95 & 4,55 \\
\hline 2,00 & 4,97 \\
\hline 2,05 & colapso \\
\hline
\end{tabular}

TABELA 4.12- Deslocamentos laterais do topo do pórtico do exemplo 5 para o carregamento 2

\begin{tabular}{|c|c|}
\hline $\begin{array}{c}\text { Fator } \\
\text { de } \\
\text { carga }\end{array}$ & $\begin{array}{c}\text { Deslocamento } \\
\text { lateral do topo } \\
(\mathrm{cm})\end{array}$ \\
\hline 0,20 & 0,41 \\
\hline 0,40 & 0,86 \\
\hline 0,60 & 1,40 \\
\hline 0,80 & 2,04 \\
\hline
\end{tabular}


TABELA 4.12- Deslocamentos laterais do topo do pórtico do exemplo 5 para 0 carregamento 2

\begin{tabular}{|c|c|}
\hline 1,00 & 2,78 \\
\hline 1,20 & 3,60 \\
\hline 1,40 & 4,50 \\
\hline 1,60 & 5,47 \\
\hline 1,80 & 6,57 \\
\hline 1,90 & 7,31 \\
\hline 2,00 & 8,10 \\
\hline 2,02 & colapso \\
\hline
\end{tabular}

As Fig. 4.18 e 4.19 apresentam as curvas carga $\mathrm{x}$ deslocamento do pórtico para os carregamentos 1 e 2 respectivamente.

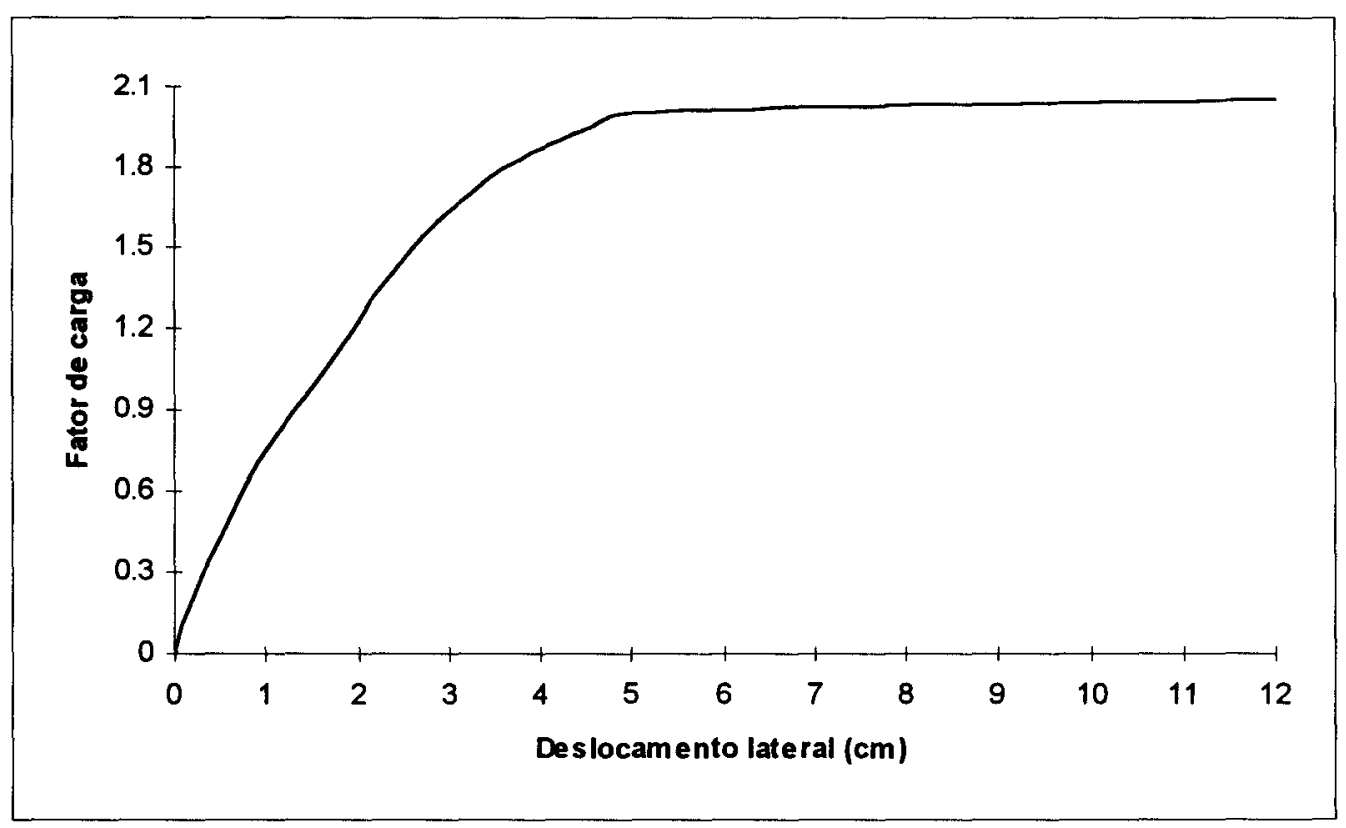

FIGURA 4.18 - Curva carga $\mathrm{x}$ deslocamento para o carregamento 1 


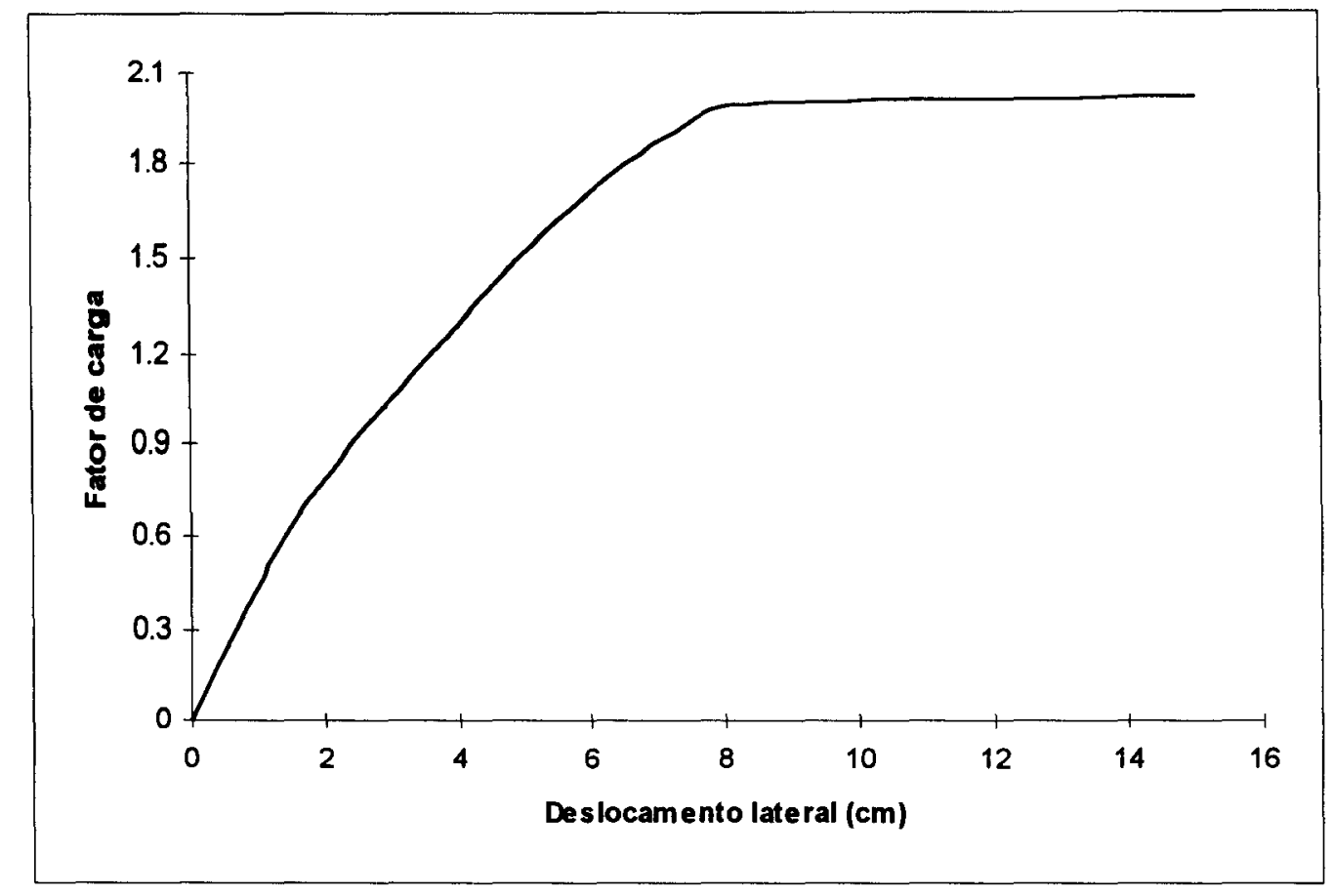

FIGURA 4.19- Curva carga $\mathrm{x}$ deslocamento para o carregamento 2

A mesma estrutura foi analisada via programa PF considerando-se um recalque vertical de $2 \mathrm{~cm}$ no apoio 3 , e os valores dos deslocamentos do topo do pórtico para os carregamentos 1 e 2 estão mostrados nas Tab. 4.13 e 4.14 respectivamente.

TABELA 4.13 - Deslocamentos laterais do topo do pórtico do exemplo 5 para o carregamento 1 , com recalque

\begin{tabular}{|c|c|}
\hline $\begin{array}{c}\text { Fator } \\
\text { de } \\
\text { carga }\end{array}$ & $\begin{array}{c}\text { Deslocamento } \\
\text { lateral do topo } \\
\text { (cm) }\end{array}$ \\
\hline 0.10 & 0.54 \\
\hline 0.20 & 1.10 \\
\hline 0.30 & 1.73 \\
\hline 0.40 & 2.42 \\
\hline 0.50 & 3.16 \\
\hline 0.60 & 3.95 \\
\hline
\end{tabular}


TABELA 4.13 - Deslocamentos laterais do topo do pórtico do exemplo 5 para o carregamento 1 , com recalque

\begin{tabular}{|l|l|}
\hline 0.70 & 4.77 \\
\hline 0.80 & 5.63 \\
\hline 0.90 & 6.58 \\
\hline 0.95 & colapso \\
\hline
\end{tabular}

TABELA 4.14 - Deslocamentos laterais do topo do pórtico do exemplo 5 para o carregamento 2, com recalque

\begin{tabular}{|c|c|}
\hline $\begin{array}{c}\text { Fator } \\
\text { de } \\
\text { carga }\end{array}$ & $\begin{array}{c}\text { Deslocamento } \\
\text { lateral do topo } \\
(\mathrm{cm})\end{array}$ \\
\hline 0,10 & 0.64 \\
\hline 0,20 & 1.33 \\
\hline 0,30 & 2.11 \\
\hline 0.40 & 2.98 \\
\hline 0,50 & 3.90 \\
\hline 0,60 & 4.87 \\
\hline 0,70 & 5.89 \\
\hline 0,80 & 6.95 \\
\hline 0,90 & 8.11 \\
\hline 1,00 & 9.44 \\
\hline 1,05 & colapso \\
\hline
\end{tabular}

Como era esperado, considerando-se o recalque os fatores de carga última para os carregamentos 1 e 2 se reduziram para 0,95 e 1,05, respectivamente. $O$ primeiro escoamento de armadura para o carregamento 1 
ocorreu na seção 17 , para um fator de carga igual a 0,70 e, para 0 carregamento 2 ocorreu na seção 13 para um fator de carga igual a 0,80 .

As Fig. 4.20 e 4.21 apresentam as curvas carga $\mathrm{x}$ deslocamento do pórtico para os carregamentos 1 e 2 respectivamente, considerando-se o recalque.

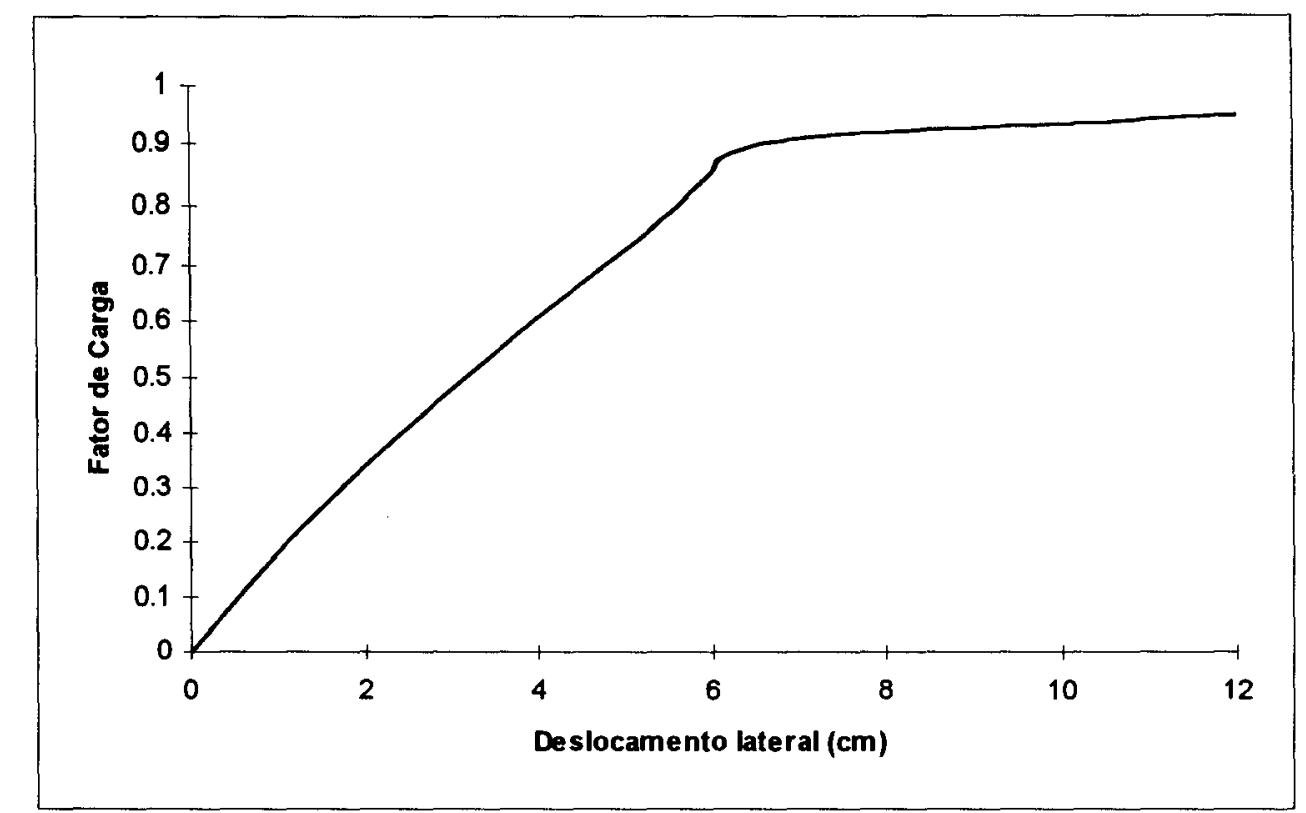

FIGURA 4.20 - Curva carga $x$ deslocamento para o carregamento $1 \mathrm{com}$ recalque

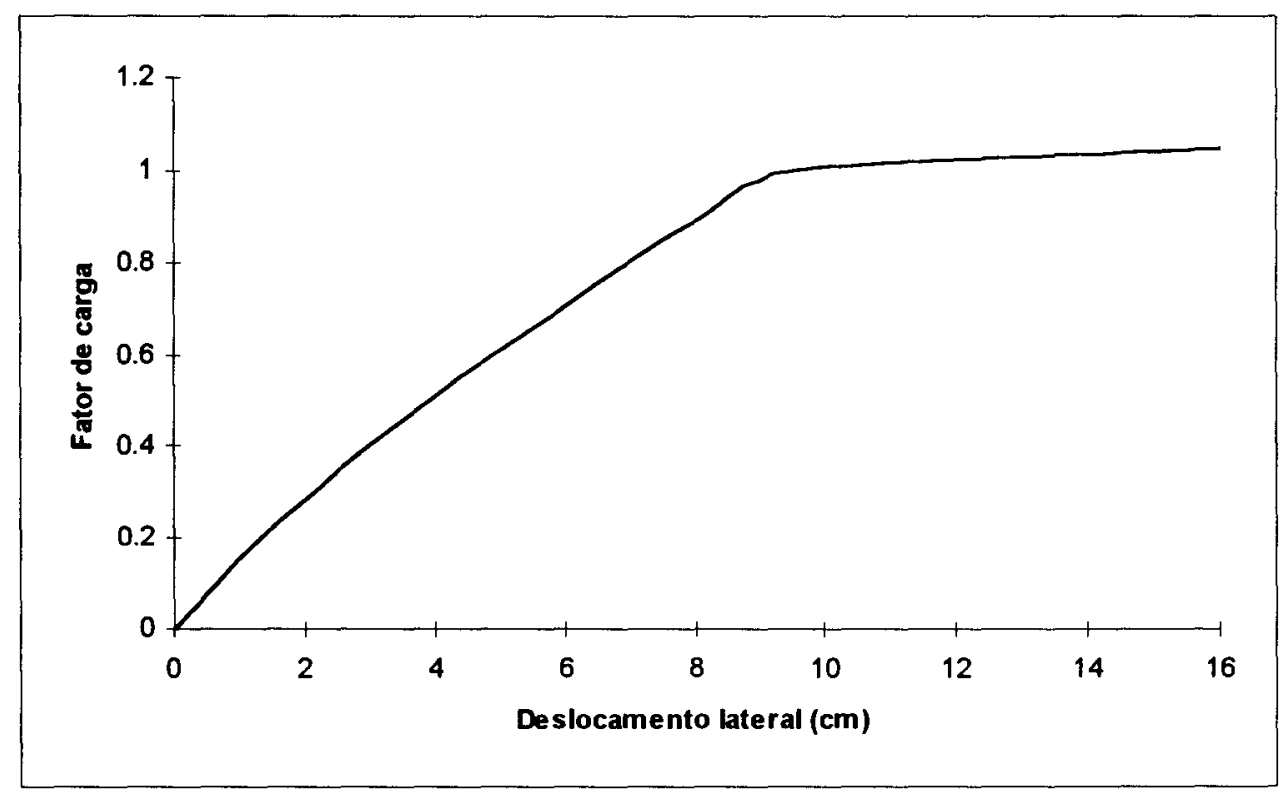

FIGURA 4.21 - Curva carga $\mathrm{x}$ deslocamento para o carregamento 2 com recalque 
Com o objetivo de se avaliar os métodos aproximados, a estrutura foi analisada pelo método P- $\Delta$ iterativo, considerando-se a inércia da sessão fissurada através da adoção dos seguintes valores de EI para vigas e colunas:

- Caso 1: Recomendações de VASCONCELOS \& FRANCO (1991)

- $\mathrm{EI}=0,50 \mathrm{E}_{\mathrm{c}} \mathrm{I}_{\mathrm{g}}$ para vigas

- $\mathrm{EI}=0,80 \mathrm{E}_{\mathrm{c}} \mathrm{I}_{\mathrm{g}}$ para colunas

- Caso 2: Recomendações de MACGREGOR \& HAGE (1977)

- $\mathrm{EI}=0,40 \mathrm{E}_{\mathrm{c}} \mathrm{I}_{\mathrm{g}}$ para vigas

- $\mathrm{EI}=0,80 \mathrm{E}_{\mathrm{c}} \mathrm{I}_{\mathrm{g}}$ para colunas

- Caso 3: Recomendações do ACI-318/89 (item 10.10.1)

- $\mathrm{EI}=0,50 \mathrm{E}_{\mathrm{c}} \mathrm{I}_{\mathrm{g}}$ para vigas

- $E I=\left(0,20+1.2 \rho_{t} E_{s} / E_{c}\right) E_{c} I_{g}=0,28 E_{c} I_{g}$ para colunas

- Caso 4: Recomendação da proposta de revisão do ACI-318/89, MACGREGOR (1993)

- $\mathrm{EI}=0,35 \mathrm{E}_{\mathrm{c}} \mathrm{I}_{\mathrm{g}}$ para vigas

- $\mathrm{EI}=0,70 \mathrm{E}_{\mathrm{c}} \mathrm{I}_{\mathrm{g}}$ para colunas

sendo $E_{c}=2639 \mathrm{KN} / \mathrm{cm}^{2}$.

As Tab. 4.15 e 4.16 trazem uma comparação entre os deslocamentos obtidos via método $\mathrm{P}-\Delta \mathrm{e}$ aqueles fornecidos pelo programa $\mathrm{PF}$. 
TABELA 4.15 - Deslocamentos laterais do pórtico para o carregamento 1, via método $\mathrm{P}-\Delta$

\begin{tabular}{|c|c|c|c|c|c|}
\hline Andar & Caso 1 & Caso 2 & Caso 3 & Caso 4 & PF \\
\hline 1 & 0,16 & 0,18 & 0,31 & 0,20 & 0,16 \\
\hline 2 & 0,42 & 0,48 & 0,73 & 0,55 & 0,45 \\
\hline 3 & 0,67 & 0,77 & 1,09 & 0,89 & 0,73 \\
\hline 4 & 0,88 & 1,03 & 1,40 & 1,18 & 0,97 \\
\hline 5 & 1,06 & 1,23 & 1,64 & 1,40 & 1,17 \\
\hline 6 & 1,19 & 1,39 & 1,81 & 1,58 & 1,33 \\
\hline 7 & 1,28 & 1,49 & 1,92 & 1,70 & 1,44 \\
\hline 8 & 1,34 & 1,56 & 1,98 & 1,78 & 1,53 \\
\hline
\end{tabular}

Observa-se uma melhor concordância entre os resultados obtidos via método P- $\Delta$ com os valores de EI recomendados por MACGREGOR \& HAGE (Caso 2). Já os valores obtidos com os valores de EI recomendados pelo ACI$318 / 89$ (Caso 3 ) superestimam os deslocamentos em $21 \%$, enquanto os valores obtidos no Caso 1 (FRANCO) subestimam os deslocamentos em $20 \%$.

TABELA 4.16 - Deslocamentos laterais do pórtico para o carregamento 2, via método $\mathrm{P}-\Delta$

\begin{tabular}{|c|c|c|c|c|c|}
\hline Andar & Caso 1 & Caso 2 & Caso 3 & Caso 4 & PF \\
\hline 1 & 0,32 & 0,35 & 0,62 & 0,40 & 0,33 \\
\hline 2 & 0,84 & 0,96 & 1,44 & 1,10 & 0,88 \\
\hline 3 & 1,35 & 1,55 & 2,18 & 1,78 & 1,40 \\
\hline 4 & 1,79 & 2,07 & 2,79 & 2,37 & 1,84 \\
\hline 5 & 2,14 & 2,49 & 3,27 & 2,85 & 2,19 \\
\hline 6 & 2,42 & 2,82 & 3,64 & 3,21 & 2,46 \\
\hline 7 & 2,60 & 3,04 & 3,87 & 3,46 & 2,65 \\
\hline $\mathbf{8}$ & 2,73 & 3,19 & 3,99 & 3,63 & 2,78 \\
\hline
\end{tabular}


Para o carregamento 2, observa-se uma boa concordância entre os deslocamentos obtidos pelo método P- $\Delta$ no Caso 1 (FRANCO) e aqueles fornecidos pelo programa PF. Nos demais casos, a diferença entre os valores obtidos via PF e aqueles através do método P- $\Delta$ varia de $15 \%$ (Caso 2) a $43 \%$ (Caso 3).

A comparação entre os resultados dos momentos fletores obtidos via método $\mathrm{P}-\Delta$ e aqueles fornecidos pelo programa $\mathrm{PF}$, estão mostrados nas tabelas 4.17 e 4.18 .

TABELA 4.17 - Momentos fletores no pórtico para o carregamento 1, via método $\mathrm{P}-\Delta$

\begin{tabular}{|c|c|c|c|c|c|}
\hline Seção & Caso 1 & Caso 2 & Caso 3 & Caso 4 & PF \\
\hline 9 & -146.13 & -145.54 & -148.61 & -145.62 & -138.52 \\
\hline 17 & -144.69 & -144.48 & -141.54 & -144.52 & -138.97 \\
\hline 1 & -45.70 & -48.99 & -29.83 & -48.89 & -50.69 \\
\hline 2 & -71.82 & -75.34 & -60.22 & -75.61 & -69.26 \\
\hline 3 & -32.83 & -35.25 & -28.70 & -35.52 & -32.97 \\
\hline 4 & -70.06 & -73.50 & -58.79 & -73.58 & -74.69 \\
\hline
\end{tabular}

Os resultados dos momentos fletores para o carregamento 1 obtidos pelo método $P-\Delta$ nos casos 1, 2 e 4 mostram uma boa correlação com aqueles obtidos pela análise não-linear via programa $\mathrm{PF}$. No Caso 3, os momentos nas colunas ficam subestimados em até $42 \%$ em relação aos obtidos na análise não-linear. Os esforços nas vigas (seções 9 e 17) para os 4 casos foram praticamente os mesmos, mostrando que a influência da fissuração foi maior nas colunas que nas vigas. 
TABELA 4.18 - Momentos fletores no pórtico para o carregamento 2, via método P- $\Delta$

\begin{tabular}{|c|c|c|c|c|c|}
\hline Seção & Caso 1 & Caso 2 & Caso 3 & Caso 4 & PF \\
\hline 9 & -144.87 & -142.99 & -152.85 & -143.05 & -127.96 \\
\hline 17 & -147.37 & -145.08 & -154.71 & -144.99 & -130.48 \\
\hline 1 & -29.68 & -35.57 & -5.26 & -35.45 & -26.57 \\
\hline 2 & -119.95 & -126.37 & -100.73 & -126.90 & -113.38 \\
\hline 3 & -88.40 & -93.89 & -75.24 & -94.40 & -86.94 \\
\hline 4 & -108.78 & -115.22 & -89.82 & -115.47 & -104.60 \\
\hline
\end{tabular}

Para o carregamento 2, os momentos fletores obtidos pelo método P- $\Delta$ para o Caso 1 (FRANCO) apresentam uma razoável concordância com aqueles obtidos pela análise não-linear (erro de 13\%). Nos demais casos, a diferença entre os valores obtidos via análise não-linear e aqueles fornecidos pelo método P- $\Delta$ varia de $34 \%$ (Caso 2) até $400 \%$ (Caso 3 ).

As Tab. 4.19 e 4.20 comparam os valores dos momentos fletores obtidos através da análise não-linear via programa $\mathrm{PF}$, com aqueles obtidos pelo método do amplificador de momentos de $1^{\underline{a}}$ ordem, conforme recomendado pela proposta de revisão do ACI-318/89, citada por MACGREGOR (1993).

TABELA 4.19 - Momentos fletores no pórtico para o carregamento 1, via método do amplificador de momentos

\begin{tabular}{|c|c|c|c|c|c|}
\hline Seção & Caso 1 & Caso 2 & Caso 3 & Caso 4 & PF \\
\hline 1 & $-45,33$ & $-48,49$ & $-29,53$ & $-48,32$ & $-50,69$ \\
\hline 2 & $-71,62$ & $-75,40$ & $-60,39$ & $-75,32$ & $-69,26$ \\
\hline 3 & $-32,62$ & $-35,27$ & $-28,87$ & $-35,22$ & $-32,97$ \\
\hline 4 & $-69,60$ & $-73,55$ & $-58,97$ & $-73,36$ & $-74,69$ \\
\hline
\end{tabular}


TABELA 4.20 - Momentos fletores no pórtico para o carregamento 2, via método do amplificador de momentos

\begin{tabular}{|c|c|c|c|c|c|}
\hline Seção & Caso 1 & Caso 2 & Caso 3 & Caso 4 & PF \\
\hline 1 & $-29,09$ & $-34,69$ & $-5,03$ & $-34,53$ & $-26,57$ \\
\hline 2 & $-119,13$ & $-126,06$ & $-100,27$ & $-125,94$ & $-113,38$ \\
\hline 3 & $-87,55$ & $-93,54$ & $-74,78$ & $-93,40$ & $-86,94$ \\
\hline 4 & $-108,11$ & $-114,97$ & $-89,54$ & $-114,70$ & $-104,60$ \\
\hline
\end{tabular}

Para o carregamento 1 observa-se uma boa concordância entre os valores obtidos pelo método do amplificador de momentos, exceto para o Caso 3 , onde a diferença varia de $15 \%$ a $72 \%$. Os resultados obtidos para o carregamento 2 têm boa concordância no Caso 1, nos demais casos o erro varia de $30 \%$ no Caso 4 até $\mathbf{4 2 8 \%}$ no Caso 3 . Pode-se portanto concluir que neste exemplo os resultados obtidos pelo método do amplificador de momentos não são totalmente confiáveis.

O parâmetro de estabilidade "Q" para o $1^{\circ}$ pavimento varia de 0,04 a 0,08 para o carregamento 1 e de 0,03 a 0,06 para o carregamento 2 .

Finalmente, com a finalidade de averiguar a precisão do método P- $\Delta \mathrm{e}$ do método do amplificador de momentos para níveis de carga próximos do colapso, a estrutura foi reavaliada considerando-se o carregamento original multiplicado por fator 2 . Considerou-se na análise em $1^{\underline{a}}$ ordem as inércias fissuradas do Caso 2 (MACGREGOR).

A Tab. 4.21 mostra uma comparação entre os deslocamentos fornecidos pelo programa PF e aqueles obtidos pelo método $\mathrm{P}-\Delta$. 
TABELA 4.21 - Deslocamentos laterais do pórtico com carregamento multiplicado por 2 , via método $P-\Delta$

\begin{tabular}{|c|c|c|c|c|c|c|}
\hline \multirow{2}{*}{ Pavimento } & \multicolumn{3}{|c|}{ Carregamento 1 } & \multicolumn{3}{c|}{ Carregamento 2 } \\
\cline { 2 - 7 } & $1^{\mathrm{a}}$ Ordem & P- $\Delta$ & PF & $1^{\mathrm{a}}$ Ordem & P- $\Delta$ & PF \\
\hline 8 & 2.94 & 3.32 & 4.97 & 5.89 & 6.52 & 7.95 \\
\hline 7 & 2.82 & 3.18 & 4.64 & 5.64 & 6.25 & 7.59 \\
\hline 6 & 2.62 & 2.96 & 4.27 & 5.23 & 5.82 & 7.10 \\
\hline 5 & 2.32 & 2.64 & 3.78 & 4.65 & 5.18 & 6.37 \\
\hline 4 & 1.93 & 2.20 & 3.14 & 3.87 & 4.32 & 5.37 \\
\hline 3 & 1.45 & 1.66 & 2.35 & 2.91 & 3.25 & 4.08 \\
\hline 2 & 0.90 & 1.02 & 1.40 & 1.80 & 2.00 & 2.51 \\
\hline 1 & 0.33 & 0.38 & 0.43 & 0.68 & 0.74 & 0.88 \\
\hline
\end{tabular}

Neste caso, os resultados do método P- $\Delta$ subestimam os deslocamentos em até $50 \%$ para o carregamento $1 \mathrm{e}$ em até $22 \%$ para o carregamento 2 .

A correlação entre os momentos fletores nas colunas e viga do $1^{\circ}$ pavimento obtidos via programa $P F$ e aqueles fornecidos pelo método $P-\Delta$ está mostrada na Tab. 4.22.

TABELA 4.22 - Momentos fletores no pórtico com carregamento multiplicado por 2 , via método $\mathrm{P}-\Delta$

\begin{tabular}{|c|c|c|c|c|c|c|}
\hline \multirow{2}{*}{ Seção } & \multicolumn{3}{|c|}{ Carregamento 1 } & \multicolumn{3}{c|}{ Carregamento 2} \\
\cline { 2 - 7 } & $1^{\mathrm{a}}$ Ordem & $\mathrm{P}-\Delta$ & $\mathrm{PF}$ & $1^{\mathrm{a}}$ Ordem & $\mathrm{P}-\Delta$ & $\mathrm{PF}$ \\
\hline 1 & -97.37 & -98.95 & -121.33 & -70.04 & -72.54 & -73.55 \\
\hline 2 & -145.46 & -157.01 & -160.00 & -243.24 & -262.68 & -253.35 \\
\hline 3 & -65.25 & -76.88 & -75.24 & -178.66 & -198.10 & -189.76 \\
\hline 4 & -142.63 & -152.30 & -179.55 & -222.84 & -239.26 & -241.91 \\
\hline 9 & -288.82 & -293.991 & -279.36 & -282.00 & -290.64 & -262.74 \\
\hline 17 & -286.54 & -291.99 & -282.65 & -285.90 & -295.15 & -268.47 \\
\hline
\end{tabular}


Observa-se, neste caso, uma variação na precisão dos resultados obtidos pelo método $\mathrm{P}-\Delta$, que varia de $2 \%$ na seção 3 e carregamento 1 , até $20 \%$ na seção 1 e carregamento 1 . Na seção 4 (coluna) o momento é subestimado em 15\% (carregamento 1). As seções 9 e 17 (viga) têm os momentos superestimados em até $10 \%$. Apesar das variações de precisão observadas pode-se concluir que os resultados obtidos pelo método $\mathrm{P}-\Delta$ mesmo nas proximidades da carga de colapso podem ser considerados aceitáveis a nível de projeto.

Na Tab. 4.23 mostra-se a correlação entre os momentos nas colunas do $1^{\circ}$ pavimento obtidos pelo método do amplificador de momentos e aqueles fornecidos pelo programa PF.

TABELA 4.23 - Momentos fletores no pórtico com carregamento multiplicado por 2, via método do amplificador de momentos

\begin{tabular}{|c|c|c|c|c|}
\hline \multirow{2}{*}{ Seção } & \multicolumn{2}{|c|}{ Carregamento 1 } & \multicolumn{2}{c|}{ Carregamento 2 } \\
\cline { 2 - 5 } & Amplificador & PF & Amplificador & PF \\
\hline 1 & -96.70 & -121.33 & -68.85 & -73.55 \\
\hline 2 & -154.21 & -160.00 & -259.30 & -253.35 \\
\hline 3 & -73.98 & -75.24 & -194.24 & -189.76 \\
\hline 4 & -149.99 & -179.55 & -235.98 & -241.91 \\
\hline
\end{tabular}

Para o carregamento 1 , o método do amplificador de momentos subestima os momentos em até $25 \%$ (Seção 1) e, para o carregamento 2 , o erro não atinge $7 \%$, mostrando-se assim que a precisão deste método para carregamento próximo do colapso é, no mínimo, duvidosa.

Para este nível de carregamento, o parâmetro de estabilidade "Q" para o $1^{0}$ pavimento é igual a 0,083 para o carregamento 1 e 0,064 para o carregamento 2 . 
Em todas as análises via $\mathrm{PF}$, utilizando-se um microcomputador Pentium $90 \mathrm{MHz}$, o carregamento foi aplicado em incrementos de carga de 5\% do carregamento total. $O$ tempo gasto em cada iteração foi de 20 segundos, a convergência foi atingida com, no máximo, 4 iterações em cada incremento de carga; assim, o tempo de processamento foi de aproximadamente 25 minutos. Nas proximidades da carga de colapso, a convergência se deu com até 12 iterações. 


\section{CONCLUSÕES E RECOMENDAÇÕES}

Este trabalho apresenta uma contribuição ao estudo da não-linearidade física e geométrica em pórticos planos de concreto armado utilizando-se uma formulação teórica consistente.

Inicialmente apresentou-se uma revisão dos métodos aproximados para consideração dos efeitos não-lineares nestas estruturas conforme recomendação das principais normas internacionais.

Uma formulação consistente foi desenvolvida com base na teoria de grandes deslocamentos e grandes deformações, aplicada a elementos de pórtico plano. O desenvolvimento teórico foi feito dentro da formulação Lagrangeana parcialmente atualizada. A matriz de rigidez do elemento foi obtida utilizandose um sistema de coordenadas corrotacional, solidário ao elemento, considerando-se apenas os graus de liberdade naturais. A partir da teoria estrutural de Euler-Bernoulli-Navier define-se o campo de deformações do elemento. Utilizando-se o Princípio dos Trabalhos Virtuais formulou-se o equilibrio elemental. Para interpolação dos deslocamentos foram introduzidas funções aproximadoras lineares para os deslocamentos axiais e cúbicas para os deslocamentos transversais.

Algumas aproximações de natureza geométrica e algumas simplificações no comportamento do elemento foram feitas para facilitar a implementação, sem que a formulação perdesse a generalidade. Um elemento prismático em regime elasto-plástico foi então obtido. Através da matriz de incidência cinemática foi feita a compatibilidade entre os deslocamentos nodais da estrutura e os deslocamentos nodais do elemento. Usando-se o Princípio dos Trabalhos Virtuais chegou-se à equação de equilíbrio estrutural, e a partir desta à equação de equilíbrio incremental. 
A forma incremental do método iterativo de Newton-Raphson foi implementada permitindo-se assim acompanhar o comportamento não-linear da estrutura até o colapso.

Para verificação numérica da formulação proposta foi implementado computacionalmente um programa de análise. Exemplos numéricos ilustraram a aplicabilidade e a potencialidade da formulação, comparando-se os resultados numéricos de estruturas submetidas a grandes deslocamentos, com aqueles fornecidos por soluções analíticas disponíveis na literatura.

A formulação foi então estendida à análise de pórticos planos de concreto armado, utilizando-se o processo das fatias para discretização da seção transversal. A equação constitutiva utilizada para descrever o comportamento do concreto em compressão uniaxial, foi aquela proposta pela NBR-6118, e para o concreto não fissurado em tração, a equação recomendada pelo CEB MC 90, desprezando-se os efeitos do enrijecimento devido ao concreto intacto entre as fissuras ("tension stiffening"). Admitiu-se ainda a perfeita aderência entre as barras de aço e o concreto.

Finalmente foram apresentados alguns exemplos de pórtico planos de concreto armado com o propósito de mostrar a boa correlação entre os dados obtidos através da formulação aqui desenvolvida, e resultados experimentais e numéricos fornecidos por outros pesquisadores. Através destes exemplos mostrou-se ainda o desempenho e a precisão dos principais métodos aproximados de avaliação dos efeitos não-lineares em pórticos planos de concreto armado de acordo com as recomendações das normas.

O objetivo predominante em todas as fases do trabalho foi apresentar uma contribuição ao desenvolvimento de modelos de análise não-linear de estruturas de concreto armado, que nos parece ser um imperativo da engenharia moderna. 
As contribuições principais deste trabalho pretende-se que sejam:

- O desenvolvimento de uma formulação teoricamente consistente aplicada a estruturas planas de concreto armado, levando-se em consideração o comportamento não-linear físico e geométrico, assim como a deformabilidade dos apoios, incluindo a previsão da carga última;

- A elaboração de um programa ("PF") para análise não-linear incremental, e iterativa de pórticos planos de concreto armado utilizando-se microcomputadores, objetivando-se a utilização em escritórios de projeto, e difundindo-se assim o uso da análise não-linear;

- A delimitação do campo de aplicação das ferramentas aproximadas usuais existentes para análise não-linear de pórticos de concreto armado, principalmente o conhecido "método P- $\Delta$ ".

A formulação desenvolvida neste trabalho $\mathrm{e}$ implementada computacionalmente, mostrou-se bastante eficiente e poderosa como foi demonstrado nos exemplos apresentados.

Nos exemplos apresentados no Capítulo III fica destacada a eficiência do programa e sua capacidade de seguir corretamente o comportamento nãolinear das estruturas analisadas em regime de grandes deslocamentos. Os resultados destes exemplos mostram que para análise não-linear geométrica, (ANLG) a discretização das barras com um elemento é suficiente quando a estrutura está submetida a deslocamentos de até $12 \%$ do comprimento da barra. Ficou evidente ainda que, à medida que aumenta a grandeza dos deslocamentos uma discretização com mais elementos se faz necessária, porém não mais que quatro elementos por barra. Considerando-se que as estruturas reais da prática dificilmente atingem deslocamentos superiores a $12 \%$ do vão, pode-se concluir que quando a estrutura só apresenta não-linearidade geométrica, a discretização com um elemento por barra é suficiente para se obter bons resultados. 
Nos exemplos apresentados no Capítulo IV ficou evidenciada a boa correlação entre os resultados obtidos através do programa desenvolvido, com os fornecidos em análises experimentais e numéricas feitas por outros pesquisadores. Estes exemplos mostraram ainda a capacidade do programa de seguir corretamente o comportamento não-linear físico e geométrico dos pórtico planos de concreto armado nas fases elástica e fissurada, até a ruptura.

Com base nos exemplos 1 e 2 do Capítulo IV, pode-se concluir que o modelo constitutivo do concreto, que considera sua resistência à tração superestima a carga de ruptura do pórtico enquanto o modelo que não considera a resistência à tração subestima esta carga. O segundo modelo apresentou resultados mais próximos dos experimentais. Assim é recomendável que, em nível de projeto, não se considere a resistência do concreto à tração.

O exemplo 3 do Capítulo IV mostrou a importância da discretização longitudinal das barras em vários elementos, principalmente quando há flexão anti-simétrica. Ficou evidenciado que 10 elementos por barra é suficiente para uma solução com precisão adequada. Mostrou-se ainda que a discretização da seção transversal em 10 fatias é suficiente, uma vez que uma grande discretização da seção implica maior tempo computacional sem ganhos significativos de precisão.

Os exemplos 4 e 5 do capítulo mostraram que o programa desenvolvido pode ser utilizado para análise não-linear de pórticos de edifícios altos de concreto armado. Obteve-se uma boa correlação entre os resultados fornecidos pelo programa e aqueles obtidos por CILONI (1993) e CAUVIN (1978), sendo que os deslocamentos obtidos nestas referências foram um pouco menores, o que se justifica pois o modelo constitutivo para o concreto utilizado por eles considera a contribuição do concreto intacto entre fissuras ("tension stiffening”). Para os pórticos estudados, a análise não-linear pelo método P- $\Delta$ ofereceu bons resultados se comparados aos obtidos pelo programa "PF", 
principalmente para cargas de serviço e quando se considerou valores adequados para a rigidez efetiva EI. Das várias recomendações para a consideração da rigidez efetiva da seção fissurada, a que apresentou melhor resultado foi a de MACGREGOR \& HAGE (1977), ou seja, EI = 0,4 $E_{c} I_{g}$ para vigas e $\mathrm{EI}=0,8 \mathrm{E}_{\mathrm{c}} \mathrm{I}_{\mathrm{g}}$ para colunas. Observa-se ainda que os valores de $\mathrm{EI}$ recomendados pelo item 10.10.1 do ACl-318/89 (Eq. 2.28) para colunas são muito baixos levando-se a uma superestimação dos deslocamentos e a uma inadequada distribuição de momentos. Os valores recomendados por FRANCO \& VASCONCELOS (1991) e na proposta de revisão do ACI-318/89 levaram a resultados compatíveis. Para um nível de carregamento próximo ao colapso, o método P- $\Delta$ apresentou resultados que subestimaram os deslocamentos laterais ( $50 \%$ no caso do exemplo 5 ), mas em termos de momentos fletores, mesmo para este nível de cargas, os resultados foram satisfatórios. O método do amplificador de momentos apresentou resultados com uma razoável concordância para cargas de serviço, mas pareceu não ser totalmente confiável. Estes exemplos evidenciaram ainda a importância dos efeitos da fissuração na análise de pórticos de edifícios altos em concreto armado, e essa importância é maior para as colunas que para as vigas.

Para o pórtico do exemplo 4, o recalque vertical de $2 \mathrm{~cm}$ num apoio reduziu a carga última em $8 \%$, mas com grande aumento nos deslocamentos, enquanto para o pórtico do exemplo 5, mais rígido, este mesmo recalque reduziu a carga última à metade. Estes exemplos mostram a importância da deformabilidade das fundações no comportamento de estruturas de edifícios altos.

Embora, na implementação executada não tenha sido considerada como prioritária a otimização do ponto de vista computacional, mas a versatilidade para fins de análise de comportamento estrutural, os tempos de processamento obtidos indicam a viabilidade prática de análises não-lineares de pórticos de 
edifícios altos de concreto armado, possibilitando-se a elaboração de estruturas mais esbeltas, econômicas e com segurança adequada.

Em termos de sugestões de trabalhos futuros para continuidade dos estudos aqui realizados, citam-se:

- a extrapolação deste modelo para estruturas tridimensionais de edifícios altos através da associação de pórticos planos;

- consideração de modelos constitutivos mais realísticos para o concreto baseado na teoria do dano;

- inclusão dos efeitos reológicos (deformação lenta e retração);

- inclusão das deformações por cisalhamento nos elementos;

- incorporar ao trabalho desenvolvido, a possibilidade de análise dinâmica, onde os efeitos não-lineares seriam muito importantes;

- estender o modelo a estruturas em grelhas com inclusão da torção, possibilitando-se a análise de pavimentos de edifícios. 


\section{REFERÊNCIAS BIBLIOGRÁFICAS}

1 AAS-JAKOBSEN, K. ; GRENACHER. Analysis of slender reinforced concrete frames. International Association for Bridge and Structural Engineering Publications, v. 34, p. 1-17, 1974.

2 AL-SHAIKH, A. H. ; AL-ZAID, R. Z. Effect of reinforcement ratio on the effective moment of inertia of reinforced concrete beams. ACI Structural Journal, p. 144-149, March-April, 1993.

3 ALVAREZ, R. J. ; BIRNSTIEL, C. Inelastic analysis of multistory multibay frames. Journal of the Structural Division (ASCE), v. 95, n.St 11, p. 2477-2503, November, 1969.

4 ALWIS, W. A. M. Trilinear moment-curvature relationship for reinforced concrete beams. ACI Structural Journal, p. 276-283, MayJune, 1990.

5 AMERICAN CONCRETE INSTITUTE (ACI). Building code requirements for reinforced concrete. 318R-89-Revised 1992.

6 AMERICAN INSTITUTE OF STEEL CONSTRUCTION (AISC). Load and resistance factor design - Specification for structural steel building. 1.ed., 1986.

7 ANTUNES, H. M. C. C. Carregamento crítico de instabilidade geral para estruturas tridimensionais de edificios altos. São Carlos, 1978. 160p. Tese (Doutorado) -Escola de Engenharia de São Carlos/USP.

8 ARAÚJO, M. J. Optimization of newton-raphson methods in nonlienar analysis. Computers \& Structures, v. 33, n. 3, p. 735-741, 1989.

9 ARGYRIS, J. H. Continua and discontinua. Proc. Conf. on Matrix Methods in Structural Mechanics Wright-Patterson A.F.B. , Ohio , p.11185 , October, 1965.

10 ARGYRIS, J. H. ; DUNNE, P. C. ; MALEJANNAKIS, G. A. ; SCHARPF, D. W. Finite element methods - the natural aproach. Computer Methods in Applied Mechanics and Engineering, v. 17/18, p. 1-106, 1979a. 
11 ARGYRIS, J. H. ; HILPERT, O. ; MALEJANNAKIS, G. A. ; SCHARPF, D. W. On the geometrical stiffiness of a beam in space - A consistent V. W. Approach. Computer Methods in Applied Mechanics and Engineering, v. 20, p. 105-131, 1979 b.

12 ARGYRIS, J. H. ; KELSEY, S. Energy theorems and structural analysis, v. 26/27, Part I por J.H. Argyris e Part II por J.H. Argyris e S. Kelsey, October 1954 a May 1955.

13 ASSAN, A. E. Matriz de rigidez de elemento finito tridimensional de viga para análise não linear física e geométrica. In: CONGRESSO IBERO LATINO AMERICANO SOBRE MÉTODOS COMPUTACIONAIS PARA ENGENHARIA, 11., Rio de Janeiro, 1990. Anais. Rio de Janeiro, COPPE-UFRJ/AMC, 1990. v. 2, p.707-716.

14 ASSAN, A. E. Vigas de concreto armado com não linearidade física. In: CONGRESSO IBERO LATINO AMERICANO SOBRE MÉTODOS COMPUTACIONAIS PARA ENGENHARIA, 11., Rio de Janeiro, 1990. Anais. Rio de Janeiro, COPPE-UFRJ/AMC, 1990. v. 2, p.741-749.

15 ASSOCIAÇÃO BRASILEIRA DE NORMAS TÉCNICAS. Projeto $e$ execução de obras de concreto armado - NBR 6118. Rio de Janeiro, 1980.

16 BALAKRISNAN, S. ; ELWI, A. E. ; MURRAY, D. W. Effect of modeling on NLFE analysis of concrete structures. Journal of Structural Engineering, v. 114, n.7, p. 1467-1487, July, 1988.

17 BALAKRISNAN, S. ; MURRAY, D. W. Concrete constitutive model for NLFE analysis of structures. Journal of Structural Engineering, v.114, n.7, p. 1449-1466, July, 1988.

18 BATHE, K. J. Finite element procedures. ( Revision of : Finite element procedures in engineering analysis, 1982 ); New Jersey, Prentice Hall, Inc. , Englewood Cliffs, 1996.

19 BATHE, K. J. ; BOLOURCHI, S. Large displacement analysis of threedimensional beam structures. International Journal for Numerical Methods in Engineering, v. 14, p. 961-986, 1979.

20 BATHE, K. J. ; CIMENTO, A. P. Some pratical procedures for the solution of nonlinear finite element equations. Computer Methods in Applied Mechanics and Engineering, v. 22, n.1, p. 59-85, 1980. 
21 BATHE, K. J. ; DVORKIN, E. N. On the automatic solution of nonlinear finite element equations. Computer \& Structures, v. 17, n. 5/6, p.871-879, 1983.

22 BATHE, K. J. ; RAMM, E. ; WILSON, E. L. Finite element formulation for large deformation dynamic analysis. International Journal for Numerical Methods in Engineering, v. 9, p. 353-386, 1975.

23 BATHE, K. J. et al. Nonlinear analysis of concrete structures. Computer \& Structures, v. 32, n. 3/4, p. 563-590, 1989.

24 BECK, H. ; KONIG, G. Restraining forces in the analysis of tall buildings. In: SYMPOSIUM ON TALL BUILDINGS. Oxford, 1966. p.513-536.

25 BOYLE, C. ; JENNINGS, A. Accelarating the convergence of elasticplastic stress analysis. International Journal for Numerical Methods in Engineering, v. 7, p. 232-235, 1973.

26 BREBBIA, C. A. ; CONNOR, J. J. Fundamentals of finite element techniques. Butter- worths \& Co.(Publishers) Ltda, 1973.

27 CAUVIN, A. Non linear analysis of a multistorey sway frame according to CEB model code. CEB: Bulletin D'Information, n.134, p. 83-107, 1979.

28 CEDOLIN, L. ; NILSON, A. H. A convergence study of iterative methods applied to finite element analysis of reinforced concrete. International Journal for Numerical Methods in Engineering, v. 12, p.437-451, 1978.

29 CHEN, W. F. Plasticity in reinforced concrete. New York, McGrawHill, 1982.

30 CHEN, W. F. ; HAN, D.J. Plasticity for structural engineers. New York, Spring-Verlag, 1988.

31 CHEN, W. F. ; LUI ; E. M. Structural stability - Theory and implementation. New York, Elsevier, 1987.

32 CHEN, Z. Q. ; AGAR, T. J. A. Geometric nonlinear analysis of flexible spatial beam structures. Computeres \& Structures, v. 49, n. 6, p.10831094, 1993. 
33 CILONI, A. D. Sobre o comportamento em serviço de estruturas planas de concreto armado. São Carlos, 1993. Tese (Doutorado) - Escola de Engenharia de São Carlos, Universidade de São Paulo.

34 COMITÉ EURO-INTERNATIONAL DU BÉTON. CEB-FIP Manual of buckling and instability. Lancaster, The construction Press Ltd, 1978.

35 COMITÉ EURO-INTERNATIONAL DU BÉTON. CEB-FIP Model Code 1990. CEB: Bulletin D'Information, n. 203/205, July, 1991.

$36 \mathrm{CONCI}, \mathrm{A}$. Análise de estruturas reticuladas de aço com consideração de empenamento e não-linearidade geométrica e material. Rio de Janeiro, 1988. Tese (Doutorado) - Pontifícia Universidade Católica/RJ.

37 CONCI, A. ; GATTASS, M. Natural approach for thin-walled beamcolumns with elastic-plasticity. International Journal for Numerical Methods in Engineering, v. 29, p. 1653-1679, 1990.

38 CONCI, A. ; GATTASS, M. Natural approach geometric nonlinear analysis of thin-walled frames. International Journal for Numerical Methods in Engineering, v. 30, p. 207-231, 1990.

39 CORRADI, L. ; DONATO, O. ; MAIER, G. Inelastic analysis of reinforced concrete frames. Journal Structural Division, v. 100, n. 9, p. 1925-1943, 1974.

40 CORRÊA, M. R. S. Aperfeiçoamento de modelos usualmente empregados no projeto de sistemas estruturais de edificios. São Carlos, 1991. Tese (Doutorado) - Escola de Engenharia de São Carlos, Universidade de São Paulo.

41 CORREAA, M. R. S. ; RAMALHO, M. A. Modelos numéricos para análise estrutural de edifícios. In: SEMINÁRIO SOBRE NÃOLINEARIDADE FÍSICA E GEOMÉTRICA DAS ESTRUTURAS DE CONCRETO, IBRACON. São Paulo, 1995.

42 CRISFIELD, M. A. A fast incremental/iterative solution procedure that handles "snap-through". Computers \& Structures, v. 13, p. 55-62, 1981.

43 CRISFIELD, M. A. Solution procedures for non-linear structural analysis. In: HINTON, E. ; OWEN, D. R. J. ; TAYLOR, C. ed. Recent advances in nonlinear computational mechanics. Swansea, UK: Pineridge Press, 1982. p. 1-39. 
44 DESAI, C. S. ; ABEL, J. F. Techniques for nonlinear analysis. Introduction to the finite element method: a numerical method for engineering analysis. New York, Van Nostrand Reinhold, 1972.

45 DIAZ, M. A. Evaluation of approximate slenderness procedure for nonlinear analysis of concrete and steel frames. Austin, 1984. PhD (Dissertation) - University of Texas.

46 DIAZ, M. A. ; ROESSET, J. M. Evaluation of aproximate slenderness procedures for nonlinear analysis of concrete frames. ACI Structural Journal, p. 139-148, March-April, 1987.

47 EL-METWALLY, S. E. ; CHEN, W. F. Nonlinear behavior of R/C frames. Computers \& Structures, v. 32, n.6, p. 1203-1209, 1989.

48 ERNST, C. ; SMITH, M. ; RIVELAND, R. ; PIERCE, N. Basic reinforcement concrete frames performance under vertical and lateral loads. ACI Journal, v. 70, n. 4, p. 261-269, April, 1973.

49 FONTE, A. C. C. Análise não-linear geométrica de edificios altos. Rio de Janeiro, 1992. Tese (Doutorado) - COPPE, Universidade Federal do Rio de Janeiro.

50 FRANCO, M. Problemas de estabilidade nos edifícios de concreto armado. In: COLÓQUIO SOBRE ESTABILIDADE GLOBAL DAS ESTRUTURAS DE CONCRETO ARMADO, IBRACON, São Paulo, 1985 .

51 GALlAGHER, R. H. ; PADLOG, J. ; BIJLAARD, P. P. Stress analysis of heated complex shapes. American Rocket Society Journal, v. 32, p.700-707, May, 1962.

52 GATASS, M. ; ABEL, J. F. Equilibrium considerations of the updated Lagrangian formulation of beam-columns with natural concepts. International Journal for Numerical Methods in Engineering, v. 24, p.2119-2141, 1987.

53 GREUNEN, J. V. ; SCORDELIS, A. C. Nonlinear analysis of prestressed concrete slabs. Journal of Structural Engineering, v.109, n.7, p. 1742-1760, July, 1983.

54 HINTON, E. ; OWEN, D. R. J. An introduction to finite element computations. Swansea, U. K., Pineridge Press, 1979. 
55 HINTON, E. ; OWEN, D. R. J. ; TAYLOR, C. Recent advances in nonlinear computational mechanics. Swansea, U. K., Pineridge Press, 1982.

56 HOGNESTAD, E. A study of combined bending and axial load in reinforced concrete members. Bulletin n. 399. Engineering Experiment Station. University of Illinois, Urbana, 1951.

57 HORNE, M. R. An approximate method for calculing the elastic critical loads of multistory plane frames. The structural engineer, v. 53, n. $6, \mathrm{p}$. 242-248, June, 1975.

58 HORNE, M. R. Plastic theory of structures. 2 ed., Pergamon Press, 1979.

59 HSIAO, K. M. ; HORNG, H. J. ; CHEN Y. H. A corotational procedure that handles large rotation of spatial beam structures. Computers \& Structures, v. 27, n. 6, p. 769-781, 1987.

60 IZZUDDIN, B. A. ; KARAYANNIS, C. G. ; ELNASHAI, A. S. Advanced nonlinear formulation for reinforced concrete beam-columns. Journal of Structural Engineering, v. 120, n. 10, p. 2913-2934, October, 1994.

61 JENNINGS, A. Frame analysis including change of geometry. Journal of the Structural Division (ASCE), v. 94, n. St 3, p. 627-694, March, 1968.

62 JORDAN S-M., R. ; KREGER, M. E. Modelacion de uniones vigacolumna de porticos de hormigon armado. In: JORNADAS SULAMERICANAS DE ENGENHARIA ESTRUTURAL, 25., Porto Alegre, 1991. Anais. Porto Alegre, CPGEC-UFRGS, 1991. v. 1, p. 247-258.

$63 \mathrm{KAM}, \mathrm{T}$. Y. Large deflection analysis of inelastic plane frames. Journal of Structural Engineering, v. 114, n. 1, p. 114-197, January, 1988.

64 KANG, Y. J. ; SCORDELIS, A. C. Nonlinear analysis of prestressed concrete frames. Journal of the Structural Division (ASCE), v. 106, p.445-462, February, 1980.

65 KARAYANNIS, C. G. ; IZZUDDIN, B. A. ; ELNASHAI, A. S. Aplication of adaptive analysis to reinforced concrete frames. Journal of Structural Engineering, v. 120, n. 10, p. 2935-2957, October, 1994. 
66 KASSIMALI, A. Large deformations analysis of elastic-plastic frames. Journal of the Structural Engineering (ASCE), v. 109 ,n. 8, p. 1869-1887, August, 1983.

67 KAYAL, S. Finite element analysis of RC frames. Journal of Structural Engineering, v. 110, n. 12, p. 2891-2908, December, 1984.

68 KRISHNAMOORTHY, C. S. ; MOSI, D. R. C. A computer program for inelastic analysis of reinforced concrete framed structures. Computers \& Structures, v. 12, p. 677-687, 1980.

69 KUO, S. R. ; YANG, Y. - B. ; CHOU, J. - H. Nonlinear analysis of space frames with finite rotation. Journal of Structural Engineering (ASCE), v. 119, n. 1, p. 1-15, January, 1993.

70 LAVALL, A. C. C. Análise elástica em segunda ordem de pórticos planos metálicos. São Carlos, 1989. 198p. Dissertação (Mestrado) Escola de Engenharia de São Carlos, Universidade de São Paulo.

71 LO, S. H. Geometrically nonlinear formulation of 3-D finite strain beam element with large rotations. Computers \& Structures, v. 44, n. $1 / 2$, p. 147-157, 1992.

72 MACGREGOR, J. G. Design of slender concrete columns revisited. $A C I$ Structural Journal, p. 302-308, May-June, 1993.

73 MACGREGOR, J. G. ; BREEN, J. E. ; PFRANG, E. O. Design of slender concrete columns. ACI Journal, v. 67, n. 1, p. 6-28, 1970.

74 MACGREGOR, J. G. ; HAGE, S. Stability analysis and design of concrete frames. Journal of Structural Division (ASCE), v. 103, n. 10, p. 1953-1970, October, 1977.

75 MALLETT, R. H. ; MARCAL, P. V. Finite element analysis of nonlinear structures. Journal of Structural Division (ASCE), v. 94,n. St 9 , p.2081-2105, September, 1968.

76 MANTILLA, E. P. Um método iterativo para análise da não-linearidade geométrica em estruturas reticuladas. Rio de Janeiro, 1974. Dissertação (Mestrado) - COPPE, Universidade Federal do Rio de Janeiro. 
77 MARÍ, A. R. ; CHAN, E. C. ; SCORDELIS, A. C. Nonlinear material, geometric and time dependent analysis of 3-D reinforced and prestressed concrete frames. In: TAYLOR, C. ; HINTON, E. ; OWEN, D. R. J. ed. Numerical methods for nonlinear problems. Swansea, UK, Pineridge Press, 1984. v. 2, p. 339-361.

78 MARTINS, P. C. R. A não-linearidade nas estruturas de concreto armado e protendido numa análise até a ruptura. In: SEMINÁRIO SOBRE NÃOLINEARIDADE FÍSICA E GEOMÉTRICA DAS ESTRUTURAS DE CONCRETO, IBRACON. São Paulo, 1995.

79 MARTINS, P. C. R. Análise não-linear de estruturas reticulares espaciais de concreto armado. Rio de Janeiro, 1979. Dissertação (Mestrado) COPPE, Universidade Federal do Rio de Janeiro.

80 MATTHIES, H. ; STRANG, G. The solution of nonlinear finite element equations. International Journal for Numerical Methods in Engineering, v. 14, n. 11, p. 1613-1626, 1979.

81 MATTIASSON, K. Numerical results from large deflection beams and frame problems analysed by means of elliptic integrals. International Journal for Numerical Methods in Engineering, v. 17, n. 1, p. 145-153, 1981.

82 MAZZILLI, C. E. N. Comportamento não-linear de pórticos planos em regime elástico linear. In: CONGRESSO LATINO-AMERICANO DE MÉTODOS COMPUTACIONAIS PARA A ENGENHARIA, 7., São Carlos, 1986.

83 MAZZILLI, C. E. N. Considerações sobre não-linearidade geométrica em estruturas reticuladas planas. São Paulo, EPUSP, 1987. (Boletim Técnico do Departamento de Engenharia de Estruturas e Fundações, 8701)

84 MEEK, J. L. ; LOGANATHAN, S. Geometric and material non-linear behavior of beam-columns. Computer and structures, v. 34, n.1, p.87$100,1990$.

85 MEEK, J. L. ; LOGANATHAN, S. Large displacement analysis of space-frame structures. Computer methods in applied mechanics and engineering, v. 72, p. 57-75, 1989. 
86 MENDIS, P. A. ; DARVALL, P. L. Stability analysis of softening frames. Journal of Structural Engineering (ASCE), v. 114, n. 5, p. 10571072, May, 1988.

87 MENEZES, F. A. ; ARCARO, V. Pórticos planos: grandes deslocamentos e deformações. In: JORNADAS SUL-AMERICANAS DE ENGENHARIA ESTRUTURAL, 25., Porto Alegre. Anais. Porto Alegre, CPGEC-UFRGS, 1991. v. 1, p. 69-80.

88 MORI, D. D. Os núcleos estruturais e a não-linearidade geométrica na análise de estruturas tridimensionais de edificios altos. São Carlos, 1992. 195p. Tese (Doutorado) - Escola de Engenharia de São Carlos, Universidade de São Paulo.

89 MULLER, H. S. ; HILSDORF, H. K. Constitutive relations for concrete. In: COLLOQUIUM ON THE CEB-FIP MODEL CODE 90, COPPE/CEB, Rio de Janeiro, Agosto, 1991.

90 NAGTEGAAL, J. C. Some recent developments in combined geometric and nonlinear finite element analysis. IN: HINTON, E. ; OWEN, D. R. J. ; TAYLOR, C. ed. Recent advances in nonlinear computational mechanics. Swansea, UK, Pineridge Press, 1982. p. 87-117.

91 NGO, D. ; SCORDELIS, A. C. Finite element analysis of reinforced concrete beams. ACI Journal, v. 64, n.3, p. 152-163, 1967.

92 ORAN, C. Tangent stiffiness in space frames. Journal of the structural divisiion (ASCE), v. 99, n. St 6, p. 987-1001, June, 1973.

93 ORAN, C. Tangent stiffness in plane frames. Journal of the structural division (ASCE), v. 99, n. St 6, p. 973-985, June, 1973.

94 ORAN, C. ; KASSIMALI, A. Large deformation of framed structures under static and dynamic loads. Computer \& Structures, v. 6, p.539-547, 1976.

95 OWEN, D. R. J. ; GOMES, C. M. B. Some recent developments in solution techniques for nonlinear finite element problems. Swansea, Department of Civil Engineering, University College of Swansea, 1984.

96 OWEN, D. R. J. ; GONÇALVES FILHO, O. J. A. Substructuring techniques in material nonlinear analysis. Computer \& Structures, v.15, n. 3, p. 205-213, 1982. 
97 OWEN, D. R. J. ; HINTON, E. Finite elements in plasticity: Theory and practice. Swansea U.K., Pineridge Press Ltd, 1980.

PAI, P. F. ; NAYFEH, A. H. A new method for the modeling of 98 geometric nonlinearities in structures. Computer \& structures, v. 53, n. 4, p.877-895, 1994.

99 PIMENTA, P. M. Análise não-linear de pórticos planos. São Paulo, EPUSP, 1986a. (Boletim Técnico do Departamento de Engenharia de Estruturas e Fundações, 8611).

100 PIMENTA, P. M. Análise não-linear de treliças espaciais. São Paulo, EPUSP, 1986b. (Boletim Técnico do Departamento de Engenharia de Estruturas e Fundações, 8604).

101 PIMENTA, P. M. Aspectos da análise não-linear de estruturas reticuladas. In: CONGRESSO LATINO-AMERICANO SOBRE MÉTODOS COMPUTACIONAIS PARA A ENGENHARIA, 7.,São Carlos, 1986c. p. 449-464.

102 PIMENTA, P. M. Análise não-linear de arcos. São Paulo, EPUSP, 1988. (Boletim Técnico do Departamento de Engenharia de Estruturas e Fundações, 8816).

103 PIMENTA, P. M. Derivation of tangent stiffness matrices of simple finite elements, I. Straight bar elements. São Paulo, EPUSP, 1989a. (Boletim Técnico do Departamento de Engenharia de Estruturas e Fundações, 8912).

104 PIMENTA, P. M. Nonlinear analysis of plane frames,I. Quasi-static anaysis of plane frames with initially curved members. São Paulo, EPUSP, 1989b. (Boletim Técnico do Departamento de Engenharia de Estruturas e Fundações, 8910).

105 PIMENTA, P. M. ; FRUCHTENGARTEN, J. Carga crítica de barras com consideração de empenamentos por torção - Uma formulação consistente. In: XV CILAMCE. Anais. 1994. v. 2, p. 1486-1495.

106 PIMENTA, P. M. ; MAZZILLI, C. E. N. Mínima correctio methodi inveniendi lineas curvas elasticii. São Paulo, EPUSP, 1986. (Boletim Técnico do Departamento de Engenharia de Estruturas e Fundações, 8613). 
107 PIMENTA, P. M. ; SOLER, J. G. M. Estabilidade de pórticos planos de concreto armado. In: SIMPÓSIO EPUSP SOBRE ESTRUTURAS DE CONCRETO, 1., São Paulo, 1989. Anais. v. 2, p. 501-527.

108 PIMENTA, P. M. ; YOJO, T. Análise geometricamente exata de pórticos espaciais através do método dos elementos finitos. In: XIII CILAMCE. Anais. 1992. v.1, p. 191-199.

109 PIMENTA, P. M. ; YOJO, T. Geometrically exact analysis of spacial frames with considerations of torsion warping. In: XIV CILAMCE. Anais. 1993. v.1, p. 21-30.

110 POWELL, G. H. Theory of nonlinear elastic structures. Journal of the structural division (ASCE). v. 95, n. St 12, p. 2687-2701, December, 1969.

111 PROENÇA, S. P. B. Notas sobre análise não-linear fisica de estruturas Parte 1: Teoria da plasticidade e técnicas numéricas. São Carlos, EESC, USP, 1989.

112 PROENÇA, S. P. B. Sobre modelos matemáticos do comportamento não-linear do concreto: análise crítica e contribuições. São Carlos, 1988. 330p. Tese (Doutorado) - Escola de Engenharia de São Carlos, Universidade de São Paulo.

113 PROENÇA, S. P. B. Noções Básicas de Reologia. São Carlos, EESC, USP, 1986

114 RACHID,M. ; MORI, D.D. Instabilidade: conceitos - aplicação na flambagem por flexão. São Carlos, EESC-USP, 1989.

115 RAJASEKARAN, S. ; MURRAY, D. W. Finite element solution of inelastic beam equation. Journal fo the structural division (ASCE). v.99, n. St 6, p. 1025-1041, June, 1973.

116 RAJASEKARAN, S. and MURRAY, D. W. Incremental finite element matrices. Journal fo the structural division (ASCE). v.99, n. St 12, p.2423-2438, December, 1973.

117 RAMAKRISNAN, V. ; BALAKRISHNAN, S. Elastic and limit state deformations of reinforced concrete space frames. The Indian Concrete Journal, v. 50, n. 3, p. 85-91, March, 1976.

118 RASHEED, H. A. S. ; DINNO, K. S. An efficient nonlinear analysis of RC sections. Computer \& Structures, v. 53, n. 3, p. 613-623, 1994. 
119 SCARLAT, A. S. Effect of soil deformability-related aspects of multistory buildings analysis. ACI Structural Journal, p. 156-162, MarchApril, 1993.

120 SCORDELIS, A. C. Computer models for nonlinear analysis of reinforced and prestressed concrete structures. ACI Journal, v. 29, n. 6, p. 116-135, Nov/Dez, 1984.

121 SILVA, R. M. Análise de estruturas tridimensionais de edificios altos com núcleos resistentes considerando o efeito P-A. São Carlos, 1989. 239p. Dissertação (Mestrado) - Escola de Engenharia de São Carlos, Universidade de São Paulo.

122 SOLER, J. G. M. Análise não-linear de pórticos espaciais de concreto armado. São Paulo, 1995. Tese (Doutorado) - Escola Politécnica, Universidade de São Paulo.

123 SOLER, J. G. M. ; PIMENTA, P. M. Estabilidade de pórticos planos de concreto armado. In: SIMPÓSIO EPUSP SOBRE ESTRUTURAS DE CONCRETO. EPUSP, São Paulo, p. 503-527, 1989.

124 SOUZA LIMA, V. M. ; VENÂNCIO FILHO, F. A noção de rigidez tangente no estudo de não-linearidade geométrica de estruturas reticuladas. In: CONGRESSO LATINO-AMERICANO SOBRE MÉTODOS COMPUTACIONAIS EM ENGENHARIA, 5., Salvador, 1984.

125 SOUZA LIMA, V. M. ; VENÂNCIO FILHO, F. Considerações sobre a não-linearidade geométrica em estruturas reticuladas. In: III ESCOLA DE MATEMÁTICA APLICADA, LCC-CNPQ, Rio de Janeiro, 1982.

126 SPILLERS, W. R. Geometric stiffness matrix for space frames. Computers \& Structures, v. 36, n. 1, p. 29-37, 1990.

127 SPRINGFIELD, J. ; ADAMS, P. F. Aspects of column design in tall steel buildings. Journal of the Structural Division (ASCE). v. 98. N. St 5, p.1069-1083, May, 1972.

128 TELLES, J. C. F. Análise do comportamento não-linear geométrico e fisico de pórticos planos de concreto armado. Rio de Janeiro, 1976. Dissertação (Mestrado) - COPPE, Universidade Federal do Rio de Janeiro. 
129 THOMPSON, J. M. T. ; HUNT, G. W. A general theory of elastic stability. London, John Wiley \& Sons Ltd, 1973.

130 TRANBERG, W. ; SWANNELL, P. ; MEEK, J. L. Frame collapso using tangent stiffness. Journal of the Structural Division (ASCE). v. 102, n.St 3, p. 659-675, 1976.

131 TURNER, M. J. ; DILL, E. H. ; MARGIN, H.C. ; MELOSH, R.J. Large deflection analysis of complex structures subjected to heating and external load. Journal of the Aerospace Sciences, v. 27, Feb., 1960.

132 TURNER, M.J. ; CLOUGH, R.W. ; MARTIN, H.C. ; TOPP, L.J. Stiffness and deflection analysis of complex structures. Journal of Aeronautical Sciences, v. 23, p. 805-823, 1956.

133 VASCONCELOS A. C. ; FRANCO, M. Practical assessment of second order effects in tall buildings. In: COLLOQUIUM ON THE CEB-FIP MODEL CODE 90, COPPE/CEB, Rio de Janeiro, Agosto, 1991.

134 VECCHIO, F. J. ; COLLINS, M. P. The modified compression-field theory for reinforced concrete elements subjected to shear. ACI Journal, p. 219-231, March-April, 1986.

135 WEN, R. K. ; RAHIMZADEH, J. Nonlinear elastic frame analysis by finite element. Journal of Structural Engineering (ASCE), v. 109, n.8, p.1952-1971, 1983.

136 WONG, M. B. ; TIN-LOI, F. Analysis of frames involving geometrical and material nonlinearities. Computers \& Structures, v. 34, n.4, p.641646, 1990.

137 WONG, M. B. ; TIN-LOI, F. Geometrically nonlinear analysis of elastic framed structures. Computers \& Structures, v. 34, n.4, p. 633-640, 1990.

138 WOOD, C. R. ; BEAULIEU, D. ; ADAMS, P. F. Column design by pdelta method. Journal of the Structural Division (ASCE), v. 102, n. St2, p. 411-427, February, 1976.

139 YAMADA, Y. ; YOSHIMURA, N. ; SAKURAI, T. Plastic stress-strain matrix and its applications for the solution of elastic-plastic problems of FEM. International Journal Mechanics Science, v. 10, p. 343-354, 1968. 
140 YANG, Y. B. ; KUO, S. R. Consistent frame buckling analysis by finite element method. Journal of Structural Engineering (ASCE), v. 117, n.4, p. 1053-1069, April, 1991.

141 YURA, J. A. The effective length of columns in umbraced frames. AISC Engineering Journal, V. 8, n. 2, p. 37-42, April, 1971

142 ZIEMIAN, R. D. ; Mc GUIRE, W. ; DEIERLEIN, G. G. Inelastic limit states design. Part I: Planar frame studies. Journal of Structural Engineering (ASCE), v. 118, n. 9, p. 2532-2549, September, 1992.

143 ZIENCKIEWICZ, O. C. The finite element method in engineering science. New York, McGraw Hill, 1971.

144 ZIENCKIEWICZ, O. C. ; CHEUNG, Y. K. The finite element method in structural and continuum mechanics. New York, McGraw Hill, 1967. 